\title{
Rotating Detonation Combustion: A Computational Study for Stationary Power Generation
}

\author{
Sergio Escobar
}

Follow this and additional works at: https://researchrepository.wvu.edu/etd

\section{Recommended Citation}

Escobar, Sergio, "Rotating Detonation Combustion: A Computational Study for Stationary Power Generation" (2014). Graduate Theses, Dissertations, and Problem Reports. 5549.

https://researchrepository.wvu.edu/etd/5549

This Dissertation is protected by copyright and/or related rights. It has been brought to you by the The Research Repository @ WVU with permission from the rights-holder(s). You are free to use this Dissertation in any way that is permitted by the copyright and related rights legislation that applies to your use. For other uses you must obtain permission from the rights-holder(s) directly, unless additional rights are indicated by a Creative Commons license in the record and/ or on the work itself. This Dissertation has been accepted for inclusion in WVU Graduate Theses, Dissertations, and Problem Reports collection by an authorized administrator of The Research Repository @ WVU.

For more information, please contact researchrepository@mail.wvu.edu. 


\title{
Rotating Detonation Combustion: A Computational Study for Stationary Power Generation
}

\author{
Sergio Escobar \\ Dissertation submitted \\ to the Benjamin M. Statler College of Engineering and Mineral Resources \\ at West Virginia University \\ in partial fulfillment of the requirements for the degree of \\ Doctor of Philosophy in \\ Mechanical Engineering \\ Ismail Celik, Ph.D., Chair \\ John M. Kuhlman, Ph.D. \\ V'yacheslav Akkerman, Ph.D. \\ Wade Huebsch, Ph.D. \\ Andrew Nix, Ph.D. \\ George A. Richards, Ph.D. \\ Donald H. Ferguson, Ph.D. \\ Department of Mechanical and Aerospace Engineering \\ Morgantown, West Virginia \\ 2014
}

Keywords: Rotating Detonation Combustion, Detonation, Computational Fluid Dynamics, Shock wave, Pressure Gain Combustion, CFD, Non Reflective Boundary Conditions, ANSYS Fluent. 


\section{ABSTRACT}

\section{Rotating Detonation Combustion: A Computational Study for Stationary Power Generation}

by Sergio Escobar

The increased availability of gaseous fossil fuels in The US has led to the substantial growth of stationary Gas Turbine (GT) usage for electrical power generation. In fact, from 2013 to 2104 , out of the 11 Tera Watts-hour per day produced from fossil fuels, approximately $27 \%$ was generated through the combustion of natural gas in stationary GT. The thermodynamic efficiency for simple-cycle GT has increased from $20 \%$ to $40 \%$ during the last six decades, mainly due to research and development in the fields of combustion science, material science and machine design. However, additional improvements have become more costly and more difficult to obtain as technology is further refined. An alternative to improve GT thermal efficiency is the implementation of a combustion regime leading to pressure-gain; rather than pressure loss across the combustor. One concept being considered for such purpose is Rotating Detonation Combustion (RDC). RDC refers to a combustion regime in which a detonation wave propagates continuously in the azimuthal direction of a cylindrical annular chamber. In RDC, the fuel and oxidizer, injected from separated streams, are mixed near the injection plane and are then consumed by the detonation front traveling inside the annular gap of the combustion chamber. The detonation products then expand in the azimuthal and axial direction away from the detonation front and exit through the combustion chamber outlet. 
In the present study Computational Fluid Dynamics (CFD) is used to predict the performance of Rotating Detonation Combustion (RDC) at operating conditions relevant to GT applications. As part of this study, a modeling strategy for RDC simulations was developed. The validation of the model was performed using benchmark cases with different levels of complexity. First, 2D simulations of non-reactive shock tube and detonation tubes were performed. The numerical predictions that were obtained using different modeling parameters were compared with analytical solutions in order to quantify the numerical error in the simulations. Additionally, experimental data from laboratory scale combustors was used to validate $2 \mathrm{D}$ and 3D numerical simulations. The effect of different modeling parameters on RDC predictions was also studied. The validated simulation strategy was then used to assess the performance of RDC for different combustion chamber geometries and operating conditions relevant to GT applications. As a result, the limiting conditions for which continuous detonation and pressure gain combustion can be achieved were predicted and the effect of operating conditions on flow structures and RDC performance was assessed.

The modeling strategy and the results from this study could be further used to design more efficient and more stable RDC systems. 


\section{Acknowledgements}

Receiving a doctoral degree would have not been possible, for me, without the support of Dr. Ismail Celik. I would like to thank him for his guidance and mentoring during my graduate school. He has taught me, through his actions, that persistence, hard work and discipline; combined with an analytical and critical mind are the most relevant requirements to be a successful scientist and engineer. I would like to express my appreciation to the dedication he provides to all of his students. It has been a privilege to have him as my research and academic advisor. I would also like to express my gratitude to the other members of my graduate committee: Dr. John Kuhlman, Dr. V'yacheslav Akkerman, Dr. Wade Huebsch, Dr. Andrew Nix, Dr. George A. Richards and Dr. Donald H. Ferguson. I hold in great value the advice and the time dedicated for the development of my PhD. Dissertation and my research work. I am also very grateful to the United States Department of Energy (USDOE) National Energy Laboratory and Technology (NETL) for funding my research. I would also like to thank the professors from the Benjamin Statler College of Engineering and Mineral Resources, and the Mechanical and Aerospace Engineering department. They have provided me with the knowledge necessary to tackle the challenges I will face in the future of my professional career.

As a member of the Computational Fluid Dynamics and Applied Multiphysics Center (CFD \& AMP), I was fortunate to find the support, guidance -but most importantly- the camaraderie from my fellow labmates. Therefore, I would like to thank the students and postdocs that were part of our group in the past; they helped me during the first stages of my graduate studies. Thanks to Dr. Ertan Karaismail, Dr. Fatma N. Cayan, Dr. Jagannath Nanduri, and Dr. Suryanarayana R. Pakalapati. Special thanks to Dr. Pakalapati for his patience and dedication while training me in the disciplines of CFD and computer programming. I also want to thank Dr. Steven Rowan, Dr. Tao Yang, Dr. Jose A. Escobar, Dr. Hairy Sezer, Gennaro Campitelli, Albio Gutierrez, Jerry Mason, Sharad Chand, Satish Guda, Engin Baran and Mehmet Aygun for the good times shared during the last few years.

I also want to express sincere gratitude to my family. Their unconditional support and love has always kept me motivated to move forward and to do my best to excel in everything I do. I will always be in debt for the sacrifices that my parents, Guillermo and Amanda, have made to provide me with the best opportunities in life. My father and my mother are my role models in dedication, perseverance and integrity. I would also like to thank my sister Claudia, despite the geographical distance, I have always known that you have my back no matter the circumstances. Last but not least, I also want to thank the love of my life, Laura Gil. She has been my companion during the most stressful times of my graduate school. It is thanks to her serenity, support and kind love that I was able to maintain calm and get the best out of every situation. 


\section{Contents}

Abstract

Acknowledgements

Contents iv

List of Figures

$\begin{array}{ll}\text { List of Tables } & \text { ix }\end{array}$

Acronyms

Symbols

1 Introduction 1

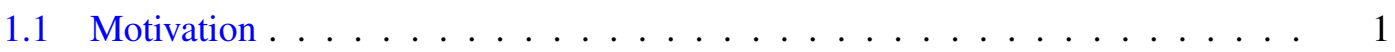

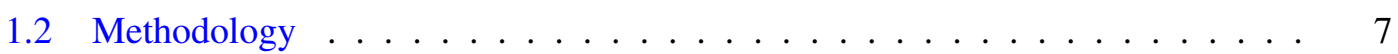

1.2.1 Model Validation . . . . . . . . . . . . . . . . . . 7

1.2.2 Sensitivity Analysis of the Modeling Strategy . . . . . . . . . . . . 7

1.3 Objectives ....................... 8

2 Literature Review 9

2.1 Detonation Phenomena . . . . . . . . . . . . . . . . . . . . 9

2.2 Thermodynamic Efficiency of Detonation Combustion in Power Cycles . . . . 14

2.3 Experimental Studies of Rotating Detonation Combustion . . . . . . . . . . . . 17

2.4 Numerical and Analytical Studies of Rotating Detonation Combustion . . . . . 21

2.5 Conclusions from Literature Review . . . . . . . . . . . . . . . . . 27

3 Model Considerations $\quad \mathbf{2 8}$

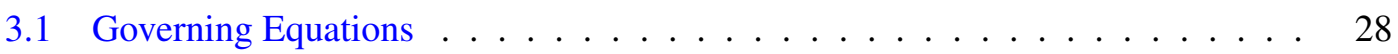

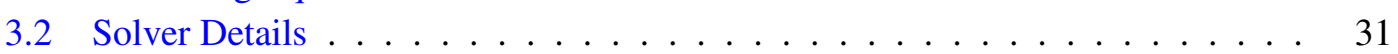

3.3 Computational Domain . . . . . . . . . . . . . . . . . . . 32

3.4 Boundary Conditions . . . . . . . . . . . . . . . . . 36

3.5 Initial Condition . . . . . . . . . . . . . . . . . . . . . . . 42

3.6 Conclusions from Model Considerations . . . . . . . . . . . . . . . . 45 
4 Model Validation $\quad 46$

4.1 Non Reactive Shock Tube Simulations . . . . . . . . . . . . . . . . . . . . . 46

4.1.1 Results using the Density Based Solver (DBS) . . . . . . . . . . . . . 48

4.1.2 Results using the Pressure Based Solver (PBS) . . . . . . . . . . . . 52

4.2 Detonation Tube Simulations . . . . . . . . . . . . . . . . . . . 55

4.2.1 Influence of chemical model on CJ and ZND predictions . . . . . . . 55

4.3 Simulation of Rotating Detonation Combustion for Model Validation . . . . . . 64

4.3.1 Two Dimensional Simulations of RDC for Model Validation . . . . . . 66

4.3.2 Three Dimensional Simulations of RDC for Model Validation . . . . . 75

4.4 Conclusions from Model Validation . . . . . . . . . . . . . . . . . . 92

5 Rotating Detonation Combustion at Elevated Pressures 96

5.1 Adaptation of 1D Model as a Predictive Tool . . . . . . . . . . . . . . . 96

5.2 Two Dimensional Simulations of RDC at Elevated Pressures . . . . . . . . . . 102

5.2.1 Application of Non Reflective Boundary Condition for RDC Simulations 102

5.3 Conclusions from Rotating Detonation Combustion at Elevated Pressures . . 124

6 General Conclusions $\quad 126$

6.1 Recommendation for Future Work . . . . . . . . . . . . . . . . . . . 128

A Chemical Model and Reaction Mechanisms $\quad 129$

B Inlet Boundary Condition - UDF 134

C Numerical Simulations of Non-Reactive Shock Tube Problem 138 


\section{List of Figures}

1.1 Schematic representation of Rotating Detonation Combustion . . . . . . . . . . 3

1.2 Temperature contours of RDC in a cylindrical chamber for stoichiometric $\mathrm{H} 2-$ Air mixture at a fgiven instant . . . . . . . . . . . . . . 3

1.3 RDC flow structures and discontinuities $\ldots \ldots \ldots \ldots$

2.1 Deflagration and Detonation front structure . . . . . . . . . . . . . . . 10

2.2 Chapman Jouguet state for $H_{2}+$ Air . . . . . . . . . . . . . . . . . 12

2.3 Detonation Length Scale . . . . . . . . . . . . . . . . . . . . . . . . . . . . . . 13

2.4 Brayton and Humphrey cycles . . . . . . . . . . . . . . . . . . . 15

2.5 Humphrey, FJ and ZND cycles for stoichiometric $H_{2}$-Air $\ldots \ldots \ldots$

2.6 NETL RDC experimental set up . . . . . . . . . . . . . . . . 20

2.7 Control volume schematic for analytical 1D RDC model . . . . . . . . . . . . . . . 22

2.8 2D Temperature contour from RDC simulations . . . . . . . . . . . . . 26

3.1 ANSYS Fluent DBS and PBS $\ldots \ldots \ldots \ldots \ldots \ldots \ldots \ldots$

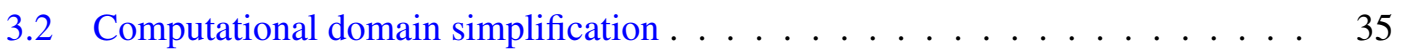

3.3 Computational Meshes . . . . . . . . . . . . . . . . . 36

3.4 Simple initiation strategy for 2D domain . . . . . . . . . . . . . . . 43

3.5 Initial condition for 2D RDC simulation from 1D model . . . . . . . . . . . 44

4.1 Schematic description of the non reactive shock tube problem . . . . . . . . . . 47

4.2 Pressure distribution for non reactive shock tube simulation DBSE-ROE-GC. . 49

4.3 Pressure distribution for non reactive shock tube simulation DBS-AUSM-GC-2U. 50

4.4 Local error distribution for non reactive shock tube predictions using DBS-

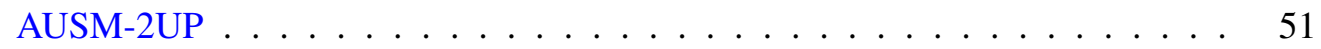

4.5 Spatial error $\ell^{2}$-norm for non reactive shock tube $\ldots \ldots \ldots . \ldots . \ldots 52$

4.6 Spatial error $\ell^{2}$-norm for non reactive shock tube . . . . . . . . . 53

4.7 2D CFD shocktube simulation results PBS-PISO-SQC-2U and 2nd Order in

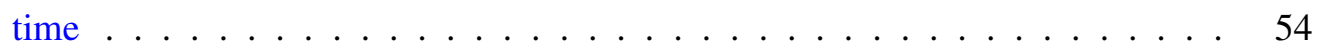

4.8 ZND prediction for stoichiometric $\mathrm{H}_{2}$-air with single step and multistep reaction models . . . . . . . . . . . . . . . . . . 57

4.9 Schematic for the detonation tube problem . . . . . . . . . 58

4.10 Planar detonation wave CFD prediction for 1 step reaction mechanism and $\delta x=$

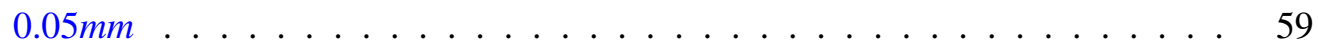

4.11 2D detonation wave CFD prediction for 1 step reaction mechanism, region near the detonation front . . . . . . . . . . . . . . . . . 60

4.12 2D detonation wave CFD prediction for multi step reaction mechanism and $\delta x=$

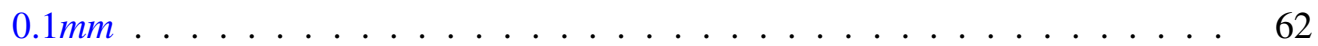


4.13 2D detonation wave CFD prediction for multi step reaction mechanism, region near the detonation front . . . . . . . . . . . . . . . .

4.14 2D CFD shocktube simulation results PBS-PISO-SQC-2U and 2nd Order in

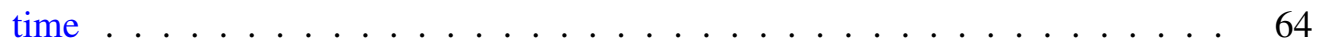

4.15 Schematic representation of combustor geometry for model validation . . . . 65

4.16 2D temperature contours for PBS-PISO-AUSM-2UP RDC prediction . . . . . 67

4.17 2D density gradient contour for PBS-PISO-SQC-2UP RDC prediction . . . . . 68

4.18 2D density gradient contour with stream line locations . . . . . . . . . . . 69

4.19 Comparison of pressure history between 2D CFD RDC simulation PBS with 1 step reaction model and experiments . . . . . . . . . . . . . . . . . 69

4.20 2D temperature contour for DBS-AUSM-SQC-2UP and multi step chemistry RDC prediction . . . . . . . . . . . . . . . . . 70

4.21 2D density gradient contour for DBS and multi step chemistry . . . . . . . . 71

4.22 Comparison of pressure history between 2D CFD RDC simulation with the DBS and multi step reaction mechanism and experiments . . . . . . . . . . 72

$4.232 \mathrm{D}$ contour for major species and pollutant concentrations in RDC . . . . . . . 73

4.24 Two dimensional contours of RDC simulation with multi step reaction mechanism and $\Phi=0.5 \ldots \ldots \ldots \ldots$. . . . . . . . . . . . . . . . 74

4.25 3D temperature and pressure contour for 1 step and multi step chemistry model $\quad 76$

4.26 Cross sectional pressure and temperature contours from 3D simulation _ . . . 77

4.27 Comparison of pressure history between 3D CFD RDC simulation and experi-

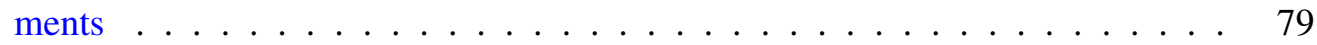

4.28 Temperature and pressure gradient contours for 3D RDC with $D_{\text {in }}=95 \mathrm{~mm} \quad \ldots \quad 81$

4.29 Cross sectional pressure and temperature contours from 3D inviscid simulations for RDC chamber with $D_{i n}=80 \mathrm{~mm} \ldots \ldots \ldots \ldots \ldots$

4.30 Radial distribution of temperature and pressure for RDC camber with $D_{\text {in }}=$ $95 \mathrm{~mm}$ and $80 \mathrm{~mm} \ldots \ldots \ldots \ldots \ldots \ldots \ldots$

4.31 3D temperature contour and reaction front surface for 3D laminar and adiabatic

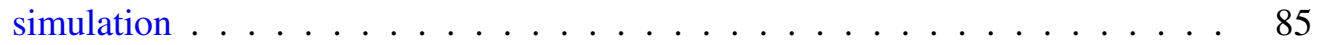

4.32 Temperature and heat flux contours for thin wall simulation of RDC . . . . . 86

4.33 Local temperature and normalized heat flux history for thin wall simulation of RDC . . . . . . . . . . . . . . . . .

4.34 Temperature and heat flux contours for 3D simulation of RDC including outer wall solid region . . . . . . . . . . . . . . . . . .

4.35 Local temperature and normalized heat flux history for thin wall simulation of RDC . . . . . . . . . . . . . . . . . .

4.36 Temperature, pressure and reaction front contour plots for 3D RDC with injec-

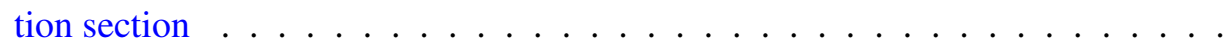

4.37 Cross sectional axial velocity contour from 3D simulation for RDC chamber with injection geometry . . . . . . . . . . . . . . . 92

4.38 Pressure history for 3D RDC including injection section $\ldots \ldots$. . . . . . . 93

5.1 Comparison of 2D analytical model prediction with 2D CFD results . . . . . 98

5.2 2D Analytical model prediction of static pressure for RDC at elevated pressures $\quad 99$

5.3 Analytical model prediction of deflagration front shape for RDC at elevated pressures and various injection geometries . . . . . . . . . . . . 100

5.4 Analytical model prediction of $\Theta$, influence of $P_{\infty}$ and $A_{t} / A_{e} \ldots \ldots \ldots 101$

5.5 Analytical model prediction of losses in total pressure in RDC . . . . . . . . . 101 
5.6 Outlet pressure prediction with $\sigma=0.15$, ill-imposed problem . . . . . . . . 102

5.7 Theoretical reflection coefficient for the NRBC with $\sigma=-2 \pi \ldots \ldots \ldots$

5.8 Outlet pressure prediction with $\sigma=-2 \pi$, well-imposed problem . . . . . . . . 104

5.9 Instantaneous distribution of reflection coefficient for 2D RDC simulation with

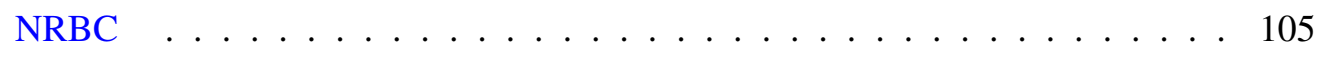

5.10 Temperature contours for 2D simulations of RDC with $A t / A e=0.08$ and $P_{\infty}=$ $0.11,1.0$ and $2.0 \mathrm{~atm} \ldots \ldots \ldots \ldots$. . . . . . . . . . . . . . . . . . . . .

5.11 Density gradient contours for $2 \mathrm{D}$ simulations of RDC with $A t / A e=0.08$ and $P_{\infty}=0.11,1.0$ and $2.0 \mathrm{~atm} \ldots \ldots \ldots \ldots \ldots$

5.12 Mach number contours for 2D simulations of RDC with At/Ae $=0.08$ and $P_{\infty}=0.11,1.0$ and $2.0 \mathrm{~atm} \ldots \ldots \ldots \ldots \ldots$

5.13 Mach number and stagnation pressure distribution at the combustion chamber outlet for $A t / A e=0.08$ and $P_{\infty}=0.11,1.0$ and $2.0 \mathrm{~atm} \ldots \ldots 110$

5.14 Prediction of deflagration front shape from $2 \mathrm{D}$ RDC simulation with $A_{t} / A_{e}=$ 0.08 at elevated pressures . . . . . . . . . . . . . . . . . 111

5.15 Temperature contours for 2D simulations of RDC with $A t / A e=0.2$ and $P_{\infty}=$ $2.0,4.0$, and $6.0 \mathrm{~atm} \ldots \ldots \ldots \ldots \ldots \ldots$

5.16 Density gradient contour plots for 2D simulations of RDC with $A t / A e=0.2$ and $P_{\infty}=2.0,4.0$, and $6.0 \mathrm{~atm} \ldots \ldots \ldots \ldots$

5.17 Mach number contour plots for 2D simulations of RDC with $A t / A e=0.2$ and $P_{\infty}=2.0,4.0$, and $6.0 \mathrm{~atm} \ldots \ldots \ldots \ldots \ldots$. . . . . . . . . 114

5.18 Prediction of deflagration front shape from $2 \mathrm{D}$ RDC simulation with $A_{t} / A_{e}=$ 0.2 at elevated pressures . . . . . . . . . . . . . . . 115

5.19 Temperature contour plots for 2D simulations of RDC with At/Ae $=0.4$ and $P_{\infty}=6.0,8.0$, and $8.5 \mathrm{~atm} \ldots \ldots \ldots \ldots \ldots \ldots \ldots \ldots$
Density gradient contour plots for $2 \mathrm{D}$ simulations of RDC with $A t / A e=0.4$ and

5.20 Density gradient contour plots for $2 \mathrm{D}$ simulations of RDC with $A t / A e=0.4$ and
$P_{\infty}=6.0,8.0$ and $8.5 \mathrm{~atm} \ldots \ldots \ldots \ldots \ldots \ldots \ldots \ldots \ldots \ldots \ldots \ldots \ldots$

5.21 Mach number contour plots for 2D simulations of RDC with At/Ae $=0.4$ and $P_{\infty}=6.0,8.0$, and $8.5 \mathrm{~atm} \ldots \ldots \ldots \ldots \ldots$

5.22 Prediction of detonation wave velocity from $2 \mathrm{D}$ RDC simulation with $A_{t} / A_{e}=$ $0.08,0.02$ and 0.4 at elevated pressures . . . . . . . . . . . . . 120

5.23 Prediction of mass flux at the outlet of the combustion chamber from 2D RDC simulation with $A_{t} / A_{e}=0.08,0.02$ and 0.4 at elevated pressures . . . . . . 120

5.24 Prediction of total pressure losses in the the combustion chamber from 2D RDC simulation with $A_{t} / A_{e}=0.08,0.02$ and 0.4 at elevated pressures . . . . . . 121

5.25 Prediction of geometric factor $\Theta$ from $2 \mathrm{D}$ RDC simulation with $A_{t} / A_{e}=0.08,0.02$ and 0.4 at elevated pressures . . . . . . . . . . . . . . . 122

5.26 Comparison of predicted RDC performance parameters from analytical model and 2D CFD calculations . . . . . . . . . . . . . . . . . . . 123 


\section{List of Tables}

2.1 Deflagration and Detonation combustion comparison [Kuo, 1986] . . . . . . 11

3.1 Geometric parameters for $\mathrm{RDC}$ in annular chamber . . . . . . . . . . . . 33

3.2 Initial conditions for $2 \mathrm{D}$ RDC simulations . . . . . . . . . . . . . . . 43

4.1 Mesh and time step characteristics for non reactive shock tube problem . . . . 48

4.2 Chapman-Jouguet Prediction from the different chemical modeling strategies for $\mathrm{H}_{2}$-air and syngas-air mixtures $\ldots \ldots \ldots \ldots \ldots 6$

4.3 ZND Prediction for $\mathrm{H}_{2}$-Air using the different chemical modeling strategies . . 56

4.4 CFD simulation of detonation wave prediction in a $2 \mathrm{D}$ channel, results using PBS-PISO-SQC-2UP and multiple mesh sizes. . . . . . . . . . . . . . . 61

4.5 CFD simulation of detonation wave prediction in a 2D channel, results for DBSAUSM-SQC-2UP, multiple mesh sizes and two chemical models. . . . . . . . . 61

4.6 Summary of detonation wave velocity prediction for model validation . . . . . 79

4.7 Influence of viscous terms on detonation wave velocity prediction for 3D simulations with PBS-PISO-SQC-2UP and 1 Step reaction mechanism . . . . . . 89

5.1 2D RDC Results for elevated outlet pressures . . . . . . . . . . . . . . . . 124

C.1 Numerical Simulations for Non Reactive Shocktube using DBS and Mesh-1 . . 138

C.2 Influence of mesh cell size on numerical Simulations for Non Reactive Shocktube. Calculated using DBS-AUSM-SQC-2U . . . . . . . . . . . . . 138

C.3 Numerical Simulations for Non Reactive Shocktube using PBS and Mesh-1 . . 139

C.4 Influence of mesh cell size on numerical Simulations for Non Reactive Shocktube. Calculated using PBS-CP-SQC-2U . . . . . . . . . . . . . . . . . . 139 


\section{Acronyms}

$1 U P$ 1st-Order Upwind

2UP 2nd-Order Upwind

3M 3rd-Order MUSCL

AUSM Advectio Upstream Splitting Method

AMR Adaptive-Mesh-Refinement

CJ Chapman-Jouguet

CFD Computational Fluid Dynamics

CFL Courant-Friederich-Lewis

DBS Density Based Solver

GC Green-Gaus Cell-based

GT Gas Turbine

LRM Linear Relaxation Method

MUSCL Monotonic Upstream Centered Scheme

MS Multi-Step Chemistry

NETL National Energy and Technology Laboratory

NS Navier Stokes

NRBC Non-Reflective Boundary Condition

PBS Pressure Based Solver

PDE Pulse Detonation Engine

PGC Pressure Gain Combstion

PISO Pressure-Implicit with Splitting of Operators

QUICK Quadratic Upstream Interpolation for Convective Kinematics

RDC Rotating Detonation Combustion

ROE ROE Flux-Difference Spliting Scheme

SQC Least- SQuare Cell-based 
UDF User Defined Function

URANS Unsteady Reynolds A veraged Navier Stokes

USDOE United States Department of Energy

WRE Wave Rotor Engine 


\section{Symbols}

\begin{tabular}{|c|c|c|}
\hline Symbol & Name & Unit \\
\hline$A_{e}$ & Cross sectional area at expansion plane of injection section & $m^{2}$ \\
\hline$A_{r}$ & Preexponential Factor & - \\
\hline$A_{t}$ & Throat Area & $m^{2}$ \\
\hline$C p_{\text {mix }}$ & Mean Specific Heat for constant pressure & $J / k g-K$ \\
\hline$\left[C_{k}\right]$ & Molar Concentration of species $\mathrm{k}$ & kmoles $/ \mathrm{m}^{3}$ \\
\hline$\left[C_{k, r}\right]$ & Molar Concentration of species $\mathrm{k}$ in reaction $\mathrm{r}$ & kmoles $/ \mathrm{m}^{3}$ \\
\hline$D_{i n}$ & Inner Diameter & $\mathrm{m}$ \\
\hline$D_{k}$ & Mass Diffusion Coefficient for species $\mathrm{k}$ & $m^{2} / s$ \\
\hline$D_{\text {mean }}$ & Mean Diameter & $\mathrm{m}$ \\
\hline$D_{\text {out }}$ & Outer Diameter & $\mathrm{m}$ \\
\hline E & Total Energy & $\mathrm{J}$ \\
\hline$E_{r}$ & Activation Energy & $\mathrm{J}$ \\
\hline$h_{\text {conv }}$ & Convection Coefficient & $W / m^{2}$ \\
\hline$h_{k}$ & Specific Enthalpy of species $\mathrm{k}$ & $J / k g$ \\
\hline$k_{b}^{r}$ & Backwards reaction rate for reaction $r$ & $1 / \mathrm{s}$ \\
\hline$k_{f}^{r}$ & Forward reaction rate for reaction $\mathrm{r}$ & $1 / \mathrm{s}$ \\
\hline$\ell^{2}$ & L-2 norm of a vector & - \\
\hline$L_{c}$ & Chamber's Length & $\mathrm{m}$ \\
\hline$\dot{m}_{\text {inlet }}$ & Mass flow rate at inlet & $\mathrm{kg} / \mathrm{s}$ \\
\hline$\dot{m}_{\text {inlet }}^{*}$ & Mass flow rate at inlet for chocked condition & $\mathrm{kg} / \mathrm{s}$ \\
\hline$M w_{k}$ & Molecular mass of species $\mathrm{k}$ & $\mathrm{kg} / \mathrm{kmole}$ \\
\hline$M w_{\text {mix }}$ & Mean Molecular mass of of the mixture & $\mathrm{kg} / \mathrm{kmole}$ \\
\hline$\vec{n}_{\text {wall }}$ & Normal vector to wall boundary & - \\
\hline$N_{k}$ & Number of reactions & - \\
\hline
\end{tabular}




\begin{tabular}{|c|c|c|}
\hline$N_{s}$ & Number of species & - \\
\hline$\hat{P}$ & Averaged pressure & atm \\
\hline$P^{\prime \prime}$ & Subsonic isentropic expansion static pressure & atm \\
\hline$P^{\prime}$ & Supersonic isentropic expansion static pressure & atm \\
\hline$P_{1}$ & Static Pressure in driven section & atm \\
\hline$P_{2}$ & Static Pressure in driver section & atm \\
\hline$P_{\infty}$ & Exhaust Pressure & atm \\
\hline$P_{C J}$ & Chapman-Jouguet Pressure & atm \\
\hline$P_{\text {exp }}$ & Static pressure at expansion plane & atm \\
\hline$P_{\text {inlet }}$ & Static pressure at expansion plane & atm \\
\hline$P_{o, m}$ & Stagnation pressure at the injection manifold & atm \\
\hline$q^{\prime \prime}$ & Heat flux & $W / m^{2}$ \\
\hline$R$ & Surrogate molecule for reactants mixture & - \\
\hline$\hat{R}$ & Gas Constant & $\mathrm{J} / \mathrm{kg-K}$ \\
\hline $\bar{R}$ & Universal Gas Constant & $\mathrm{J} / \mathrm{kmol}-\mathrm{K}$ \\
\hline$R_{f}$ & Reflection coefficient & \\
\hline$S_{L}$ & Laminar Flame Speed & $\mathrm{m} / \mathrm{s}$ \\
\hline$t$ & Time & $\mathrm{s}$ \\
\hline$T_{C J}$ & Chapman-Jouguet Temperature & $\mathrm{K}$ \\
\hline$T_{\text {exp }}$ & Static Temperature at expansion plane & $\mathrm{K}$ \\
\hline$T_{f, a d}$ & Adiabatic Flame Temperature & $\mathrm{K}$ \\
\hline$T_{o, m}$ & Stagnation Temperature at injection manifold & $\mathrm{K}$ \\
\hline$u_{x}$ & x Velocity Component & $\mathrm{m} / \mathrm{s}$ \\
\hline$u_{y}$ & y Velocity Component & $\mathrm{m} / \mathrm{s}$ \\
\hline$u_{z}$ & z Velocity Component & $\mathrm{m} / \mathrm{s}$ \\
\hline$U_{C J}$ & Chapman-Jouguet Velocity & $\mathrm{m} / \mathrm{s}$ \\
\hline$U_{D W}$ & Detonation Wave Velocity & $\mathrm{m} / \mathrm{s}$ \\
\hline$U_{\max }$ & Maximum Injection Velocity & $\mathrm{m} / \mathrm{s}$ \\
\hline$U_{p}$ & Particle Velocity & $\mathrm{m} / \mathrm{s}$ \\
\hline$U_{S W}$ & Shock Wave Velocity & $\mathrm{m} / \mathrm{s}$ \\
\hline$\vec{v}$ & Velocity vector & $\mathrm{m} / \mathrm{s}$ \\
\hline$\vec{v}_{\text {inlet }}$ & Velocity vector at inlet boundary & $\mathrm{m} / \mathrm{s}$ \\
\hline$\vec{x}$ & Position vector & $\mathrm{m}$ \\
\hline
\end{tabular}


$\begin{array}{ll}Y_{k} & \text { Mass fraction of species } \mathrm{k} \\ \left(Y_{k, \text { inlet }}\right) & \text { Mass fraction of species } \mathrm{k} \text { at inlet }\end{array}$

$\beta \quad$ Injection factor

$\delta t \quad$ Time step size in $\mathrm{x}$ direction $\mathrm{s}$

$\delta x \quad$ Computational cell size in $\mathrm{x}$ direction $\mathrm{m}$

$\delta x_{\min } \quad$ Minimum Computational cell size in $\mathrm{x}$ direction $\mathrm{m}$

Sy Computational cell size in y direction $\mathrm{m}$

$\Delta_{\text {air }} \quad$ Air injection annular gap $\mathrm{m}$

$\Delta_{c} \quad$ Chamber annular gap $\mathrm{m}$

$\Delta_{D W} \quad$ Detonation front height $\mathrm{m}$

$\Delta_{I} \quad$ Induction length $\quad \mathrm{m}$

$\Delta_{R} \quad$ Reaction length $\quad \mathrm{m}$

$\eta_{k, r}^{\prime} \quad$ Forward rate exponent for species $\mathrm{k}$ in reaction $\mathrm{r}$

$\eta_{k, r} \quad$ Backward rate exponent for species $\mathrm{k}$ in reaction $\mathrm{r}$

$\eta_{B} \quad$ Thermodynamic efficiency Brayton cycle

$\eta_{H} \quad$ Thermodynamic efficiency Humphrey cycle

$\gamma \quad$ Ratio of specific heats

$\mathcal{L}_{i} \quad$ Amplitude for characteristic wave i m

$\dot{\omega}_{k} \quad$ Production rate of species k $\mathrm{kg} / \mathrm{m}^{3}-\mathrm{s}$

$\Phi \quad$ Equivalence Ratio

$v_{k, r} \quad$ Stoichiometric coefficient for reactant $k$ in reaction $r$

$v_{k, r} \quad$ Stoichiometric coefficient for product $k$ in reaction $r$

$\rho \quad$ Density $\mathrm{kg} / \mathrm{m}^{3}$

$\rho_{o, m} \quad$ Density at stagnation condition in injection manifold $\mathrm{kg} / \mathrm{m}^{3}$

$\rho_{\text {inlet }} \quad$ Density at inlet boundary $\mathrm{kg} / \mathrm{m}^{3}$

$\sigma \quad$ linear relaxation coefficient for NRBC -

$\dot{\sigma} \quad$ Thermicity $1 / s$

$\tau_{i j} \quad$ Stress Tensor

$\tau_{I} \quad$ Induction Time $\quad \mathrm{s}$

$\tau_{R} \quad$ Reaction Time $\mathrm{s}$

$\theta_{R} \quad$ Section of the injection surface in which $\vec{v}_{i}$ nlet $>o \quad m$

$\Theta \quad$ Non dimensional geometric factor for the shape of the deflagration front - 
Dedicated to my father, Guillermo Escobar and my mother, Amanda Orrego. Thanks for your unconditional love and support. 


\section{Chapter 1}

\section{Introduction}

\subsection{Motivation}

During 2013-2014, 67\% of the electrical power in The US was generated from fossil fuel sources [EIA, 2013]; from which an average $27 \%$ of the 11 Tera Watts-hour per day originated through the combustion of natural gas using stationary power generation gas turbines (GT). The aforementioned scenario, along with the increasing availability of gaseous fuels (shale gas, and synthetic gas from coal), provide great motivation for the search of new strategies that increase the efficiency of stationary power generation GT.

During the last six decades, remarkable improvements were developed in the field of gas turbines. The efficiency for simple cycle gas turbines was improved from $20 \%$ in the early 1950 's up to more than $40 \%$ for modern GT. Additionally, thermal efficiency of up to $60 \%$ have been achieved due to the introduction of the combined power cycles [Saravanamuttoo et al., 2001]. Despite the circumstances; however, additional improvements are proven more costly and more difficult to obtain as technology is further refined.

Beginning in the 1950s, intermittent interest has been given to modifications on the gas turbine power cycle in order to replace the process of constant pressure heat addition by one through which some pressure gain is obtained. At present, gas turbine technology makes use of turbulent deflagration which occurs at relatively constant pressure. In such a process, the potential chemical energy present in the fuel-oxidizer mixture is transformed into heat and added to the working fluid, whilst the expansion of the combustion products does not perform any work towards the power cycle. As defined by [Richards et al., 1993], pressure-gain-combustion (PGC) is achieved when the mean total pressure at the combustor outlet is greater than at the 
inlet plenum.

Different constructs have been proposed through the last 40 years to implement combustion regimes leading to PGC into GT cycles. Early inventions involving complex coupling of piston-turbo machinery systems were proven to be impractical for industrial applications. Attempts to take advantage of the constant volume combustion behavior of the detonation phenomena were performed by Holzwart in the early 1930's [Hans, 1934]. Since then, methods such as pulsating combustion [Gemmen et al., 1995], pulse detonation engines (PDE) [Heiser and Pratt, 2002] and wave rotor engines (WRE) [Akbari et al., 2006] have been proposed as strategies to use pressure gain combustion for stationary gas turbine applications.

Although great advancements in all the previous concepts have been achieved, several technical difficulties are still to be solved before major industrial applications. Problems such as the need of intermittent source of ignition and constant transition from deflagration to detonation provide methods such as PDE and WRE with inherent difficulties for application on power generation gas turbines. As an alternate solution, the concept of rotating detonation combustion (RDC) has emerged during the last two decades. Although initial explorations focused on the viability of its application for propulsive purposes, recent research evidences interest in incorporating this regime for gas turbine applications.

Rotating detonation combustion (RDC) can be defined as the combustion regime under which a detonation wave propagates continuously in the azimuthal direction of a cylindrical annular chamber. In this regimen, the reactants are supplied in a bulk axial direction at the inlet plenum of the combustion chamber. The fresh reactants are injected from a premixture reservoir or from separate streams, as illustrated in Figure 1.1. In the latter, the fuel and oxidizer mix near the injection plenum. The detonation products expand behind the detonation wave in the azimuthal and axial direction exiting through the combustion chamber outlet. The unidirectional initiation of the detonation wave is obtained using an external initiation chamber. The initiation tube is placed in a tangential fashion to the annular chamber and filled with a highly reactive mixture, such as $\mathrm{H}_{2}-\mathrm{O}_{2}$ or $\mathrm{C}_{2} \mathrm{H}_{2}-\mathrm{O}_{2}$. On the end of the initiation tube opposite to the annular chamber, an energy discharge in the form of a spark ignites the reactive mixture. The spark generates a deflagration front that accelerates inside the initiation tube and transitions into a detonation wave. The detonation wave enters the annular chamber with a preferable direction, and if the conditions are appropriate, continuous detonation is achieved.

A characteristic temperature distribution is formed inside RDC annular chambers, such distribution is illustrated in Figure 1.2. This contours plot was taken from a three dimensional 


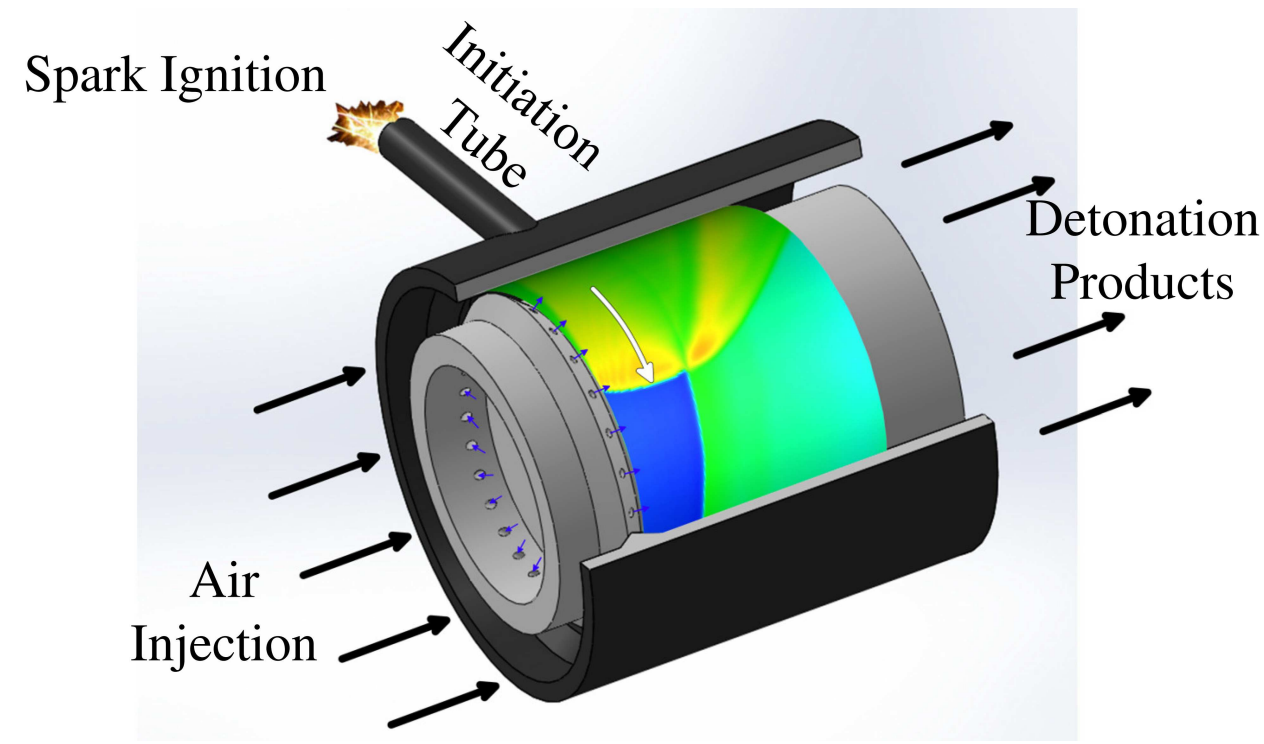

FIGURE 1.1: Schematic representation of Rotating Detonation Combustion

simulation of $\mathrm{H}_{2}$-Air RDC performed as part of the present study. Further details of these results will be provided in the following sections of this document.

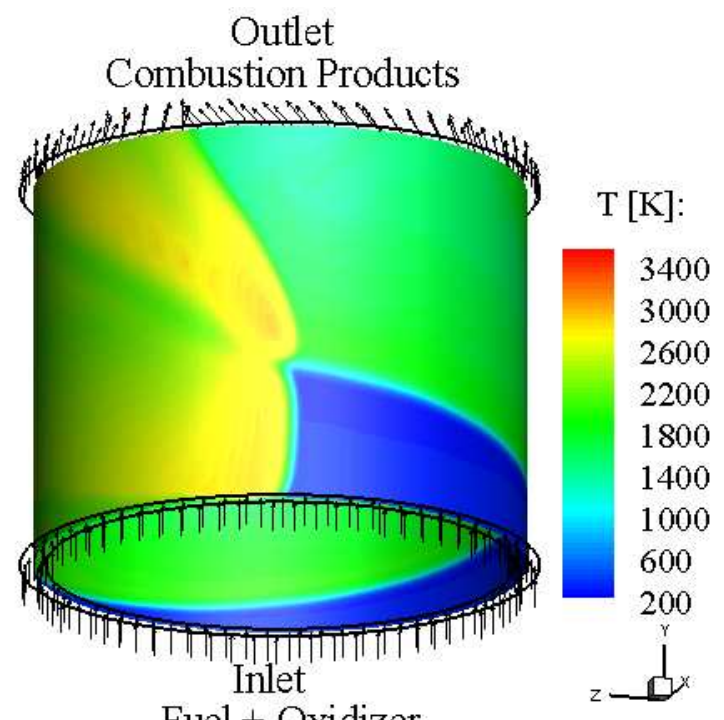

FIGURE 1.2: Temperature contours of RDC in a cylindrical chamber for stoichiometric H2-Air mixture at a given instant

The concept of RDC, also referred to as continuous detonation wave combustion, was first demonstrated during the late 1950's by Voitsekhovsky [1960]. It has been shown that RDC has several advantages over other detonation concepts for gas turbine applications. When compared with PDE, RDC presents several interesting features. These include, for instance, requiring a unique initiation event since the continuity of the detonation wave is guaranteed by the continual feeding of the reactants and unidirectionality of its propagation. Additionally, higher 


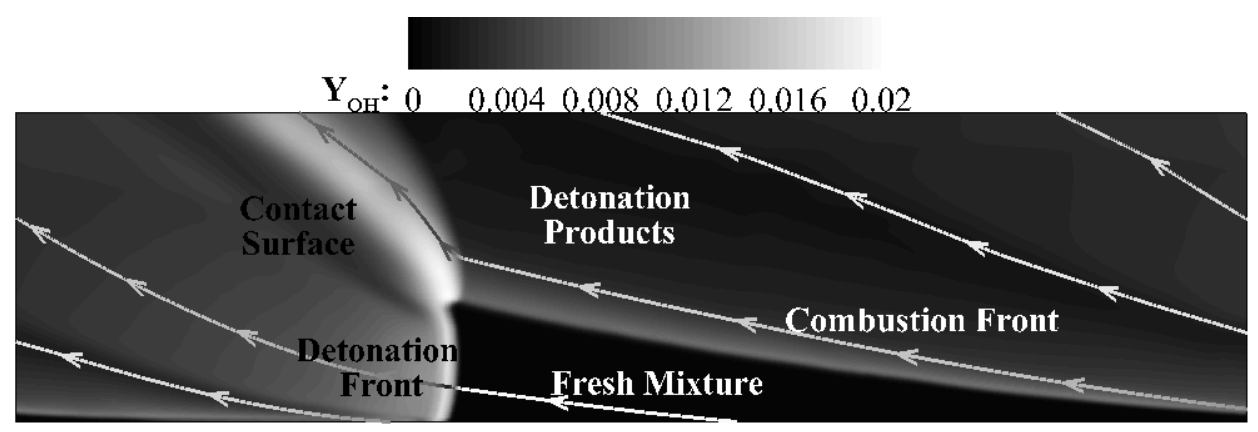

(a)

FIGURE 1.3: RDC flow structures and discontinuities, $\mathrm{OH}$ mass fraction $Y_{O H}$ contour from 2D CFD [Escobar et al., 2013].

detonation wave frequencies can be achieved $(\approx 5 \mathrm{kHz}$ Liu et al. [2012]). In contrast to PDE, $\mathrm{RDC}$ operation requires a unique initiation event. Therefore, transition from deflagration to detonation is not as critical phenomenon for RDC as it is for PDE. In addition, higher frequency leads to a more uniform flow at the combustor outlet, when compared to a single-tube PDE.

As first noticed by Voitsekhovsky [1960] distinctive flow structures arise inside the combustion chamber during continuous detonation regime. The interaction of the detonation wave with the injected mixture and the detonation products generate a complex group of interconnected flow structures. The results in Figure 1.2 and Figure 1.3 were obtained as part of the current study. The high $Y_{O H}$ region in Figure 1.3 indicate the main discontinuities present in RDC flows.

The edge at the bottom of the image corresponds to the inlet plenum, while the horizontal edge at the top corresponds to the outlet of the combustion chamber. The dark region labeled as fresh mixture in Figure 1.3(b) refers to the non-reacted mixture of fuel and oxidizer ahead of the detonation wave. The injection velocity, mass flux and pressure of the reactants depend on the pressure distribution inside the combustion chamber. A pressure distribution is formed due to the presence of the detonation wave, therefore the static pressure inside the combustion chamber is a function of location and time. The injection velocity of the reactants is an important factor that determines the possibility of continuous detonation. If the injection conditions do not lead to a layer long enough to sustain the detonation wave, continuous detonation operation cannot be achieved. The details on the behavior of the injection region and its implications on RDC performance are covered as part of the present study. On the left of the fresh mixture region, it is possible to identify the detonation front; characterized as a high $\mathrm{OH}$ concentration region. The interaction of the detonation wave with the combustion products from the previous cycle forms an oblique shock wave that extends from the end of the detonation front to the outlet of the combustion chamber. The contact of injected mixture with detonation products at elevated temperature creates a combustion front that limits the fresh mixture region in its characteristic 
wedge shape. The presence of this combustion front has negative repercussion on the overall efficiency of RDC operation. Further details of the flow structures will be provided in the literature review section.

During the last two decades the field of computational fluid dynamics (CFD) has contributed to a better understanding of RDC. However, several difficulties are present in the numerical study of RDC. The compressible nature of the flow imposes restrictions on the solution strategy to be used. Since shock waves, detonation waves and flame fronts are the main structures governing RDC performance, flow discontinuities should be adequately predicted by the selected spatial discretization methods. Additionally, stringent restrictions in spatial and temporal partitioning of the computational domain are imposed due to the high velocities of the expanding detonation products; as well as by wave velocities higher than $1000^{m} / \mathrm{s}$. The reactive nature of the flow requires appropriate handling of species transport equations and their rate of formation and consumption due to chemical reactions. The selection of proper reaction mechanisms is necessary to achieve a balance between computational cost and prediction detail without jeopardizing accurate prediction of variables of interest such as pollutant concentrations $\left(\mathrm{CO}, \mathrm{CO}_{2}\right.$ and $\left.\mathrm{NO}_{\mathrm{x}}\right)$, among others. 
In addition to the complex nature of the flow in RDC, modeling restrictions also arise due to operating conditions and geometrical configurations of the combustion chambers. Injection systems for rotating detonation combustion consist of convergent divergent nozzles, annular slots and/or injection orifices that vary greatly in scale and often have three dimensional implications on the flow. For the benefit of simplicity and computational cost, it is a commonly used practice [Hishida et al., 2009, Kindracki et al., 2011b, Zhdan et al., 1990] to exclude the injection geometry from the computational domain and to incorporate its physics through an inlet boundary condition model. Zhdan first proposed a model that calculates the state of the injection gases (premixed fuel and oxidizer) assuming the ideal behavior of convergent divergent Laval micro nozzle with variable back pressures [Zhdan et al., 1990]. Further insight of this model will be provided in following sections. Careful treatment of the outlet condition is also required. The possibility of reflected pressure waves from the outlet boundary influencing the detonation front behavior should be eliminated or minimized. The inappropriate treatment of outlet conditions may lead to inaccurate prediction of the detonation wave front dynamics.

Along with the difficulties present in the numerical modeling of RDC, there is also insufficient experimental data for the characterization of RDC, especially at elevated outlet pressures. This can be related to the fact that the main interest in RDC has been in its application for propulsive systems. Therefore, experimental and numerical studies have focused on the behavior of RDC under conditions and geometric configurations pertinent to these applications. The interest on propulsive application has led to the characterization of mostly relatively small combustion chambers, with mean diameters of $\approx 100 \mathrm{~mm}$. Additionally, in most studies, mass flow rates and equivalence ratios have been selected to obtain stable operation and maximize specific impulse. Further more, the majority of experimental and numerical research of RDC has been performed for low outlet pressure conditions, leading to critical or near critical exhaust regime of the detonation products. However, as presented by [Schwer and Kailasanath, 2011], changes occur in RDC performance as the exhaust pressure is increased, as the variation in the outlet mass flow rate and on the velocity of the detonation products.

Initial explorations of RDC operation under increased outlet pressure has been performed by [Schwer and Kailasanath, 2011]. However, exhaust pressures relevant for gas turbine operation need further examination. Operational conditions for gas turbine application under which continuous detonation can be achieved have not yet been reported. Such study would present a useful contribution towards the assessment of the viability of the incorporation of RDC for power generation. Predictions of thermodynamic efficiency of RDC under gas turbine operation conditions would help determine whether the application of this combustion regime leads to possible improvements over the current gas turbine technology. 


\subsection{Methodology}

In the present work computational fluid dynamics (CFD) is used to evaluate the performance of RDC under operating conditions relevant to gas turbine applications with the goal to determine how efficiency improvement in the gas turbine power generation cycle can be achieved. The proposed methodology is the optimization of a computational strategy for RDC simulation, based on the principles of low computational cost while maintaining the ability to capture the main features of RDC relevant to gas turbine operations.

A systematic approach to the development of the modeling strategy for rotating detonation simulations is deemed necessary due to the complex nature of the flow formed during continuous detonation combustion. The development of the computational strategy is achieved by the use of two different processes: model validation and sensitivity analysis of the modeling strategy.

\subsubsection{Model Validation}

Restricted by the absence of in-house experimental data for RDC, the validation of the selected numerical strategy was based on theoretical and experimental data previously reported in the literature. Therefore, the numerical predictions obtained in the present study were compared with analytical solutions or with experimental data, based on their availability. Using the results from the model validation, different modeling parameters were selected. The selection of mesh size, time step and discretization schemes was based on the criteria to obtain accurate predictions while maintaining acceptably low computational cost.

\subsubsection{Sensitivity Analysis of the Modeling Strategy}

As part of the present study, the response of the validated modeling strategy to geometric changes and operating conditions was investigated. This was achieved by performing numerical simulations under conditions different than those for the bench mark cases. It was ensured that the simulations maintained generality and predicted the expected trends. Additionally, the stability of the model under increased outlet pressure conditions was maintained .

An iterative procedure between model validation and sensitivity analysis of the model was performed. Feedback from the sensitivity analysis into the model validation permitted appropriate generality of the proposed modeling strategy. 


\subsection{Objectives}

In the present work, a numerical study of rotating detonation combustion has been performed under operational conditions relevant to stationary power generation, with the following main objectives:

1. To develop a strong, robust and accurate modeling strategy for the simulation of RDC with the capability of accurately predicting the main performance characteristics relevant to gas turbine applications.

2. To determine appropriate geometrical configurations under which continuous detonation combustion could be achieved for operating conditions relevant to gas turbine applications.

3. To predict limiting conditions for continuous detonation and maximum pressure gain. 


\section{Chapter 2}

\section{Literature Review}

Rotating detonation combustion presents a complex interaction of fluid flow and chemical phenomena. Most of these interactions are governed by the presence of one or multiple detonation waves inside a semi-enclosed combustion chamber. The understanding of the basic physical processes regarding detonation waves is necessary to have an adequate appreciation of the processes taking place in RDC. Section 2.1 presents a short description of the detonation phenomena, and a comparison between deflagration and detonation combustion. Additionally, a brief review of the analytical models used to predict detonation wave parameters is included. Additionally, an introduction to the Chapman-Jouguet (CJ) model and the Zel'dovich-Neumann-Döring (ZND) is given.

In Section 2.3 a review of some relevant experimental studies for RDC is presented. Based on a chronological approach, a description on the main advances in RDC experimental techniques and set ups is provided, along with a summary of the geometrical and operating parameters for the selected studies. Finally, Section 2.4 provides a summary of theoretical and numerical studies on RDC. Special attention is given to the model assumptions and modeling strategies taken from the different numerical studies in the literature.

\subsection{Detonation Phenomena}

During the combustion process, the chemical potential energy stored in the fuel and oxidizer molecules is transformed into heat through a sequence of exothermic chemical reactions. For premixed combustion, this conversion occurs in a relatively thin reaction front. This front propagates in a gaseous mixture as a combustion wave. In a premixed reactive medium this propagation can can occur as two distinct combustion regimes: deflagration or detonation combustion, which are classified depending on the propagation velocity of the reaction front. Deflagration 
waves (also referred to as flames) propagate at low subsonic velocities $\left(10^{-2}-10^{1 / m} / s\right)$, where diffusion of mass and heat from the reaction front into the un-reacted mixture permits selfsustained combustion. In other words, the deflagration waves propagate due to the upstream influence of the reaction front on the premixed reactants. The velocity at which a planar laminar deflagration front propagates is defined as the Laminar Flame Speed $\left(S_{L}\right)$. The laminar flame speed can be determined through experimental studies, theoretical simplified formulations and detailed calculations. The calculation of $S_{L}$ and the flame structure requires detailed knowledge of the chemical kinetics and transport properties of the reactive mixture and the combustion products. The second regime of combustion is called detonation combustion. In this regime the reaction front propagates at supersonic velocities in the order of $10^{3 \mathrm{~m}} / \mathrm{s}$. In detonation combustion, a shock wave travels in front of a thin reaction region. The leading shock wave increases the temperature and pressure of the unreacted mixture, inducing the formation of radical species and promoting the formation of combustion products. Following the induction region, a heat release zone develops, where the un-reacted fuel and air react to form detonation products. Figure 2.1 presents a comparison between the front structure for planar deflagration and planar detonation wave for combustion of stoichiometric $\mathrm{H}_{2}$-Air. The temperature and pressure are scaled by arbitrary factors in order to favor appropriate visualization.

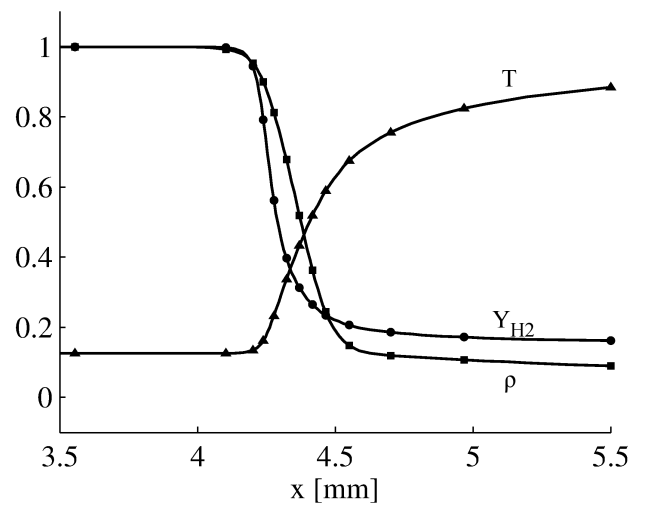

(a)

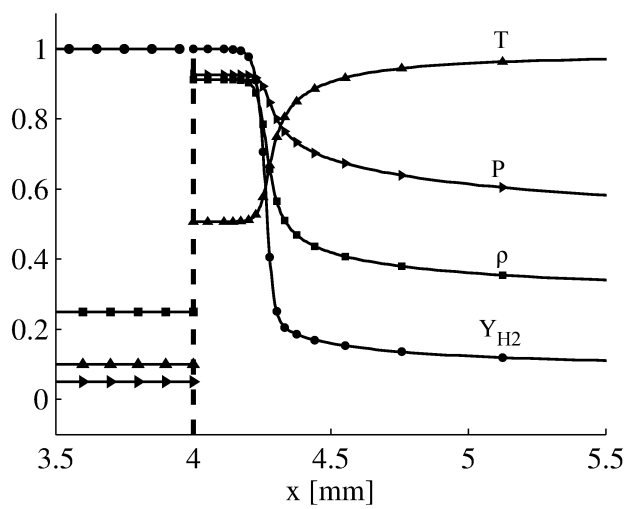

(b)

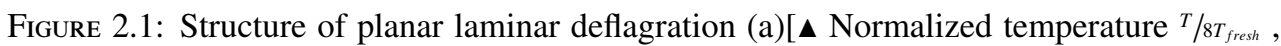
normalized density ${ }^{\rho} / \rho_{\text {fresh }}$, normalized $H_{2}$ mass fraction $\left.{ }^{Y_{H_{2}}} / Y_{H_{2}, \text { fresh }}\right]$ and planar detonation (b) [^ Normalized temperature ${ }^{T} / 8 T_{\text {fresh }}$, normalized density ${ }^{\rho} / \rho_{\text {fresh }}$, normalized $\mathrm{H}_{2}$ mass fraction $Y_{H_{2}} / Y_{H_{2}, \text { fresh }}$, and $\triangleright$ normalized pressure $\left.{ }^{P} / 20 P_{\text {fresh }}\right]$

In addition to the difference in the propagation velocity of the reaction front, deflagration and detonation present fundamental differences in their planar structure. The upstream influence of the reaction front in deflagration combustion leads to a relatively smooth change in the state of the reactive mixture. On the other hand, detonation combustion presents a nearly discontinuous change of the thermodynamic state of the mixture due to the presence of the preceding shock wave. In deflagration combustion, the temperature increases gradually as fuel is consumed and heat is added to the mixture. Under adiabatic conditions, the temperature reaches its maximum 
value once the fuel is totally consumed; this temperature is referred as the adiabatic flame temperature $\left(T_{f, a d}\right)$. In contrast, for detonation combustion the temperature increases in two stages: First, the temperature increases due to the shockwave compression effect. After this compression a near isothermal region developes. This zone is called the induction region, and it is where radical species are formed and chemical reactions are induced. Second, the heat release occurs after the induction region, leading to further temperature increase. The plateau behavior in the detonation wave structure can also be observed on the mixture temperature, density, pressure, and major species distribution (see Figure 2.1(b)). Moreover, it is important to highlight that the most relevant difference is with regards to the pressure. During deflagration combustion, pressure does not change significantly. The process is considered isobaric and the model used to calculate the planar deflagration structure in Figure 2.1 considers the pressure to remain at its initial value. On the other hand, the existence and maintenance of the detonation wave relies on the presence of a strong shock wave. As seen in Figure 2.1, the pressure increases behind the strong shock wave, then an isobaric zone corresponding to the induction region is formed, and the detonation products expand away from the reaction front. It is observed that in detonation combustion, the density follows the same qualitative behavior as the pressure. More details on the difference between deflagration and detonation combustion can be found in [Kuo, 1986]; a summary of these differences is presented in Table 2.1, which was modified from [Kuo, 1986].

TABLE 2.1: Deflagration and Detonation combustion comparison [Kuo, 1986]

\begin{tabular}{ccc}
\hline \hline & Deflagration & Detonation \\
\hline$P_{o} / P$ & 0.98 & $13-55$ \\
$T_{o} / T$ & $4-16$ & $8-21$ \\
$\rho_{o} / \rho$ & $0.06-0.25$ & $1.7-2.6$ \\
\hline
\end{tabular}

Great advancements on the understanding of detonation fundamentals have been achieved since the late $19^{\text {th }}$ century. The development of the analytical model to calculate the thermodynamic state and composition for the detonation products is attributed to Donald Leonard Chapman and Ehrile Jouguet [Lee, 2008]. The Chapman-Jouguet (CJ) Model neglects the structure of the detonation wave, and calculates the detonation state based on the conservation equations ahead and behind the detonation wave. Details on the derivation of the $\mathrm{CJ}$ model are presented in [Hirschfelder et al., 1954, Law, 2006, Lee, 2008], while the methodology for the calculation of CJ detonation is presented in [Kuo, 1986]. Computer programs such as [Gordon and McBride, 1972] and [Goodwin, 2005] provide an efficient and easy to use tool to calculate the CJ state of multiple reactive mixtures. Figure 2.2 shows the calculation of the $\mathrm{CJ}$ state for Hydrogen-Air at different equivalence ratios $(\Phi)$. The calculations were performed using [Goodwin, 2005]. It is observed how the CJ detonation wave velocity $U_{C J}$ increases with $\Phi$ and with the pressure ahead of the reaction front $\left(P_{\text {fresh }}\right)$ pressure of the mixture. A similar behavior is observed for the estimated CJ temperature $\left(T_{C J}\right)$, but a slight decrease occurs after stoichiometric condition $\Phi=1.0$. The $\mathrm{CJ}$ pressure $\left(P_{C J}\right)$ on the other hand, is nearly independent of $\Phi$, but instead is a 
strong function of the pressure ahead of the detonation wave. Despite of the simplicity of the CJ model, this calculations are of great relevance to the present study, and provide valuable initial insight to the behavior of detonation combustion at elevated pressures. These results will be revisited in the following sections.

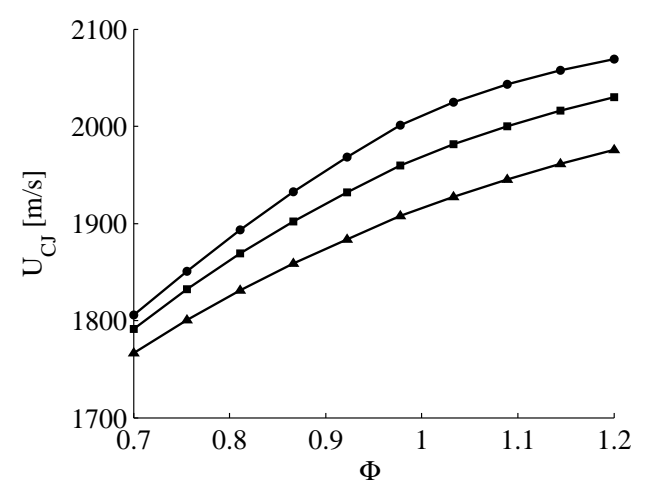

(a)

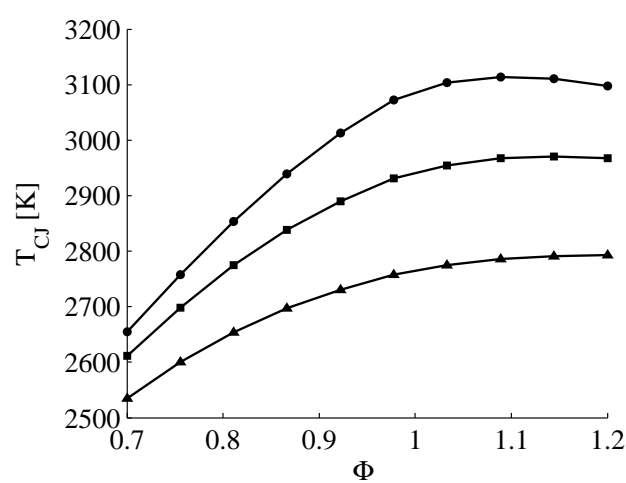

(b)

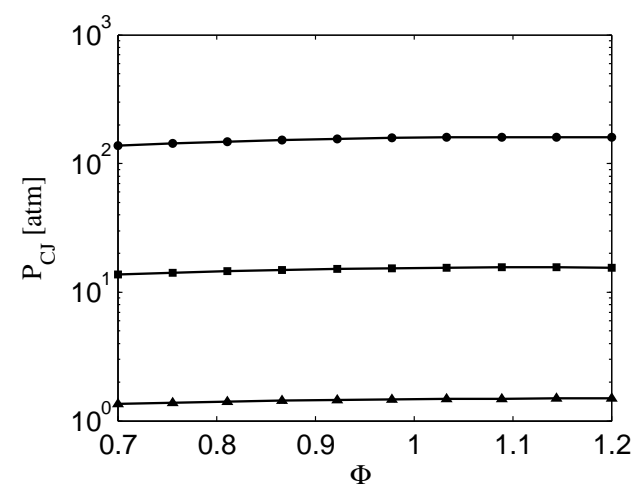

(c)

FIGURE 2.2: Chapman-Jouguet conditions for $\mathrm{H}_{2}$-air mixture: Velocity (a), Temperature (b), and Temperature (c) for $\mathrm{H}_{2}+$ Air at initial pressures of: $\left[\boldsymbol{\Lambda} P_{\text {fresh }}=0.1 \mathrm{~atm}, \boldsymbol{\square} P_{\text {fresh }}=1.0 \mathrm{~atm}\right.$,

$$
\left.P_{\text {fresh }}=10.0 \mathrm{~atm}\right]
$$

Chapman-Jouguet calculations provide important information on the thermodynamic state of detonation products; however, they fail to consider the structure of the detonation wave. The widely adopted theoretical model to calculate the detonation wave structure for planar steady detonation is accredited to Yakovich B. Zeldovich, John von Neumann and Werner Döring[Lee, 2008]. The Zeldovich-Neumann-Döring (ZND) model assumes that the detonation wave has a planar and steady structure as presented in Figure 2.1(b). In order to calculate the ZND structure of detoantion waves it is required to specify the chemical kinetic mechanism for the mixture. The ZND model equations can be solved through a numerical strategy found in [Lee, 2008]. Several computational tools have been developed to calculate the ZND structure in gaseous mixtures, such as [Design, 2013] and [Goodwin, 2005].

The ZND model provides enough information to calculate some of the governing length and temporal scales of the detonation wave. For example, the characteristic induction length 
$\Delta_{I}$, reaction length $\Delta_{R}$, the distance to the CJ plane $\Delta_{C J}$ and their correspondent time scales $\tau_{R}, \tau_{I}$ and $\tau_{C J}$ can be calculated using the information from the ZND structure. As shown in Figure 2.3, $\Delta_{I}$ is defined as the distance from the leading shock front to the maximum value of thermicity. The thermicity $(\dot{\sigma})$, as defined by [Ng et al., 2005] and given in Equation 2.1, denotes the normalized chemical heat release rate. It is then possible to define the characteristic time scale for heat release $\left(\tau_{R}\right)$ as the inverse of $\dot{\sigma}_{\max }$ [Ng et al., 2005], therefore $\Delta_{R}=U_{c J} / \dot{\sigma}_{\max }$.

$$
\dot{\sigma}=\sum_{k=1}^{N_{k}}\left(\frac{M w_{m i x}}{M w_{k}}-\frac{h_{k}}{C p_{m i x} T}\right) \frac{\partial Y_{k}}{\partial t}
$$

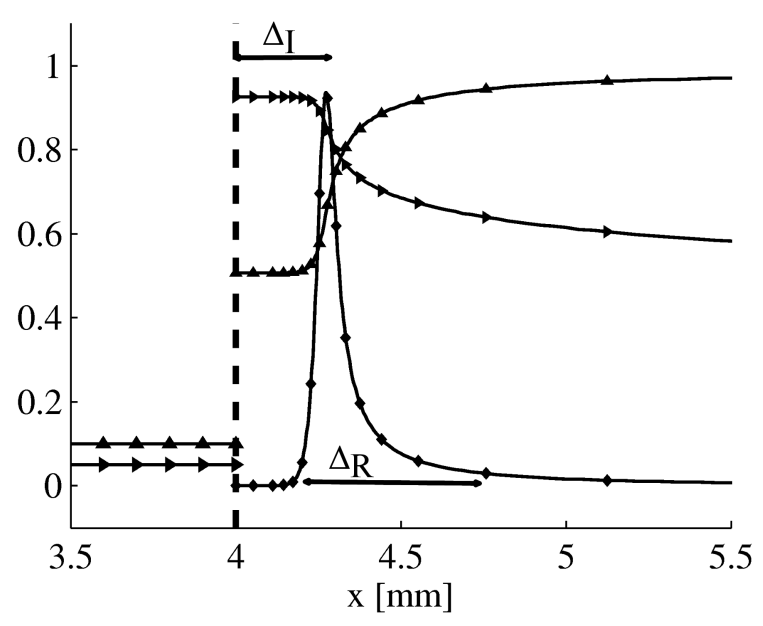

FIGURE 2.3: Length scales for ZND detonation wave [ $\boldsymbol{\Lambda}$ Normalized temperature ${ }^{T} / 8 T_{\text {fresh }}$, normalized pressure ${ }^{P} / 20 P_{\text {fresh }}, \diamond$ normalized thermicity $\left.\dot{\sigma} / 3 \times 10^{6}\right]$

Although the ZND model assumes that detonation wave structures are planar and stable, detonation waves are a multidimensional and unstable phenomena. One dimensional instability of detonation waves can be studied using modified ZND models [Ng et al., 2005], but in order to capture the multidimensional nature of the flow, experimental and numerical studies are required. Experiments by [Radulescu et al., 2007] show Schlieren photography, where evidence of the cellular instabilities in the detonation wave are observed. The intricate interaction among the leading shock wave, the transverse waves and reaction fronts lead to the characteristic cellular structure of detonation wave propagation. An additional characteristic length scale is determined from the cellular structure of detonation wave. This length scale is often referred to as the detonation cell size $\left(\lambda_{D}\right)$. It is defined as the distance between two adjacent triple points in the detonation wave structure. $\lambda_{D}$ is measured experimentally using soot record on the walls of a detonation chamber [Lee, 2008] or using high resolution schilieren pictures [Shepherd, 2009]. $\lambda_{D}$ is a function of the reactive mixture, its state ahead of the detonation wave, and the stability of the detonation front.

Several numerical studies have been performed in order to predict the details of detonation wave instability and the multidimensionality of its front. For example, a study on the effect 
of mesh resolution on the prediction of the detonation wave structure performed by [Sharpe, 2001], showed that in order to capture the details of detonation wave structure at least 50 computational cells are required in the length of the heat release zone or reaction length $\left(\Delta_{R}\right)$. This means that in the case of stoichiometric $\mathrm{H}_{2}$-Air detonations, a mesh resolution in the order of $1 \mu \mathrm{m}$ is required. Numerical simulations of the $3 \mathrm{D}$ detailed structure of detonation waves have also been performed by [Dou et al., 2008, Tsuboi et al., 2007]. These studies are carried in relatively small computational domains, with a maximum length of 20-30 $\Delta_{R}$. Mesh resolution for 3D simulation of detailed detonation front is of around 20 elements in $\Delta_{R} / 2$. The numerical resolution required to capture the intricate interaction of the detailed detonation front is very high, and such levels of resolution are not practical for the numerical simulation of RDC with the computational resources available for the present study.

\subsection{Thermodynamic Efficiency of Detonation Combustion in Power Cycles}

Recent research efforts to increase gas turbine thermodynamic efficiency have focused mostly on increasing the capability of gas turbine hardware to withstand higher turbine inlet temperatures. Advancements in cooling strategies, manufacturing processes, and thermal barrier coatings have allowed the thermodynamic efficiency to be increased up to $40 \%$ [Saravanamuttoo et al., 2001]. However, great difficulty is observed in achieving further improvements without incurring high cost or complexity. An alternative solution is the implementation of a different thermodynamic cycle leading to higher efficiency.

Heat addition for conventional simple cycle gas turbine occurs through the turbulent combustion of a reactive mixture. As covered in Section 2.1, the process of deflagration is characterized as a relatively constant pressure process. Therefore, the ideal thermodynamic cycle to represent conventional gas turbine operation is the Brayton cycle [Saravanamuttoo et al., 2001]. As depicted in Figure 2.4 [Russo et al., 2011], the Brayton cycle consists of isentropic compression from the initial state 0 to state 1 , then constant pressure heat addition occurs from state 1 to state 4 followed by isentropic expansion from states 4 to 5 , and the cycle is closed by a fictitious constant pressure heat discharge back to the initial state 0 . A different ideal cycle is used to consider constant-volume-combustion operation, the Humphrey cycle. In comparison with the Brayton cycle, the Humphrey cycle consists of isentropic compression from initial state 0 to state 1 followed by a constant volume heat addition that leads to further increase pressure to state 2 , then isentropic expansion occurs from state 2 to state 3 followed by a constant pressure heat discharge back to the initial state 0 . 

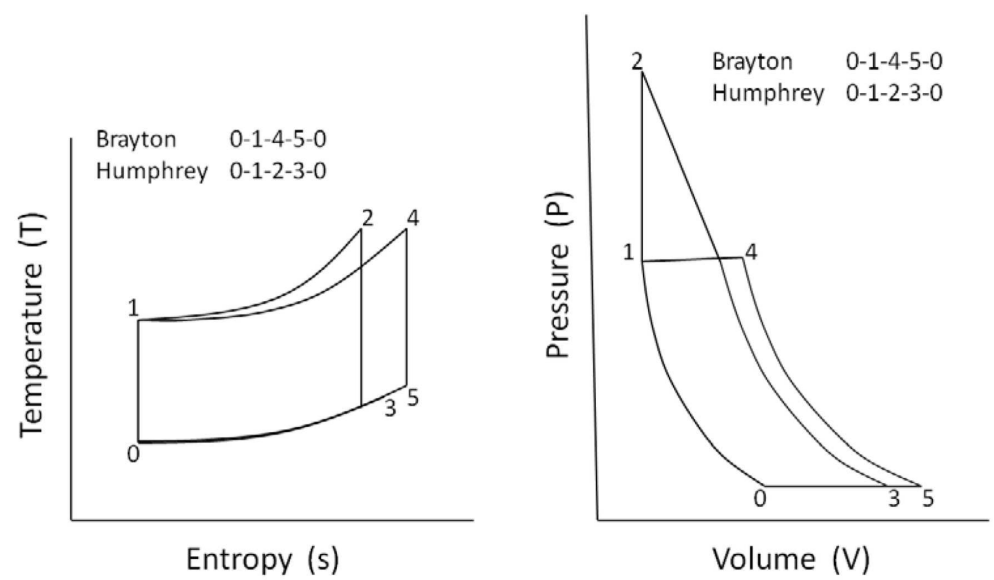

FiguRE 2.4: Temperature-Entropy (left) and Pressure-Volume (right) for Brayton and Humphrey cycles taken [Russo et al., 2011]

The thermodynamic efficiency for the Brayton cycle $\eta_{B}$ is determined by the temperature ratio of state $1 T_{1}$ over initial temperature $T_{0}$. The efficiency for Humphrey cycles $\eta_{H}$ takes into account the temperature ratio of states after $T_{2}$ and before the constant volume heat addition $T_{1}$ and generally leads to higher thermodynamic efficiency. $\eta_{B}$ and $\eta_{H}$ are calculated using Equation 2.2 and Equation 2.3, respectively.

$$
\begin{gathered}
\eta_{B}=1-\frac{T_{0}}{T_{1}} \\
\eta_{H}=1-\frac{T_{0}}{T_{1}} \gamma\left[\frac{\left(\frac{T_{2}}{T_{1}}\right)^{1 / \gamma-1}}{\frac{T_{2}}{T_{1}}-1}\right]
\end{gathered}
$$

Although the Humphrey cycle has been widely adopted as a surrogate cycle for the representation of pulse detonation engine (PDE), recent analysis by [Vutthivithayarak et al., 2012] shows that under prediction of the thermodynamic efficiency for constant volume combustion cycles can be occur through the Humphrey cycle. Within the work by [Vutthivithayarak et al., 2012] the description of Ficket-Jacobs $(F J)$ and ZND is provided and its performance compared with that for the Humphrey cycle. Figure 2.5 shows the pressure-volume and temperature entropy diagrams for Humphrey, FJ and ZND cycles for stoichiometric H2-Air.

The main difference between these the Humphrey, FJ and ZND cycles is encountered in the processes of constant volume compression and heat addition. The Humphrey cycle assumes isochoric compression from the initial state 1 to the reactive Hugoniot line. In comparison, the FJ cycle considers that the change from the initial state 1 to the Chapman-Jouguet (CJ) state $2 \mathrm{CJ}$ occurs through a Rayleigh heating process. On the other hand the ZND cycle considers the structure of the detonation wave, where shock compression occurs through the non-reactive 


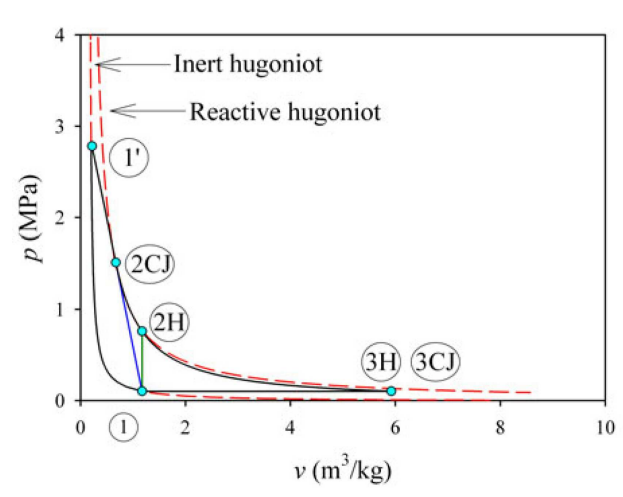

(a)

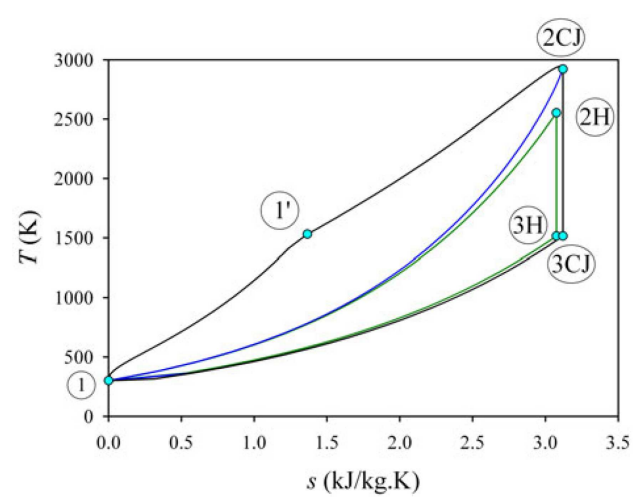

(b)

FIGURE 2.5: Humphrey, FJ and ZND cycles for stoichiometric $H_{2}$-Air. Pressure-specific volume diagram (a) and temperature-entropy diagram (b) for Humphrey $(1 \rightarrow 2 \mathrm{H} \rightarrow 3 \mathrm{H} \rightarrow 1)$, FJ $(1 \rightarrow 2 \mathrm{CJ} \rightarrow 3 \mathrm{CJ} \rightarrow 1)$ and $\mathrm{ZND}\left(1 \rightarrow 1{ }^{\prime} \rightarrow 2 \mathrm{CJ} \rightarrow 3 \mathrm{CJ} \rightarrow 1\right)$

Hugoniot line taking the gas to the von-Neumann state (1'). At this state exothermic reaction of the reactive mixtures occur and heat is added through a Rayleigh process taking the gas to its CJ state (2CJ). For all the Humphrey, FJ and ZND cycles, an isentropic expansion process is considered to take gas back to the pressure value of state 1 and then a fictitious heat discharge process at constant pressure brings the gas to its initial state 1 . Thermal efficiencies for the cycles depicted in Figure 2.5 are of 66.5\%, 64.3\% and 70\% for Humphrey, FJ and ZND cycles respectively. As stated by [Vutthivithayarak et al., 2012], the Humphrey cycle fails to capture the physics of detonation processes and underestimates the work performed by the constant volume cycle. This leads to lower predictions of thermodynamic efficiency.

Thermodynamic processes occurring during RDC have proved to be of great interest to the scientific community. The presence of flow structures different than detonation waves raises the concern on which ideal thermodynamic cycle best describes RDC. As shown in the work performed by [Nordeen et al., 2011]. different sections of the flow incur different thermodynamic cycles. According to their research. the flow can be classified in three regions depending on the characteristic thermodynamic processes they experience. The first region or main flow consists of streamlines that pass through the detonation wave and then experience isentropic expansion. This region comprises approximately $68 \%$ (by mass) of the flow. The second region is formed by streamlines that after passing through the detonation wave encounter the shock wave formed by the interaction of the detonation wave and the combustion products (see oblique shock wave shown in Figure 1.3). Passing through this shock wave leads to increase in entropy and losses in total pressure. The second flow region is of -approximately- $18 \%$ of the flow. The third group includes streamlines that go through a constant pressure heat addition processes on the combustion front formed by the interaction of injected mixture and detonation products. Approximately $4 \%$ of the flow is consumed through a deflagration process leading to performance losses. As stated in [Nordeen et al., 2011] the percentages are representative of the RDC regime, but are 
dependent on the combustion chamber geometry and injection parameters. Quantitative disagreements between the modified ZND cycle proposed by [Nordeen et al., 2011] and numerical predictions were seen. These were attributed to deficiencies of the chemical modeling strategy adopted in the numerical model. It was then concluded that a modified ZND cycle, taking into account the incoming velocity of injected reactant, appropriately predicted the core of RDC flow.

The works cited in Section 2.2 support an expected increase in thermodynamic efficiency when operating under a ZND cycle instead of a Brayton cycle. One aim of the current study is to reveal if it is possible and under which conditions these improvements can be achieved.

\subsection{Experimental Studies of Rotating Detonation Combustion}

Origins of RDC can be traced back to the experimental work performed in the Hydrodynamic Institute, Siberian Branch of the Russian (USSR) Academy of Science by Voitsekhovsky in the late 1950's [Voitsekhovsky, 1960]. The experimental set up leading to RDC was designed in order to study the characteristics of standing detonation waves. The combustion chamber consisted of a disk shaped chamber. In this chamber the reactants would enter in the radial direction and encounter a detonation wave traveling in the azimuthal direction. The detonation wave front traveled parallel to the axis of the chamber. Combustion products expanded outward in the radial direction to be dumped to an exhaust tank at an average pressure of $0.75 \mathrm{~atm}$. Premixed injection of acetylene and oxygen was used in this study and ignition of the system was performed through high energy discharge. Although it is not specified in the study, it is most likely that this high energy discharge was provided through a detonation cap or a similar small explosive device. Unidirectionality of the detonation wave was achieved through the temporal blockage of the chamber during the first cycle of the detonation wave. High complexity in the initiation procedure and the need of accurately timing to guarantee continues detonation is evident from the early stages of RDC, and continues to be a very important aspect of experimental studies.

Along with proving the concept of continuous detonation or RDC, experimental strategies for image recording of the process were established by Voitsekhovsky. Analysis of the images taken by [Voitsekhovsky, 1960] permitted the description of the main flow structures encountered in RDC.

Problems with flame flash back were encountered by Voitsekhovsky due to the use of premixed fuel oxidizer injection to the combustion chamber. This set the path for changes in RDC configurations. Consequently, as presented in [Bykovskii and Mitrofanov, 1980], Bykosvkii 
used an experimental set up where the fuel $\left(\mathrm{C}_{2} \mathrm{H}_{4}\right)$ and oxidizer $\left(\mathrm{O}_{2}\right)$ were injected from separate streams. The applied injection stagnation pressure ranged from $5-8 \mathrm{~atm}$. The reactants were injected at the inlet end of an annular chamber with an inner diameter $\left(D_{i n}\right)$ of $40 \mathrm{~mm}$, outer diameter $\left(D_{\text {out }}\right)$ of $50 \mathrm{~mm}$ and $100 \mathrm{~mm}$ in length $\left(L_{c}\right)$. The detonation products expanded in the azimuthal and axial directions, leaving the combustion chamber with a predominant axial component to an exhaust tank at an initial pressure of $0.03 \mathrm{~atm}$. For certain experimental runs, an annular ring was placed at the outlet of the combustion chamber to study the influence of partial blockage of the outlet on RDC performance.

In [Bykovskii and Mitrofanov, 1980], the initiation of unidirectional detonation wave was performed through the usage of an initiation tube placed near the injection plenum and tangential to the combustion chamber. A highly reactive mixture was placed in the initiation tube, and after it was ignited, the reaction front would transition from deflagration to detonation. The resultant detonation wave would enter the chamber with a preferable direction.

It is reported in [Bykovskii and Mitrofanov, 1980] that the presence of the blockage and the influence of the reflected shock wave decreases the detonation wave velocity. These observations are of great relevance to the work considered in this document since they prove that an inappropriate treatment of the outlet condition leading to reflected pressure waves can significantly harm numerical predictions.

A study on the fuel injection location and the influence of conical expansion of the inner wall for RDC cylindrical chambers was also performed by [Bykovskii and Vedernikov, 2003]. An experimental set up with various combustion chamber geometries was studied. The $D_{\text {in }}$ was varied from $30-90 \mathrm{~mm}, D_{\text {out }}$ from $40-100 \mathrm{~mm}$ and $L_{c}$ from $100-200 \mathrm{~mm}$. Cylindrical chamber with constant cross sectional area and a chamber with $8.5^{\circ}$ of axial expansion for the inner diameter were studied. The photo recording of the detonation wave and accompanying structures in [Bykovskii and Vedernikov, 2003] was taken through a narrow slot on the outer cylinder of the chamber. They established different operational regimes for RDC based on the combustion chamber geometry. It was observed that longitudinal oscillations because of the periodic displacement of the combustion front can occur due to the expansion of the combustion chamber. In this regime, the combustion products move upstream behind the detonation wave with a subsequent entrainment downstream. Therefore, the detonation regime acquired a pulse detonation behavior in the axial direction.

In the work presented by [Bykovskii and Vedernikov, 2008], an extensive and detailed summary of experimental techniques employed for RDC characterization is given. Bykovskii provides the calculation methods for mass flow rates into the combustion chamber based on pressure measurements of reactants reservoirs. Additionally, suggestions on pressure transducers to be used and techniques to minimize noise on pressure readings are explained in detail. One of the most relevant aspect in their study is the explanation of the photo recording technique 
and the methodology used to analyze the image data in order to obtain the velocity distribution inside the combustion chamber.

Information on the temperature distribution and heat flux experienced in a cylindrical combustion chamber under RDC regime is reported by [Bykovskii and Vedernikov, 2009]. In their study, multiple combustion chamber configurations were tested. A constant cross sectional area combustion chamber with length of $665 \mathrm{~mm}$ and a conical combustion chamber with $8.5 \mathrm{deg}$ expansions were tested. Acetylene air and hydrogen air were injected into the chamber with a stagnation injection pressure of $15 \mathrm{~atm}$, and the combustion products were exhausted to the atmosphere. Additional to RDC heat flux measurements, data was taken for turbulent combustion in the same combustion chambers. The measured RDC heat fluxes varied depending on the axial location of the combustion chamber, and the time of operation. It was observed that the maximum heat flux region correlated with the region of heat release, determined by the height of the detonation wave. The periodic cooling effect of the injected reactants was observed. Average heat fluxes ranged between $1.2-1.5^{\mathrm{MW}} / \mathrm{m}^{2}$, representing close to $0.5 \%$ of the total enthalpy introduced by the reactants. Measurements for RDC and turbulent combustion were in the same scale range.

Recent experimental and numerical research on RDC for gas turbine applications has been performed by researchers at (Warsaw University of Technology) in collaboration with the Warsaw Institute of Aviation. For instance the study presented in [Kindracki et al., 2011a], shows experimental data for RDC on different combustion chamber configurations. In their study, continuous detonation was obtained for annular chambers with $D_{\text {out }}=95 \mathrm{~mm}, 150 \mathrm{~mm}$ and $134 \mathrm{~mm}$, for which pressure and temperature measurements were reported. Additionally, the process of self stabilization for continuous detonation was described. Although the author mention that the experimental set up used had the capability of operating at exhaust pressures from 0.05 bar to $2.5 \mathrm{bar}$, the experimental data for outlet pressures greater than 1 bar was not presented. The experimental work was accompanied by 2D and 3D simulations of rotating detonation combustion, with appropriate prediction of the detonation wave velocity and pressure history of the recorded detonation wave.

The research project under which the present $\mathrm{PhD}$ thesis was developed is titled Simulation and Model Validation of Energy Technologies. This project was performed in collaboration with the National Energy And Technology Laboratory (NETL), in Morgantown, WV. As part of this project, The Thermal Science Division at NETL constructed an experimental set up to study the behavior of RDC at outlet pressures relevant for gas turbine applications. This facility will have the capability of reaching exhaust pressures as high as 8atm at the combustor outlet. The combustion chamber consists of an annular section that is enclosed between two concentric cylinders: the Outer Cylinder and the Center Body insert, with $D_{\text {out }}=150 \mathrm{~mm}$ and $D_{i n}=140 \mathrm{~mm}$, respectively. The combustion chamber has a length $\left(L_{c}\right)$ of $100 \mathrm{~mm}$. An external 
initiation tube is used to introduce a detonation wave in the annular chamber with a preferable propagation direction. An isometric view of the combustion chamber and attached subsystems is provided in Figure 2.6(a), while the details of the internal geometry and fuel and oxidizer flow paths are shown in the sectioned view Figure 2.6(b). The light blue arrow depicts the flow path of the oxidizer, the yellow arrow shows the path of the fuel, and the red arrow shows the general path that the detonation products follow as the expand towards the outlet of the combustion chamber. The four lateral perforations on the outer cylinder are access ports for high sampling rate pressure transducers. The pressure transducers will be placed in these ports in order to determine the occurrence of detonation and to measure its propagation velocity. They are distributed in two axial arrays, separated by $180 \mathrm{deg}$ in the combustion chamber.

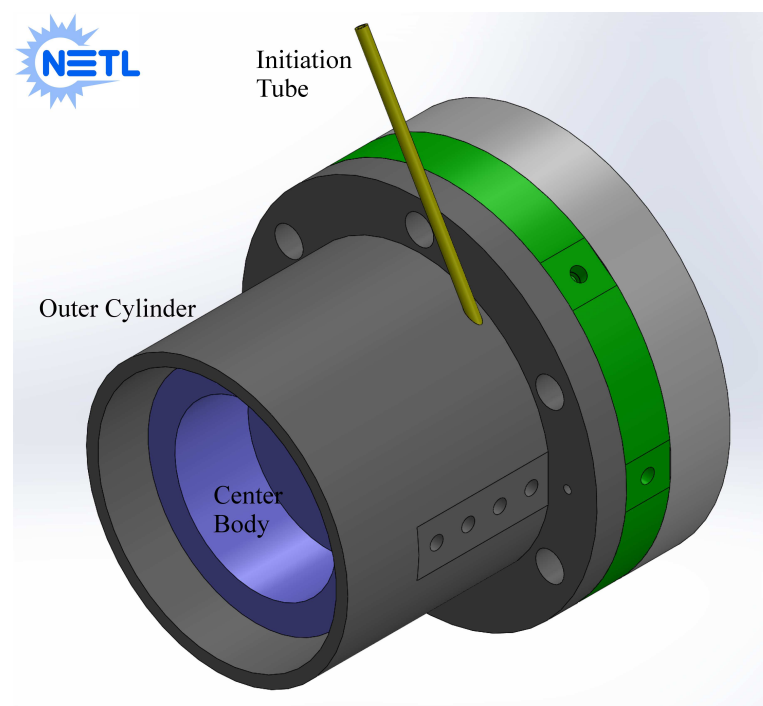

(a)

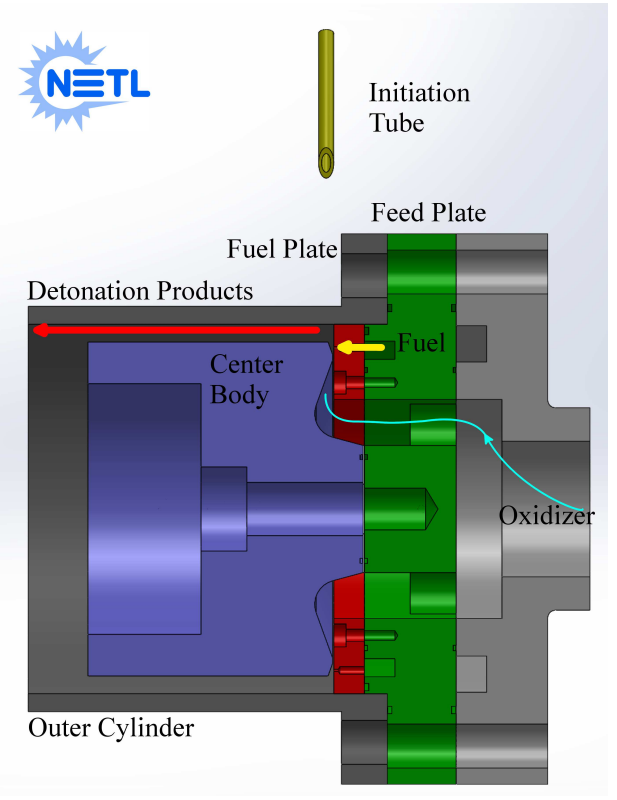

(b)

FIGURE 2.6: NETL RDC experimental set up. Isometric view (a), sectioned view (b)

Based on [Ferguson, 2014], the experimental procedure is started by flowing an inert gas $\left(N_{2}\right)$ into the system until the desired operating pressure is reached. The outflow of the system and back pressure are controlled by a valve at the outlet of the experimental set up. Once the required pressure is achieved, the fuel and oxidizer are injected into the system. Initial experiments will be performed for $\mathrm{H}_{2}$-Air mixtures [Ferguson, 2014]. Additionally, $\mathrm{H}_{2}+\mathrm{Air}$ is injected into the initiation tube. The mixture in this external device is then ignited using a spark plug located in its front end. It is expected that the initiated deflagration transitions into detonation before reaching the annular chamber. In order to guarantee initiation, the spark plug is activated four times in the lapse of 1 second, and after this first second the flow of $H_{2}+$ Air is stopped. If the initiation process is successful, the presence of a detonation will be verified using the data from the pressure transducers and $\mathrm{OH}$ sensors. Additionally, a mirrors arrangement with high speed camera will provide visual access to the outlet of the combustion chamber. 
Experimental measurements for RDC are highly complex and expensive. The high pressures, and temperatures characteristic of detonation combustion, along with the short characteristic time scales, provide stringent requirements on measurement techniques and equipment. Therefore, analytical and computational techniques have been use to study RDC since the early 1960's. The following section (Section 2.4) provides the reader with a short review of the past analytical and numerical work performed on RDC.

\subsection{Numerical and Analytical Studies of Rotating Detonation Com- bustion}

Development of theoretical models to describe RDC have been closely related to the experimental advancements presented in the previous subsection (Section 2.3). As a first available approximation, Voitsekovskii in [Voitsekhovsky, 1960] developed a one dimensional model that enables the calculation of velocity, pressure and density of the flow at various regions of RDC. However, the predictions from this model rely on the experimental data obtained from the photo recording of the detonation and shock wave patterns.

Parallel to the work performed by Voitsekhovsky for the Hydrodynamic Institute in Siberia, similar research was carried out in the US by Nichols and his research team from the Aircraft Propulsion Laboratory at the University of Michigan. They performed a feasibility study of RDC for propulsive applications during the early sixties [Nicholls and Cullen, 1964]. Under a contract with the Air Force Flight Test Center, experimental and analytical studies were carried out. The experimental efforts did not lead to a configuration capable of maintaining continuous detonations. However, analytical models for gaseous and gaseous-liquid RDC were developed. The analysis resulted in 1D models capable of predicting the local state of the products and reactants in the azimuthal direction of the RDC annular chamber.

The model developed by [Nicholls and Cullen, 1964] is used in the present work for two purposes: First, it is used as a preliminary predicting tool to determine the conditions under which sustained continuous detonation is expected; and second, it is used to calculate an initial condition suitable for the performance of transient numerical simulations. A short description of this model is provided next.

In the work by [Nicholls and Cullen, 1964] three types of analytical models for rotating detonation combustion were developed: The first model assumes complete and immediate mixing of the detonation products and the incoming reactant mixture. The second model is based on the assumption that there is no mixing between the detonation products and the reactants, and that they are separated by a discrete interface. The third model also considers non-mixing, but it incorporates the presence of liquid droplets of cryogenic fuels. The experimental and numerical 
results obtained during the last two decades determine the existence of a discrete combustion front between the incoming reactants and the products of the detonation wave. Therefore, it is expected that the model developed under the non mixing approach provides a better prediction of the RDC operation. In the present study, the second model was modified and implemented. This model is based on the assumption that the variation of the flow quantities along the axial direction of the combustor can be neglected. It is also assumed that the interface between fresh reactants and detonation products is a streamline, where there is no heat, mass or momentum transport across it. This streamlines divides the combustion chamber into two distinct control volumes, where one belongs to combustion products and the other to fresh reactants. A schematic representation of the control volumes is presented in Figure 2.7. For the development of this model, the combustion chamber is simplified onto a planar domain were two distinct control volumes, $\mathrm{R}$ and $\mathrm{P}$, are defined. The control volume $\mathrm{R}$ corresponds to the non reacted mixture of fuel and oxidizer, while $\mathrm{P}$ represents the detonation products. The location $\theta=0$ corresponds to the point immediately behind the detonation wave. The horizontal arrows in Figure 2.7 that point from right to left represent the velocity of the gases in the wave fixed frame of reference. In this model it is assumed that all the reactants mass injected at the inlet are transformed into products by the detonation wave. The detonation products expand behind the detonation wave and exit the combustion chamber through the outlet.

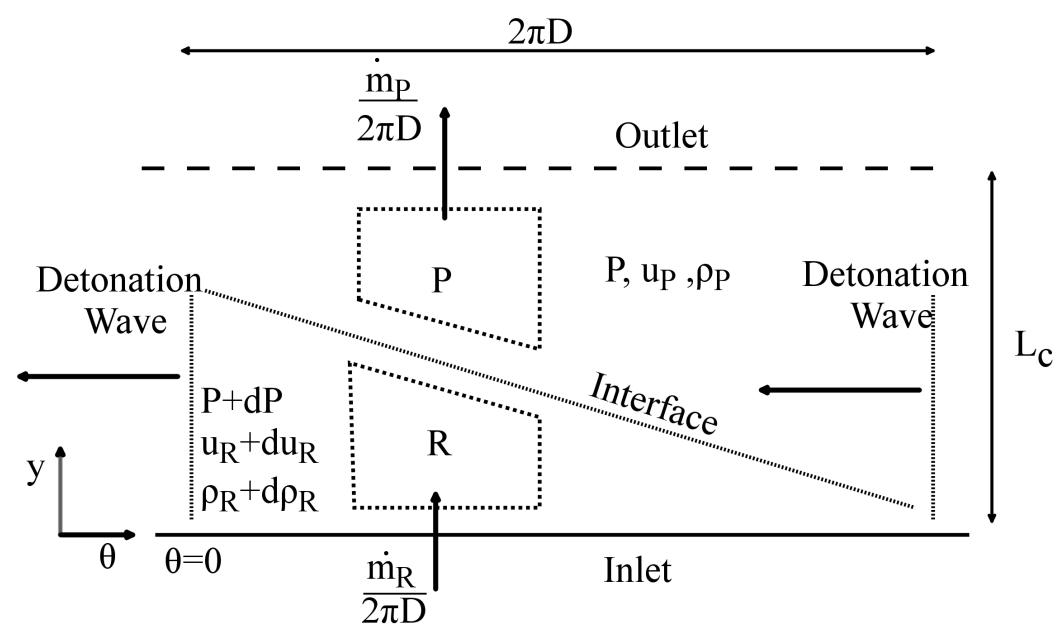

FIGURE 2.7: Control volume schematic for analytical 1D RDC model, after [Nicholls and Cullen, 1964]

Based on the control volume formulation shown in Figure 2.7, the equations of continuity, energy and momentum are integrated for the reactants $(R)$ and the products $(P)$. Details of the integration can be found in [Nicholls and Cullen, 1964]. The resulting equations are then normalized with respect to the state of the products at $\theta=0$ and the geometric and operating conditions of RDC. The result is a set of two quasi-linear ordinary differential equations, where the Mach number for the reactants and products $\left(M_{R}\right.$ and $\left.M_{P}\right)$, are the independent variables. The equations are solved in terms of the non-dimensionalized quantities of the flow, and the 
problem is closed by imposing a jump condition across the detonation wave. The problem is governed by five non-dimensional parameters that are functions of the operating conditions and the combustor geometry. Since not all of those parameters can be know beforehand, an optimization procedures needs to be performed in order to minimize the error between predicted Mach number and the Mach number determined by the jump condition. The necessary iterative process is relatively computationally expensive, and at the time this model was developed (1960) it signified a big computational effort.

The work presented in [Zhdan et al., 1990] is the first detailed explanation of the modeling strategy for RDC injection systems. As mentioned in the introduction, simplification of the injection system is often performed assuming Laval nozzle behavior under variable back pressure. The study by [Zhdan et al., 1990] reports the issue of total pressure loss across the injection system from the injection reservoir. It highlights the fact that the majority of the injection section operates at over expanded conditions. This represents an important factor for the present study, since the pressure loss across the injection system is to be minimized in order to maintain high thermodynamic efficiency of the cycle.

Numerical studies performed for RDC share many common factors, but this section highlights the differences encountered in some of the most relevant numerical studies. Numerical predictions of RDC are usually performed under the assumption of negligible influence of viscous terms [Hishida et al., 2009, Kindracki et al., 2011a, Liu et al., 2012, Schwer and Kailasanath, 2011, Shao et al., 2010, Yi et al., 2009, Zhdan et al., 2007]. Only a few of more recent studies [Frolov et al., 2013, Swiderski, 2013, Towery et al., 2014] have addressed the influence of viscous and turbulence effects on RDC performance. The current study is to provide some contribution into that ongoing discussion.

Multiple strategies for modeling the chemical reactions of fuel oxidizer mixture can be encountered in the available literature. Two stage kinetic models composed by the equation for reactants mass fraction and induction time is used in [Schwer and Kailasanath, 2011, Zhdan et al., 2007] for the modeling of $\mathrm{H}_{2}-$ Air combustion. A similar approach is followed by [Schwer and Kailasanath, 2013] for acetylene and propane-air RDC operations. The use of a uni-molecular reaction for $\mathrm{H}_{2}-$ Air detonation based on the model developed in [Ma et al., 2005] for PDE studies is widely employed. Results presented in [Kindracki et al., 2011a, Liu et al., 2012, Shao et al., 2010] show acceptable agreement with experimental data while using this model. The use of reduced reaction mechanisms considering detailed reversible chemical reaction is not widespread. Lim [2010] used a reduced reaction mechanism with 18 reversible reactions for hydrogen air RDC. However, the majority of the studies have chosen to reduce computational cost over the prediction of detailed of chemical reactions. As part of the present work, the influence of reduced mechanism are assessed by predictions using chemical mechanisms with different complexity levels. Emissions characteristics for RDC regime remain to be 
established, and neither computational nor experimental studies have focused on determining the pollutant concentration for an RDC regime. Pollutant concentrations $\left(\mathrm{CO}, \mathrm{CO}_{2}, \mathrm{NO} x\right)$ are to be determined as a contribution of the present study for laboratory scale RDC.

Experimental and computational studies for RDC have been limited to annular combustion chambers with constant cross sectional area or with expansion due to the conical shape of the inner walls. Additionally, RDC cases are restricted to geometries where the mean diameter of the chamber is considerably larger than that of the gap within the inner and the outer wall of the chamber. Although a 3D cylindrical computational domain is considered in multiple studies [Kindracki et al., 2011a, Liu et al., 2012] it is a common practice to neglect the influence of curvature effects in RDC performance, and to simplify the domain to an unwrapped 2D plane. Results obtained using the 2D simplification from [Hishida et al., 2009, Liu et al., 2012, Zhdan et al., 2007] show good agreement with experiments. At the same time, such studies were successful for the depiction of the governing flow structures in RDC.However, 3D effects on flow structure and stability regimes of continuous detonation are to be addressed. When a 2D simplification of the domain is performed vertical boundaries are set as translational periodic boundaries, as succesfully proven in [Hishida et al., 2009, Liu et al., 2012, Zhdan et al., 2007]. The effect of the annular gap on the detonation wave structure is also addressed in the present work.

Most of the experimental and numerical studies on RDC are focused on combustion chambers in which the outer diameters range from $100-150 \mathrm{~mm}$ and inner diameters from $75-$ $130 \mathrm{~mm}$. However, [Kindracki et al., 2011a] performed numerical experimentation obtaining the adequate conditions to sustain continuous detonation for annular chambers of up to $380 \mathrm{~mm}$ of mean diameter. The literature review shows that the majority of studies on RDC have focused on laboratory scale combustion chamber characterization. The study of RDC for annular combustor at scales relevant to stationary power generation gas turbine remains to be performed.

Due to the geometrical simplicity of the computational domain, the use of an orthogonal uniform computational mesh is common. Average computational cell sizes range between $0.1 \mathrm{~mm}$ and 0.5mm [Hishida et al., 2009, Schwer and Kailasanath, 2011, Shao et al., 2010]. Those mesh sizes equate to ${ }^{1} / 2 \Delta_{I}$ and $2.5 \Delta_{I}$ for For $H_{2}-$ Air mixtures. Such level of spatial discretization is not expected to resolve all the characteristic structures of the detonation wave described in Section 2.1; however it has been proven that overall parameters such as $U_{D W}, P_{C J}$ and $T_{C J}$ are appropriatelly predicted. Details on this issue are covered in Chapter 4 of the present study. Additionally, adpative mesh refinement (AMR) has been adopted in the numerical study [Swiderski, 2013, Yi et al., 2009] of RDC. This methodology reduces the computational time, while maintaining an appropriate mesh resolution near the detonation front. Because of the intricate interaction of the flow and multiple flow discontinuities in RDC, the selection of the refinement region and parameter is not a straight forward task. 
It is a common practice in numerical simulation of RDC to eliminate the injection section from the computational domain and to replace it by the implementation of an injection model at the inlet boundary condition. This model assumes that the injection of the reactants is governed by the behavior of ideal Laval micro nozzle with varying back pressures. Details of the model are described in Chapter 3. The use of this model has shown good agreement with experimental studies [Zhdan et al., 2007]. However, recent studies performed by [Schwer and Kailasanath, 2012] have proven that by considering the injector mixture plenum, injector plate and the micro injector geometry into the computational domain, the range of conditions under which sustained continuous detonation can be achieved is reduced. The present study illustrates numerical simulations at elevated pressure; and results considering the modeled injection section and 3D injection section are compared. A hybrid approach to the inlet boundary condition modeling can be found in the work of [Hishida et al., 2009], where the oxidizer and fuel streams are injected at different locations of the domain. However the injection properties for both streams are calculated according to the Laval nozzle model. Predicted stability ranges for these simulations were also reduced relative to those of the simpler single laval nozzle injection model.

General treatment of outlet conditions for RDC simulations are based on supersonic exhaust of the detonation products [Kindracki et al., 2011a, Liu et al., 2012, Zhdan et al., 2007]. The aforementioned scenario correlates appropriately with the majority of experimental studies performed on RDC research, where combustion products are dumped into a reservoir tank at low pressure. Few studies have been carried out for increased values of outlet pressure [Schwer and Kailasanath, 2011, 2012]. The methodology employed by [Schwer and Kailasanath, 2011, 2012] focused on extending the computational domain close to the outlet of the combustion chamber. False diffusivity is increased within the extended region and skewed cells in the axial directions are used in order to reduce the reflection of the pressure waves from the outlet boundary conditions. Such situation is of great interest for the proposed study; however, it is believed that a non-reflective boundary formulation should be developed in order to completely eliminate the possibility of reflected pressure waves. The implementation of a partially non-reflective boundary condition $(N R B C)$ is described in the present study, and details on the implementation for RDC simualtions can be found in Chapter 5 .

Appropriate numerical simulations of RDC are characterized by the capability of capturing the flow structures developed in the combustion chamber. The temperature contour plots presented by [Schwer and Kailasanath, 2011] and reproduced in Figure 2.8 are representative of the flow structures obtained for RDC numerical simulations. Such result corresponds to the numerical predictions for an annular chamber with an inner and outer diameter of $130 \mathrm{~mm}$ and $150 \mathrm{~mm}$ respectively. Figure 2.8 can be used to provide additional explanation of the flow structures developed in RDC, as a supplement to the explanation given in Chapter 1. The detonation 
front $A$ travels in the azimuthal direction towards the fresh mixture region $G$. An oblique shockwave develops due to the interaction between the detonation wave and the combustion products from the previous cycle. Flow instabilities on the slip surface $C$ can be observed for certain operation conditions, and they can also be present in the combustion front $E$ formed between the combustion region and the detonation products [Hishida et al., 2009]. An oblique shock wave $B$ is formed along the azimuthal direction of the combustion chamber, as it interacts with and is reflected from the combustion front $E$. A secondary contact surface $D$ is formed between the detonation products immediately behind the detonation front and those behind the oblique shock wave. The strength of this wave is highly dependent on the outlet pressure of the combustion chamber. Due to the high pressure immediately behind the detonation wave $A$, the inlet region $F$ is blocked. As the detonation products expand behind the detonation wave, injection of reactants into the domain is resumed and are either consumed by the combustion front or the detonation wave.

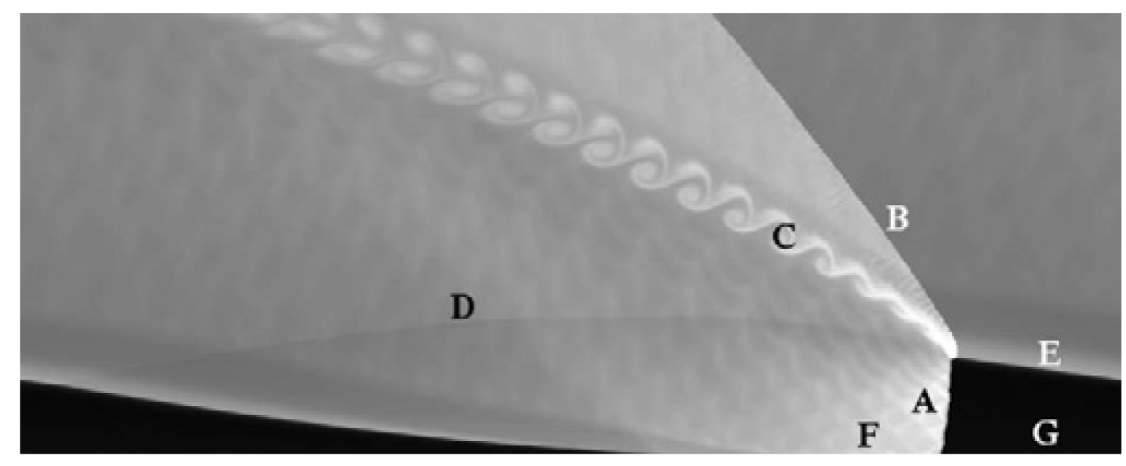

FIGURE 2.8: 2D temperature contour for RDC simulation, from [Schwer and Kailasanath, 2011]

The unsteady nature of RDC flows is usually reported by pressure history at given locations of the computational domain. When experimental data for pressure history is available quantitative comparison is possible. Calculation of average detonation wave velocity can be performed based on the time elapsed between the passage of the detonation wave.

The results presented by [Schwer and Kailasanath, 2011] are of great relevance to the present study. [Schwer and Kailasanath, 2011] analyzes the influence of modifying the ratio of stagnation injection pressure $\left(P_{o, m}\right)$ over the outlet pressure $\left(P_{\infty}\right)$ in RDC performance. Two parametric studies were performed for a cylindrical chamber of $D_{\text {in }}=130 \mathrm{~mm}$ and $D_{\text {out }}=$ $150 \mathrm{~mm}$, operating under the injection of $\mathrm{H}_{2}-$ Air. The first study focused on increasing the outlet pressure $P_{\infty}$ while maintaining a constant stagnation injection pressure $P_{o, m}$. The second study, varied $P_{o, m}$ while maintaining $P_{\infty}=1 \mathrm{~atm}$. For both cases, values of $\frac{P_{\infty}}{P_{o, m}}$ equal to 2.5 , $3,4,6,10,12,16$ and 21 were simulated. In their work it was presented that there is a critical pressure ratio $\frac{P_{\infty}}{P_{o, m}} \approx 4$ at which the detonation front is drastically influenced by the outlet pressure. Similarly, performance parameters for RDC, such as specific impulse, are reported to steadily decrease as the outlet pressure increases [Schwer and Kailasanath, 2011]. It is worth 
mentioning that the limiting value of $P_{\infty}$ depends on the combustion chamber and injection geometry, and the injection conditions. The influence of the injection section configuration on the limiting value of $P_{\infty}$ is adressed in the current study.

The numerical studies presented in this section of the literature review evidence the growing interest of using CFD as a predicting tool for RDC performance. Due to the fact that the major interest of RDC has been for propulsive application, most of the computational studies have considered operational regimes for low pressure outlets. Since only a few research studies have been made for RDC under increased outlet pressures [Schwer and Kailasanath, 2011], the present investigation proposes the development of a computational strategy that can be used to adequately predict RDC performance under increased pressure conditions relevant to gas turbine applications. It is believed that this will allow narrowing of the knowledge gap leading to a reliable feasibility study of RDC for gas turbine applications.

\subsection{Conclusions from Literature Review}

After analysis on the subject of RDC the following conclusions can be made:

- Although most of the numerical and experimental studies for RDC have focused on its performance for propulsive application, recent research efforts evidence the importance of obtaining a better understanding of RDC at elevated operating pressures.

- The nature of detonation combustion makes RDC a suitable option to obtain pressure gain across the combustor. However, in order to reach industrial application of detonation combustion in GT, several technical difficulties remain to be addressed. For example its performance under elevated pressures needs further examination.

- The presence of a wide range of temporal and spatial scales requires the appropriate selection of models and solution strategies in order to guarantee low computational cost. 


\section{Chapter 3}

\section{Model Considerations}

As mentioned in Chapter 1 and Chapter 2, the flow developed in rotating detonation combustion is highly complex. The compressible reactive nature of detonation phenomena and the intricate interactions among the flow discontinuities lead to stringent requirements on the numerical strategy to simulate this problem.

The present chapter covers the most relevant aspects of the selection of a numerical model and the development of a solution strategy for the study of RDC at operating coditions relevant to GT applications.

First, the governing equations necessary to model the problem of RDC are described, the terms that can be simplified under certain assumptions are mentioned, and the treatment of chemical reactions is covered. Then, the characteristics of the CFD solver used during the present study are provided, and the different possible configurations of the solver are stated. Following that, a description of the solver is provided together with the explanation of: the computational domain, the different degrees of domain simplification, and the partition of the domain into a computational mesh. Additionally, a description of the formulation used for the boundary conditions is provided. At last, a description on the different initial conditions used to initialize a one directional detonation wave is presented.

\subsection{Governing Equations}

Numerical simulations of RDC are often performed under the assumption of negligible viscous or diffusive effects, reducing the governing equations to either 2D or 3D Euler equations for unsteady compressible reactive flows. On the other hand as part of this study, the effects of viscous and diffusive terms on RDC simulations are assessed. Eventually, the set of governing equations 
presented in this section corresponds to the three dimensional Navier-Stokes equations. Equation 3.1 presents the in integral vector form for an arbitrary control volume of compressible reactive flow.

$$
\frac{\partial}{\partial t} \int_{V} \vec{W} d x+\oint[\vec{F}-\vec{G}] d A=\int S d V
$$

where,

$$
\vec{W}=\left[\begin{array}{c}
\rho Y_{1} \\
\vdots \\
\rho Y_{k} \\
\vdots \\
\rho Y_{N S} \\
\vdots \\
\rho \\
\rho u_{x} \\
\rho u_{y} \\
\rho u_{z} \\
\rho E
\end{array}\right], \vec{F}=\left[\begin{array}{c}
\rho Y_{1} \vec{v} \\
\vdots \\
\rho Y_{k} \vec{v} \\
\vdots \\
\rho Y_{N S} \vec{v} \\
\vdots \\
\rho \vec{v} \\
\rho u_{x} \vec{v} \\
\rho u_{y} \vec{v} \\
\rho u_{z} \vec{v} \\
\rho E \vec{v}
\end{array}\right], \vec{G}=\left[\begin{array}{c}
D_{1} \nabla\left(\rho Y_{1}\right) \\
\vdots \\
D_{i} \nabla\left(\rho Y_{k}\right) \\
\vdots \\
D_{N z} \nabla\left(\rho Y_{N s}\right) \\
\vdots \\
0 \\
\tau_{x i} \\
\tau_{y i} \\
\tau_{z i} \\
\tau_{i j}+q
\end{array}\right], \& \vec{G}=\left[\begin{array}{c}
\dot{\omega}_{1} \\
\vdots \\
\dot{\omega}_{k} \\
\vdots \\
\dot{\omega}_{N S} \\
\vdots \\
0 \\
0 \\
0 \\
0 \\
0
\end{array}\right]
$$

When diffusive effects are neglected, the diffusive term vector $(\vec{G})$ is equal to zero yielding the Euler equations for compressible and reactive flows. For this analysis, the ideal gas equation is used as equation of state. This model is defined as given in Equation 3.3 and Equation 3.4. This is a commonly implemented formulation [Yi et al., 2009, Zhdan et al., 2007].

$$
\begin{gathered}
\rho=\sum_{k=1}^{N_{s}} \rho_{k} \text { with } \rho_{k}=\rho Y_{k} \\
P=\sum_{k=1}^{N_{s}} \rho_{k} \frac{\bar{R}}{M w_{k}}
\end{gathered}
$$

The total energy $(E)$ is defined in Equation 3.5.

$$
E=\int_{T_{r} e f}^{T} C_{p, m i x} d T+\sum_{k=1}^{N_{s}} Y_{k} h_{f}^{o} T_{r e f}+\frac{\|\vec{v}\|^{2}}{2}-\frac{P}{\rho}
$$

Although the influence of turbulence on a confined detonation front has been explored through experimental and numerical studies by [Ingram et al., 1998], the effect of turbulence 
and turbulence modeling strategies on RDC prediction are not addressed in detail in the available literature. In [Frolov et al., 2013] Unsteady Reynolds Averaged Navier Stokes (URANS) simulations of RDC were performed. Their study addressed the issue of thermal management of RDC for propulsive applications. Due to the lack of comparison between inviscid and turbulent calculations in that study, a direct assessment of influence of turbulence on model predictions was not possible.

The modeling of chemical reactions during the present work is performed under two main strategies:

First, a uni-molecular reaction mechanism is used for preliminary calculations and parametric studies. This one step unimolecular reaction can be written as in Equation 3.6, where the forward reaction rate $\left(k_{f}\right)$ is calculated assuming Arrhenius form as given in Equation 3.7.

$$
\begin{gathered}
\mathrm{R} \stackrel{k_{f}}{\longrightarrow} \mathrm{P} \\
k_{f}=A_{r} e^{\left(-E_{r} / \bar{R} T\right)}
\end{gathered}
$$

The values for the pre-exponential factor $\left(A_{r}\right)$, the activation energy $\left(E_{r}\right)$, the enthalpy of reaction and thermodynamic properties were used as presented in [Ma et al., 2005]. These parameters were calibrated by [Ma et al., 2005] predict the CJ state for $\mathrm{H}_{2}$-Air detonations, and are equal to $A r=7.5 \times 10^{9} \mathrm{~s}^{-1} \& E_{r}=1.08 \times 10^{8} \frac{\mathrm{J}}{\mathrm{kmol}}$. Validation of this reaction model for the prediction of a single tube Pulse-Detonation-Engine is presented in [Ma et al., 2005]. The rate of consumption for the reactants is given by Equation 3.8, where $\left[C_{R}\right]$ is the molar concentration of the reactants. For the implementation of this chemical model it is assumed, as in [Ma et al., 2005], that specific heat ratio $(\gamma)$ is constant for the reactants and the products, and that it is equal to 1.29 . The heat release is also selected in order to appropriately predict the CJ conditions and is equal to $2.720 \times 10^{6} \frac{\mathrm{J}}{\mathrm{kg}}$.

$$
\dot{\omega}_{R}=-k_{f}\left[C_{R}\right]
$$

The second approach consists of implementing reduced chemical reaction mechanisms that model the chemistry relevant to pollutant concentrations of interest for gas turbine applications. The comparison of predictions achieved using different chemical reaction models was made to elucidate the influence of chemistry models on RDC simulations. The multi step mechanism used for $\mathrm{H}_{2}-\mathrm{Air}$ combustion is based on an integration of the $\mathrm{H}_{2}-\mathrm{O}_{2}$ oxidation mechanism developed by [Li et al., 2004], and the $N O_{x}$ chemistry from GRI 3.0 reaction mechanism [Smith et al., 1999]. The details of the coupled mechanisms are given in Appendix A. The 
resulting mechanism includes 14 species and 40 reversible reactions. Rates of formation for multi-reaction models are calculated based on Equation 3.9.

$$
\dot{\omega}_{k}=M w_{k} \sum_{r=1}^{N k}\left(v_{k, r}^{\prime}-v_{k, r}^{\prime}\right)\left(k_{f}^{r} \prod_{k=1}^{N_{s}}\left[C_{k, r}\right]^{\eta_{k, r}^{\prime}} k_{b}^{r} \prod_{k=1}^{N_{s}}\left[C_{k, r}\right]^{\eta_{k, r}^{\prime \prime}}\right)
$$

The thermodynamic properties of the chemical species are calculated using JANNAF polynomials [Chase and Force, 1998]. The transport properties for cases with uni-molecular reaction mechanisms are calculated using the kinetic theory of gases by [Hirschfelder et al., 1954], where the Lennard-Jones parameters for reactants and products are calculated as the molecular weighted average values based on the equilibrium compositions of the reactants and combustion products.

Simulations where diffusive effects are considered were performed under the assumption of turbulent or laminar flow. When laminar flow was considered, the stress tensor was calculated under the assumption of Newtonian fluid, and mass diffusion was calculated assuming Fick's Law of diffusion. For the simulations where turbulent effects were considered, the two-equation standard $\kappa-\epsilon$ model by [Launder and Spalding, 1974] was used. Under this model, the conservation equations for the turbulence kinetic energy $(\kappa)$ and the dissipation rate $(\epsilon)$ are solved. The turbulent viscosity $\left(\mu_{t}\right)$ is then calculated based on Equation 3.10, while the effective mass diffusion coefficient due to turbulent $\left(D_{t}\right)$ is calculated based on the turbulent Schimdt number $\left(S c_{t}\right)$ using Equation 3.11. The effect of the wall for turbulent flow simulations was taken into account using standard wall functions [Launder and Spalding, 1974]. The chemical reaction rates were calculated using Equation 3.8 and Equation 3.9, for the the simulations were turbulence effects are condsidered. The effect of turbulence on the reaction rates was neglected.

$$
\begin{gathered}
\mu_{t}=\rho C_{\mu} \frac{\kappa^{2}}{\epsilon} \\
S c_{t}=\frac{v_{t}}{\rho D_{t}}
\end{gathered}
$$

\subsection{Solver Details}

Numerical investigation of rotating detonation combustion has been mostly performed using inhouse Computational Fluid Dynamic solvers, mainly from academic or research institutions. To the best of the author's knowledge, and based on the literature review presented in Chapter 2, none of the previous numerical studies of RDC had been performed using the commercial CFD solver, ANSYS-Fluent. The work presented by the author and in [Escobar et al., 2013], is 
the first reported numerical study of RDC using the commercial CFD solver ANSYS-Fluent. The validation of this commercial CFD solver for RDC simulations consists of an important contribution to the engineering community. The CFD results presented in the following chapters were obtained using $A N S Y S^{\circledR}$ Academic Research, Release 14.5.

The linearization of the governing equation in ANSYS-Fluent ([Fluent, 2009]) is performed using the control volume approach. The solution strategy of the linearized equations depends on the type of solver selected. A total of three different of solvers are available in ANSYSFluent, which are: implicit density based solver (DBS), explicit density based solver (DBSE) and implicit pressure based solver (PBS). During the present study only the DBS and the PBS are addressed and utilized. As stated in the ANSYS-Fluent user's guide ([Fluent, 2009]), for the DBS, the governing equations (Equation 3.1) are solved in a coupled fashion, while PBS solves the governing equations in a segregated manner.

Multiple discretization schemes, gradient calculation methods, flux calculation strategies (for DBS only) and the pressure-velocity coupling method (for PBS only) are available in ANSYS ${ }^{\circledR}$ Academic Research, Release 14.5. Figure 3.1 shows an organigram of the different methodologies implemented during the present research. The results presented in Chapter 4 provide an evaluation of the DBS and PBS solver when calculating shock wave propagation of inert gases and detonation wave propagation in a $2 \mathrm{D}$ channel.

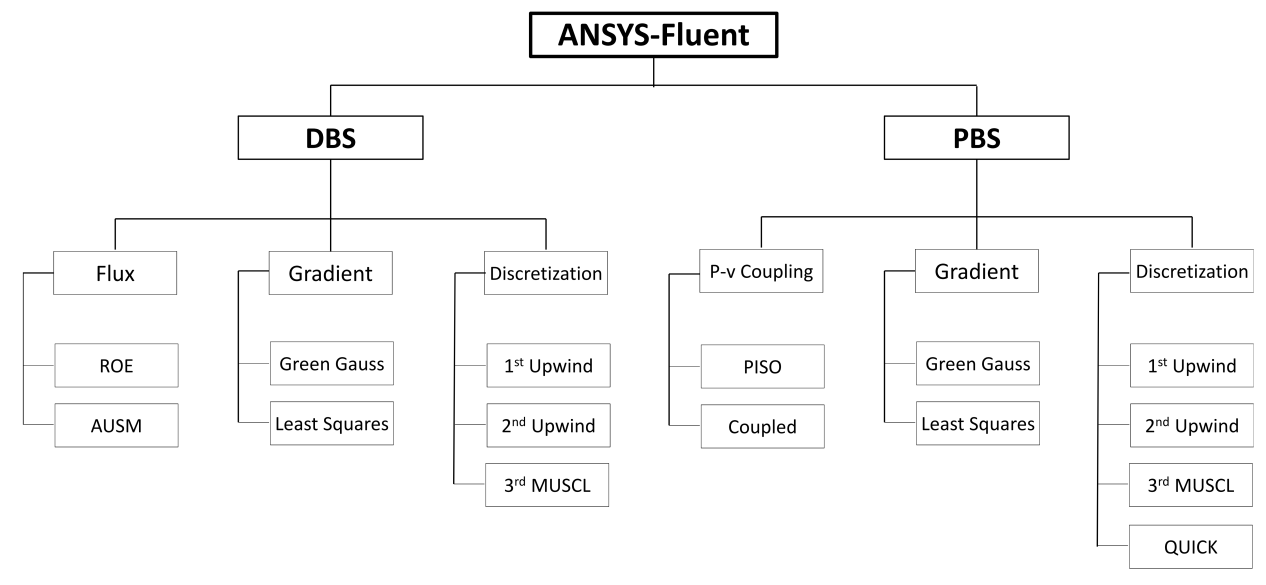

FIGURE 3.1: ANSYS Fluent DBS and PBS

\subsection{Computational Domain}

Due to the periodic nature of RDC, the geometric configuration of the combustion chamber is restricted to volumes of revolution. Although annular chambers are the most common geometry used for RDC, toroidal and conical combustion chambers have also been reported [Bykovskii et al., 2009]. Additionally, more complex geometries with expansion sections and coupling 
system with gas turbine stages were subject of numerical and experimental investigation by [Swiderski, 2013]. For the current study, the geometry of the combustion chamber is restricted to annular chambers. Such geometric configuration has advantages due to its simplicity and represents a better selection towards model validation due to the availability of experimental data. However, due to the flexibility of the computational model developed and tested as part of the present research effort can be implemented for the modeling of combustion chamber with complex geometrical set ups.

One of the main focuses of this investigation is the numerical investigation of RDC in annular chambers. The dimensions are based on laboratory scale combustors for which experimental data is available in the literature. The identified geometric parameters related to the combustion chamber are: the inner diameter $\left(D_{\text {in }}\right)$, the outer diameter $\left(D_{\text {out }}\right)$, and the combustion chamber length $\left(L_{c}\right)$. Table 3.1 presents a summary of the range of this geometric parameters covered in this present study.

TABLE 3.1: Geometric parameters for RDC in annular chamber

\begin{tabular}{ccc}
\hline \hline Parameter & Value & Units \\
\hline$D_{\text {in }}$ & $80-95$ & $\mathrm{~mm}$ \\
$D_{\text {in }}$ & $100-150$ & $\mathrm{~mm}$ \\
$L_{c}$ & $100-140$ & $\mathrm{~mm}$ \\
\hline
\end{tabular}

Additional to the configuration of the combustion chamber, the design of the injection sections plays a very important role in the performance of RDC. Although premixed injection of fuel and oxidizer was used in the early stages of RDC research [Voitsekhovsky, 1960], this set up is no longer used in experimental studies due to flashback hazard. The modern RDC injection systems have separated streams for the injection of fuel and oxidizer. The most common configuration of RDC injection system consists of an annular slot through which the oxidizer is injected into the combustion chamber. In this configuration the fuel is injected through a set of separated fuel injection holes, as observed in Figure 2.6. With this set up, the fuel and oxidizer mix near the oxidizer expansion plane. The fuel can be injected from the inner insert or from the head end of the combustion chamber. The geometrical parameters that govern the performance of the injection section include cross sectional area of the throat for the oxidizer annular gap $\left(A_{t}\right)$, the total area of fuel injection holes $\left(A_{f}\right)$ and the the cross sectional area of the expansion plane $\left(A_{e}\right)$ - for annular chamber this is equal to the cross sectional area of the combustion chamber. Additional to the geometrical parameters of the injection section, the stagnation pressure $\left(P_{o, m}\right)$ and the stagnation temperature $\left(T_{o, m}\right)$ for the injection of the reactants determine the performance of the injection section. These parameters together with the geometric parameters, determine the mass mass flow rate into the combustion chamber.

As mentioned previously and as described in Section 2, the geometry of experimental RDC combustors has a wide range of length scales and a moderate degree of complexity. 
In order to reduce the computational cost of RDC simulations, different domain simplification strategies have been widely adopted. One common simplification is to eliminate the injection section from the computational domain, which is instead modeled using the injection model developed by [Zhdan et al., 1990]. Descriptions and details of this model are provided in Section 3.4. The resulting computational domain encompasses the annular combustion chamber extending from the expansion plane of the injection section until the combustion chamber outlet. It is then assumed that at the expansion plane (inlet of the simplified domain), the oxidizer and fuel are perfectly mixed. Figure 3.2 (a) shows a schematic representation of this domain simplification process.

A second stage of domain simplification can be applied to the resulting annular chamber. It consists of "unwrapping" the annular chamber onto a 2D planar domain (see Figure 3.2(b)). This simplification is based on the fact that $\Delta_{c}<<D_{\text {mean }}$, therefore the curvature effects are often neglected. Additionally, the ideal injection model eliminates the three dimensional nature of the injection section. This simplification is performed under the assumption that the wall effects (viscous and curvature) can be neglected. The solution would be representative of the flow in the mid annular section of the combustion chamber. The resulting domain is a very simple rectangular domain, for which high mesh resolution can be used without incurring into a very high computational cost.

For the purposes of this study, numerical simulations were performed using the three types of domains, which are shown in Figure 3.2. The effects of the previously mentioned simplification strategy were evaluated and simulation recommendations were formulated.

Subsequently, the computational domain was discretized by an orthogonal mesh with quadrilateral and hexahedral elements for 2D and 3D geometries, respectively. Uniform computational cell distribution was implemented, and the cell size and time step selection were selected based on CFD simulations of a 2D detonation tube (see Chapter 4). Meshes with cell sizes of 500 $\mu \mathrm{m}, 250 \mu \mathrm{m}$ and $100 \mu \mathrm{m}$ were evaluated. These computational cell sizes correspond to $2.5 \Delta_{I}, 1.3 \Delta_{I}$ and $1 / 2 \Delta_{I}$ for $H_{2}-$ Air detonation. As mentioned previously this spatial resolution is not sufficient to capture the detailed structure of the detonation wave. Still it appropriately predicts the global parameters of the detonation wave inside the RDC combustion chamber (see Section 4). Figure 3.3 shows a schematic representation of the computational mesh used for 3D simulation with and without the injection section as well as for 2D simulations.

In addition to the orthogonal meshes presented on Figure 3.3, AMR was implemented for a few numerical simulations of detonation waves in 2D channels. As shown in Section 4, successful simulation of 2D detonation wave was obtained using this strategy. Yet, software instabilities were encountered once applied to the 2D and 3D simulations of RDC and could not be implemented. 


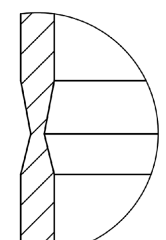

Injection Section

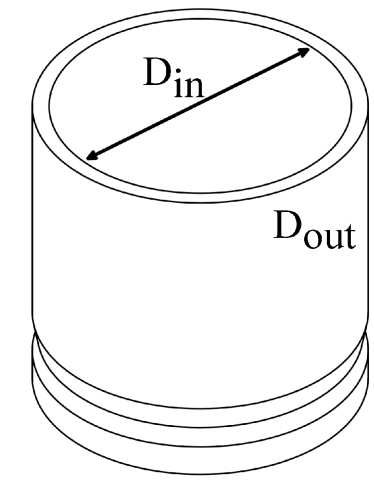

(a)

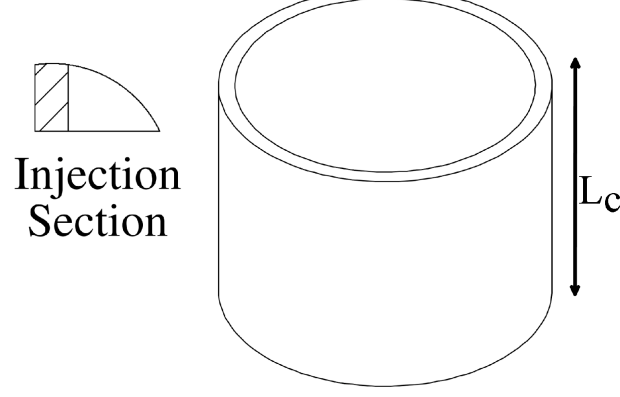

(b)

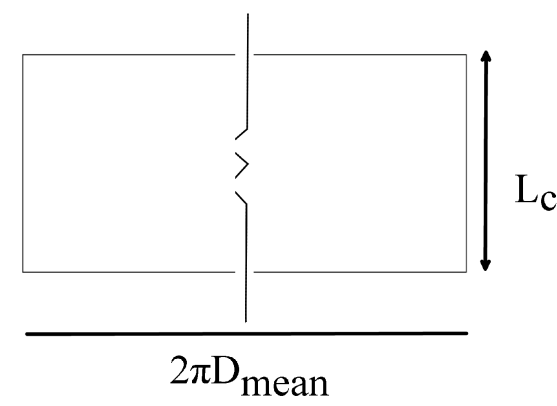

(c)

FIGURE 3.2: Computational domain simplification from 3D with injection system to 2D unwrapped domain. 3D domain with injection section (a), 3D with injection model (b), and 2D planar domain (b)

The wide range of temporal scales on RDC also requires the appropriate discretization of the problem in the temporal space. Despite the fact that implicit numerical schemes were always used, the time step $\left(\delta_{t}\right)$ was calculated in order to maintain the Courant number lower than 2. It is shown in Section 4.2, that CFL number larger than 2.0 lead to numerical instabilities in the prediction of the flow field behind the detonation wave. The Courant number (CFL) was calculated based on the $\mathrm{CJ}$ detonation wave velocity $\left(U_{C J}\right)$ and the cell size in the axial direction $(\delta x)$. Simulations where a multi-step mechanism was included were performed using the DBS solver in which the stiff chemistry solver developed by [Weiss, 1999] was implemented. This method operates under a time splitting principle and addresses the numerical stiffness caused by the chemical source terms in the species transport equation.

For the current study three different computational cluster were used: the cluster from the CFD\&AMP Center in WVU, the HPC Mountaineeer cluster and NETL's High Performance Computer for Energy and Environment. Since such resources were shared with multiple researchers and due to the limited quantities of parallel license for the commercial CFD solver ANSYS Fluent. Most of the simulations were performed using 20 processors distributed in 2 


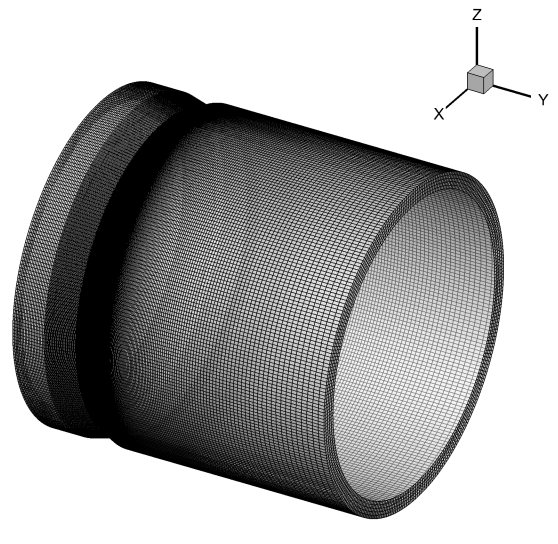

(a)

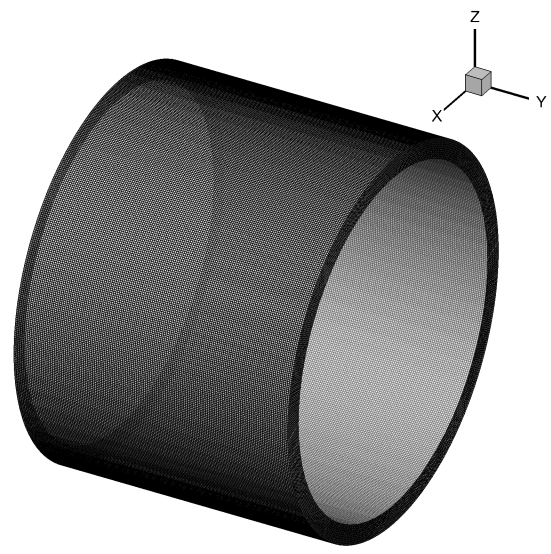

(b)

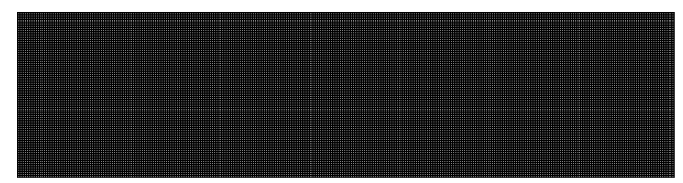

(c)

FIGURE 3.3: Computational mesh for 3D with injection section (a), simplified 3D domain (b) and 2D domain (c)

computer nodes with 10 processors on each node. Under this configuration a $2 \mathrm{D}$ simulation, using the single step chemical reaction model and a computational mesh of 2.2 million cells and

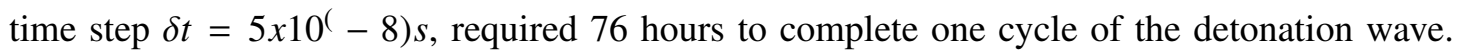
Where the averaged detonation wave period for the base case considered in the current study

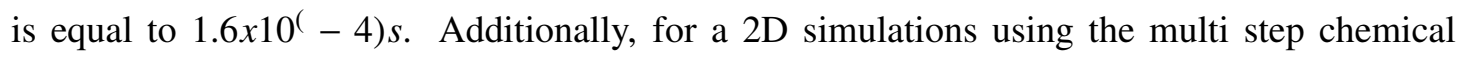
reaction model, with a computational mesh of 358 thousand computational cells and time step $\delta t=5 \times 10^{(-7)} s$ required 234 computational hours to calculate one cycle of the detonation wave.

\subsection{Boundary Conditions}

Appropriate formulation of the boundary conditions is required in order to obtain relevant results for the performance of RDC at elevated pressure conditions. In this section, the treatment of the inlet boundary condition depending on the degree of domain simplification is explained in detail. First, the treatment of the inlet boundary condition is explained, and the ideal injection model developed by [Zhdan et al., 1990] is covered (equations provided). Then, a description of the pressure outlet boundary condition is given, with focus on how to diminish pressure wave 
reflection from the outlet boundary. Additionally, wall treatment for 3D inviscid and viscous simulations is described.

Experimental studies on RDC often report the total mass flow rate of the reactants and/or the stagnation properties at the reactants injection manifold. The inlet boundary condition for computational simulations is determined based on the information of the operating condition $\left(P_{o, m}, T_{o, m} \& Y_{k, \text { inlet }}\right)$ and the geometry of the injection section $\left(A_{t} \& A_{e}\right)$. The inlet mass flow rate $\left(\dot{m}_{i n}\right)$ can be determined using Equation 3.12 ([Sutton and Biblarz, 2010]), while assuming choked condition in the injection section.

$$
\dot{m}_{\text {inlet }}^{*}=A_{t} P_{o, m} \gamma \frac{\sqrt{[2 /(\gamma+1)]^{(\gamma+1) /(\gamma-1)}}}{\sqrt{\gamma R T_{o, m}}}
$$

The implementation of the inlet boundary condition depends on the degree of domain simplification. If the injection section is included in the computational domain (Figure 3.3(a)), the inlet boundary condition is modeled as a pressure inlet. The pressure inlet boundary condition is then, defined by imposing the stagnation quantities for pressure and temperature $\left(P_{o, m}\right.$ and $\left.T_{o, m}\right)$. Additionally, the mass fractions of the species at the inlet $\left(Y_{k, \text { inlet }}\right)$ needs to be prescribed, and the direction of the inlet flow is set to be normal to the inlet boundary. Nevertheless, if the domain is simplified by eliminating the injection section, the ideal injection model developed by [Zhdan et al., 1990] should be implemented. This model is based on the premise that the injection system can be modeled as a set of Laval micro nozzles. Thus, the injection quantities can be calculated based on the local pressure inside the combustion chamber, which varies with position and time due to the traveling detonation wave inside the combustion chamber.

The ideal injection model calculates the injection state of the reactants $\left(P_{\text {inlet }}(\vec{x}, t)\right.$, $\left.\left\|\vec{v}_{\text {inlet }}(\vec{x}, t)\right\|\right), \rho_{\text {inlet }}(\vec{x}, t)$, and $\left.T_{\text {inlet }}(\vec{x}, t)\right)$ as a function of the local static pressure at the expansion plane of the combustion chamber $\left(P_{\exp }(\vec{x}, t)\right)$. If the local static pressure inside the combustion chamber $\left(P_{\exp }(\vec{x}, t)\right)$ is greater than the stagnation pressure at the injection manifold $\left(P_{o, m}\right)$, the model assumes that there is no inflow of reactants, and the injection state is calculated using Equation 3.13. Realistic phenomena under this condition leads to backflow of the detonation products into the inlet plenum chamber; however, the presence of backflow is not considered when this model is implemented.

$$
\begin{gathered}
\text { For } \boldsymbol{P}_{\boldsymbol{o}, \boldsymbol{m}} \leq \boldsymbol{P}_{\text {exp }}(\vec{x}, t) \\
P_{\text {inlet }}(\vec{x}, t)=P_{\text {exp }}(\vec{x}, t) \\
\left\|\vec{v}_{\text {inlet }}(\vec{x}, t)\right\|=0.0 \\
\rho_{\text {inlet }}(\vec{x}, t)=\rho_{o, m}\left[1-\left(\frac{\left\|\vec{v}_{\text {inlet }}(\vec{x}, t)\right\|}{U_{\max }}\right)^{2}\right]^{(\gamma /(\gamma-1))} ; \\
T_{\text {inlet }}(\vec{x}, t)=T_{\text {exp }}(\vec{x}, t)
\end{gathered}
$$


If the local pressure of the combustion chamber $\left(P_{\text {exp }}(\vec{x}, t)\right.$ is equal to or larger than the subsonic isentropic pressure $(P$ "), and smaller than the stagnation pressure at the injection manifold $\left(P_{o, m}\right)$, the flow is subsonic and isentropic. The injection state in this regime is calculated using Equation 3.14.

$$
\begin{gathered}
\text { For } \boldsymbol{P}^{\prime \prime} \leq \boldsymbol{P}_{\text {exp }}(\vec{x}, t)<\boldsymbol{P}_{\boldsymbol{o}, \boldsymbol{m}} \\
P_{\text {inlet }}(\vec{x}, t)=P_{\text {exp }}(\vec{x}, t) \\
\left\|\vec{v}_{\text {inlet }}(\vec{x}, t)\right\|=U_{\text {max }}\left[1-\left(\frac{P_{\text {inlet }}(\vec{x}, t)}{P_{o, m}}\right)\right] \\
\rho_{\text {inlet }}(\vec{x}, t)=\rho_{o, m}\left[1-\left(\frac{\left\|\vec{v}_{\text {inlet }}(\vec{x}, t)\right\|}{U_{\text {max }}}\right)^{2}\right]^{(\gamma /(\gamma-1))} ; \\
T_{\text {inlet }}(\vec{x}, t)=\frac{P_{\text {inlet }}(\vec{x}, t)}{\rho_{\text {inlet }}(\vec{x}, t) R_{\text {inlet }}}
\end{gathered}
$$

Nonetheless, if the chamber pressure resides between the isentropic pressures for subsonic and supersonic regime, $P$ " and $P^{\prime}$, non-isentropic injection of the reactants occurs. This condition corresponds to the state of over expanded nozzle; where shock waves are present in the expansion portion of the nozzle and losses in total pressure occur during this regime. The injection state is then calculated using Equation 3.15. Optimization of the injection system should consider operating the majority of the chamber under subsonic isentropic conditions or as close to this regime as possible. It is important to highlight that the injected velocity should still remain high enough to support continuous detonation.

$$
\begin{gathered}
\text { For } \boldsymbol{P}^{\prime} \leq \boldsymbol{P}_{\text {exp }}(\vec{x}, t)<\boldsymbol{P} \\
P_{\text {inlet }}(\vec{x}, t)=P_{\text {exp }}(\vec{x}, t) ; \\
\left\|\vec{v}_{\text {inlet }}(\vec{x}, t)\right\|=\frac{\sqrt{\left(\beta^{2}+4 U_{\text {max }}^{2}\right)}-\beta}{2} \\
\rho_{\text {inlet }}(\vec{x}, t)=\rho_{o, m}\left[1-\left(\frac{\left\|\vec{v}_{\text {inlet }}(\vec{x}, t)\right\|}{U_{\text {max }}}\right)^{2}\right]^{(\gamma /(\gamma-1))} ; \\
T_{\text {inlet }}(\vec{x}, t)=\frac{P_{\text {inlet }}(\vec{x}, t)}{\rho_{\text {inlet }}(\vec{x}, t) R_{\text {inlet }}}
\end{gathered}
$$

If the local pressure of the combustion chamber $\left(P_{\exp }(\vec{x}, t)\right.$ decreases to a value equal to or lower than the isentropic supersonic pressure $\left(P^{\prime}\right)$, the reactants are injected at supersonic velocities. The injection state at supersonic velocities is calculated using Equation 3.16. Supersonic injection is seldom encountered for RDC operations.

$$
\begin{gathered}
\text { For } \boldsymbol{P}_{\text {exp }}(\vec{x}, t) \leq \boldsymbol{P}^{\prime} \\
P_{\text {inlet }}(\vec{x}, t)=P_{\text {exp }}(\vec{x}, t) ; \\
\left\|\vec{v}_{\text {inlet }}(\vec{x}, t)\right\|=\frac{\sqrt{\left(\beta^{2}+4 U_{\text {max }}^{2}\right)}-\beta}{2} ; \\
\rho_{\text {inlet }}(\vec{x}, t)=\rho_{o, m}\left[1-\left(\frac{\left\|\vec{v}_{\text {inlet }}(\vec{x}, t)\right\|}{U_{\text {max }}}\right)^{2}\right]^{(\gamma /(\gamma-1))} ; \\
T_{\text {inlet }}(\vec{x}, t)=\frac{P_{\text {inlet }}(\vec{x}, t)}{\rho_{\text {inlet }}(\vec{x}, t) R_{\text {inlet }}}
\end{gathered}
$$


The pressures for isentropic subsonic and supersonic regimes for a Laval nozzle $(P$ ") and $\left(P^{\prime}\right)$, respectively, are calculated by solving the quadratic equation in Equation 3.17. Further, the injection factor $\beta$ is defined in Equation 3.18.

$$
\begin{gathered}
\frac{P}{P_{o, m}}\left[1-\left(\frac{P}{P_{o, m}}\right)^{\gamma-1 / \gamma}\right]^{1 / 2}=\left(\frac{2}{\gamma-1}\right)^{1 /(\gamma-1)}\left(\frac{\gamma-1}{\gamma+1}\right)^{1 / 2} \frac{A_{t}}{A_{e}} \\
\beta=\left(\frac{2 \gamma}{\gamma-1}\right)\left(\frac{P_{o, m}}{\dot{m}_{\text {inlet }}^{*} / A_{t}}\right)
\end{gathered}
$$

The implementation of the model into the boundary condition was carried out though imposition of the values of local static pressure at the inlet $\left(P_{\text {inlet }}(\vec{x}, t)\right)$, local static temperature at the inlet $\left(T_{\text {inlet }}(\vec{x}, t)\right)$, and local inlet velocity magnitude $\left.\left(\| \vec{v}_{\text {inlet }}(\vec{x}, t)\right) \|\right)$ at the inlet boundary. The direction of the flow is set to be normal to the inlet surface, and the species mass fraction at the inlet $\left(Y_{k, \text { inlet }}\right)$ are also specified. The injection model was incorporated into the commercial CFD solver ANSYS ${ }^{\circledR}$ Fluent Academic Research, Release 14.5. through a user defined function (UDF), where the $\mathrm{C}^{++}$subroutine was compiled and coupled into the solution process of the CFD problem. A section of the subroutine is given in Appendix B.

The appropriate implementation of the inlet boundary condition does not suffice to adequately define the numerical problem for RDC simulations. The outlet boundary condition also needs to be established, and it should be done according to the available experimental data. The majority of experimental RDC set ups discharge the combustion products into large volume reservoirs or large exhaust tanks. The information provided about that subsystem is often the volume of the tank and its initial pressure; the experimental set ups are designed such that the volume of the tank is large enough to avoid large temporal variations of the exhaust pressure during RDC operation. For the majority of the reported experimental data (see Section 2.3), exhaust pressures are below or very close to atmospheric pressure. Likewise, some experiments discharge the combustion products directly into the atmosphere, without having control over the exhaust conditions. This correlates with the fact that most of the research of RDC has focused on its propulsive applications (see Section 2.3). However, the experiments planned by NETL are to be performed for elevated outlet pressures; hence, the boundary condition formulation in the present study needs to appropriately model RDC under elevated outlet pressure conditions.

An adequate formulation for the outlet boundary condition at elevated outlet pressures is required. The implementation of the outlet boundary condition depends on the value of the exhaust or far-field pressure $\left(P_{\infty}\right)$. If $P_{\infty}$ is lower than the critical pressure for supersonic exhaust of the combustion products, then the Mach number at the outlet $\left(M_{\text {exit }}\right)$ will be equal or greater than 1. In such a case, there will be no influence of the far-field pressure on the domain, and the principal quantities at the outlet $\left(\vec{v}_{\text {exit }}, T_{\text {exit }}, P_{\text {exit }}, \rho_{\text {exit }}\right)$ are extrapolated from the flow in 
the interior of the domain. As a result, no information on the boundary condition is required [Ferziger and Perić, 2002]. Most of the numerical studies are performed under this regime; still these conditions do not fall into the main objective of this study. Given the fact that the exhaust pressures relevant for GT applications are greater than the critical exhaust pressure, subsonic velocities of the detonation products at the combustor outlet are expected. Due to the subsonic nature of the flow at the boundary, acoustic waves will reflect back into the domain from the boundary surface. This non-physical reflection of the acoustic waves is a well know numerical phenomena. It is also known that it often leads to decreased rates of convergence of the numerical solution, as mentioned by [Selle et al., 2004]. Additionally, these reflections may lead to inadequate coupling between the reaction fronts in the domain and the perturbations in the flow [Selle et al., 2004]. Accordingly, extensive research has been performed on the appropriate formulation of boundary conditions for compressible Euler and Navier Stokes (NS) equations [Bogey and Bailly, 2002, Selle et al., 2004, Torregrosa et al., 2012].

A common solution to the forementioned problem has been to develop a boundary condition formulation that can control the reflection of the waves whilst controlling the pressure of the mean flow. These types of boundary conditions are often referred to as non-reflective boundary conditions (NRBC). The NRBC formulation implemented in ANSYS ${ }^{\circledR}$ Academic Research, Release 14.5. is based on the work performed by [Rudy and Strikwerda, 1980, Selle et al., 2004]. The development of this formulation is based on the characteristic equations for laminar, one-dimensional, inviscid flows ([Selle et al., 2004]), where the amplitudes of the waves in the domain $\left(\mathcal{L}_{i}\right)$ are related to the primitive variables of the flow $\left(\vec{v}, T, P, \rho \& Y_{k}\right)$. For a subsonic outlet boundary condition, the main interest is given to the acoustic waves entering and leaving the domain through the outlet boundary $\mathcal{L}_{1}$ and $\mathcal{L}_{5}$, respectively. The formulation of an NRBC consists of calculating the value of $\mathcal{L}_{1}$ in order to control the pressure at the outlet boundary, while at the same time diminish the reflection of the acoustic waves that reach the outlet boundary. Three distinct possible scenarios can be formulated according to the control of $\mathcal{L}_{1}$ : First, if a constant pressure at the outlet boundary is desired, then it would be necessary to set $\mathcal{L}_{1}=\mathcal{L}_{5}$, making the amplitude of the incoming and leaving wave equal with opposite sign. In other words, all acoustic waves reaching the outlet are reflected into the domain which creates the before mentioned problems.

The second scenario consists of setting the amplitude of the incoming wave $\left(\mathcal{L}_{1}\right)$ equal to zero; this corresponds to a fully non reflective boundary condition. As stated by [Poinsot and Veynante, 2011] and proven in Chapter 5, a perfect NRBC leads to an "ill-defined" problem. Under this condition there will be no influence of the far field exhaust pressure $\left(P_{\infty}\right)$ on the boundary condition; the acoustic waves will not reflect on the boundary but there will be no control over the mean flow pressure. As shown in Chapter 5 , the lack of control on the outlet pressure leads to "drift" of the imposed $P_{\infty}$. The last situation consists on a partially NRBC, where the amplitude $\mathcal{L}_{1}$ is calculated in such manner that some of the acoustic waves do not 
reflect from the boundary condition, while others remain in order to maintain control over the mean flow pressure. The methodology implemented in ANSYS ${ }^{\circledR}$ Academic Research, Release 14.5. to calculate the value of $\mathcal{L}_{1}$ is called the linear relaxation method (LRM), that was developed by [Rudy and Strikwerda, 1980]. This method is based on calculating the value of $\mathcal{L}_{1}$ based on the local static pressure at the boundary $\left(P_{\text {exit }}(\vec{x}, t)\right)$ and the far field pressure $\left(P_{\infty}\right)$. The formulation for $\mathcal{L}_{1}$ is given in Equation 3.19.

$$
\mathcal{L}_{1}=K\left(P_{\text {exit }}(\vec{x}, t)-P_{\infty}\right)
$$

Here $K$ represents the restitution constant for the linear correction of the amplitude $\mathcal{L}_{\infty}$. The value of $K$ determines the cutoff frequency $\left(f_{c}\right)$, as given in Equation 3.20. The cutoff frequency sets the limit of the acoustic waves that will reflect or that will leave the computational domain once they reach the boundary condition. Frequencies above $f_{c}$ will not be reflected, while acoustic waves with frequencies lower than $f_{c}$ are, indeed, partially reflected. Ther partial reflection of the lower frequncy waves, carries the information of the imposed $P_{\infty}$ and avoid the problem to drift away from the desired value of $P_{\infty}$.

$$
f_{c}=\frac{K}{4 \pi}
$$

The calculation of $\mathrm{K}$ is then performed using equation Equation 3.21, based on the model of [Selle et al., 2004]. Where $\sigma$ is the relaxation coefficient, $M_{\text {mean }}$ is the mean Mach number of the flow, $c$ is the sound speed and $L_{r e f}$ is the reference length of the domain. The calculation of $\mathrm{K}$ in $A N S Y S^{\circledR}$ Academic Research, Release 14.5. differs slightly from Equation 3.21, where $M_{\text {mean }}$ is replaced by $M_{\max }$, the maximum mach number in the domain.

$$
K=\sigma\left(1-M_{\text {mean }}^{2}\right) \frac{c}{L_{r e f}}
$$

In the commercial CFD code $A N S Y{ }^{\circledR}$ Academic Research, Release 14.5., the only parameter that can be controlled for the NRBC model is the value of $\sigma$. As presented in the work of [Selle et al., 2004], the optimal value for $\sigma$ is in the order of $\pi$. Nontheless, as shown in Chapter 5, the selection of $\sigma$ for RDC simulations needs to based on the conditions of the problem. The application of NRBC with LRM for RDC simulations has not been reported in the literature. This study presents the strategy to select the appropriate parameters for the LRM in the prediction of RDC at elevated outlet pressure. The selection of $\sigma$, is based on minimizing the reflection of pressure waves reaching the outlet while maintaining appropiate control over the mean flow conditions. 
For numerical simulations of RDC using the 2D simplified domain (see Figure 3.3(c)), the vertical boundary conditions are set as translational periodic boundary conditions.

In addition to the inlet and outlet boundary conditions, it is necessary to establish the treatment of the wall boundaries for 3D RDC simulations. It is known that the the presence of walls leads to curvature, viscous and thermal effects. The influence of curvature manifests as gradients of pressure, temperature and density in the radial direction of the combustion chamber. It is expected that these effects become more significant as the ratio $\frac{\Delta_{I}}{D_{\text {mean }}}$ increases. In the present study, a set of 3D simulations are performed under the assumption of negligible diffusive effects. Under this assumption, compressible 3D Euler equations are solved and a slip condition at the wall is imposed. Therefore, neither wall shear nor heat flux from the fluid to the wall is exerted. On the other hand, if diffusive terms are considered the NS equation (Equation 3.1) are solved. In such cases, non-slip condition $\left(\left.\vec{v}(\vec{x}, t)\right|_{\text {wall }}=0\right)$ at the wall is imposed, and three different boundary conditions for the energy equation are considered. One condition is based on the the assumption of a perfectly insulated wall. In this case, the heat flux normal to the wall boundary is set to zero and a Neumann boundary condition is imposed as given in Equation 3.22. The boundary condition for adiabatic wall was selected in order to isolate the performance losses due to viscous friction only.

$$
\left.\frac{\partial T(\vec{x}, t)}{\partial \vec{n}_{\text {wall }}}\right|_{\text {wall }}=0
$$

Numerical simulations were also performed while considering non-adiabatic wall conditions. In such simulations, a mixed thermal boundary condition was implemented. A group of numerical simulations were performed using a thin wall model. Other set of simulations were performed while incorporating the solid region corresponding to the outer cylindrical wall of the combustion chamber into the computational domain. For both strategies natural convection and radiation into the environment was considered. The heat transfer coefficient on the outer surface of the combustion chamber $\left(h_{\text {conv }}\right)$ was calculated based on experimental correlations for natural convection of a horizontal cylinder.

\subsection{Initial Condition}

The initiation strategy is a crucial aspect for experimental and numerical studies of Rotating Detonation Combustion. In spite of the fact that this combustion regime is unsteady by nature, a periodic steady state can be achieved during continuous detonation operation. Achieving this periodic state is a function of the initiation or initial condition. The initiation procedure -experimental and numerical- has the objective of guaranteeing the formation and sustainability of one or multiple unidirectional detonation waves. As mentioned in Section 2.3, modern 
experimental set ups achieve unidirectional detonation using an external initiation tube, which is placed tangential to the annular combustion chamber (see Figure 2.6). Still, the initiation of unidirectional detonation waves for numerical simulations can be achieved through different strategies. In the present study, two initiation methodologies were evaluated:

The first adopted initiation strategy is based on the partition of the computational domain into three distinct rectangular regions (hexahedral for 3D). A schematic representation of the domain partition for this approach is given in Figure 3.4. The initial condition on each region is prescribed in order to facilitate the generation of a unidirectional detonation wave. Therefore, Region I is initialized with a non reactive mixture at low temperature, Region II with a reactive mixture at low temperature and low pressure, and Region III is patched with a high pressure high temperature mixture of combustion products. The specific values for each of the regions depend on the reaction mechanism used and the operating conditions considered. Values for RDC initiation for stoichiometric $\mathrm{H}_{2}-$ Air are shown in Table 3.2.

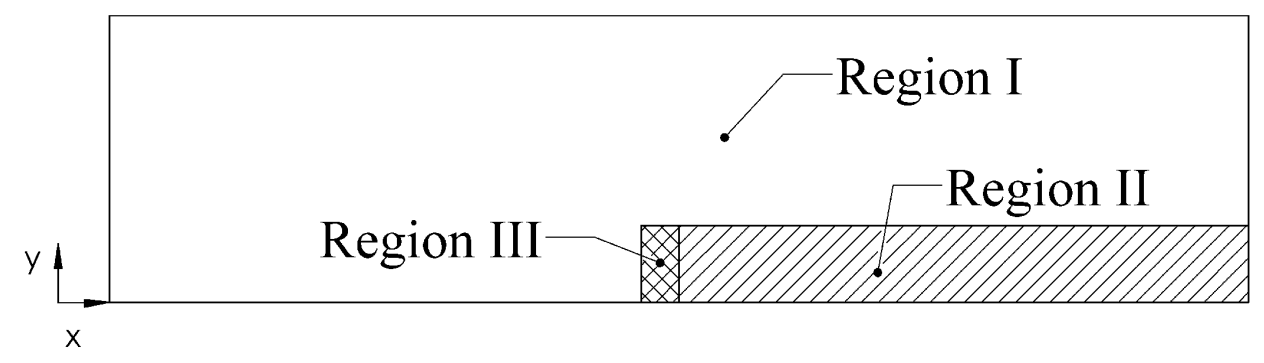

FIGURE 3.4: Simple initiation strategy for 2D domain

Although the schematic representation in Figure 3.4 is designed for the initial condition in a $2 \mathrm{D}$ domain, this strategy is also applicable for $3 \mathrm{D}$ simulations. Such initial condition is simple to apply; however, it leads to long transient behavior before the system reaches periodic steady state. Additionally, for cases where the exhaust pressure is close to the limiting pressure this initiation strategy does not lead to continuous detonation.

TABLE 3.2: Initial conditions for 2D RDC simulations

\begin{tabular}{ccccc}
\hline \hline Region & $\mathrm{T}[\mathrm{K}]$ & $\mathrm{P}[\mathrm{atm}]$ & $\begin{array}{c}\text { One Step Rxn. Mech. } \\
\text { Composition }\end{array}$ & $\begin{array}{c}\text { Multiple Step Rxn. Mech. } \\
\text { Composition }\end{array}$ \\
\hline I & 300 & 1 & $100 \%$ Products & $2 H_{2}+N_{2}$ \\
II & 300 & 1 & $100 \%$ Reactants & $2 H_{2}+\left(O_{2}+3.76 N_{2}\right)$ \\
III & $T_{C J}$ & $P_{C J}$ & $100 \%$ Products & $Y_{k, C J}$ \\
\hline
\end{tabular}

The second strategy includes a more sophisticated initial condition. It was developed and implemented in order to reduce the transients after the initial condition, and to increase the possibility of reaching continuous detonation at elevated exhaust pressure. It consists of the application of the 1D theoretical model developed by [Nicholls and Cullen, 1964] to calculate 
an initial condition favorable for obtaining continuous detonation. In this process, the 1D model was coupled with the injection model described in Section 2.4. As a result, a model capable of predicting the distribution of pressure, temperature and species for 2D and 3D domains was obtained. Due to the proximity of the calculated field to the actual solution of RDC simulations, the duration of transients was reduced and the exhaust pressure at which continues detonation can be achieved was extended. Details on the coupling process of the 1D analytical model and the injection model by [Zhdan et al., 1990] are provided in Chapter 5. Figure 3.5 shows the contour plots of the initial condition for 2D RDC simulations calculated using the coupled 1D model with the injection model. For this particular case, the exhaust pressure $\left(P_{\infty}\right)$ is equal to $1 \mathrm{~atm}$, and the reactants mixture corresponds to stoichiometric $\mathrm{H}_{2}+$ Air. The coupling of the 1D with the injection model and its implementation as predictive tool and initial condition is unique to the current study. The most advantageous implementation of such strategy was encounterd for RDC simulations at elevated outlet pressure. Such results are presented in Chapter 5

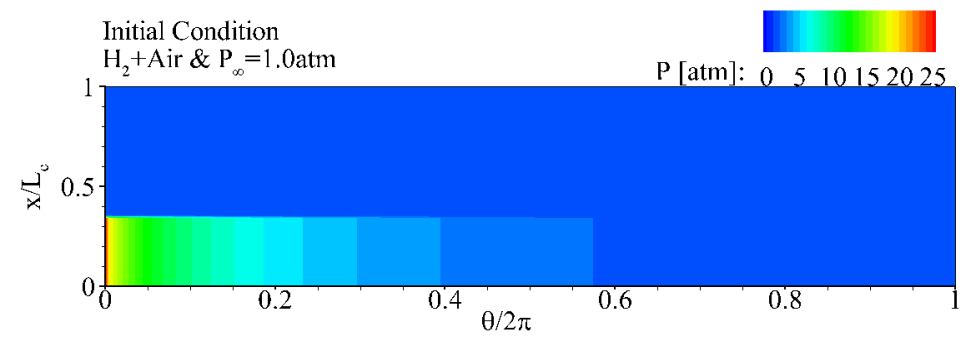

(a)

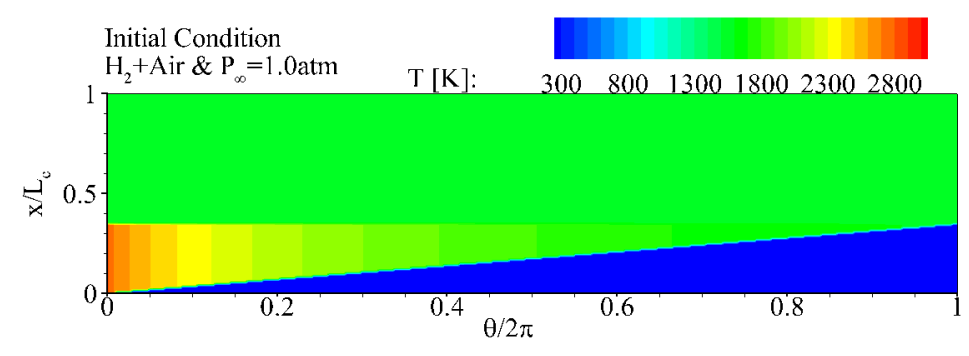

(b)

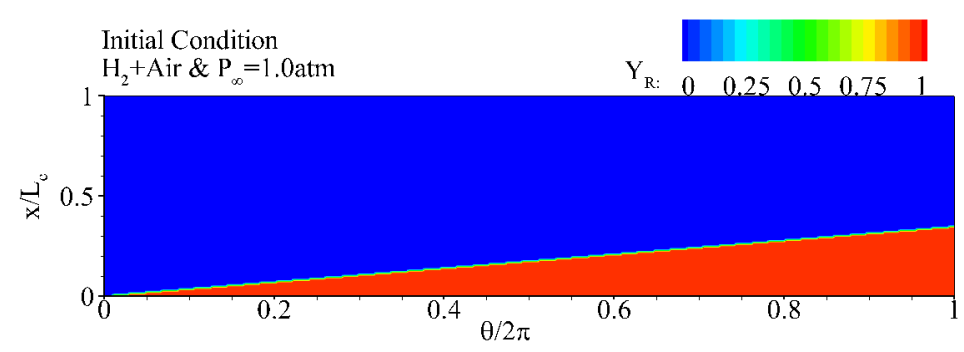

(c)

FIGURE 3.5: Initial condition for 2D RDC simulation from 1D model. Pressure (a), temperature (b) and $Y_{R}$ (c) contours. 


\subsection{Conclusions from Model Considerations}

- The governing equations necessary to describe the main physical phenomenon in RDC were selected. The fluid flow is to be modeled by the solution of the 2D and 3D Euler and Navier-Stokes equation, depending on the assumptions made for the diffusive terms. When diffusive effects are considered, laminar and turbulent simulations taking into account the diffusive transport of mass, momentum and heat were performed. The effects of turbulence on the mean flow in RDC were modeled using unsteady Reynolds Averaged Navier Stokes equations (URANS) with the 2-equations turbulence model $\kappa-\epsilon$.

- Two different strategies for the modeling of chemical reactions were selected: a 1 step unimolecular reaction model and a multi-step reduced chemical mechanism.

- The different degrees of domain simplification used in the current study were presented and the corresponding computational mesh structure was described.

- The model considerations for the treatment of the boundary conditions was given. The description of the wall model for the different assumptions on the transfer of momentum and heat was presented. Special attention was given to the formulation of the injection condition for the simplified domain simulations, and to the NRBC implemented at the outlet boundary.

- The two implemented initiation strategies were described. The presented schematics described the temperature, pressure and species distribution for the initial conditions. 


\section{Chapter 4}

\section{Model Validation}

In the present chapter, the developed model for RDC simulation are evaluated. These tests are presented in a way that the level of complexity for the validation cases gradually increases. First, the capabilities of the selected model and CFD solver to simulate compressible non-reactive flows are evaluated. The second validation case consists of the numerical simulation of the reactive flow of a detonation wave propagating in a 2D channel. The last validation case consists of the numerical prediction of Rotating Detonation Combustion for a laboratory scale experimental set up under supersonic exhaust conditions. The numerical results for the validation cases were compared with theoretical and experimental data, based on their availability. The quantification of the numerical error is presented and conclusions on their origins are drawn.

\subsection{Non Reactive Shock Tube Simulations}

In this section the capability of the selected solver to appropriately predict the flow for the nonreactive shock tube problem is evaluated.

Although the prediction of the flow in a $2 \mathrm{D}$ non-reactive shock tube is a relatively simple problem, it can serve the purpose of evaluating the capability of a CFD code to appropriately predict flow discontinuities in compressible problems. Additionally, the existence of a theoretical solution permits assessment of the numerical errors in a very precise fashion. Therefore, this validation case is used to evaluate the performance of multiple solver configuration and multiple mesh refinement levels. These results give an initial evaluation towards the prediction of the detonation phenomena and the discontinuities that occur during RDC.

The non-reactive shock tube problem has been widely studied, and it represents a valuable validation tool used by many authors in the CFD field. The formulation of the problem is often credited to [Sod, 1978]. Figure 4.1(a) illustrates the schematic representation of the 
problem, where a rectangular domain with length of $1 \mathrm{~m}$ and close ends is divided into two sections: the driver and the driven section. The driver section has a length of $0.5 \mathrm{~m}$ and is initially at pressure $\left(P_{4}\right)$ of $1.0 \times 10^{5} \mathrm{~Pa}$ and density $\left(\rho_{4}\right)$ of $0.116^{\mathrm{kg}} / \mathrm{m}^{3}$. The driven section corresponds to the remaining area of the domain, and it is initially at the driven pressure $\left(P_{1}\right)$ of $1.0 \times 10^{4} \mathrm{~Pa}$ and driven density, $\left(\rho_{4}\right)$ of $1.0^{\mathrm{kg}} / \mathrm{m}^{3}$. A diaphragm separates both regions at the initial condition $(t=0 s)$. For the present study, the gas is considered to be calorically perfect with a constant specific heat ratio $(\gamma)$ of 1.4 .

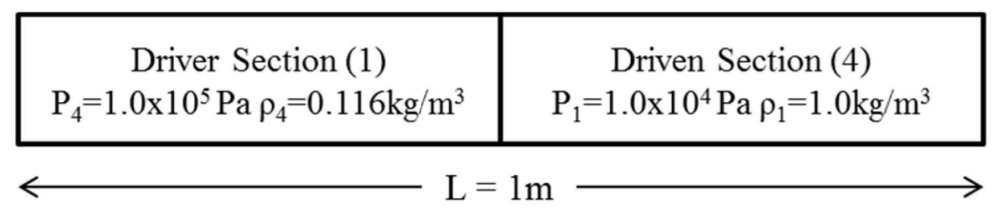

(a)

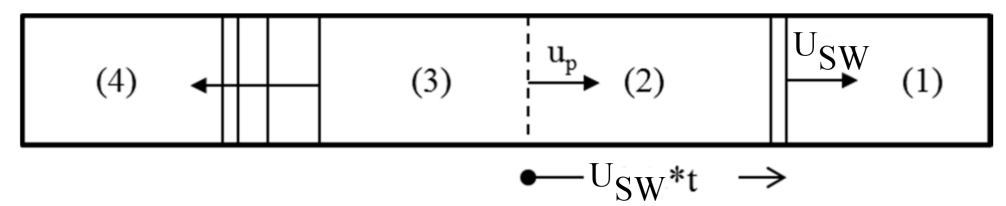

(b)

FIGURE 4.1: Schematic description of the non reactive shock tube problem, initial condition (a), flow structure at time $\mathrm{t}(\mathrm{b})$

Once the diaphragm is removed, a normal shock wave propagates from the driver section into the driven section. Additionally, an expansion wave propagates in the opposite direction into the driver section at a wave velocity $\left(U_{S W}\right)$. A contact surface is formed between the driver and the driven section, traveling at the particle velocity $\left(U_{p}\right)$. Figure $4.1(\mathrm{~b})$ exemplifies the regions and the schematic representation of the shock tube flow after the diaphragm is removed.

As shown in Figure 4.1(b), two additional regions are formed after the diaphragm is removed. The region 3 represents the part of the domain influenced by the presence of the expansion wave, whilst region 2 is influenced by the traveling shock wave. Regions 3 and 2 are separated by the presence of the contact surface.

The simulations performed for this validation study, are calculated by solving the two dimensional compressible Euler's equations; where the effects of viscous terms are neglected. The walls are considered to be iso-thermal and a slip wall condition is assumed. As mentioned in Section 3, the available density and pressure based solver (DBS and PBS) are evaluated.

For the simulations performed using DBS, two different schemes for the convective fluxes were evaluated: Roe flux-difference splitting scheme (ROE), and advection upstream splitting method (AUSM). In addition, two different methods for gradient calculations were assessed: the Green-Gauss cell based (GC) method, and the least square cell based gradient 
evaluation (SQC) method. Moreover, three different spatial discretization schemes were tested: first order upwind (1UP), second order upwind (2UP), and third order MUSCL (3M).

Numerical simulations were also performed using the the pressure based solver (PBS). In this set of simulations two different pressure-velocity coupling schemes were tested: the segregated algorithm for pressure velocity coupling, Pressure-Implicit with Splitting of Operators (PISO) and the coupled approach, where the momentum and the pressure-based continuity equation are solved simultaneously [Fluent, 2009]. Additional to the spatial discretization schemes mentioned for the density based solver, the QUICK (Q) scheme was also tested while using the pressure based solver. The methods evaluated for gradient calculations for the pressure based solver were the same as those tested for the density based solver. A summary of the discretization schemes, flux and gradient calculation methods, and the pressure velocity algorithms studied has given in Figure 3.1.

The computational domain was divided using orthogonal quadrilateral mesh elements, where four mesh resolutions were tested. The sizes of the computational cells in the axial $(\delta x)$ and transverse direction $(\delta y)$ for the different mesh resolutions are given in Table 4.1. The time step $(\delta t)$ was adjusted according to the mesh resolution in order to maintain a CourantFriederich-Lewis (CFL) criteria almost constant. The CFL number was calculated based on the theoretical value of the shock wave velocity. The CFL number used for the simulation of non reactive shocktube problem was approximately 0.2 , whereas the CFL number for the reactive simulations was approximately 2 .

TABLE 4.1: Mesh and time step characteristics for non reactive shock tube problem

\begin{tabular}{ccccc}
\hline \hline Mesh & $\delta x[\mathrm{~mm}]$ & $\delta y[\mathrm{~mm}]$ & $\delta t[\mathrm{~s}]$ & $C F L$ \\
\hline M1 & 10 & 10 & $3.5 \times 10^{-6}$ & 0.20 \\
M2 & 4 & 5 & $1.25 \times 10^{-6}$ & 0.18 \\
M3 & 2 & 2.5 & $6.25 \times 10^{-7}$ & 0.18 \\
M4 & 1 & 1.25 & $3.125 \times 10^{-7}$ & 0.18 \\
\hline
\end{tabular}

\subsubsection{Results using the Density Based Solver (DBS)}

In order to establish the influence of flux type calculation and discretization scheme while using DBS, a total of twelve simulations were performed. This stems from: two types of flux calculation methodologies, two gradient calculation strategies, and three spatial discretization schemes. The predicted shock wave velocity for these twelve simulations can be found in Appendix C (Table C.1).

The overall behavior of the shock tube problem is replicated in all of the twelve cases studied, and the propagation of the shock wave and expansion wave are adequately predicted. 
The maximum relative error for the averaged shock wave velocity reports $6.4 \%$. Such maximum relative error was obtained for the simulations performed using ROE and the 1UP scheme. The remaining configurations led to a relative error for the averaged shock wave velocity equal to $2.4 \%$. Additional to the over prediction of the shock wave velocity, results using 1UP showed significantly more numerical diffusion near the shock and expansion wave fronts and they evidence the presence of numerical diffusion near the shock wave front. This is the result of the relatively coarse mesh $(\delta x=10 \mathrm{~mm})$. Figure 4.2 ( $\mathrm{a}$ and $\mathrm{b}$ ) show the pressure distribution along the axial direction at $t=4.375 \times 10^{-4} \mathrm{~s}$. These results were obtained using DBS-ROE-GC with three different spatial discretization schemes: 1UP, 2UP and 3M.

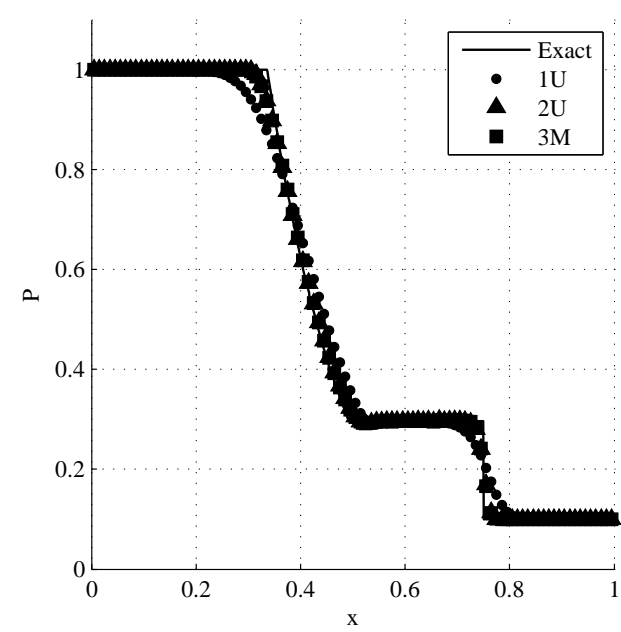

(a)

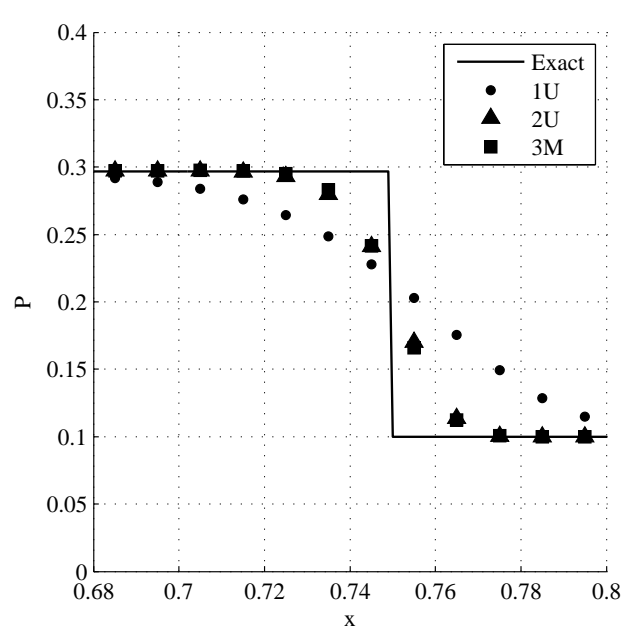

(b)

FIGURE 4.2: Pressure distribution for non reactive shock tube simulation DBSE-ROE-GC. Complete domain (a) and zoom on shock wave front (b)

Additionally, as expected the 1UP presents the most numerical diffusion of the spatial discretization schemes studied. On the other hand, little difference is observed for higher order schemes. Results obtained using AUSM flux schemes show similar behavior to those showed in Figure 4.2.

The influence of mesh resolution on the numerical prediction was studied for simulations using DBS, AUSM, SQC and 2UP. The pressure distribution for the three mesh resolutions are shown in Figure 4.3 and the predicted shock wave velocity is given in Table C.2. The shock wave resolution is significantly improved from M1 to M2, as shown in Figure 4.3(b). Additionally, the prediction of the shock wave velocity $\left(U_{S W}\right)$ was improved. It is observed that the relative error changed from $2.4 \%$ with $\mathrm{M} 1$ to $0.4 \%$ with $\mathrm{M} 2$. Numerical predictions with higher levels of spatial resolution (M3 and M4), led to relative errors lower than $0.3 \%$, where the shock wave velocity is always over predicted. It can be then concluded that for the numerical prediction of the non reactive shock tube problem with DBS, AUSM, SQC and 2UP, a computational cell size $(\delta x)$ equal or lower than $4 \mathrm{~mm}$ is to be used in order to obtain a relative error $<1 \%$ with respect to the shock wave velocity. 


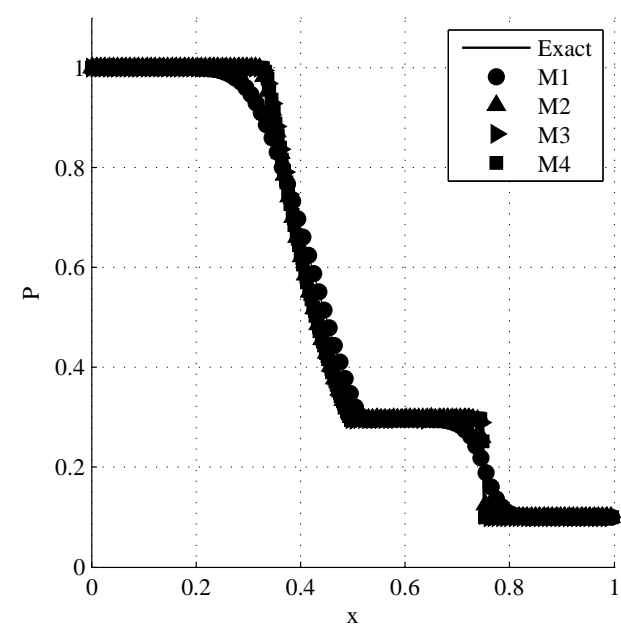

(a)

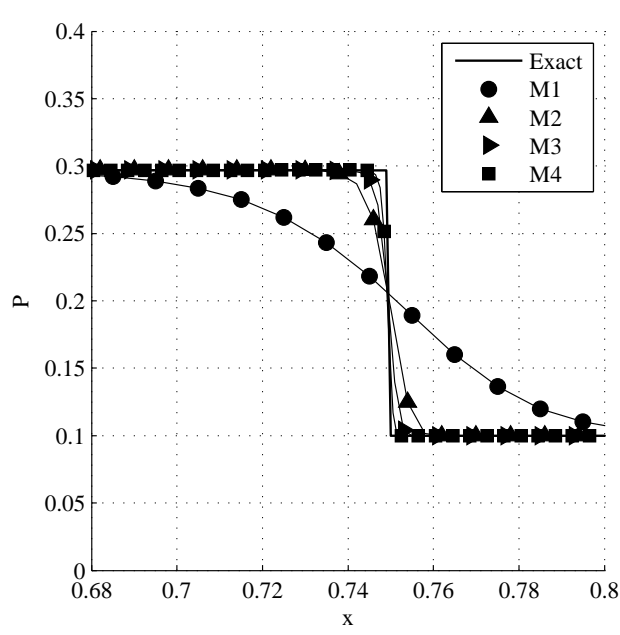

(b)

FIGURE 4.3: Pressure distribution for non reactive shock tube simulation DBS-AUSM-GC-2U and the influence of mesh cell size. Complete domain (a) and zoom on shock wave front (b)

The comparison between the theoretical and the predicted shock wave velocities provide insight into the performance of the numerical model. However, further characterization can be achieved by quantifying the numerical diffusion throughout the domain. In order to separate the error from the over prediction of the shock wave velocity and the numerical diffusion, the predicted pressure distributions were translated in the x-axis. Such translation was performed in order to match the location of the shock wave front from the numerical predictions to that of the analytic solution. It is worth mentioning that the shock wave front from the numerical predictions was defined as the mid point between the two computational cells that presented the maximum pressure gradient in the $\mathrm{x}$-direction, as given by Equation 4.1.

$$
x_{S W, C F D}=\left(\frac{x_{i}-x_{i-1}}{2}\right) \text { for }\left.\frac{\partial P}{\partial x}\right|_{i}=\max \left(\frac{\partial P}{\partial x}\right)
$$

Translation of the pressure distribution allows for the calculation of the local error of the pressure prediction. The error is then calculated based on Equation 4.2.

$$
E_{P}(x, t)=\left|\frac{P_{C F D}(x, t)-P_{\text {exact }}(x, t)}{P_{\text {exact }}(x, t)}\right|
$$

It was obtained that the local error is larger in the vicinity of the shock wave front and in the region that has been affected by the expansion wave. Figure 4.4 shows the distribution of the local error for simulations using DBS, AUSM, SQC and 2U; with different mesh resolutions. Error from M1 solution is not included in the figure, since the maximum error is of the order of 0.9 ; if included the error for M2, M4 and M4 predictions cannot be appropriately appreciated. 


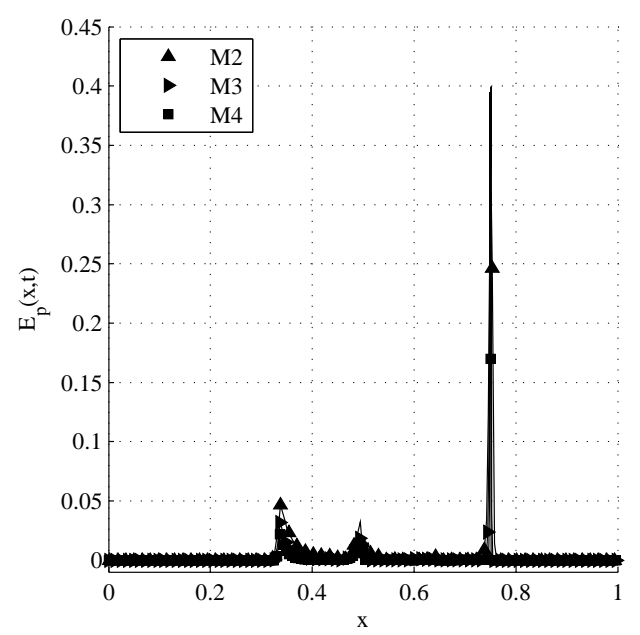

(a)

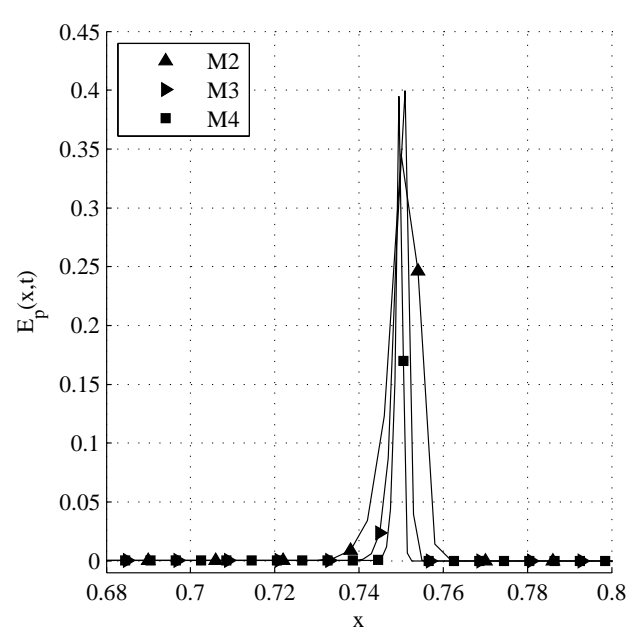

(b)

FIGURE 4.4: Local error distribution for non reactive shock tube predictions using DBS-AUSM2UP and the influence of mesh cell size. Complete domain (a) and zoom on shock wave front

The calculation of the local errors permits assessment of the performance of the numerical model under a specific flow condition in the domain. However, it is necessary to obtain an integral quantification of the error in order to judge the overall convergence of the numerical model. This is done by calculating the $\ell^{2}$-norm of the error using Equation 4.3 as given in [Celik, 2007].

$$
\ell^{2} \text { norm }=\sqrt{\frac{\sum E_{P}(x, t)^{2}}{N_{x}}}
$$

The calculated $\ell^{2}$-norm for each mesh resolution is then related to the normalized cell size; given by the ratio of the current cell size divided by the minimum cell size in the $\mathrm{x}$ direction $\left(\frac{\delta x}{\delta x_{\min }}\right)$. Figure 4.5 exemplifies the spatial error $\ell^{2}$-norm for the pressure obtained by the simulations using DBS, AUSM, SQC and $2 \mathrm{U}$, with different mesh resolutions.

Lines representing first and second order convergence are added in Figure 4.5. It is observed that the calculated error data falls between these two lines, but closer to the first order slope. A specific quantification of the convergence order is performed by fitting a power-law function to the error data. This was performed using the least square method (Figure 4.5). The fitted function illustrates that the degree of convergence of the method results to be 1.16. Therefore it is concluded that the method is first order. This can be explained by the fact that although, $2^{\text {nd }}$-Order-Upwind discretization scheme was used, the convergence criteria remains close to 1 due to the use of first order temporal discretization. 


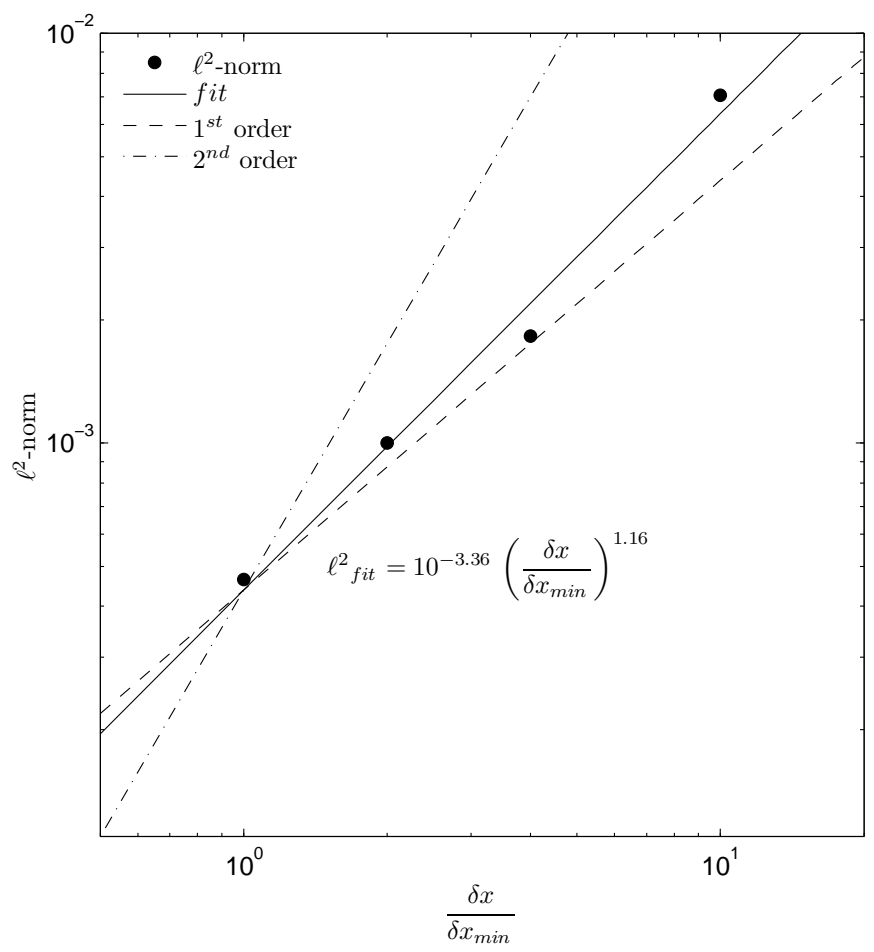

FIGURE 4.5: Spatial error $\ell^{2}$-norm for non reactive shock tube using DBS, AUSM, SQC and 2U

\subsubsection{Results using the Pressure Based Solver (PBS)}

In addition to the analysis performed for results obtained using the DBS, a similar study was carried out for the PBS; where two pressure-velocity coupling algorithms, two gradient calculation methods and four discretization schemes were considered. The influence of the different solver configurations was evaluated by performing a total of 16 numerical simulations. The results show that the PBS, under all the studied configurations, appropriately predicts the general behavior of the flow for a non reactive shock tube problem. When PBS is used, the pressure distribution along the $\mathrm{x}$-axis does not show any significant difference when compared to the solutions using the DBS. Additionally, no difference in the prediction of the shock wave velocity due to the changes in discretization schemes is observed. For all the studied configurations the shock wave velocity was predicted with a relative error of $2.4 \%$. The complete list of the configurations that were studied and their corresponding results for shock wave velocity are shown in Table C.3. The influence of the mesh resolution on the prediction of the shock wave velocity using the PBS is given in Table C.4. Once more, the results do not present any significant difference when compared to those obtained using the DBS. The error analysis for the results obtained using the PBSwas also performed. The $\ell^{2}$-norm of the error as a function of the mesh resolution for the pressure predictions using the pressure based solver (PBS) is shown in Figure ??. It is observed once again, that the degree of convergence of the implemented method is essentially the same as that of the DBS solver (i.e. First Order) 


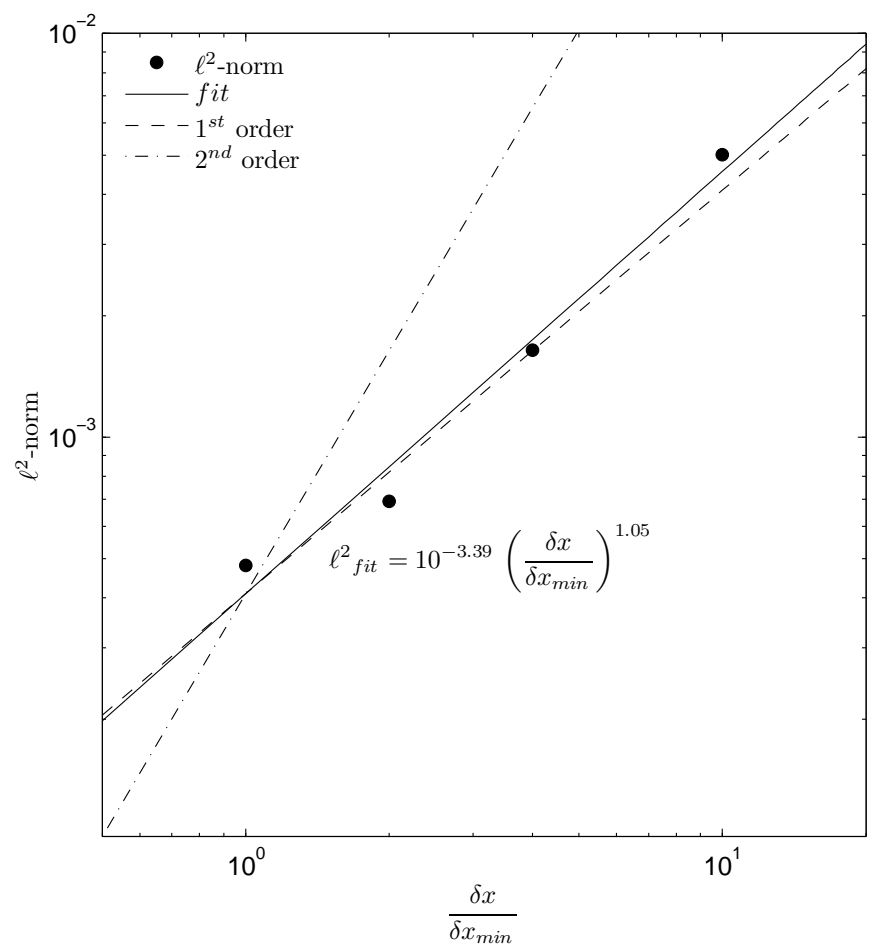

FigURE 4.6: Spatial error $\ell^{2}$-norm for non reactive shock tube using PBS, PISO, SQC and 2U

In addition to the First Order temporal discretization scheme, simulations using Second Order Discretization Scheme for the non reactive shock tube problem were performed. It was obtained that "wiggles" appear in the predicted pressure behind the shock wave front. The fluctuations predicted for the pressure behind the shock wave are illustrated in 4.7.Through the evaluation of the $\ell^{2}-$ norm for the pressure in the error prediction, and as illustrateed in Figure 4.6, it was obtained that despite implementing second order time discretization, the actual order of convergence is equal to 1.0 .

The following conclusions can be drawn from analysis of the numerical prediction for non reactive shock tube problem: First, both the DBS and PBS can capture the flow discontinuities present in compressible flows. Second, the shock wave velocity is consistently over predicted when either PBS or DBS solvers are used. However, the error based on the pressure prediction decreases as the cell size decreases. The third, is that although second order schemes (2UP) are used the convergence of the method based on the $\ell^{2}$-norm of the error in the pressure prediction reports an first order convergence. Such convergence order was also observed for second order temporal discretionary scheme. Based on the results obtained in Section 4.1, the numerical strategy for the simulations presented in the following portion of the current study was selected. Simulations using DBS were performed with DBS-AUSM-SQC-2UP, and those with the PBS were performed with PBS-PISO-SQC-2UP. 


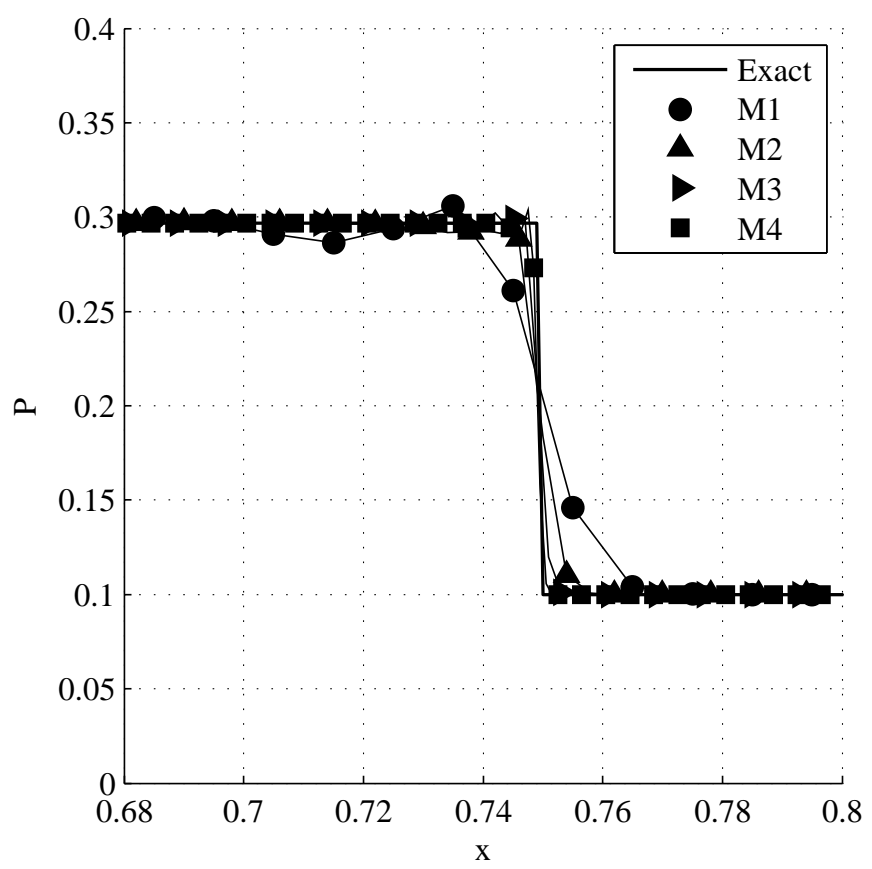

(a)

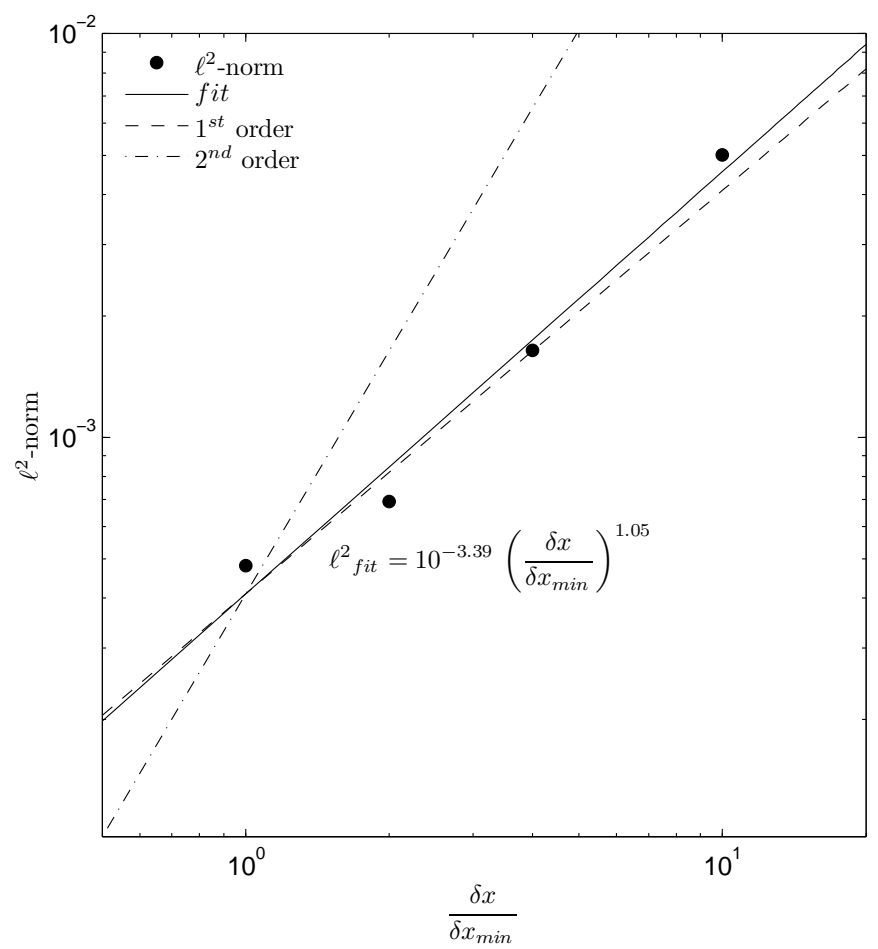

(b)

FIGURE 4.7: 2D CFD shocktube simulation results PBS-PISO-SQC-2U and 2nd Order in time. Pressure distribution (a) and 12 norm of the pressure error (b) 


\subsection{Detonation Tube Simulations}

As described in Section 2.1, the propagation of a detonation wave in a premixed gas is an inherently unstable and a 3D phenomena. The presence of small fluctuations and disturbances on the distribution of the flow velocity, temperature and chemical composition of the gas ahead of the detonation front lead to the appearance of cellular instabilities. The prediction of the detailed detonation wave structure is not the goal of the current research work, instead it focused on predicting the overall parameters of the detonation wave and on assessing the influence of various modeling assumptions on such predictions. Therefore the problem selected for validation corresponds to the prediction of a planar 2D detonation wave, where the gas ahead of the detonation wave is prescribed as uniform in its temperature, velocity and chemical composition.

After studying the performance of different solver configurations on the prediction for the non reactive shock tube problem, the second validation test is presented. This study consists of the prediction of the propagation of a planar detonation wave in a 2D channel. In this section the influence of different model parameters on the prediction of the CJ detonation wave velocity $\left(U_{C J}\right)$, pressure $\left(P_{C J}\right)$ and temperature $\left(T_{C J}\right)$ are studied. Therefore, simulations using two different solvers (PBS and DBS), two types of chemical reaction models and four different mesh resolutions are evaluated. The CFD results from the various numerical configurations are then compared with the CJ and ZND predictions for the mixtures and conditions of interest. As a result, a modeling strategy capable of predicting the propagation of a detonation wave while maintaining low computational cost is identified.

\subsubsection{Influence of chemical model on $\mathrm{CJ}$ and ZND predictions}

Prior to the description of the detonation tube problem and the presentation of the CFD results, the CJ and ZND solutions for the mixture of interest are presented. As mentioned in Section 2.1, the calculation of the $\mathrm{CJ}$ detonation state does not require the information of the chemical kinetics. However the CJ state is - indeed - a function of the species considered and their thermodynamic properties. Thus, the CJ state prediction is a function of the selected chemical reaction model in the sense that it determines the included species and the way their thermodynamic properties are calculated. For example, the single step reaction mechanism described in Section 3.1, groups the detonation products in one surrogate molecule (R), and its thermodynamic properties are defined as mentioned in the forementioned section. On the other hand, the multi-step reaction mechanism has a predefined number of species that are considered (14 for the $\mathrm{H}_{2}$-air mechanism). The thermodynamic data for the species in the multi step reaction mechanisms are calculated using JANAF polynomials [Chase and Force, 1998]. 
The CJ state for the chemical modeling strategies considered were predicted and the results are given in Table 4.2. It is observed that the predictions of $U_{C J}$ and $P_{C J}$ for $\mathrm{H}_{2}$-Air mixture do not present significant differences between the two chemical reaction models, $0.2 \%$ and $4.8 \%$ respectively. However, $T_{C J}$ is under predicted by $6 \%$, while using the one step reaction model, compared to the CJ temperature calculated using [Gordon and McBride, 1972] and considering a total of 30 species.

TABle 4.2: Chapman-Jouguet Prediction from the different chemical modeling strategies for $\mathrm{H}_{2}$-air and syngas-air mixtures

\begin{tabular}{ccccc}
\hline \hline Mixture & Reaction Model & $\begin{array}{c}U_{C J} \\
\mathrm{~m} / \mathrm{s}\end{array}$ & $\begin{array}{c}P_{C J} \\
\mathrm{~atm}\end{array}$ & $\begin{array}{c}T_{C J} \\
\mathrm{~K}\end{array}$ \\
\hline$H_{2}$-Air & 1-step & 1973 & 15.82 & 2769 \\
$H_{2}$-Air & Multi-step & 1969 & 15.1 & 2923
\end{tabular}

To further analyze the differences, ZND calculations were performed for stoichiometric $\mathrm{H}_{2}$-air mixtures. The ZND predictions provide the means to compare the predicted length and time scales for the planar stable detonation wave structure. Table 4.3 shows the predicted length scales obtained using the single and multiple step reaction mechanism. The calculations for the multi-step reaction mechanisms were performed using [Goodwin, 2005], while the single step ZND structure was calculated using the methodology described in [Lee, 2008]. In addition to the values of the length scales, Figure 4.8 presents a comparison of the distribution of temperature, pressure and thermicity for the ZND predictions.

TABLE 4.3: ZND Prediction for $\mathrm{H}_{2}$-Air using the different chemical modeling strategies

\begin{tabular}{ccc}
\hline \hline Reaction Model & $\Delta_{I}$ & $\Delta_{R}$ \\
& $\mathrm{~m}$ & $\mathrm{~m}$ \\
\hline 1-step & $6.23 \times 10^{-5}$ & $2.28 \times 10^{-4}$ \\
Multi-step & $1.95 \times 10^{-4}$ & $4.5 \times 10^{-4}$ \\
\hline
\end{tabular}

The information in Table 4.3 and Figure 4.8 evidence the differences in the detonation wave structure for the predictions obtained using the 1 step and the multi-step reaction model. One of the differences is the fact that the ZND structure calculated using the 1 step reaction model does not present an induction region. This means that, as illustrated in Figure 4.8(a), the near isothermal zone directly behind the leading shock wave $(x=0 \mathrm{~mm})$ is only predicted when the multi-step reaction model is implemented. In the case of the predicted ZND structure using the 1 step reaction mechanism, the temperature increases immediately behind the leading shock. The steep increase in temperature can be directly correlated to the distribution of heat release depicted by the distribution of thermicity (Figure 4.8(d)). The heat release for the prediction with a 1 step reaction mechanism begins immediately behind the shock wave. This difference can be explained by the reaction rate for the 1 step reaction (Equation 3.7). It is observed that the reaction rate for the 1 step reaction model is only a function of the molar concentration of 


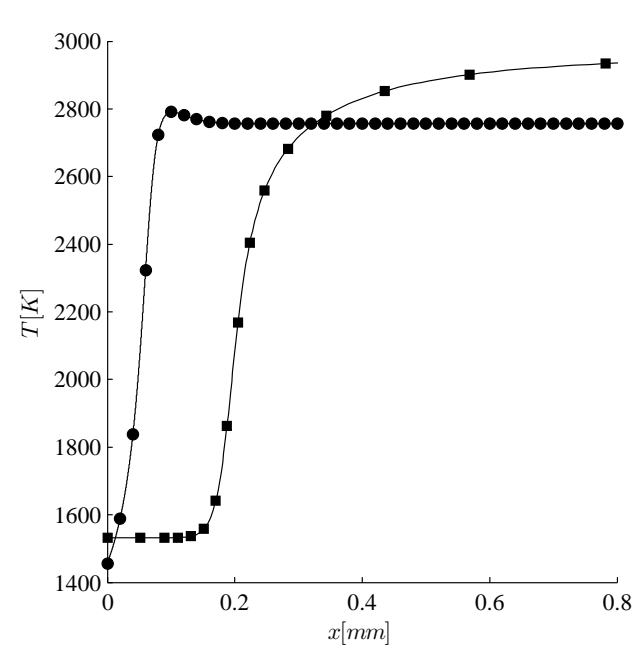

(a)

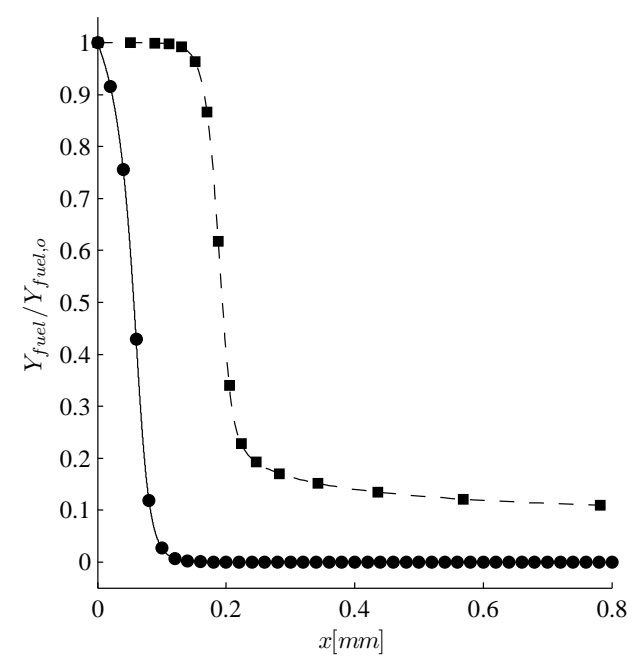

(c)

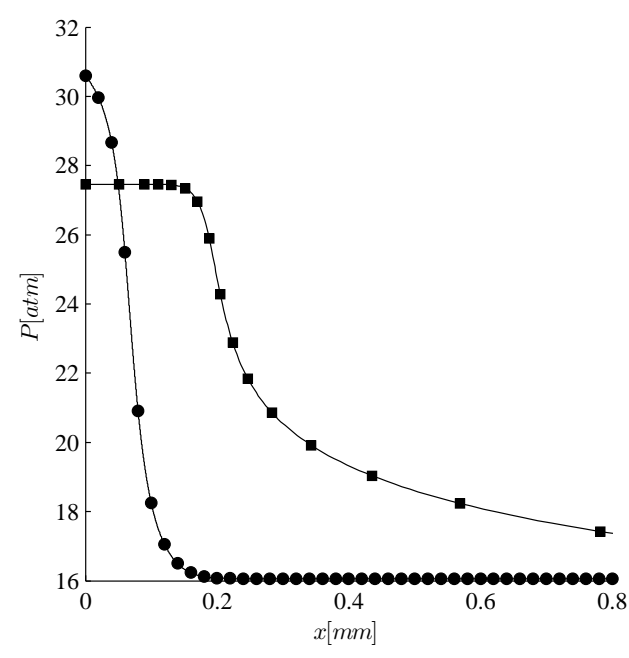

(b)

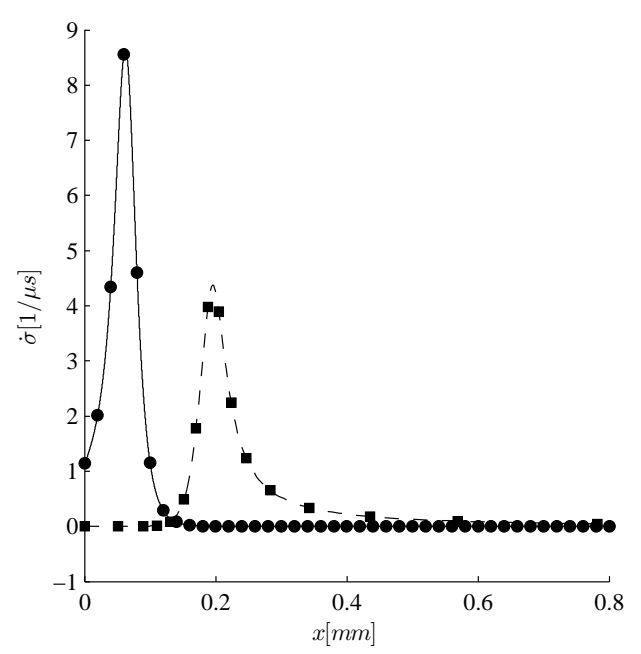

(d)

FIGURE 4.8: ZND prediction for stoichiometric $\mathrm{H}_{2}$-air with single step (๑) and multistep reaction models( $\mathbf{\square})$. Temperature (a), pressure (b), normalized fuel mass fraction (c) and thermicity (d) distribution.

the reactants and the temperature of the mixture. Hence, the consumption of reactants is immediately onset by the increased temperature state of the gases behind the shock wave. Instead, when the multi-step reaction model is implemented; the high temperature of the gases on the post shock state induces the production of radical species and not the direct consumption of the fuel molecule. For the multi-step reaction model, the fuel is consumed once the radical concentration is favorable for such process. The previously mentioned behavior is also supported by the the normalized fuel fraction distribution illustrated in Figure 4.8(c), where the depletion of fuel for the 1 step reaction model occurs in a much restricted span. In addition to the onset of heat release and fuel consumption, it is observed that the location of the maximum thermicity point for the 1 step reaction mechanism occurs much closer to the shock front than the multi-step reaction mechanism. The before mentioned situation leads to the differences in length scales 
shown in Table 4.3.

Despite the differences in the ZND predictions for the different chemical models, both strategies show adequate prediction of the governing parameters of a detonation wave. A better prediction of the ZND structure is obtained while a multi-step model is implemented. However, a larger number of species make that reaction model more computationally expensive. The drawbacks of the 1 step reaction model do not signify its application is inappropriate. It is just to note that by knowing its limitations a better analysis can be performed for the CFD results obtained. Additionally, the low computational cost make this strategy very suitable for application in parametric studies and 3D simulations.

Once the differences have been established, it is possible to cover the CFD solution of a planar detonation wave in a $2 \mathrm{D}$ channel. The problem consists of a $2 \mathrm{D}$ rectangular domain with a lentgh of $400 \mathrm{~mm}$ and a height of $30 \mathrm{~mm}$ in which a detonation wave propagates on a premixed fuel and oxidizer environment. The domain at the initial condition is separated in two regions: Region I as the initiation zone and Region II as the premixed fuel and oxidizer portion of the domain. The initiation zone is initially patch with pressure, temperature and species values correspondent to the CJ state of the mixture of interest. Meanwhile, Region II is patched with stoichiometric mixture of fuel and oxidizer at standard conditions. A detonation wave forms and propagates from Region I into Region II. A schematic representation (Figure 4.9) illustrates the division of the domain and the flow structure after the detonation wave has propagated.

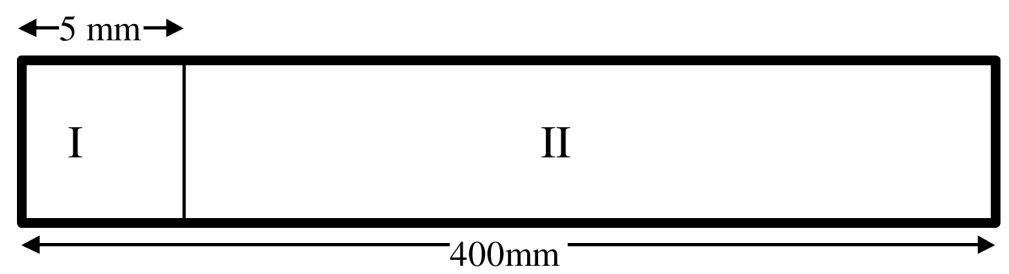

(a)

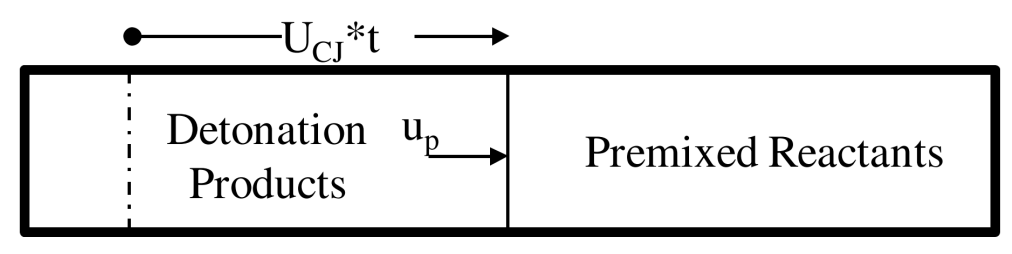

(b)

FIGURE 4.9: Schematic for the detonation tube problem, initial condition (a), flow structure at time t (b)

The boundaries of the computational domain illustrated on Figure 4.9 are treated as wall boundaries. Due to the fact that the simulations were performed under the assumption of neglectable viscous effects, the walls were specified with a slip condition. 
The domain was then discretized into a computational mesh with orthogonal quadrilateral elements. As part of the present study, the performance of four different computational cell sizes $(\delta x)$ were evaluated. The considered sizes are $0.5,0.25,0.1$ and $0.05 \mathrm{~mm}$. The time step $(\delta t)$ for the studied meshes was selected in order to maintain the CFL number lower than 2.

Similar to the work presented in Section 4.1, different numerical solution strategies were analyzed. The numerical simulations were performed using either the DBS or the PBS. For the simulations where the DBS solver was employed, the AUSM flux calculator, the SQC gradient scheme and the $2 \mathrm{U}$ discretization scheme were implemented. On the other hand, when the simulations were carried out using the PBS the PISO P-v coupling algorithm, the SQC gradient scheme and the 2UP scheme were applied. As regards to the chemical model, both the 1 step reaction and the multi-step reaction model were implemented into the DBS simulations. However, the multi-step reaction mechanism was not utilized while using the PBS solver, due to the fact that the time splitting algorithm for the treatment of the chemical source does not function properly while using the PBS. All the numerical configurations presented in this section, led to the solution of a self-sustained detonation wave.

A characteristic pressure and temperature distribution for the prediction of a detonation wave in a $2 \mathrm{D}$ channel is given in Figure 4.10. The data illustrated corresponds to a computational mesh with $\delta x=0.05 \mathrm{~mm}$. The pressure and temperature prediction follow the expected trend for a detonation wave, where a distinct reaction front is captured and the appropriate expansion of the detonation products are predicted. On the temperature distribution (Figure 4.10(b)), the high temperature region located on the left end of the detonation tube $(x<10 \mathrm{~mm})$, is the remainder of the high temperature patch from the initial condition.

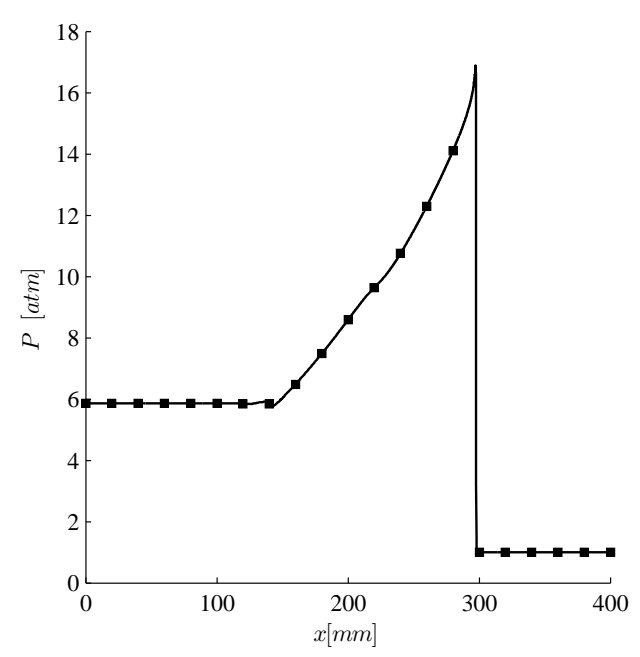

(a)

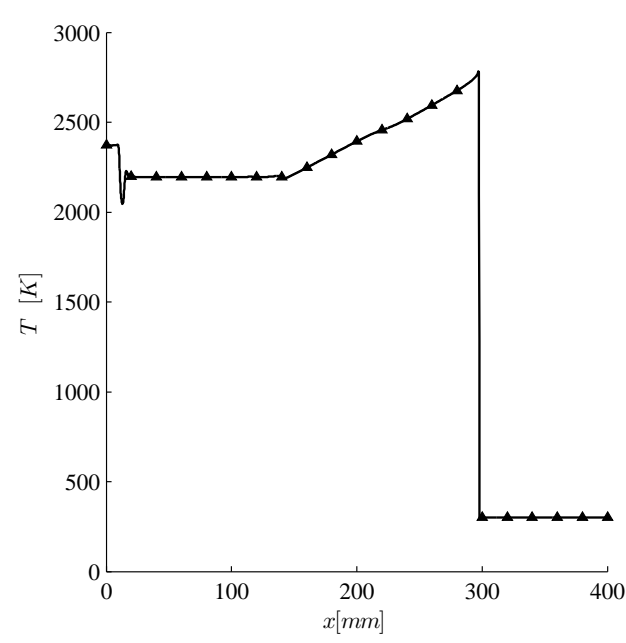

(b)

FIGURE 4.10: Planar detonation wave CFD prediction for 1 step reaction mechanism with PBSPISO-SQC and $\delta x=0.05 \mathrm{~mm}$. Pressure (a) and temperature (b) distribution 
A better judgment on the predicted detonation wave structure can be performed by analyzing the temperature pressure and heat release distribution in the region near the detonation wave front. Figure 4.11 shows that the 2D CFD simulations predicts a pressure wave coupled with a heat release front. Since the spatial resolution in the CFD simulations is not as refined as those used for the ZND predictions, it is not expected to capture the same degree of detail. However, the predicted detonation wave structure imitates that of the ZND model. In addition to the analysis of the spatial distribution for pressure, temperature and heat release; the detonation wave velocities $\left(U_{D W}\right)$ were calculated. For such calculations, pressure monitors were placed along the $\mathrm{x}$-direction of the computational domain at half of the $2 \mathrm{D}$ channel's height. Then, the pressure history from the monitor probes was stored, and the detonation wave velocity was calculated using the time lapsed between the peak of the two signals. For the simulation illustrated in Figure 4.10, an average detonation wave velocity of $1964 \mathrm{~m} / \mathrm{s}$ was obtained.

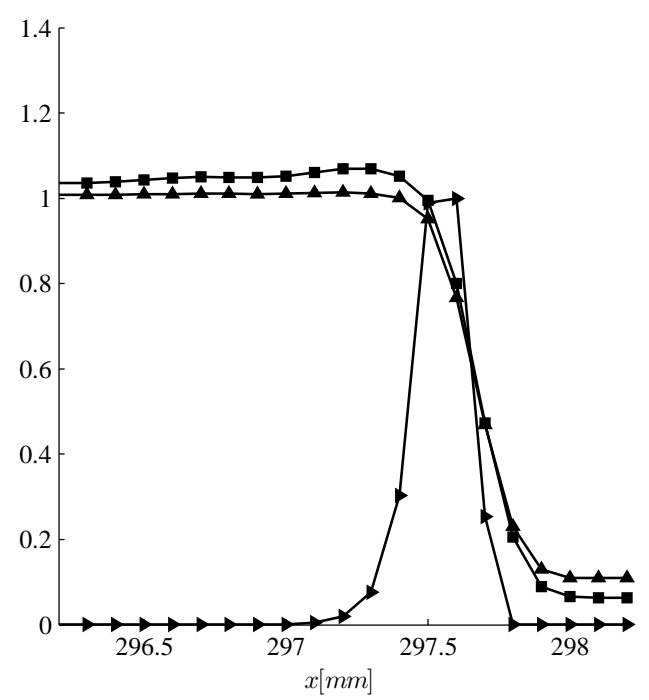

FIGURE 4.11: 2D detonation wave CFD prediction for 1 step reaction mechanism, region near the detonation front. Distribution of normalized pressure $\left(\frac{P}{P_{C J}}\right)[\boldsymbol{\square}]$, normalized temperature $\left(\frac{T}{T_{C J}}\right)[\mathbf{\Lambda}]$ and normalized thermicity $\left(\frac{\dot{\sigma}}{\dot{\sigma}_{\max }}\right)[\boldsymbol{}]$

The simulations were performed using different levels of mesh resolution. A summary of the results obtained using the PBS-PIOS-SQC-2UP configuration are presented in Table 4.4. The detonation wave velocity calculated based on the pressure history is included, together with the predicted $\mathrm{CJ}$ pressure and temperature. The values for $\mathrm{CJ}$ pressure and temperature from the CFD simulations were calculated based on the pressure and temperature distribution shown in Figure 4.10. The calculation process was based on determining the location at which the detonation products reach a Mach number, in the wave fixed reference frame, equal to 1 . As observed in Table 4.4, predictions obtained with mesh resolutions lower than $0.1 \mathrm{~mm}$ did not lead to the presence of a sonic line. Although sustained detonation was achieved using computational cell sizes larger than $0.05 \mathrm{~mm}$, the Mach number in the wave frame of reference reached maximum values of 0.98 but did not reach to values equal or greater than 1 . On 
the other hand, the results obtained with lower mesh resolution led to the prediction of the detonation wave velocity with an error of less than $2 \%$ when compared to $U_{C J}$. As such, they should not be disregarded as non valid. Instead, low resolution calculations can be employed, but the identification of their limitation and quantification of the error permit them to be used with caution. Additional to the uniform mesh strategy, the results for AMR are also presented in Table 4.4.

TABLE 4.4: CFD simulation of detonation wave prediction in a 2D channel, results using PBSPISO-SQC-2UP and multiple mesh sizes.

\begin{tabular}{ccccccccc}
\hline \hline Case Name & $\delta x$ & $\delta t$ & $U_{D W}$ & $P_{C J}$ & $T_{C J}$ & $E_{U}$ & $E_{P}$ & $E_{T}$ \\
& $\mathrm{~mm}$ & $\mu s$ & $\mathrm{~m} / \mathrm{s}$ & $\mathrm{atm}$ & $\mathrm{K}$ & $\%$ & $\%$ & $\%$ \\
\hline M1-PBS-PISO-SQC-2UP & 0.5 & 0.125 & 2000 & $\mathrm{NA}$ & $\mathrm{NA}$ & 1.4 & $\mathrm{NA}$ & $\mathrm{NA}$ \\
M2-PBS-PISO-SQC-2UP & 0.25 & 0.125 & 2000 & $\mathrm{NA}$ & $\mathrm{NA}$ & 1.4 & $\mathrm{NA}$ & $\mathrm{NA}$ \\
M3-PBS-PISO-SQC-2UP & 0.1 & 0.0625 & 1961 & 15.6 & 2737.2 & 0.6 & 0.63 & 0.24 \\
M4-PBS-PISO-SQC-2UP & 0.05 & 0.0625 & 1964 & 15.6 & 2737.2 & 0.6 & 0.63 & 0.24 \\
AMR-PBS-PISO-SQC-2UP & AMR & 0.015 & 1972 & 15.8 & 2743.8 & 0.1 & $<0.01$ & 0.07 \\
\hline
\end{tabular}

Similar to the study performed using PBS, simulations with the DBS implementation were also carried out. The numerical configuration consisted of DBS-AUSM-SQC-2UP. In contrast to the solution obtained using the PBS, both the single step and multi-step chemical models were implemented. For the DBS, however, only three mesh resolutions were tested $(\delta x=$ $0.5,0.25$ and $0.1 \mathrm{~mm}$ ). The predicted detonation wave velocities as a function of mesh resolution and reaction mechanism are provided in Table 4.5. It is observed that numerical simulations using the DBS and the 1 step reaction mechanism lead to higher error in the prediction of the detonation wave velocity, when compared to those obtained using the PBS. It is also experienced that the prediction of the detonation wave velocity using the multi-step reaction mechanism and the DBS show a better performance for computational cells of $\delta x=0.25 \mathrm{~mm}$. The pressure and temperature distribution are given in Figure 4.12.

TABLE 4.5: CFD simulation of detonation wave prediction in a 2D channel, results for DBSAUSM-SQC-2UP, multiple mesh sizes and two chemical models.

\begin{tabular}{ccccc}
\hline \hline Case Name & Chem. Model & $\begin{array}{c}\delta x \\
\mathrm{M}\end{array}$ & $\begin{array}{c}U_{D W} \\
\mathrm{~m} / \mathrm{s}\end{array}$ & $\begin{array}{c}E_{U} \\
\%\end{array}$ \\
\hline M1-DBS-AUSM-SQC-2UP & 1 Step & 0.50 & 2016 & 2.2 \\
M2-DBS-AUSM-SQC-2UP & 1 Step & 0.25 & 2041 & 3.5 \\
M3-DBS-AUSM-SQC-2UP & 1 Step & 0.10 & 2030 & 2.9 \\
M1-DBS-AUSM-SQC-2UP-MS & Multi Step & 0.50 & 1925 & 2.3 \\
M2-DBS-AUSM-SQC-2UP-MS & Multi Step & 0.25 & 1972 & 0.2 \\
M3-DBS-AUSM-SQC-2UP-MS & Multi Step & 0.10 & 2011 & 2.1 \\
\hline
\end{tabular}

Simulation with multi-step reaction model for the the computational mesh of $\delta x=$ $0.1 \mathrm{~mm}$ was initialized from the solution on a coarser mesh. In the process of interpolation from one computational domain to the other, instabilities on the simulations were observed. It is 
believed that the present of these transients led to the over prediction of the detonation wave velocity for this specific case. Remainders were also present in temperature distribution, as shown in Figure 4.12(b).

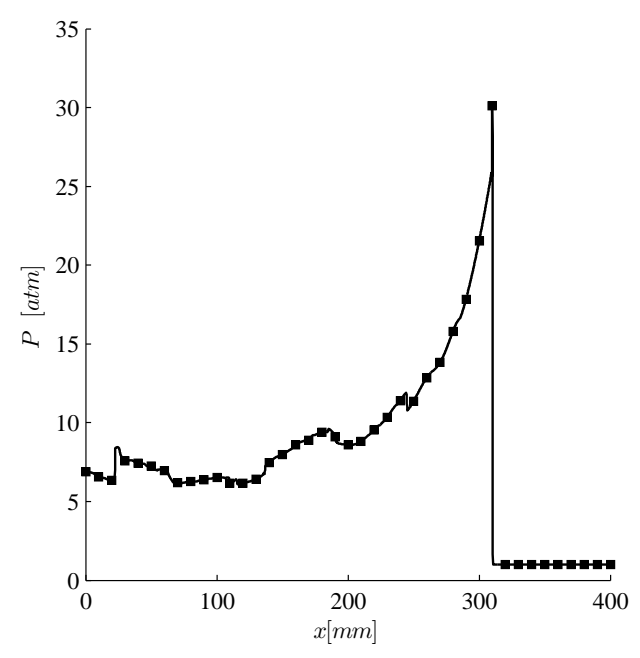

(a)

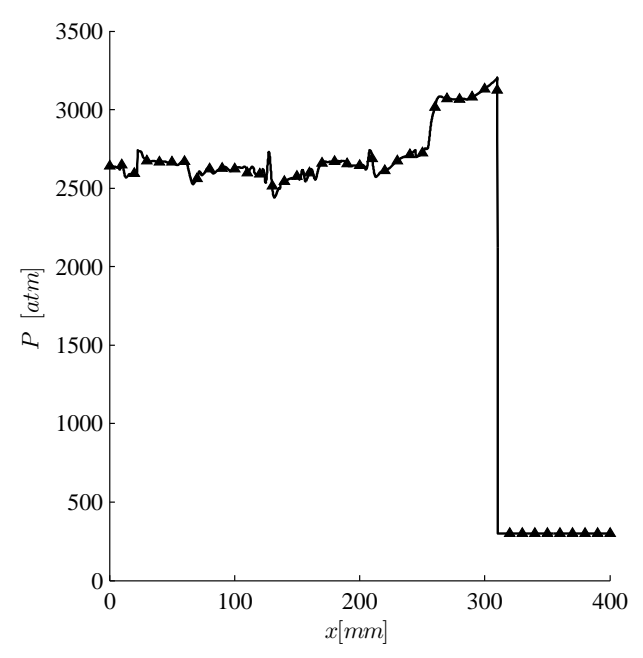

(b)

FIGURE 4.12: 2D detonation wave CFD prediction for multi step reaction mechanism, with DBS-AUSM-SQC-MS and $\delta x=0.1 \mathrm{~mm}$. Pressure (a) and temperature (b) distribution

In addition to the average detonation wave velocity, it is important to analyze the details of the predicted detonation wave structure. Figure 4.13(a) illustrates the normalized distribution of pressure, temperature and heat release in the proximity of the detonation wave front. Further more, a coupled structure of a pressure wave, heat release zone and the increase in temperature is predicted.

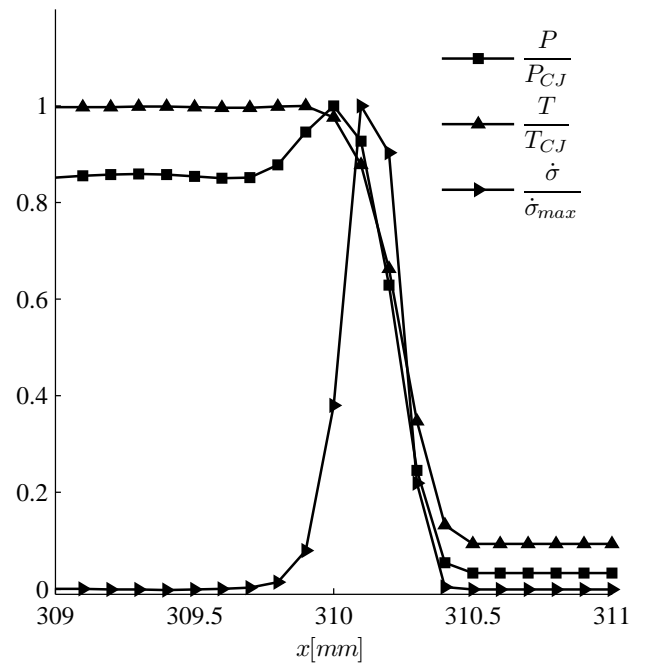

(a)

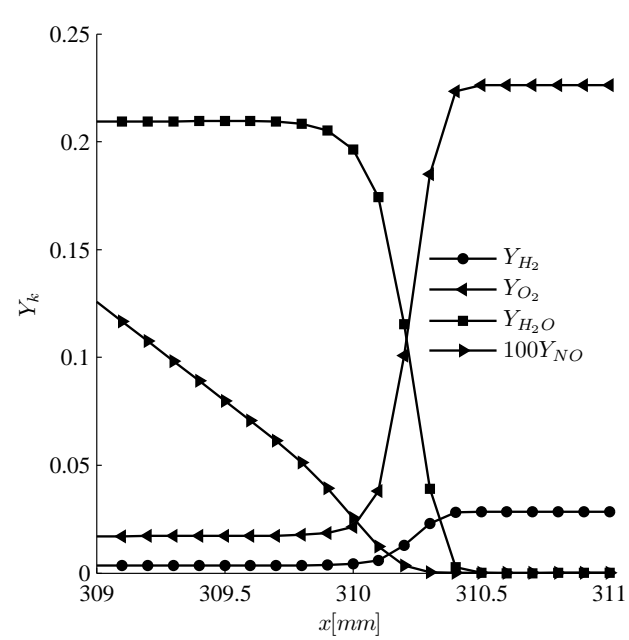

(b)

FIGURE 4.13: 2D detonation wave CFD prediction for multi step reaction mechanism, region near the detonation front. Normalized temperature, pressure and heat release distiibution (a), and distribution of species mass fraction (b) 
The mass fractions distribution for $\mathrm{H}_{2}, \mathrm{O}_{2}, \mathrm{H}_{2} \mathrm{O}$ and $\mathrm{NO}$ is given in Figure 4.13(b). It illustrates how the fuel $\left(\mathrm{H}_{2}\right)$ and oxidizer $\mathrm{O}_{2}$ react to form $\mathrm{H}_{2} \mathrm{O}$ in the region of the detonation wave front. Additionally it is observed that the NO mass fraction increases behind the detonation front. Although not shown in the the figure, the $Y_{N O}$ reaches a maximum value of $1.61 \times 10^{-2}$ further downstream of the reaction front, but decreases as the detonation products expand and temperature decreases.

The goal of this section of the current study is not to capture the characteristic 2D nor $3 \mathrm{D}$ detailed structure of the detonation wave. Instead, the presented simulations were performed to characterize the prediction of a planar detonation wave under different numerical models. In order to achieve such purpose, the field ahead of the wave was imposed as uniform without fluctuations in pressure, temperature or chemical composition of the gases. The lack of disturbances on the flow ahead of the detonation wave lead to the forementioned planar detonation wave (see Figure 4.10 and Figure 4.12). The stability of the numerical prediction was guaranteed by maintaining a CFL number lower than 2.5; therefore, pressure fluctuation behind the detonation wave were avoided. Nevertheless, if the time step was not reduced according to the level of mesh resolution, instabilities in the flow behind the detonation wave would appear. The strength of such numerical instabilities depended on the value of the CFL number. As an illustrative example, the prediction of the detonation wave for $\delta x=0.1 \mathrm{~mm}$ and $\delta t=1 \mu \mathrm{s}, \mathrm{CFL}=20.0$ is presented in 4.14. It is observed that $2 \mathrm{D}$ fluctuations are evident on the predicted pressure, temperature and density field behind the detonation wave. What is more critical is the fact that such instabilities resemble the behavior of the unstable physical nature of the propagation of a detonation wave, and they lead to detonation cell like structure behind the detonation front. These structures are not generated by the disturbances on the flow field ahead of the detonation wave, instead they are a product of numerical instabilities generated behind the detonation front. It is, therefore, important to guarantee that the instabilities predicted on a detonation wave front are not caused due to the inappropriate handling of the temporal and spatial discretionary of the problem.

It is then concluded that an adequate prediction of the detonation wave velocity is achieved. The results obtained using DBS and PBS show similar behavior, where the detonation wave velocity is predicted with relative errors lower than $4 \%$. The assessment of the error in the prediction of the propagation of a planar detonation wave permits the performance of a better analysis of the predictions obtained for RDC. Additionally, the detonation wave structure for both computational solvers imitates ZND predictions. However, the computational mesh resolution for the CFD calculations is not high enough to capture the detailed intricacy of ZND structures. In comparison, the predictions obtained using a 1 step reaction mechanism with the PBS show lower relative error of the detonation wave velocity as the mesh resolution is increased than those using DBS. Conversely, the use of the DBS to predict detonation wave phenomena with multi-step reaction mechanism was also proven satisfactory. The distribution 


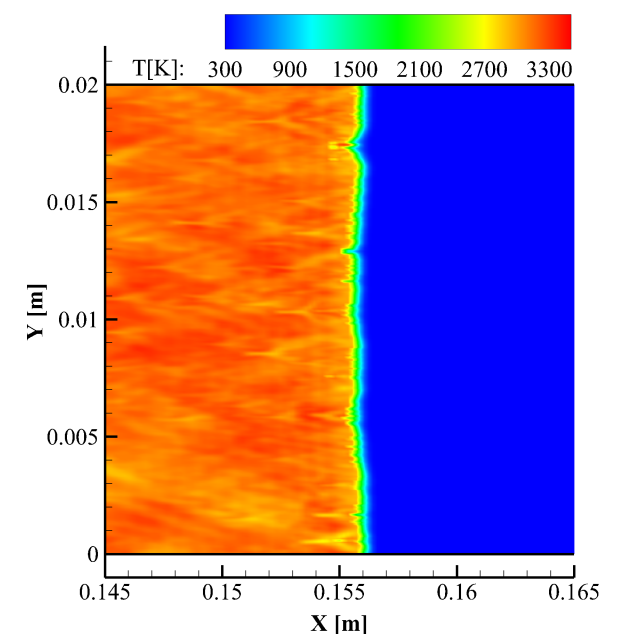

(a)

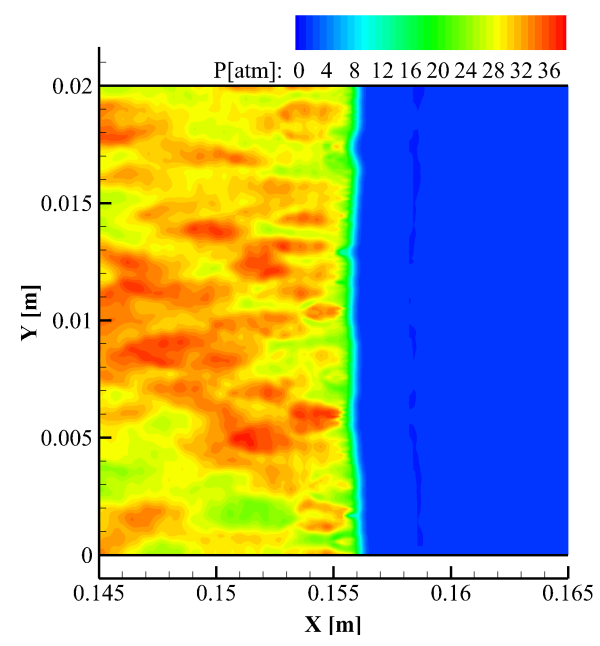

(b)

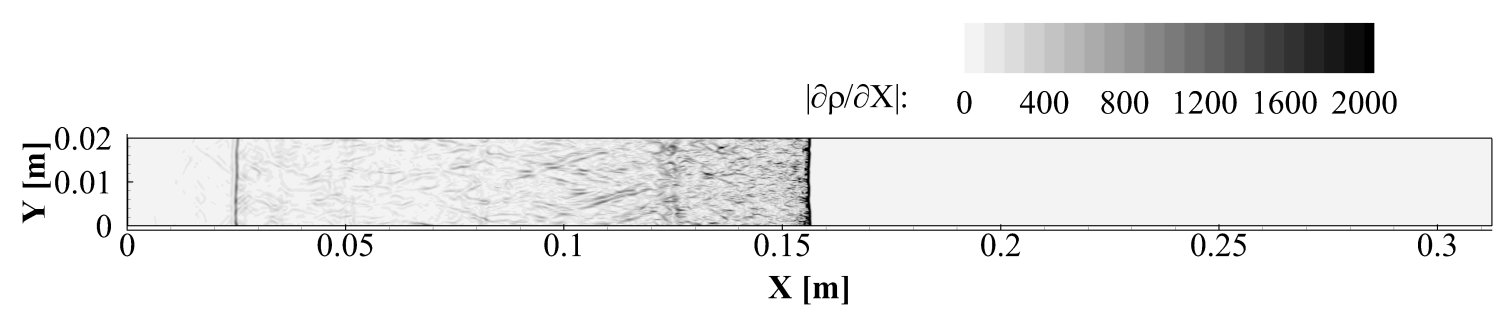

(c)

FIGURE 4.14: 2D CFD shocktube simulation results PBS-PISO-SQC-2U and 2nd Order in time. Pressure distribution (a) and 12 norm of the pressure error

of major and pollutant species shown in Figure 4.13 illustrate that the consumption of fuel and oxidizer is appropriately coupled with the high pressure and temperature front.

\subsection{Simulation of Rotating Detonation Combustion for Model Val- idation}

Once the performance of the computational methods considered for the present research initiative have been tested for relatively simple computational cases, the performance of these methods need to be tested for the simulation of RDC. In contrast to the study cases of non reactive shock tube and planar detonation wave, there is no exact solution for the RDC problem. Therefore, the validation of the computational model will be based on the comparison of qualitative and quantitative data from experiments reported by the scientific community.

The experimental data used for validation in this study was taken from the work by [Liu et al., 2012]. This selection was based on the detailed description of the experimental set up and combustor geometry, adequate characterization of the operating conditions, and suitable presentation of the experimental results. The combustor consists of an annular chamber formed 
by an outer cylinder with a diameter $\left(D_{\text {out }}\right)$ equal to $100 \mathrm{~mm}$ and a concentric inner insert of diameter $\left(D_{i n}\right) 90 \mathrm{~mm}$, and length $\left(L_{c}\right) 75 \mathrm{~mm}$. Reactants are injected into the combustion chamber through two different streams. A set of 90 equally spaced orifices with a diameter of $0.8 \mathrm{~mm}$ in the inner insert are used for the injection of hydrogen. Air stream is injected through an annular slot, with a width $\left(\Delta_{\text {air }}\right)$ of $0.4 \mathrm{~mm}$. A detailed illustration of the combustion chamber geometry is presented in Figure 4.15. Further details can be found in [Liu et al., 2012].
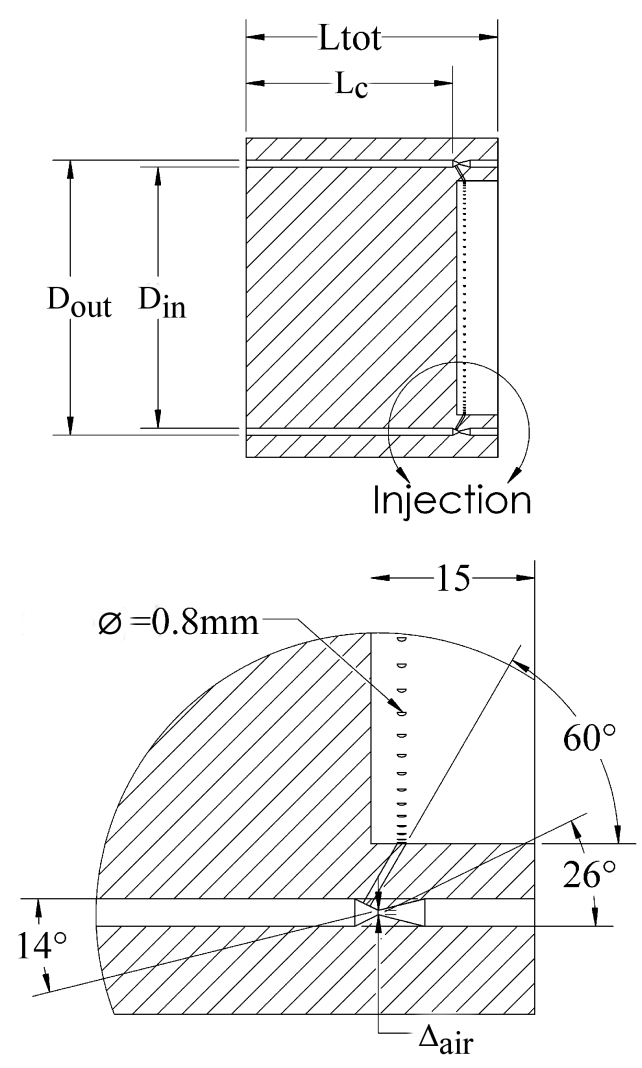

FIGURE 4.15: Schematic representation of combustor geometry for model validation

The average mass flow rate for hydrogen and air under stable operation is reported to be 7.7 and $265 \mathrm{~g} / \mathrm{s}$ respectively [Liu et al., 2012].The experimental flow rates provided in [Liu et al., 2012] were then used to calculated the required stagnation pressure of the injection manifold required to obtain an injection mass flow rate of $272.7 \mathrm{~g} / \mathrm{s}$, under the assumption of perfectly premixed stoichiometric $\mathrm{H}_{2}$-Air. The required total injection pressure is equal to $11.23 \mathrm{~atm}$. It was also assumed that the temporal variation of the stagnation state at the injection manifold is negligible. The ratio between fuel and oxidizer correspond to that for stoichiometric conditions.

Experimental data reported in [Liu et al., 2012] consists on two types of pressure measurements: low sampling rate pressure measurements for average chamber pressure, reactants manifold, and reservoir pressure. Additionally, two dynamic piezo electric transducers (PCB 113 B2411) were used to measure the instantaneous pressure inside the combustion chamber. 
Although the experimental set up used in [Liu et al., 2012] did not include visual access to the interior of the combustion chamber, results similar to those presented in [Bykovskii et al., 2009] provide the opportunity to qualitatively compare the detonation wave flow structure from photo recordings to the computational results of this study.

\subsubsection{Two Dimensional Simulations of RDC for Model Validation}

The first set of results presented for the model validation on the prediction of RDC corresponds to two dimensional CFD simulations. For this set of numerical studies, the computational domain was simplified as described in Section 3.3, and as illustrated by Figure 3.2. The resulting 2D domain was then partitioned into a computational grid as presented in Figure 3.3. As part of the solution strategy, the $2 \mathrm{D}$ simulations were initially solved on a computational mesh with a cell size $(\delta x)$ of $0.5 \mathrm{~mm}$ and the initial condition was prescribed according to the process described in Section 3.5. Once the transients from the initial conditions were eliminated, and continuous detonation was guaranteed; the solution was interpolated into a finer mesh $(\delta x=0.1 \mathrm{~mm})$ and the simulation was completed using the finer mesh. In order to capture the periodic steady state solution, the simulation was calculated for at least three additional detonation wave cycles before using the data for post processing.

The boundary conditions implemented in the 2D computations correspond to those described in Section 3.4. The inlet boundary condition is prescribed according to the model developed by [Zhdan et al., 1990] and the outlet boundary was set as a pressure outlet with a far field pressure $\left(P_{\infty}\right)$ of $0.11 \mathrm{~atm}$. Due to the low value for the far field pressure, the detonation products exit the chamber at supersonic conditions and the implementation of NRBC is not required. Due to the $2 \mathrm{D}$ nature of this set of simulations, the vertical boundaries are set as periodic boundaries.

Two types of monitoring strategies were used for the simulations. One consisted of placing numerical probes distributed in the computational domain. The probes or monitor points were placed in two colinear groups separated by $\frac{\pi D_{\text {mean }}}{2}$. The static pressure was sampled from these numerical monitors, and the data was used to calculate the detonation wave velocity and the average static pressure distribution in the axial direction of the combustion chamber. The second monitoring strategy consisted of sampling integral values at the inlet and outlet of the computational domain. Quantities such as the averaged stagnation pressure $(\hat{P})$, and the inlet and outlet mass flow rates were sampled as a function of time. These integral quantities were then used to calculate performance parameters of RDC.

The results that are first covered in this subsection correspond to the 2D simulations obtained using the PBS with the 1 step reaction mechanism. The solver configuration was selected based on the solution of the planar detonation wave simulations addressed in Section 4.2, and it corresponds to the use of PBS-PISO-SQC-2UP. Initial validation of the results obtained using 
this solver configurations are performed by studying the predicted temperature field on the $2 \mathrm{D}$ domain. For such purpose, Figure 4.16 shows the temperature distribution of 2D simulation for stoichiometric $\mathrm{H}_{2}$-air under the operating conditions corresponding to the experimental work in [Liu et al., 2012]. The detonation front traveling from right to left can be identified from Figure 4.16. The detonation front height $\left(\Delta_{D W}\right)$ is then defined by the axial distance that the unreacted fuel and oxidizer mixture reach during once cycle of the detonation wave. The oblique shock wave and shear layer can be identified as the high temperature region extending from the upper edge of the detonation front into the outlet of the domain.

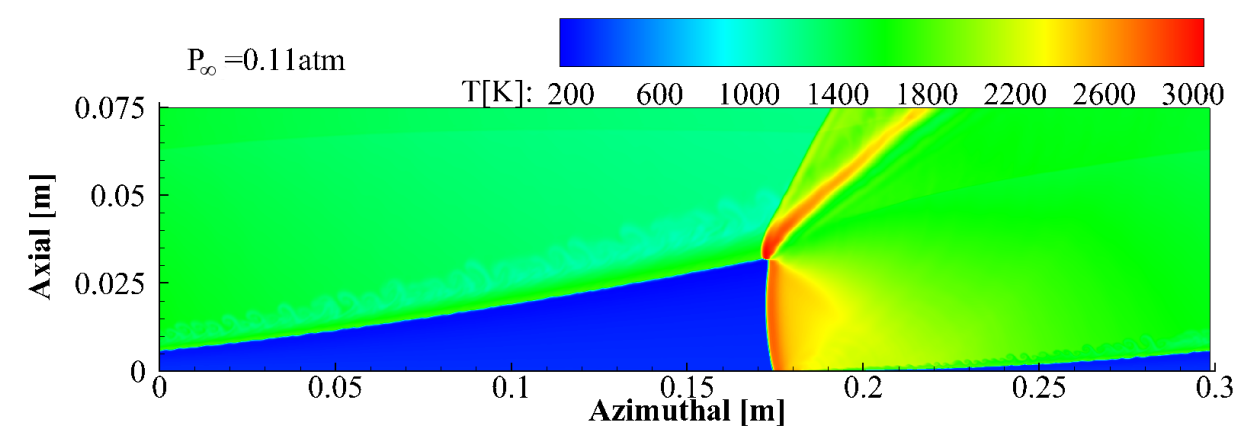

FIGURE 4.16: 2D temperature contour for PBS-PISO-AUSM-2UP RDC prediction for model validation

Based on the temperature contour shown in Figure 4.16, it is possible to determine that there is quantitative agreement between the predicted main flow features from CFD and the experimental observations. However, additional investigation of the predicted flow field yield a better insight of the flow pattern inside RDC. Hence, the results from the density field are post processed by calculating the magnitude of the density gradient vector. The resulting density gradient $\left(\left|\frac{\partial \rho}{\partial \vec{x}}\right|\right)$ field is then illustrated as a Schlieren-like contour plot in Figure 4.17. It is then observed that the detonation wave is depicted as a region where a sharp density gradient occurs, which is also accompanied by the combustion front formed between the unreacted mixture and the detonation products. In addition to the oblique shock wave that extends from the end of the detonation front towards the outlet, a second pressure wave forms from the edge of the detonation front extending in the azimuthal direction of the chamber. This pressure wave is observed as a thin density gradient region in Figure 4.17, extending from the edge of the detonation front to the right periodic boundary and appearing on the left hand side. It finally weakens in the interaction with the oblique shock. The detonation products remain supersonic despite the presence of the azimuthal pressure wave.

In addition to the presence of the discontinuities, it is also interesting to point out the eddy structures that form downstream of the combustion front. In order to further assess these structures, stream lines based on the fixed wave velocity field were created. The streamlines location was selected such that one would be located in the detonation product region and the other in the unreacted region. The location of the streamlines is illustrated in Figure 4.18(a). 


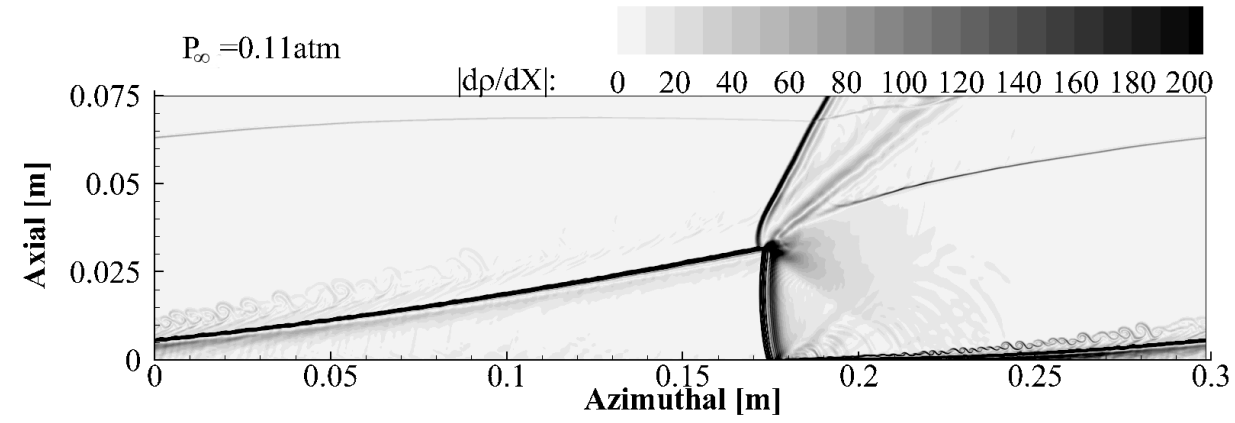

FIGURE 4.17: 2D $\left|\frac{\partial \rho}{\partial \vec{x}}\right|$ contour for PBS-PISO-SQC-2UP RDC prediction

Then, the density and velocity distribution along the streamlines were extracted and plotted in Figure 4.18(b)(c). It is observed that there is a large difference in the velocity between the streamline downstream of the combustion front and the one upstream, with the same result applying for the density distribution. This situation, together with the presence of pressure and density perturbations emanating from the detonation front, leads to the onset and sustainability of these instabilities and the formation of eddy structures. It is believe that such instabilities are Kelvin-Helmoltz instabilities, which are onset due to the difference in the velocity and density between the detonation products and the injected reactants. In addition to the gradients in velocity, the discontinuities are affected by the presence of numerical diffusion in the inviscid simulations.

After quantitative validation of the predicted temperature field and the characterization of the discontinuities and instabilities encountered in the 2D simualtions, it is possible to use the experimental data reported by [Liu et al., 2012] to further evaluate the 2D predictions. Therefore, the pressure history data provided in [Liu et al., 2012] is compared to the data from the pressure monitor points. The overall behavior of the passing wave is appropriately captured and both, the numerical solution and the experiments show the distinct pressure peaks due to the presence of a single detonation wave front. It is observed that the maximum pressure value is $10 \%$ under predicted by the numerical simulations. It is also observed that detonation wave products behind the detonation wave in the CFD simulations do not expand to values of pressure lower than $0.7 \mathrm{~atm}$. The experimental measurements of pressures lower than zero may have been caused due to the experimental measurement techniques, and there is no reason to expect static pressure measurements lower than the far field pressure during supersonic exhaust of the detonation products.

In addition to the quantitative comparison between the experimental measurements and the numerical solution, the pressure data permits the calculation of the detonation wave velocity inside the annular combustion chamber. The time elapsed between the two adjacent pressure peaks at the same monitor point is used to estimate the detonation wave velocity for each of the detonation wave cycles. The averaged detonation wave velocity at the stable periodic state from 


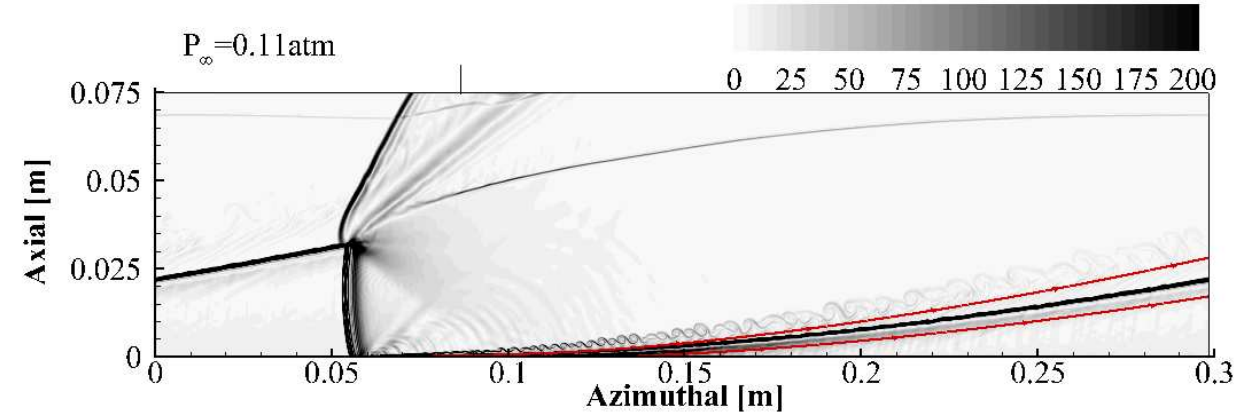

(a)

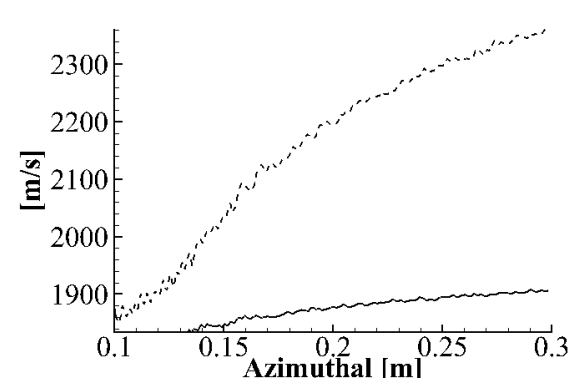

(b)

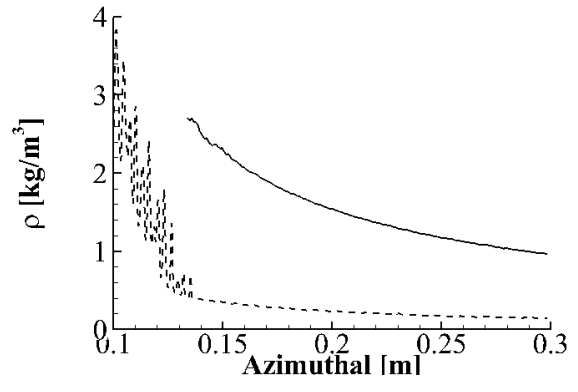

(c)

FIGURE 4.18: $2 \mathrm{D}\left|\frac{\partial \rho}{\partial \vec{x}}\right|$ contour with stream line locations (a), velocity (b) and density (b) distribution along streamlines. (Streamline downstream [- -] and upstream [-] of combustion front)

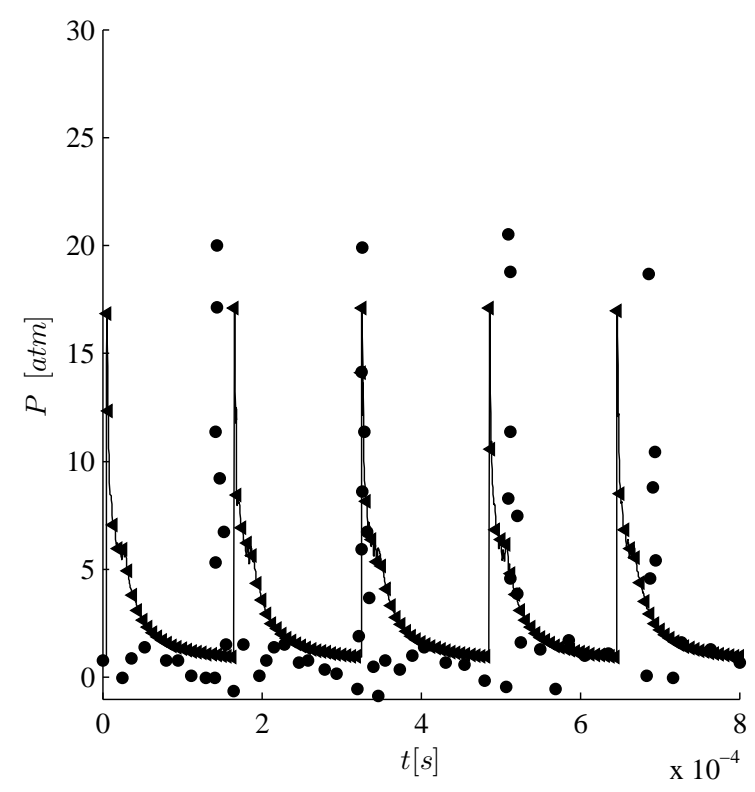

FIGURE 4.19: Comparison of pressure history between 2D CFD RDC simulation PBS with 1 step reaction model $(\triangleleft)$ and experiments ( 
the CFD predictions is equal to $1862 \frac{\mathrm{m}}{\mathrm{s}}$, compared to the experimental value reported in [Liu et al., 2012] $U_{D W, \exp }=1743 \frac{\mathrm{m}}{\mathrm{s}}$. It is then possible to conclude that the detonation wave velocity for 2D CFD simulations with the solver configuration of PBS-PISO-SQC-2UP and mesh size of $0.1 \mathrm{~mm}$, predicts the detonation wave velocity with a relative error of approximately $7 \%$. Considering the fact that the relative error calculated with the same solver configuration for a planar detonation wave was $0.6 \%$ (see Table 4.4 ), it is quite remarkable that the percent error in the detonation wave velocity prediction is in the single digits, despite the additional model simplifications. The predicted and experimental detonation wave velocity for RDC is lower than $U_{C J}$, and than the one predicted from the $2 \mathrm{D}$ detonation tube simulations. This is caused by the interaction of the detonation wave with the detonation products from the previous cycle. Such interaction produces the effect of a semi-unconfined detonation wave [Hishida et al., 2009]. The detonation wave velocity deficit present in RDC is off approximately $6 \%$ with respect to the confined detonation velocity, within the range reported by [Fujiwara and Tsuge, 1972].

In addition to the $2 \mathrm{D}$ results obtained using the PBS, numerical simulations were performed using the DBS while incorporating the single step an multi-step chemical models. It was observed that there is no significant difference for the simulations performed using the PBS or the DBS, with the 1 step reaction model. On the other hand, it is of major interest to analyze the differences due to the consideration of the multi-step reaction mechanism. For such purpose, the temperature contour for the 2D CFD simulation with the numerical configuration of DBS-AUSM-SQC-2UP are included in Figure 4.20. The results illustrated in Figure 4.20 were calculated on a computational mesh with $\delta x=0.25 \mathrm{~mm}$. Calculations with higher mesh resolutions were proven too costly and were not performed. Despite the coarser mesh, the 2D simulations appropriately predict the flow structure characteristic for RDC.

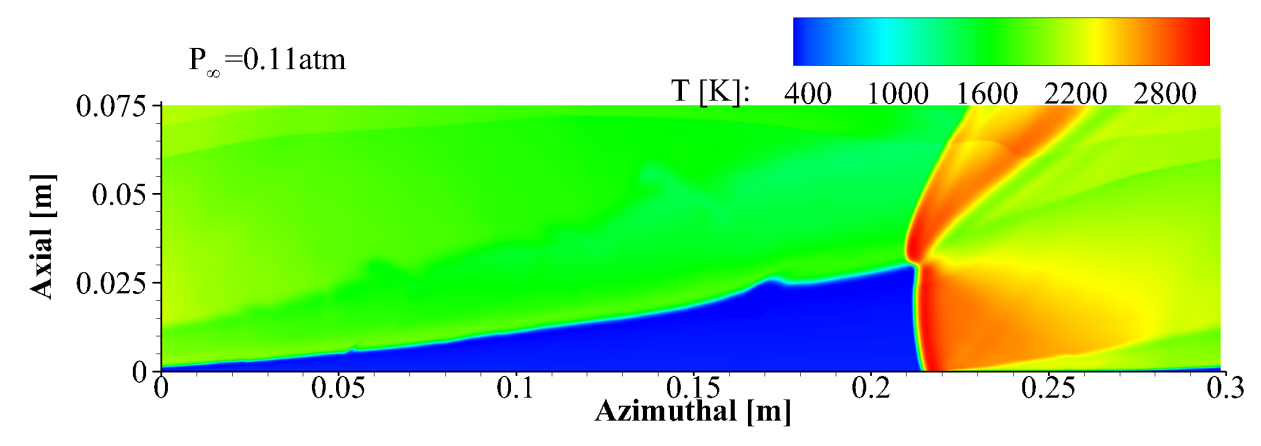

FIGURE 4.20: 2D temperature contour for DBS-AUSM-SQC-2UP RDC and multi step chemistry prediction for model validation

Similarly to what was reported for the solution using PBS, the contour of the magnitude of the density gradient is given in Figure 4.21. The high density gradient regions depict the flow discontinuities characteristics of RDC. Due to the larger mesh size of the DBS simulation, the resolution of small scale features such as the eddies near the combustion front are not captured. 
However, the presence of the oblique pressure waves is still resolved, along with the detonation front, and the combustion front.

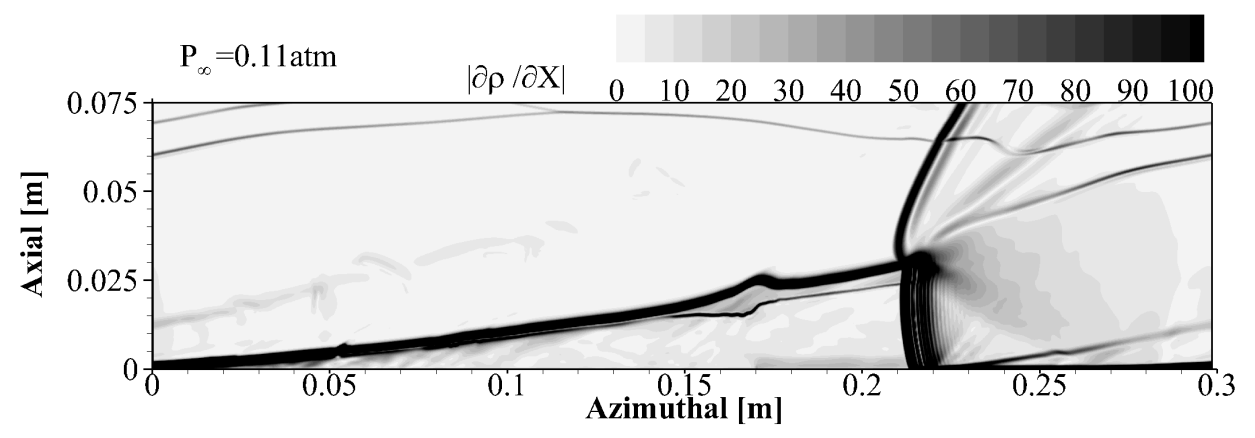

FIGURE 4.21: 2D $\left|\frac{\partial \rho}{\partial \vec{x}}\right|$ contour for DBS and multi step chemistry

The pressure history was also sampled for the set of simulations using the DBS and the multi-step reaction mechanism. The pressure history from the 2D CFD simulations was then compared with the experimental data. The comparison shows that the presence of a distinct detonation wave is appropriately captured (Figure 4.22). Furthermore, the maximum pressure value of the pressure behind the detonation wave is of equal to 20atm, leading to a relative error with respect to the experimental data of only $3 \%$. Additionally, the expansion of the detonation products behind the detonation wave follows the expected behavior, where the minimum static pressure of the expanding detonation products is not lower than 0.9atm. In addition to the comparison between experimental and CFD prediction, the pressure history was also used to calculate the detonation wave velocity inside the combustion chamber. The predicted detonation wave velocity for the DBS and multi-step mechanism was of $1831 \frac{\mathrm{m}}{\mathrm{s}}$, meaning that the relative error with respect to the experimental detonation wave velocity is of only 5\%. Taking into account that for the same computational settings the error in the prediction of the propagation velocity was of $0.2 \%$, but that for coarser meshes it could be of up to $2.3 \%$, it is possible to conclude that the prediction of the detonation wave velocity in the annular chamber with the multi-step reaction mechanism is appropriately predicted. Once more, it is quite remarkable that despite of the additional modeling assumptions like domain simplifiaction, injection system model and neglected diffusive terms; the relative error does not increase substantially.

It is then of great interest to evaluate the distribution of major and pollutant species in the combustion chamber of RDC. For such purpose, the contours of $\mathrm{H}_{2}$ mass fraction, $\mathrm{OH}$ mass fraction and corrected dry NOx concentration are provided in Figure 4.23. The mass fraction contour for $\mathrm{H}_{2}$ shows the characteristic wedged shape unreacted region. It is observed that outside this region, almost all the fuel $\left(\mathrm{H}_{2}\right)$ is consumed except by a strip region that forms from the upper corner of the detonation front. The $\mathrm{OH}$ contour plot can also be used to determine the existence of the characteristic flow structures in RDC. The $\mathrm{OH}$ contour plots are often correlated with the chemiluminescence photographs for RDC. It is then possible to state that three distinct 


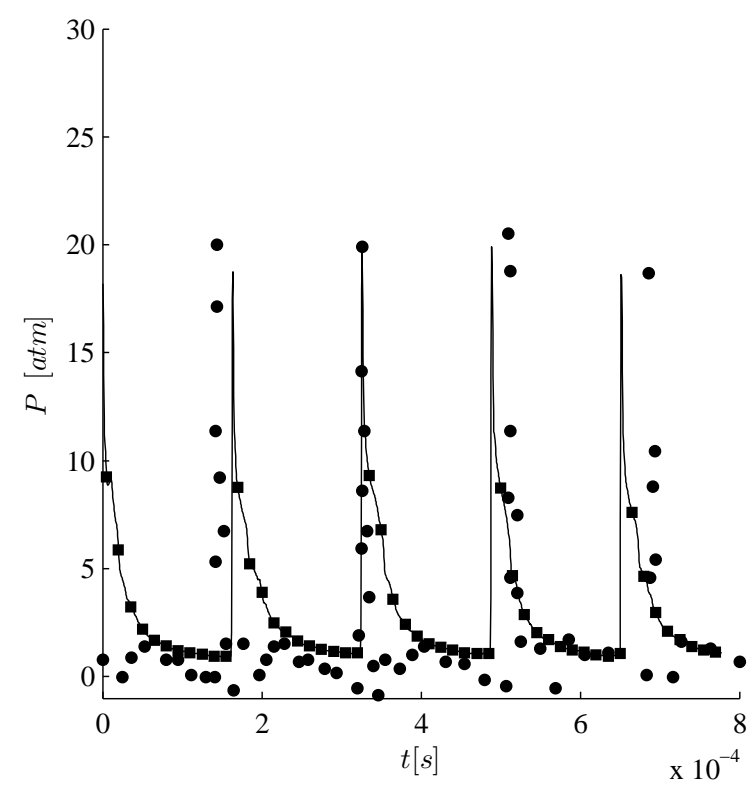

FIGURE 4.22: Comparison of pressure history between 2D CFD RDC simulation with the DBS and multi step reaction mechanism ( $\mathbf{\square})$ and experiments (

reaction regions form in RDC: the detonation front, in which most of the mass of the reactants is consumed, the deflagration or flame front, and the region formed in the upper corner of the detonation front. In this smaller reaction front, unreacted fuel from the fresh mixture section and partially reacted gases from the deflagration front go through a shock wave; the strength of such shock wave is not as large as the detonation front. Hence, the gases do not fully react in the viscinity of this shock wave front, but are influenced by the detonation products downstream of the oblique shock wave. One of the advantages of using a multi-step reaction mechanism is the possibility to study the pollutant concentration in RDC. The contour of dry $15 \% \mathrm{O}_{2}$ corrected $\mathrm{NO}$ concentration is illustrated in Figure 4.23(c). It is observed that high NO concentrations form in the expanding detonation products region, and that a lower concentration region is formed from the combustion products produced in the deflagration zone.

In addition to the NO distribution in the domain, the mass weighted average of the dry corrected NO concentration at the combustion outlet was calculated as a function of time. Results show that the average $\mathrm{NO}_{d r y, c o r r} 15 \% \mathrm{O}_{2}$ is $164 \mathrm{ppm}$, also during one cycle of the detonation wave the oscillations of the NO concentration did not exceed $10 \mathrm{ppm}$. It is important to highlight that the predicted $\mathrm{NO}$ concentration corresponds to stoichiometric mixture of $\mathrm{H}_{2}$-Air.

In order to evaluate the influence of equivalence ratio on nitrogen monoxide (NO) emission for $\mathrm{RDC}$ of $\mathrm{H}_{2}$-Air,an additional set of two dimensional simulations were performed. The injection boundary conditions were modified in order to simulate RDC with a global equivalence ratio $(\Phi)$ of 0.5 . The inlet mass flow rate was imposed in order to match the one corresponding to the $\Phi=1.0$ base case. Due to the change in the mixture composition, the stagnation pressure at the injection manifold $\left(P_{o, \text { inlet }}\right)$ was slightly decreased to $10.27 \mathrm{~atm}$, while the stagnation 


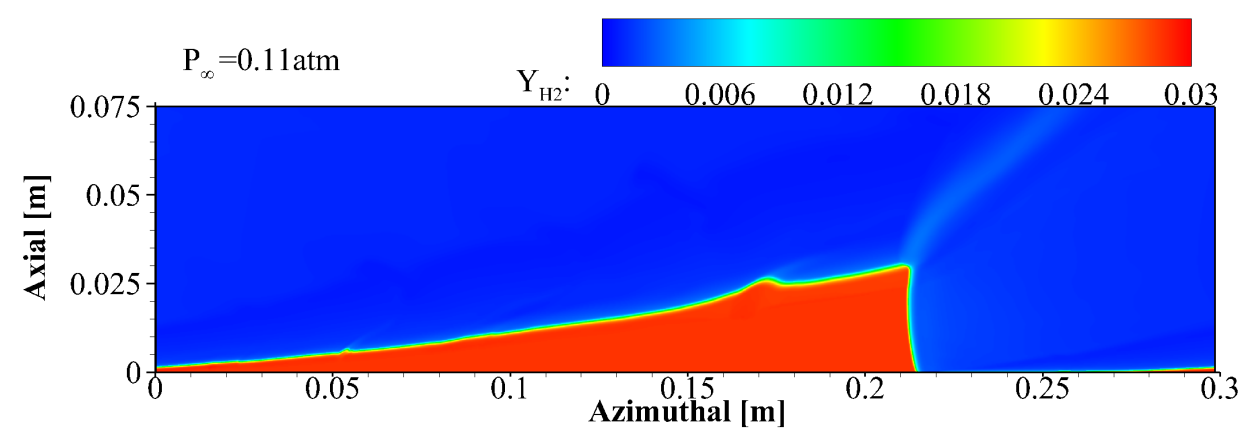

(a)

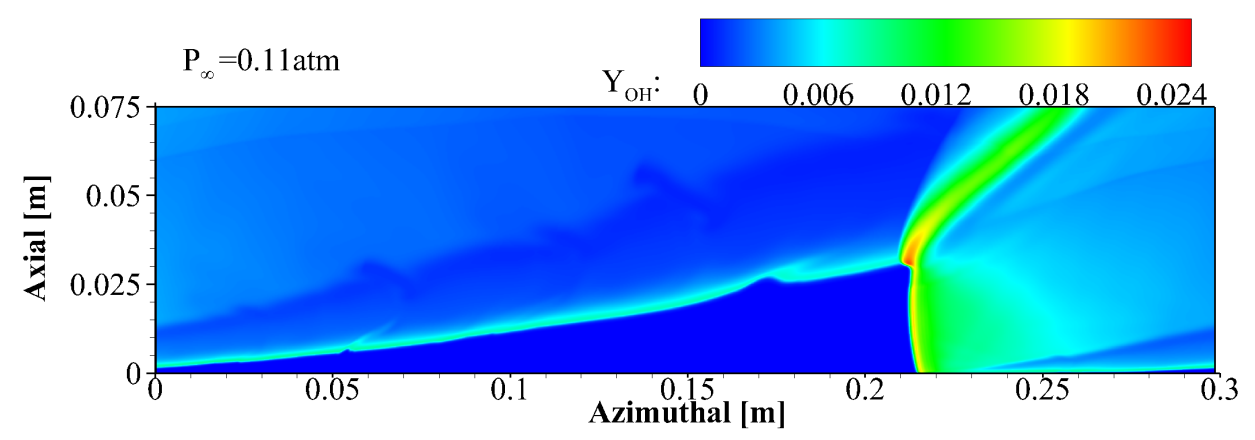

(b)

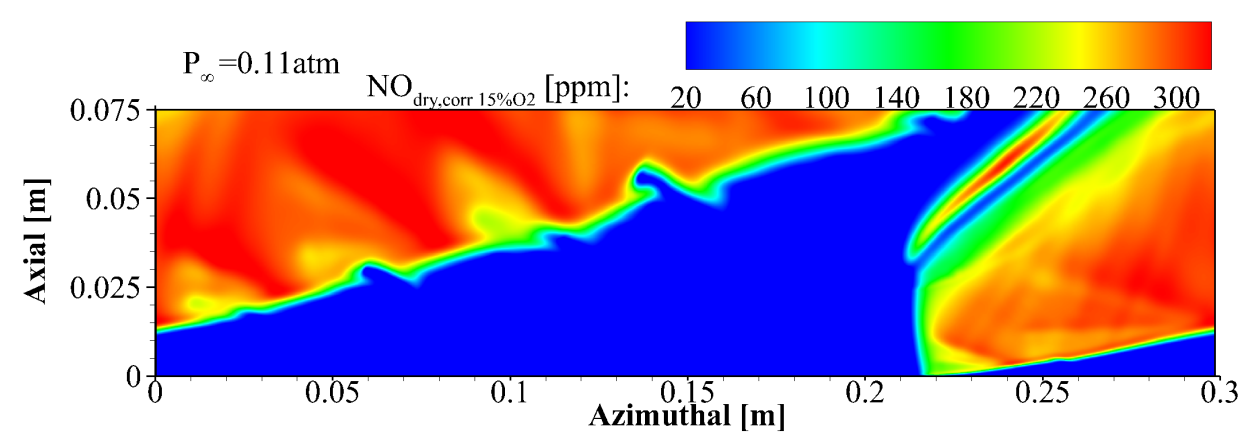

(c)

FIGURE 4.23: $2 \mathrm{D}$ contour for major species and pollutant concentrations in RDC. $Y_{\mathrm{H}_{2}}$ (a), $Y_{\mathrm{OH}}$ (b) and $\mathrm{NO}_{\text {dry, } \text {,orr } 15 \% \mathrm{O}_{2}}(\mathrm{c})$

temperature remained unchanged and equal to $300 \mathrm{~K}$. The composition of the reactant was set according to an equivalence ratio of 0.5 for $\mathrm{H}_{2}$-Air combustion. Continuous detonation was predicted under fuel lean conditions and the predicted detonation wave velocity was equal to $1563 \frac{\mathrm{m}}{\mathrm{s}}, 16 \%$ lower than that for $\Phi=1.0$. The solution of the $2 \mathrm{D}$ simulation is illustrated as temperature, and species concentrations contours in Figure 4.24.

As depicted in Figure 4.24(a), the overall temperature in the computational domain decreases with the decrease in equivalence ratio (see Figure 4.16. In fact, the averaged total temperature at the outlet of the combustion chamber for $\Phi=0.5$ is equal to $1665 \mathrm{~K}, 7.2 \%$ lower than the one under stoichiometric conditions. Similarly, the averaged stagnation pressure 


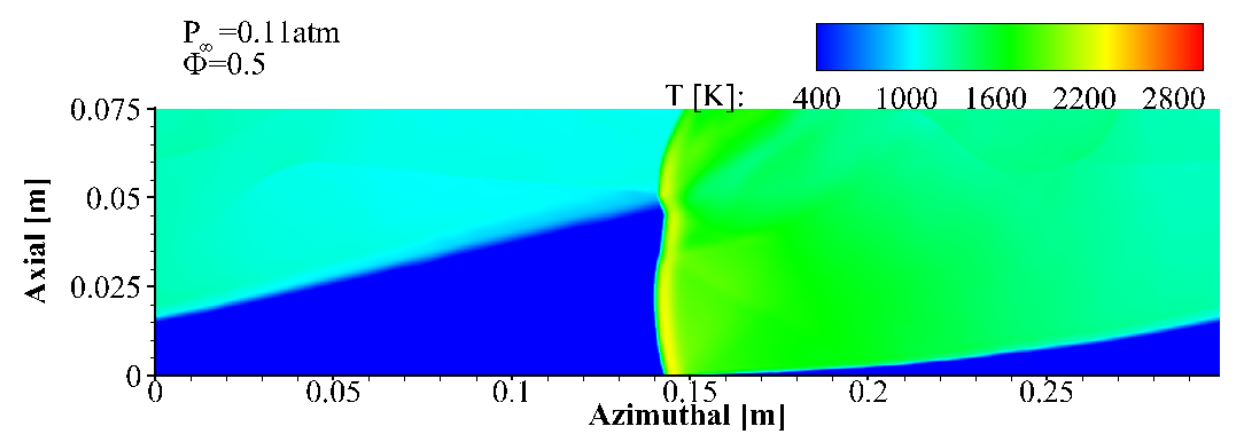

(a)

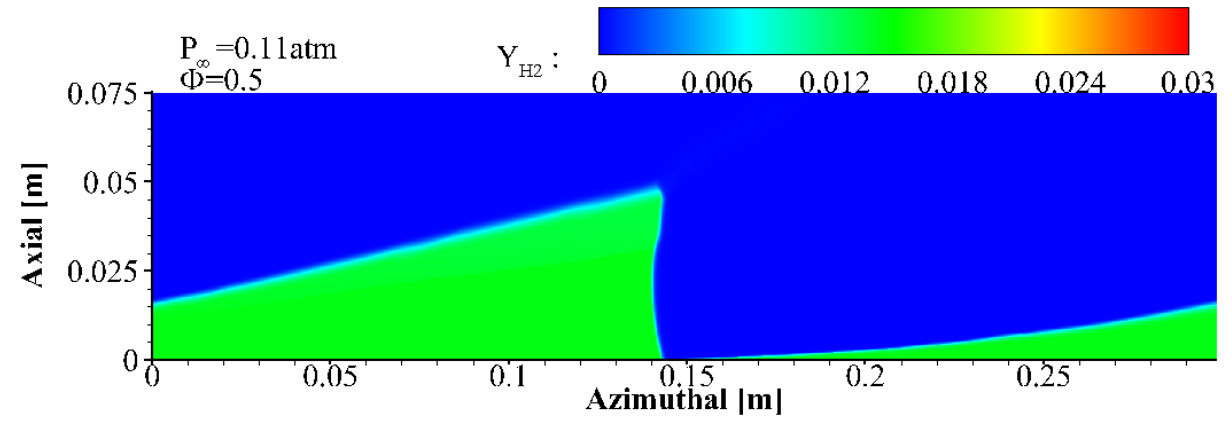

(b)

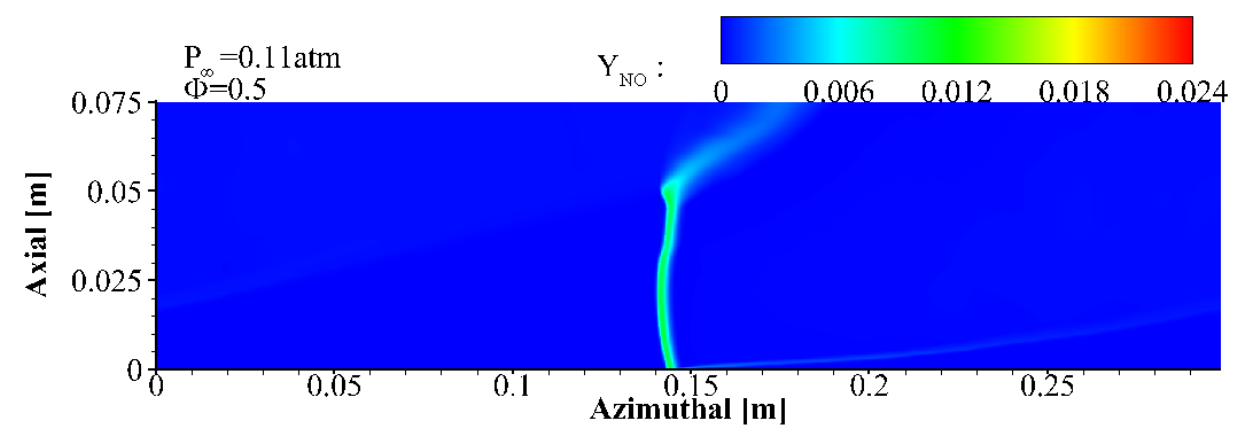

(c)

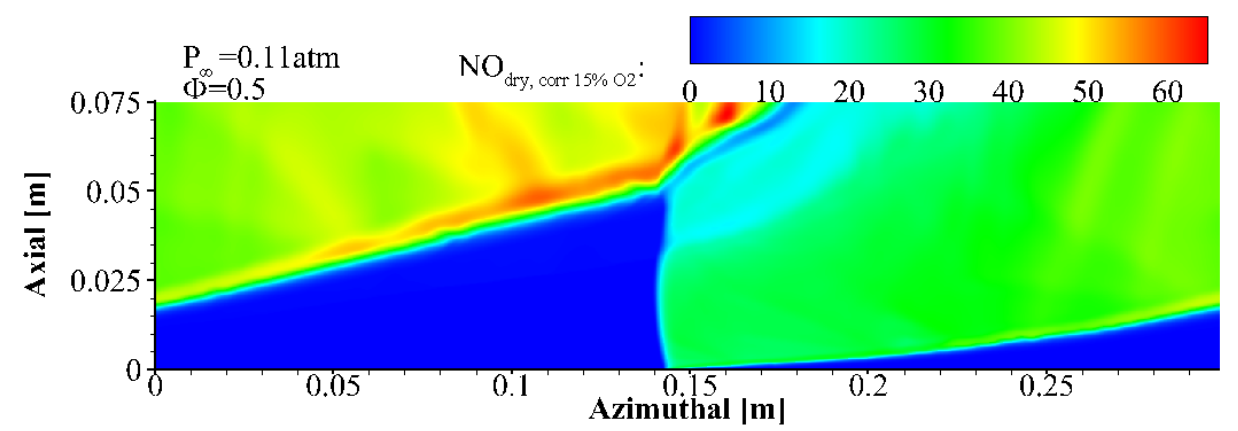

(d)

FIGURE 4.24: Two dimensional contours of RDC simulation with multi step reaction mechanism and $\Phi=0.5$. Temperature (a), $\mathrm{H}_{2}$ mass fraction (b), $\mathrm{OH}$ mass fraction (c), and $\mathrm{NO}_{d r y, c o r r} 15 \% \mathrm{O}_{2}$ 
at the outlet $\left(P_{o, \text { outlet }}\right)$ decreased and is equal to $1.94 \mathrm{~atm}$, a $24.5 \%$ reduction with respect to stoichiometric conditions.

In addition to the differences in the temperature contours, differences in the $\mathrm{H}_{2}$ mass fraction distribution in the computational domain were observed. The $\mathrm{H}_{2}$ mass fraction distribution given in Figure 4.24(b) shows that, in contrast to the solution for $\Phi=1.0$ (see Figure 4.24(a)), the $\mathrm{H}_{2}$ is consummed by the detonation front in its totality and there is no presence of unreacted fuel expanding from the upper edge of the detonation front. Moreover, the concentration of the hydroxide radical $(\mathrm{OH})$ decreases specially in the deflagration front region, see Figure 4.24(c).

More importantly, a significant change in the nitrogen oxide concentration in the computational domain was obtained. The corrected NO concentration contours illustrated in Figure 4.24(d), show a significant reduction when compared with the results for $\Phi=1.0$. The predicted mass weighted averaged for the corrected Nitrogen Monoxide concentration at the combustion chamber outlet with $\Phi=0.5$ is equal to $34.63 \mathrm{ppm}$. Based on such predictions it is possible to conclude that Nitrogen Monoxide emissions are reduced by approximately $79 \%$ for $\mathrm{H}_{2}$-Air RDC when the equivalence ratio is reduced from 1.0 to 0.5 .

\subsubsection{Three Dimensional Simulations of RDC for Model Validation}

After concluding that the simulations performed under the assumption of a simplified 2D domain appropriately predicted the main flow structures for RDC, it is important to evaluate the influence of 3D effects on RDC predictions. This subsection covers the influence of 3D parameters in the prediction of RDC in such a manner that the level of complexity increases gradually. Consequently, the results for 3D simulations without the injection section and under the assumption of neglectable viscous effects are presented first. These results are followed by the simulations performed in the simplified 3D domain while considering the influence of viscous term and mass diffusion, but under the assumption of adiabatic wall boundaries. Next, the influence of heat transfer through the wall is evaluated under two different boundary conditions. Finally, the simulations of the 3D domain including the injection section are presented.

The effects of walls in RDC operation can be separated in two. One is the influence of the curvature and the second is the viscous and heat transfer effects at the wall. The set of simulations presented were intended to assess the former. For this set of simulations the 3D simplified domain is used; where the injection section is not included in the computational mesh and instead is calculated using the injection model explained in Section 3.4. The simplified three dimensional domain was partitioned into a computational mesh with average cell size $(\delta x)$ of $0.5 \mathrm{~mm}$. The use of a smaller cell size for 3D simulation increases the computational cost, and it was deemed unpractical for the computational resources available for the current study. Despite 
the coarser mesh size, the detonation wave and related flow structures are appropriately captured. Additionally, the knowledge from the 2D planar detonation wave simulations permits a clear assessment of the predictions performed with lower mesh resolutions. The 3D simulations with neglected viscous effects were performed for the 1 step and multiple step reaction models. As presented in Figure 4.25, the temperature and pressure contours for the 3D simulations using both chemical models appropriately predict the flow structure in RDC and no significant differences in the main flow structures between the $2 \mathrm{D}$ and the 3D prediction were observed.
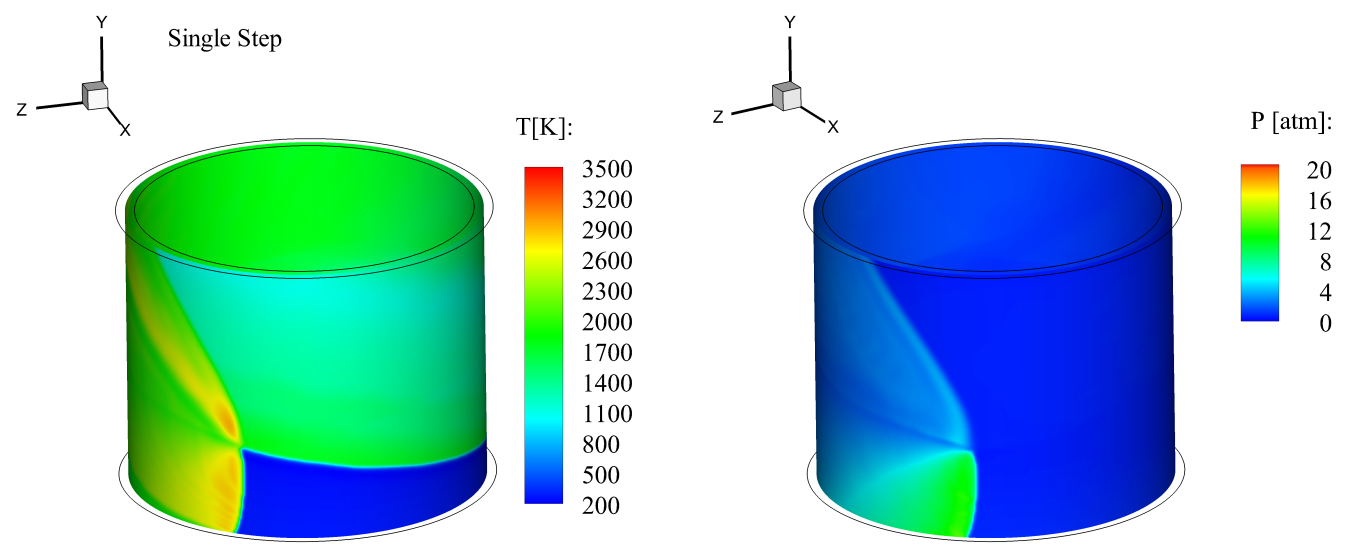

(a)
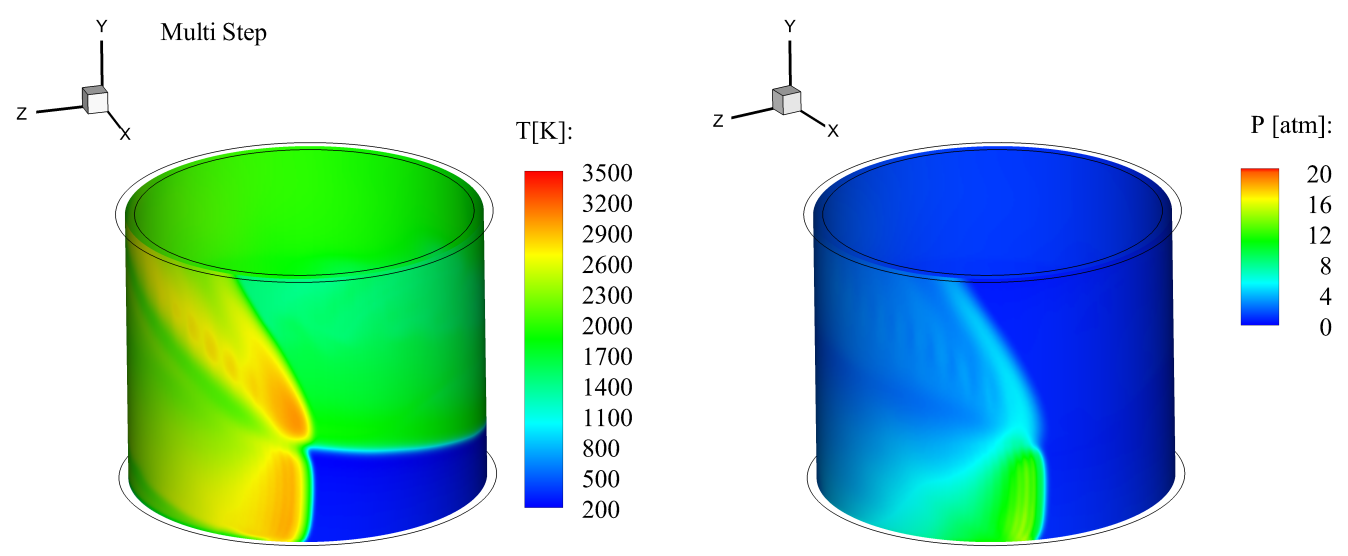

(b)

FIGURE 4.25: 3D temperature and pressure contour for 1 step (a) and multi step (b) chemistry model

The contours shown in Figure 4.25 are taken at the mid annular surface of the combustion chamber; therefore no 3D radial structures were captured. However, by analyzing the temperature and pressure distribution in the cross section of the chamber some radial features are observed. The effect of the curvature in the pressure field is illustrated in Figure 4.26(a), 


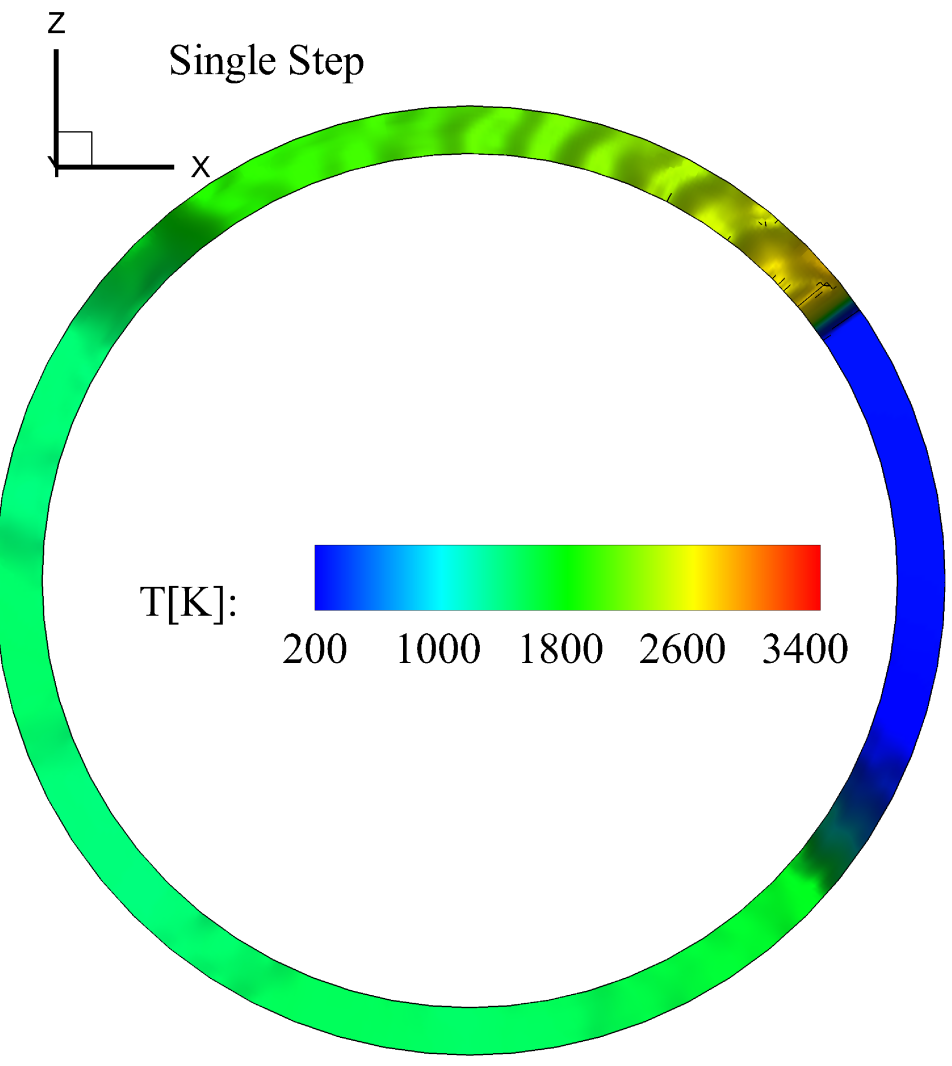

(a)

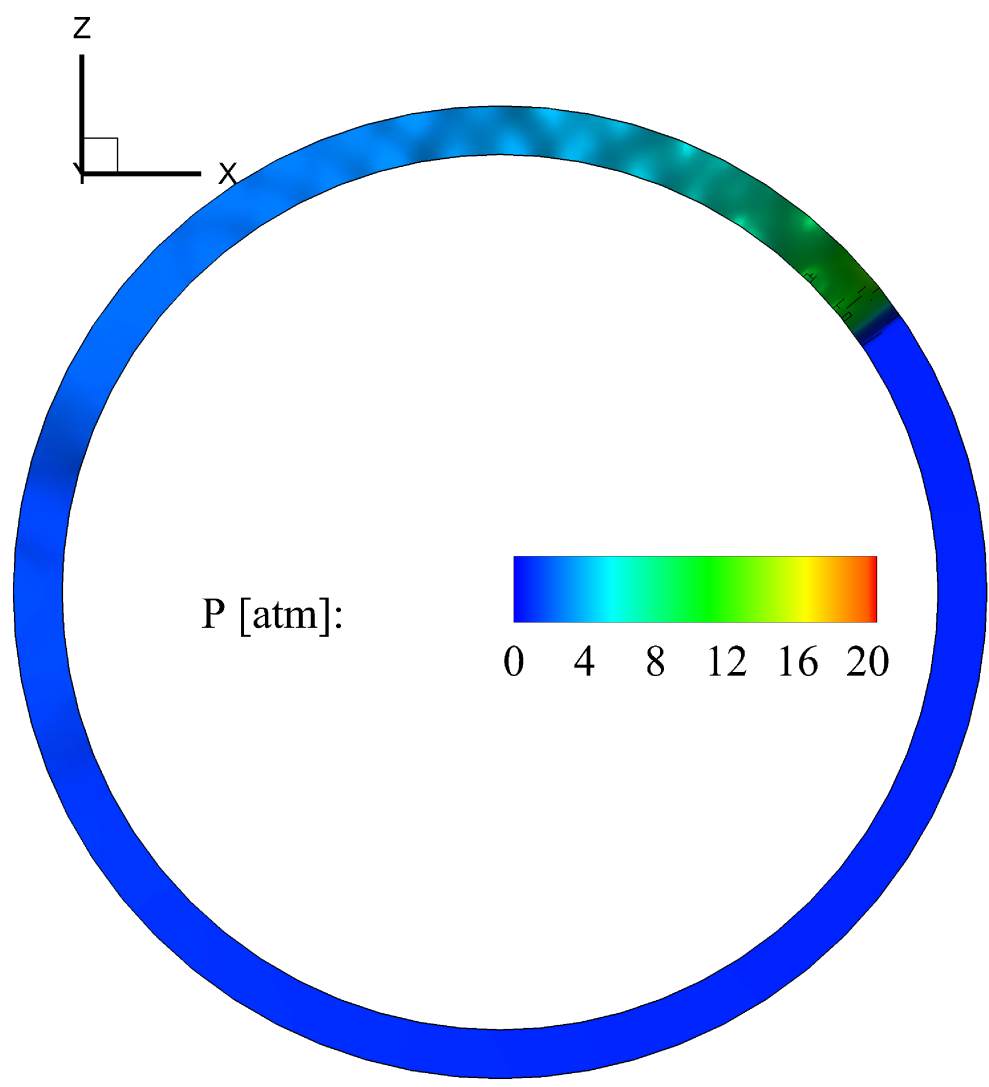

(b)

Figure 4.26: Cross sectional temperature (a) and pressure (b) contours from 3D simulation at $15 \mathrm{~mm}$ from inlet surface. 
which shows a contour plot of the pressure at the axial distance of $15 \mathrm{~mm}$. It is observed that radial variations of the temperature are more prevalent near the detonation front. These fluctuations are correlated with the presence of pressure waves that reflect from the inner and the outer wall as the detonation products expand.

Monitor points were also used in the 3D simulations to evaluate the pressure history inside the annular combustion chamber. The results show that appropriate prediction of the pressure wave history behind the detonation wave was obtained. Figure 4.27 illustrates the results obtained for the 1 step and multi-step reaction mechanism, compared to the experimental data. The radial fluctuations in pressure do not appear in the monitor pressure signal. This can be attributed to the fact that the monitor point was located $2 \mathrm{~mm}$ away from the inlet, while the pressure fluctuation shown in Figure 4.26 are $15 \mathrm{~mm}$ away from the inlet plane. The pressure distribution from the pressure probes was then used to calculated the average detonation wave velocity in the mid plane of the annular chamber. The calculated average detonation wave velocity for the simulation with the 1 step reaction mechanism was equal to $1848 \frac{\mathrm{m}}{\mathrm{s}}$ and for he multi-step chemical model was equal to $1724 \frac{\mathrm{m}}{\mathrm{s}}$, leading to a relative error with respect to experimental data of $6.0 \%$ and $1.0 \%$ respectively. 


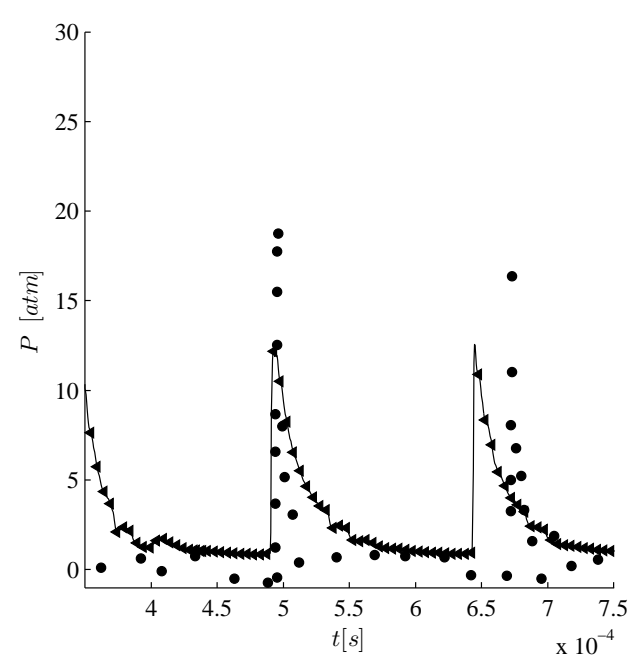

(a)

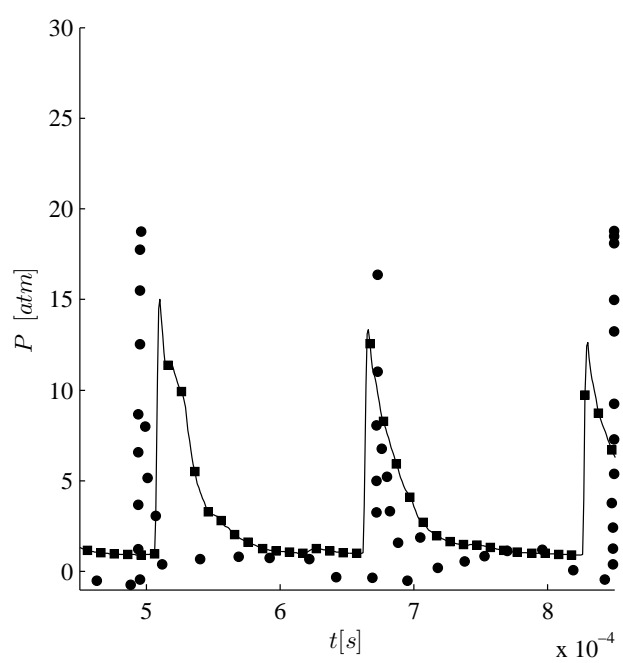

(b)

FIGURE 4.27: Comparison of pressure history between 3D CFD RDC simulation and experiments. Predictions with 1 step reaction model [ 4$]$ and experiments ( $)$ (a) and with multi step reaction model $[\mathbf{\square}]$ and experiments

Based on the results initially discussed in this subsection, it is possible to conclude that 3D simulations of RDC under the assumption of neglectable viscous effects appropriately predict the velocity of the detonation wave inside the annular chamber. It was also observed that $3 \mathrm{D}$ effects appear in the radial direction of the annular chamber due to the curvature between the inner and the outer cylinder. Since the current set up has a mean diameter to annular gap ratio $\left(\frac{D_{\text {mean }}}{\Delta_{c}}\right)$ of 20 , these effects did not yield to a dramatic difference in the flow structures; however, the velocity of the detonation wave was consistently reduced from the $2 \mathrm{D}$ to the $3 \mathrm{D}$ simulations. A summary of the predicted detonation wave velocity and the relative error with the experimental value are given in Table 4.6.

TABLE 4.6: Summary of detonation wave velocity prediction for model validation

\begin{tabular}{cccccccc}
\hline \hline Domain & Solver Configuration & Chem. Model & $\delta x$ & $\begin{array}{c}U_{D W} \\
\mathrm{~m}\end{array}$ & $\begin{array}{c}E_{U} \\
\mathrm{~m} / \mathrm{s}\end{array}$ & $\begin{array}{c}P_{o, \text { outlet }} \\
\mathrm{atm}\end{array}$ & $\begin{array}{c}T_{o, \text { outlet }} \\
\mathrm{K}\end{array}$ \\
\hline 2D & PBS-PISO-SQC-2UP & 1 Step & 0.10 & 1862 & 7.0 & 2.57 & 1794 \\
2D & DBS-AUSM-SQC-2UP & Multi step & 0.25 & 1831 & 5.0 & 2.80 & 2222 \\
3D & PBS-PISO-SQC-2UP & 1 Step & 0.25 & 1848 & 6.0 & 2.63 & 1885 \\
3D & DBS-AUSM-SQC-2UP & Multi step & 0.25 & 1724 & 1.0 & 2.95 & 2237 \\
\hline$U_{D W, \exp }=1743 \frac{m}{s}$ & & & & & & \\
\hline
\end{tabular}

Based on the results presented in Table 4.6, it is opossible to establish that for 2D and 3D simulations the multistep reaction mechanism predicts a lower detonation wave velocity, which is closer to the experimental value. Such behavior was also evidenced in: Table 4.5 from the planar detonation wave predictions, and to a lower degree in Table 4.2 in the predicted CJ detonation wave velocity. Recapitulating such evidence and together with the predicted ZND structure provided in Figure 4.8, it is possible to conclude that the single step reaction 
mechanism predicts a stronger detonation wave than that predicted by the multi step reaction model. This conclusion is supported by the fact that the predicted ZND and planar detonation wave structure with the 1 step reaction mechanism shows a more intense heat release distribution in the detonation wave structure. The reduction of the detonation wave velocity obtained from the $3 \mathrm{D}$ inviscid simulation shows that by considering the curvature effects, leads to a better prediction of the detonation wave velocity.

To further analyze the effects of curvature in RDC peformance, 3D simulations for two additional combustion chamber diameters were performed. The geometry of the combustion chamber was modified by changing the diameter of the inner cylinder $\left(D_{i n}\right)$ to $95 \mathrm{~mm}$ and 80 $\mathrm{mm}$, resulting in $\frac{D_{\text {mean }}}{\Delta_{c}}$ ratio of 40 and 10 , respectively. While the geometry was modified, the injection stagnation pressure $\left(P_{o, \text { man }}\right)$ was maintained constant. Despite the fact that the mass flow rate at the throat of the injection section under shocked conditions remained constant (see Equation 3.12), the mass flux at the combustion chamber changed due to the alteration in its cross sectional area.

The first result to be analyzed is the combustion chamber with inner diameter of $95 \mathrm{~mm}$ $\left(\frac{D_{\text {mean }}}{\Delta_{c}}=40\right)$. For this geometric configuration, instabilities in the deflagration front were observed. As illustrated by the temperature contour in Figure 4.28, the reaction front is strongly corrugated under the increased mass flux. It is believed that since the mass flux is increased in the narrower annular section, the velocity of the reactant mixture increases. Such a situation leads to an onset of combustion instabilities in the deflagration front. Eventually these fluctuations create pockets of unreacted or partially reacted gases among the detonation products, which are either consumed by the oblique shock wave or leave the combustion chamber before complete reaction. If the unreacted mixture is consumed by the shock wave, strong pressure waves are generated in the combustion chamber. These pressure disturbances travel upstream towards the inlet surface and influence the local injection velocity of fuel and oxidizer mixture. The local variation of injection velocity, pressure and temperature generate further corrugation of the deflagration front. Hence, it is possible to conclude that the unstable regime encountered under the increased mass flux condition is self sustaining. Figure 4.28(b) shows a pressure gradient plot, where the waves behind the detonation wave are evident.

In the case of the numerical simulation for 3D RDC with $D_{\text {in }}=80 \mathrm{~mm}\left(\frac{D_{\text {mean }}}{\Delta_{c}}=10\right)$, no fluctuations in the deflagration front were observed. However stronger presence of the radial variations in pressure and temperature were predicted. Figure 4.29 shows the contours of temperature and pressure in a cross sectional view at $15 \mathrm{~mm}$ from the inlet. When compared to the structure in Figure 4.26 for the annular chamber with $D_{i n}=90 \mathrm{~mm}$, it is observed that the radial variation of temperature and pressure are not only stronger, but they also extend further in the azimuthal direction. Additionally, it is observed that the maximum of static pressure value is present in the outer portion behind the detonation front. 


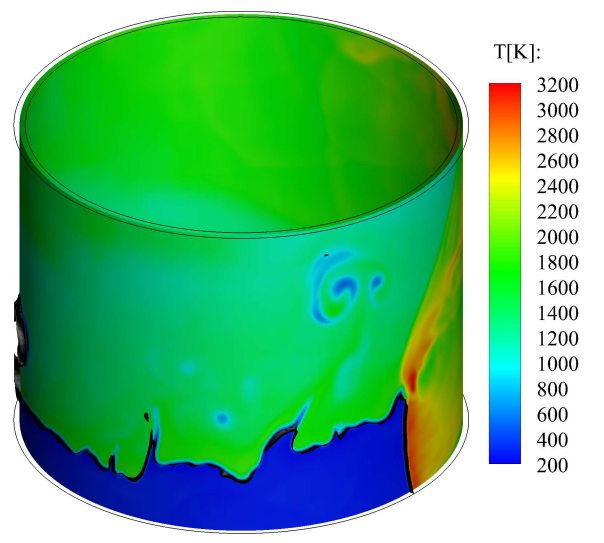

(a)

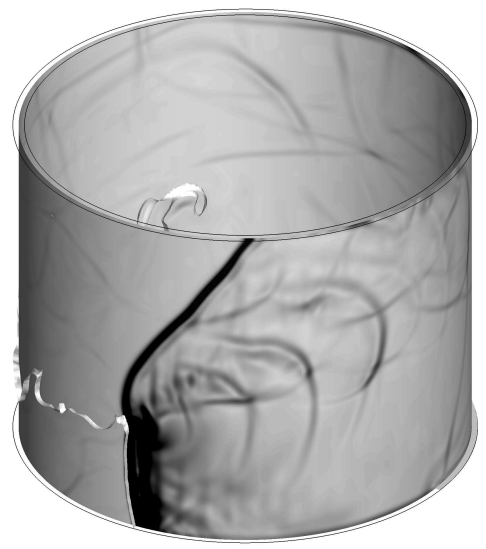

(b)

FIGURE 4.28: Temperature (a) and pressure gradient (b) contours for 3D RDC with $D_{\text {in }}=95 \mathrm{~mm}$

In addition to the illustration of the presence of pressure waves in the radial direction of the annular RDC chamber, it is of great value to quantify the pressure and temperature variation in the radial direction for RDC in annular chambers of different inner diameters. To do so, the instantaneous temperature and pressure distribution ahead of the detonation wave and behind of the detonation front, taken at $15 \mathrm{~mm}$ from the inlet plane are presented in Figure 4.30(a,b) and (c,d) for $D_{i n}=90 \mathrm{~mm}$ and $D_{i n}=80 \mathrm{~mm}$, respectively. It is observed that for both diameters the variation of pressure and temperature in the radial direction in front of the detonation wave is not significant (see Figure 4.30(a and c)). On the other hand, radial gradients of pressure and temperature are evident behind the detonation front (see Figure 4.30(b and d)). The predicted instantaneous temperature gradient in the radial directions are equal to $1039 \mathrm{~K} / \mathrm{m}$ and $4620 \mathrm{~K} / \mathrm{m}$ for $D_{\text {in }}=90 \mathrm{~mm}$ and $80 \mathrm{~mm}$, respectively. It is observed that for $D_{i n}=80 \mathrm{~mm}$, the temperature near the inner diameter decreases substantially. Such decrease in the temperature can be related to the expansion of the detonation products behind the detonation wave that is enhanced by the curvature of the inner diameter of the combustion chamber. It is expected that a stronger expansion occurs close to the inner wall of the combustion chambers since the surface of the inner cylinder diverges away from the direction of the flow. In regards to the pressure distribution, it is observed that the gases expand to a lower pressure for the combustion chamber with $D_{\text {in }}=80 \mathrm{~mm}$. Such lower pressure is explained due to the increases expansion area of the combustion chamber $\left(A_{t} / A_{e}=0.2\right.$ vs $\left.A_{t} / A_{e}=0.08\right)$, the injection pressure of the fresh reactants decreases as the expansion area of the injection section increases. In addition to the general decrease of the static pressure with the decrease of inner diameter, it is also observed in Figure 4.30(d) that significantly higher pressures are obtained near the surface of the outer cylinder. In the case of $D_{\text {in }}=90 \mathrm{~mm}$, the instantaneous pressure difference between the inner and outer diameter of the combustion chamber for the sampled line is equal to $1.88 \mathrm{~atm}$, and $3.9 \mathrm{~atm}$ for the $D_{i n}=80 \mathrm{~mm}$ geometry. Furthermore, it was obtained that the gradient of pressure 


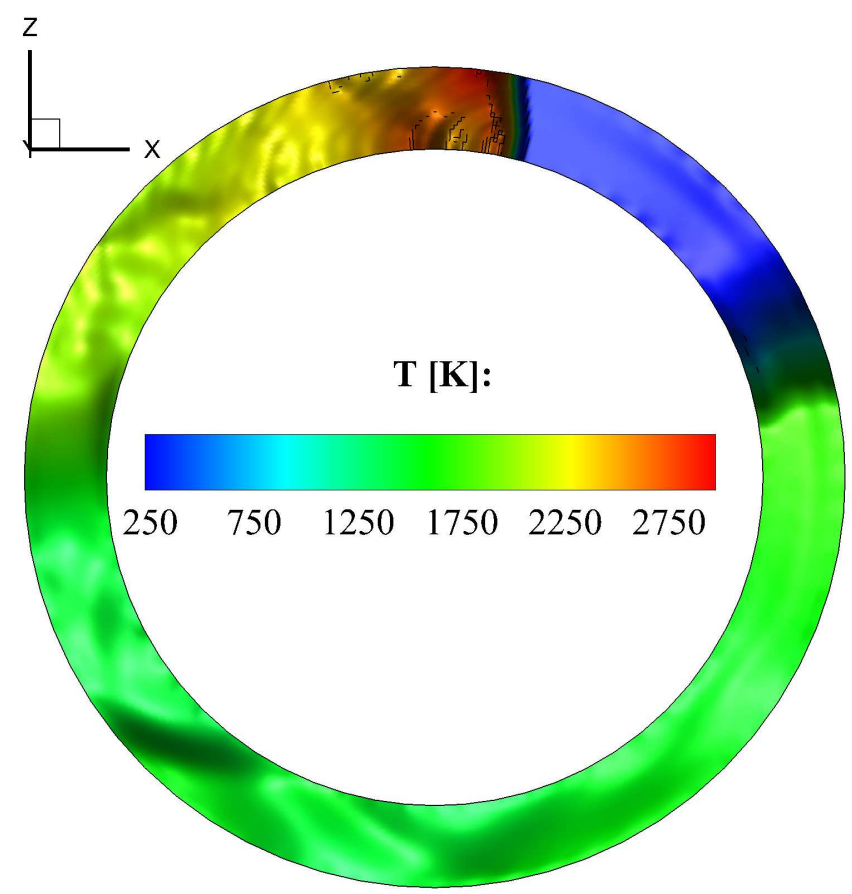

(a)

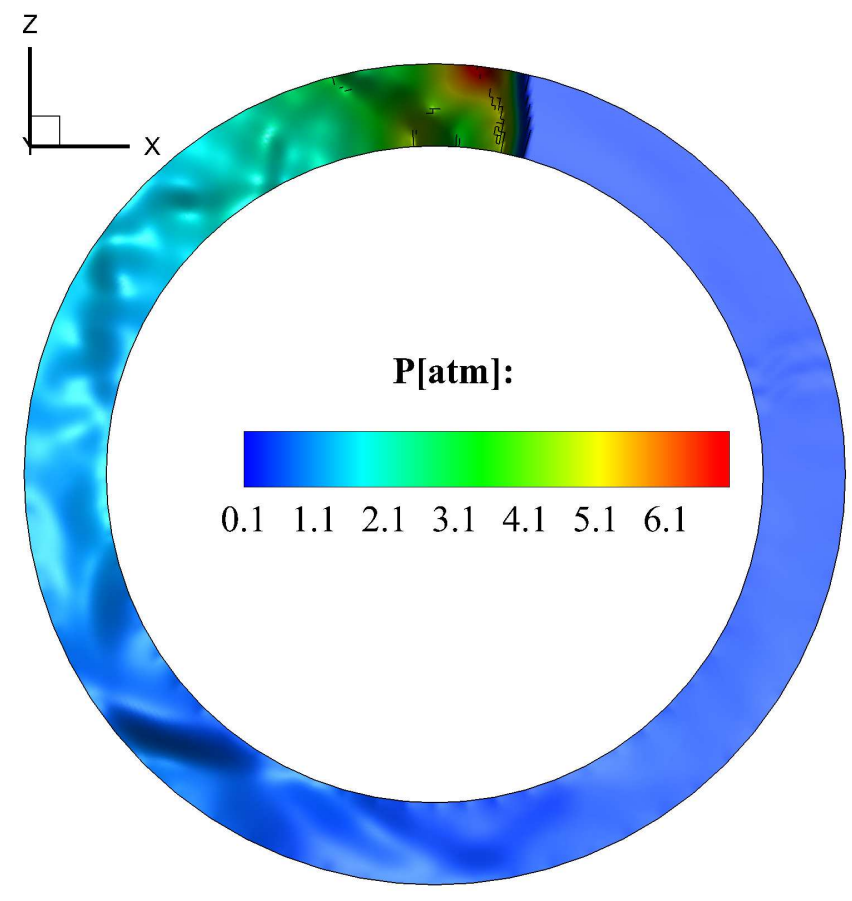

(b)

FIGURE 4.29: Cross sectional temperature (a) and pressure (b) contours from 3D inviscid simulations at $15 \mathrm{~mm}$ from inlet surface for RDC chamber with $D_{\text {in }}=80 \mathrm{~mm}$. 
in the radial direction behind the detonation wave for inner diameter of $90 \mathrm{~mm}$ and $80 \mathrm{~mm}$ are equal to $397 \mathrm{~atm} / \mathrm{m}$ and $400 \mathrm{~atm} / \mathrm{m}$, respectively.

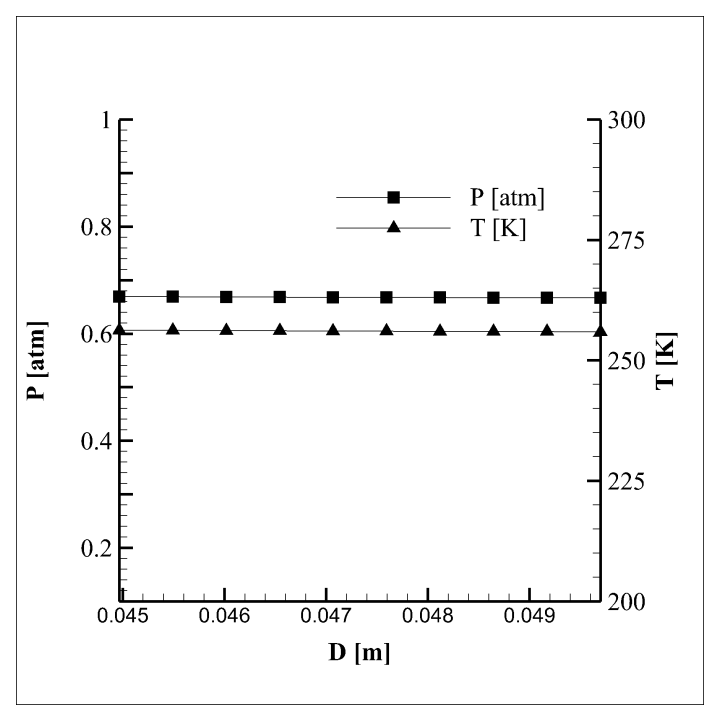

(a)

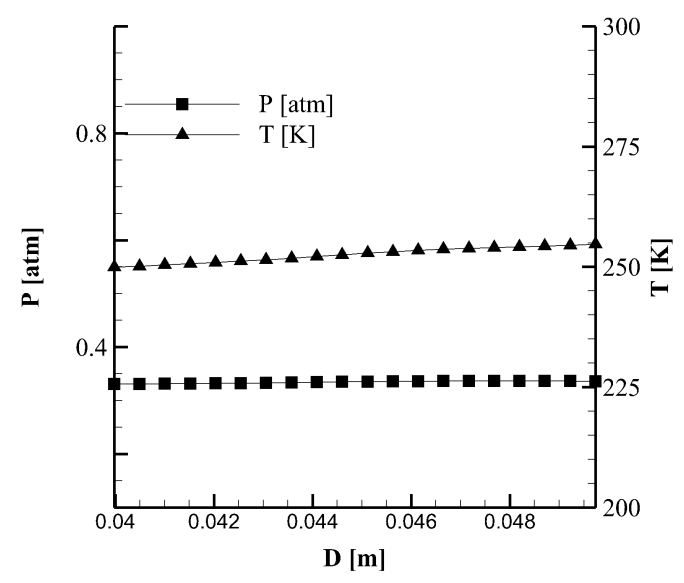

(c)

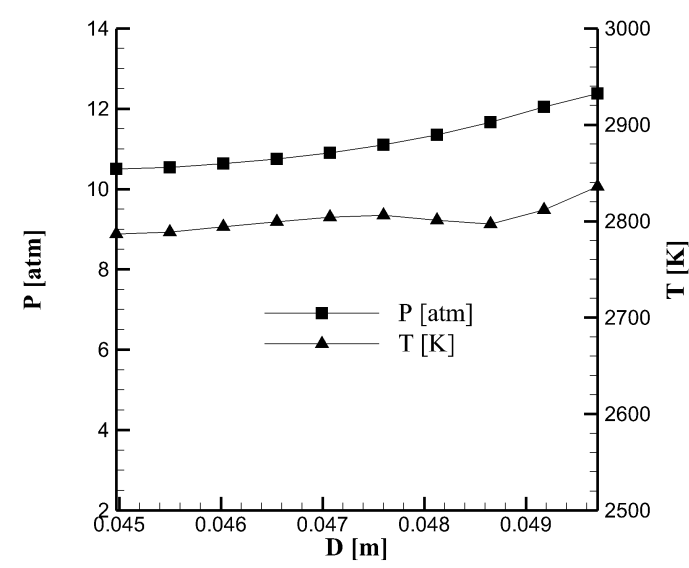

(b)

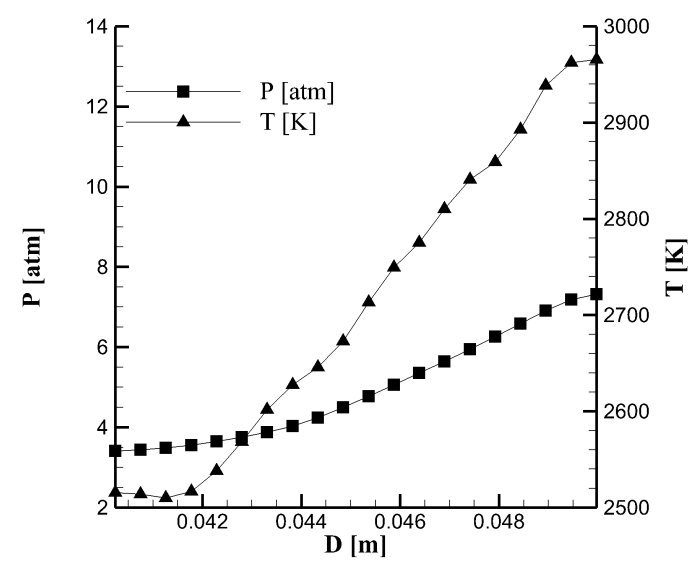

(d)

FIGURE 4.30: Radial distribution of temperature and pressure for RDC camber with $D_{\text {in }}=95 \mathrm{~mm}$ and $80 \mathrm{~mm}$. Distribution ahead of the detonation front (a) and behind the detonation front (b) for $D_{\text {in }}=90 \mathrm{~mm}$ and distribution ahead of the detonation front (c) and behind the detonation front (d) for $D_{i n}=80 \mathrm{~mm}$

The effect of curvature due to the presence of walls in the annular chamber has been addressed. It was concluded that although radial gradients in temperature and pressure are present, they do not affect the overall performance parameters of RDC. The second cause of influence due to the presence of the walls is that of viscous effects. Thus, it is important to evaluate if the prediction of RDC parameters is influenced by the effects of species, momentum and heat diffusion. 
The assessment of the effects of species transport on the numerical simulations of RDC was initially performed in 2D simulations. In the absence of walls, 2D simulation would provide the isolated effect of the species diffusivity. The methodology used to treat diffusive terms and the model selected for the transport properties are described in Section 3.2. Since no appreciable changes were observed in the 2D simulations, the results were not included in this document.

The step following the 2D simulations with the inclusion of diffusive terms consisted of performing 3D simulations where non-slip conditions at the walls were implemented. Despite the realization of the elevated Reynolds number inside the combustion chamber, calculation were initially computed under the assumption of laminar flow. The results obtained do not only provide an initial insight of the effects of non-slip boundary conditions, and the related friction losses, but also serve as a comparison or control point to evaluate the effect of the implementation of a turbulence model in RDC simulations. Figure 4.31 illustrates the temperature distribution in the mid annular plane for a 3D simulation using the PBS and single step reaction mechanism. In addition to the temperature contour, an iso-surface is added to depict the reaction front of the detonation and deflagration region. Although no significant difference is observed in the temperature field with respect to the inviscid solution, oscillations in the deflagration front were observed. It is believed that the velocity gradient caused by the non-slip conditions at the wall leads to the corrugation of the combustion front in the radial direction of the chamber. Due to the lower axial velocity of the injected reactants near the wall, the deflagration front locates closer to the injection plane. On the other hand, the higher axial velocity located near the center of the annular chamber leads to a location of the deflagration front further downstream of the injection plane. The corrugation is presented in the radial direction of the annular chamber and it creates a deflagration front curved inwards towards the injected fresh mixture. In addition to the radial curvature if the deflagration front, oscillations in the azimuthal direction were obtained. Figure 4.31 exemplifies how the white surface representing the fresh mixture boundary does not appear as a planar surface. It is not only curved in the the radial direction, but wrinkled in the azimuthal direction. The presence of wrinkles in the combustion fronts leads to a non-constant fresh mixture column encountered by the detonation wave.

Apart from the corrugation of the deflagration front, no other significant difference in the flow structure of RDC was observed. Moreover, the calculated detonation wave velocity was equal to $1840 \frac{\mathrm{m}}{\mathrm{s}}$, and does not differ greatly from the previously predicted values. Still, changes in the stagnation pressure and temperature at the outlet of the combustion chamber were observed. The stagnation pressure decreased from $2.63 \mathrm{~atm}$ in the inviscid result to $2.58 \mathrm{~atm}$ in the laminar simulation. Even though this is a small change, the $2 \%$ of losses in the stagnation pressure can be attributed to the friction at the wall. On the other hand, the stagnation temperature increased from $1883 \mathrm{~K}$ to $1903 \mathrm{~K}(1 \%)$. It is believed that the increase in stagnation temperature at the outlet may have been caused by the viscous heating generated near the wall due to the high velocity of the detonation products. 


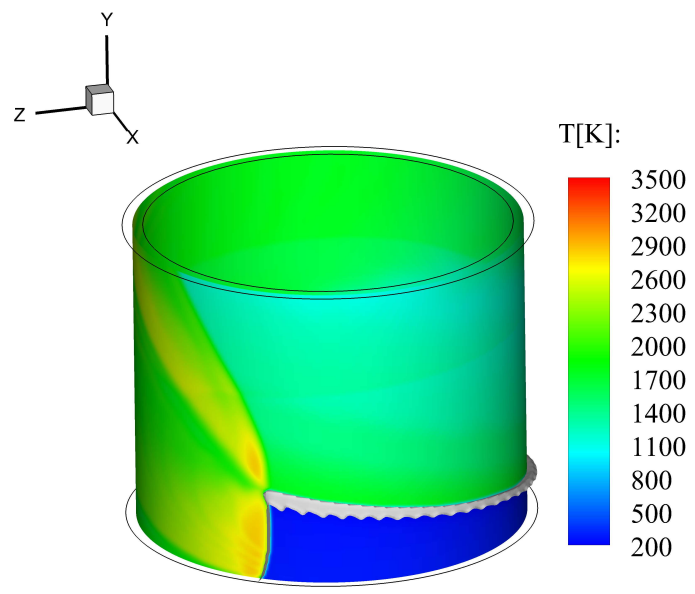

FIGURE 4.31: 3D temperature contour and reaction front surface for 3D laminar and adiabatic simulation

The following step to evaluate the influence of viscous terms on the numerical simulation of RDC was to model the effects of turbulence on the mean flow. As mentioned in Section 3.2, the two equations $\kappa-\epsilon$ turbulence model was selected, for which the wall effects were modeled using the standard wall function formulation developed by [Launder and Spalding, 1974]. For the set of simulations where turbulence is modeled, the wall boundary conditions were treated as non slip and non adiabatic. In such cases, the effects of natural convection and radiation from the external surface of the outer cylinder to the environment were considered. The state for the surroundings of the combustion chamber were taken as air at standard conditions. Additionally, the emissivity for the outer surface was set as 0.85 ; while the heat transfer coefficient was calculated based on the correlations for laminar convection on a horizontal cylinder. The value used for the natural convection coefficient was considered constant throughout the outer surface and not changing in time, . It was calculated based on the correalation for natural convection of a horizontal cylinder, and it was set equal to $8.28 \frac{\mathrm{W}}{\mathrm{m}^{2} \mathrm{~K}}$. The information of the outer cylinder dimensions and materials for the experimental set up in [Liu et al., 2012] were not provided; instead, the conditions implemented in the present study were taken form the experimental combustor described in [Bykovskii and Vedernikov, 2009]. The selection of the study by Bykovskii was based on the detailed information about the external wall cylinder and the similitude with the experimental set up been used as validation in the current study. More importantly, it was selected because of the availability of experimental measurements of heat flux from the combustion chamber to the environment. These measurements are used to validate the predicted heat transfer from the CFD simulations. As mentioned in Section 3.4, the outer wall of the annular combustion chamber was modeled using two different strategies. One strategy assumes that the wall can be treated as a "thin wall" region, while the other incorporates 
the solid region into the computational domain. The results for both strategies are presented as follows:

The first set of results presented correspond to those achieved using the thin wall model. For such boundary condition, the solid region corresponding to the outer wall of the combustion chamber does not need to be meshed, reducing the computational cost. As for the calculation of the heat transfer thorough the wall, ANSYS ${ }^{\circledR}$ Academic Research, Release 14.5. solves the 1D steady state heat conduction equation [Fluent, 2009]. The results obtained using the thin wall boundary condition led to continuous detonation combustion. As shown in Figure 4.32(a), no significant variation in the annular mid plane distribution of temperature was observed. However, by assessing the heat flux distribution at the outer surface of the computational domain (see Figure 4.32(b)), general conclusions on the behavior of heat transfer in RDC can be reached.

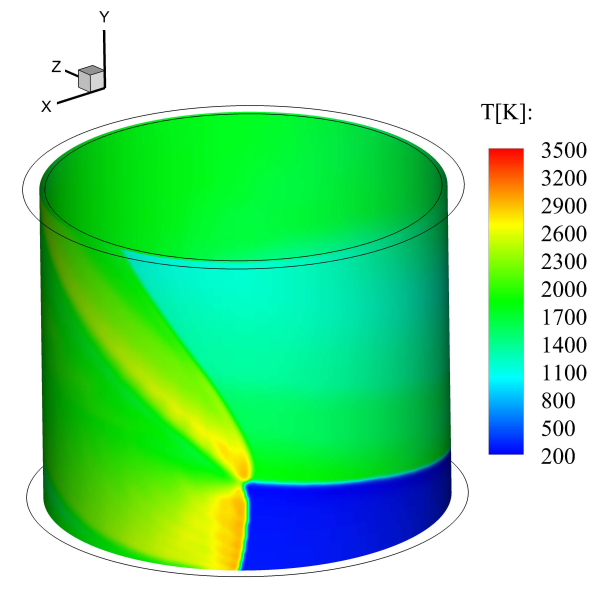

(a)

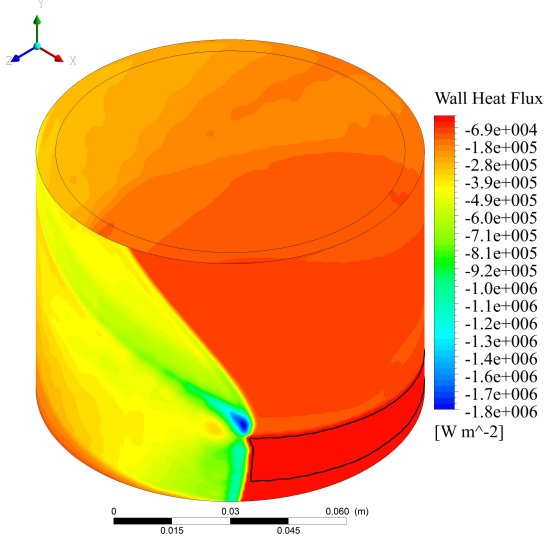

(b)

FIGURE 4.32: Temperature (a) and heat flux (b) contours for thin wall simulation of turbulent RDC

From the distribution heat flux through the outer wall provided in Figure 4.32(b), it is observed that the region where the largest heat flux is present corresponds to the detonation front region; and that the heat flux decreases as the gases expand behind the detonation wave. The region enclosed by the solid line in Figure 4.32(b) represents the portion of the wall where heat is transferred from the outer wall into the fluid. The resulting effect is caused due to the low temperature of the expanding gases in the fresh mixture region. As such, it is observed that a cooling effect of the unreacted mixture in this portion of the wall is captured. That behavior was reported by [Bykovskii and Vedernikov, 2009] in their experimental study. Similar to the treatment given to the pressure data, temperature monitors were distributed in the combustion chamber. For the case of temperature monitors the numerical probes were placed in the computational cell adjacent to the outer wall boundary. The temperature and local heat flux were sampled at various axial locations, but only the data taken at $2 \mathrm{~mm}$ from the inlet plane is illustrated in Figure 4.33. The local heat flux was normalized by the enthalpy influx in the system, 
as given in Equation 4.4. The temperature peak in Figure 4.33(a) corresponds to the passage of the detonation wave, and the comparison with the normalized heat flux plot shows that it is at this instance when the local maximum heat flux occurs. The temperature then decreases as the gases expand, and it drops below the ambient temperature (300K) leading to a small influx of heat from the environment to the domain.

$$
\hat{\dot{q}}_{\text {wall }}=100 \% \frac{\dot{q}_{\text {wall }}^{\prime}}{\dot{m}_{\text {in }}^{h_{\text {react }}}}
$$

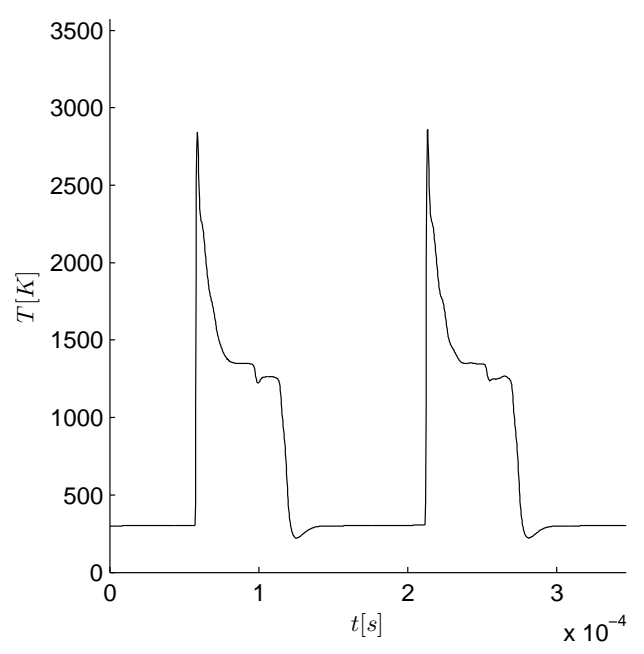

(a)

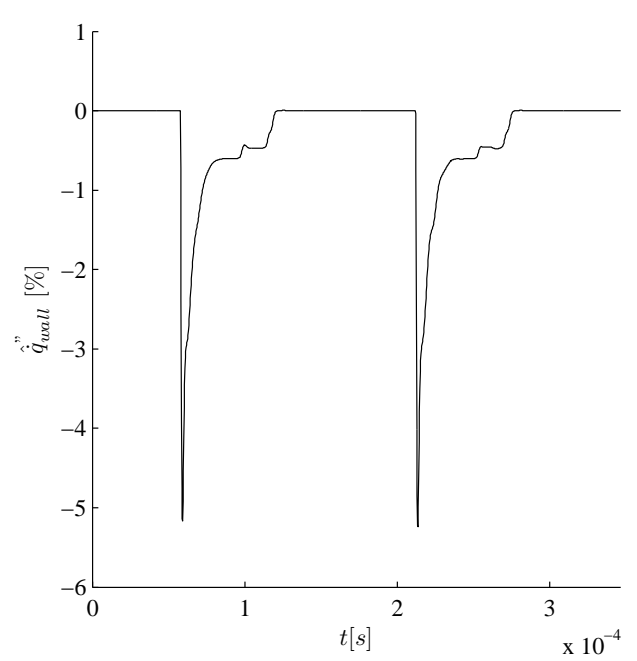

(b)

FIGURE 4.33: Local temperature (a) and normalized heat flux (b) contours for thin wall simulation of turbulent RDC

In additiona to the local sampling of the temperature and heat flux, the integral value of heat transfer through the wall was saved as a function of time. It was obtained that the integral average value of the heat flux through the wall is equal to $0.34 \%$ of the input energy flux. Therefore, it is possible to conclude that the behavior and trend of the temperature and heat flux corresponds to the physical nature of the phenomena. Additionally it is noted that the integral value of the normalized heat flux is in the same order of magnitude than the reported experimental data of $0.5 \%$ given in [Bykovskii and Vedernikov, 2009].

Despite acceptable agreement with experimental data, the thin wall boundary condition does not predict the transient nature of the heat transfer through the wall. Additionally, since the thermal diffusivity of the selected steel is equal to $4.07 e-7 \mathrm{~m}^{2} / \mathrm{s}$, the characteristic time step of conduction through the wall $\left(\tau_{k, \text { wall }}\right)$ - as given in Equation 4.5- is much larger $(250 \mathrm{~s})$ than the period of the detonation wave $\left(1.7 \times 10^{-4} \mathrm{~s}\right)$.

$$
\tau_{k, \text { wall }}=\frac{\Delta_{c}}{\alpha_{\text {wall }}}
$$


In order to study the effects of the unsteady behavior of the heat transferred from the combustion chamber to the outer wall and the environment, a 3D domain including the solid region of the outer wall was created. The initial temperature of the solid region was set equal to the environment temperature $(300 \mathrm{~K})$, and the thermal properties for the solid region were used according to those of steel. The temperature prediction corresponds to the regime of continuous detonation and the heat flux distribution follows the description given for the thin wall boundary condition. It was observed that for the final time of the performed simulation $0.012 \mathrm{~s}$ the temperature in the solid region has increased only by $5^{\circ} \mathrm{K}$.

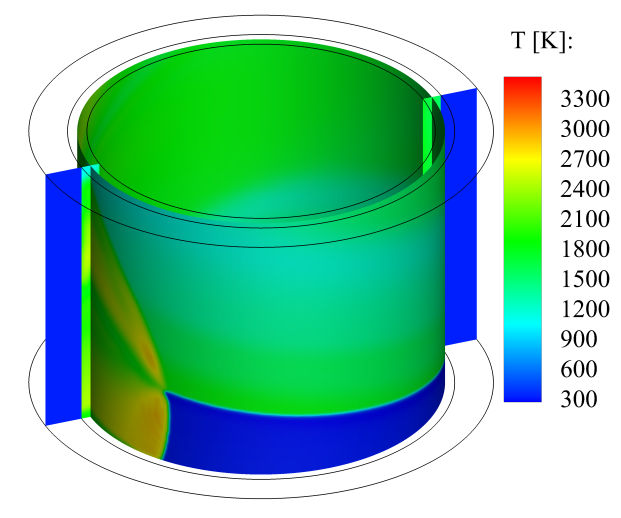

(a)

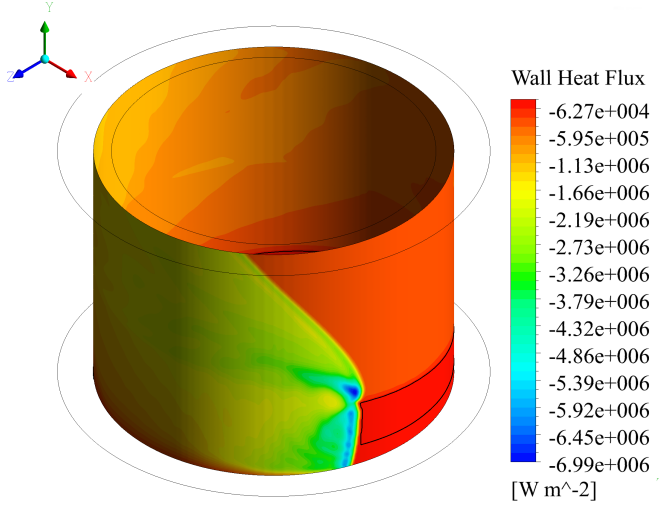

(b)

FIGURE 4.34: Temperature (a) and heat flux (b) contours for 3D simulation of RDC including outer wall solid region

In order to perform a quantitative judgment of the predicted temperature and heat flux, the temporal variation of local heat flux and temperature are illustrated in Figure 4.35. It is seen that the local temperature significantly decreases for the numerical prediction where the solid wall is considered, as when compared to the ones performed using the thin wall boundary condition. This can be explained due to the transient effect considered in the former, and the low initial temperature of the solid region. From the non-dimensional local heat flux $\left(\hat{\dot{q}}_{\text {wall }}\right.$, it is observed that the local maximum heat transfer rate from the detonation products into the solid wall increases by threefold in relation to the solution using the thin wall boundary condition. Additionally the averaged of the integral non dimensional heat flux on the inner surface of the solid region is equal to $1.87 \%$, meaning that it is over predicted with respect to the experimental value. The initial temperature of $300 \mathrm{~K}$ is lower than the temperature that the wall will adopt at steady state or after operation period longer than $0.012 \mathrm{~s}$, the duration of the simulations. The over predicted value can be explained due to the low initial temperature of the solid region.

Summarizing, effects of viscous heat and momentum transfer were considered for 3D RDC simulations. Results were obtained under the following conditions: laminar adiabatic 


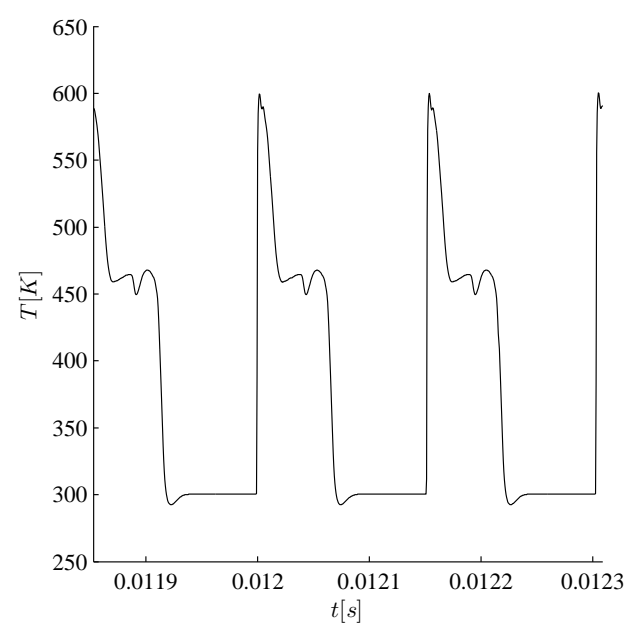

(a)

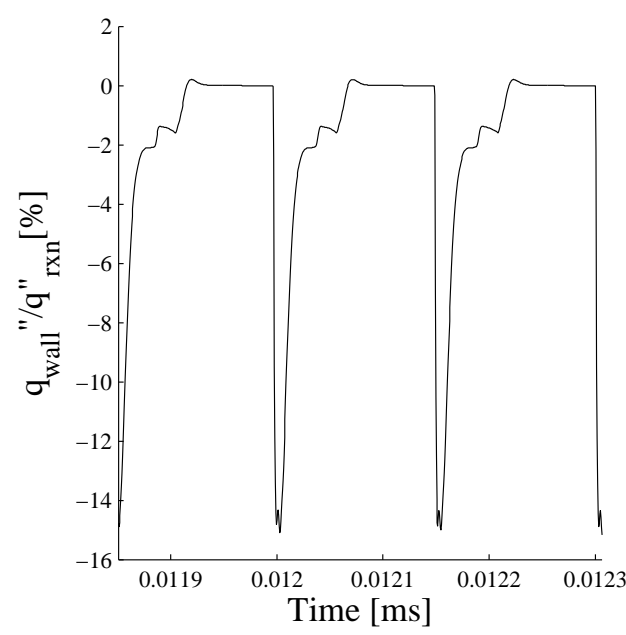

(b)

FIGURE 4.35: Local temperature (a) and normalized heat flux (b) contours for thin wall simulation of turbulent RDC

conditions, turbulent thin wall non-adiabatic, and turbulent non-adiabatic while including the solid region of the outer wall. The results were used to calculate the effects on RDC performance due to friction and heat losses. It was estimated that the total pressure at the outlet $\left(P_{o, \text { outlet }}\right)$ decreased by $1.9 \%, 5.4 \%$ and $4.9 \%$ for the cases of laminar, and turbulent under the thin wall and solid boundary condition, respectively. Additionally, the change in total temperature due to friction effects were quantified. The stagnation temperature at the outlet $\left(T_{o, \text { outlet }}\right)$ increased by $1 \%$ when laminar adiabatic conditions were assumed, it is believed that this was caused due to the effects of viscous heating. However, once the non-adiabatic condition was implemented the $T_{o, \text { outlet }}$ decreased by $0.32 \%$ and $1.38 \%$ for the thin wall and solid wall simulations, respectively. This data will be used to estimate additional losses once the performance of RDC at elevated far field pressures are predicted. A summary of the predicted parameters for the 3D simulations under the different model considerations are given in Table 4.7.

TABLE 4.7: Influence of viscous terms on detonation wave velocity prediction for 3D simulations with PBS-PISO-SQC-2UP and 1 Step reaction mechanism

\begin{tabular}{|c|c|c|c|c|c|c|}
\hline Viscous Model & $\begin{array}{l}\delta x \\
\mathrm{~m}\end{array}$ & $\begin{array}{c}U_{D W} \\
\mathrm{~m} / \mathrm{s}\end{array}$ & $\begin{array}{c}E_{U} \\
\%\end{array}$ & $\begin{array}{c}P_{o, \text { outlet }} \\
\text { atm }\end{array}$ & $\begin{array}{c}T_{o, \text { outlet }} \\
\mathrm{K}\end{array}$ & $\begin{array}{c}\hat{\dot{q}}_{\text {wall }}[\%] \\
\%\end{array}$ \\
\hline Inviscid & 0.5 & 1848 & 6.0 & 2.63 & 1885 & 0.0 \\
\hline Laminar-Adiabatic & 0.25 & 1840 & 5.6 & 2.58 & 1903 & 0.0 \\
\hline Turbulent-Non-Adiabatic Thin Wall & 0.25 & $1932 *$ & 10.84 & 2.47 & 1879 & 0.35 \\
\hline Turbulent-Non-Adiabatic Solid Wall & 0.25 & $1966 *$ & 12.8 & 2.50 & 1859 & 1.89 \\
\hline
\end{tabular}

Separately from the influence of viscous effects, additional 3D RDC simulations incorporating the injection geometry were also performed. This set of simulations was used to study 
the influence of calculating the conditions instead of being incorporated in the computational domain. The numerical predictions where the injection section is included in the computational domain were performed using the 3D mesh illustrated in Figure 3.2(a). The inlet boundary condition was specified as decribed in Section 3.4. In this set of simulations, viscous effects were not considered and only the 1 step reaction mechanism was used. It was also assumed that the fuel and oxidizer enter the domain in a perfectly premixed state. In order to avoid premature initiation of the reactions, it was assumed that the combustion can only occur downstream of the expansion plane. Hence chemical reactions were disabled in the injection manifold and convergence-divergence portion of the injection nozzle. In order to obtain continuous detonation combustion, the unreacted solution of the flow inside the combustion chamber was first obtained. The unidirectional initiation of the detonation wave was performed by interpolating the analytical solution from the adapted 1D model into the 3D domain (see Section 3.5). Continuous detonation combustion was obtained following the previously described process. As illustrated by the temperature contour at the annular mid plane in Figure 4.36, 3D influences on the flow structures were observed. The deflagration region is not a smooth uniform planar surface as predicted in the $2 \mathrm{D}$ and $3 \mathrm{D}$ simulations where the ideal injection model was implemented. Instead, it presents great levels of corrugation; where the deflagration front advances further downstream for the region near the outer wall (see Figure 4.36). Similar to what was obtained for the 3D simulations with $D_{i n}=95 \mathrm{~mm}$, pockets of unreacted or partially reacted mixture are formed within the detonation products.

The irregular deflagration front, in this set of simulations, is caused by the non uniform distribution of injection velocity at the injection plane. Such uneven distribution is evident in Figure 4.37. The results show that the injection velocity is negative at the location of the detonation front, and that it increases more rapidly near the outer wall behind the detonation front. However, this trend changes as the azimuthal distance from the reaction front increases. The variation on injection velocity in the radial and azimuthal direction leads to the previously described shape of the deflagration front.

The pressure variation with time was sampled using numerical probes in different axial location of the combustion chamber and injection section. Figure 4.38 provides the pressure history at the points located in the inlet surface, the throat of the injection nozzle and at the expansion plane. It is observed that there is a small upstream influence of the detonation wave in the injection surface and at the throat of the injection nozzle. The upstream influence of the detonation wave occurs because a portion of the injection section do not operate under choked conditions. In other words, the high pressures at the detonation front and immediately behind of it lead to a high back pressure for the injection section. Under such high pressure, the injection section does not operate at chocked conditions and upstream influence can occur. 


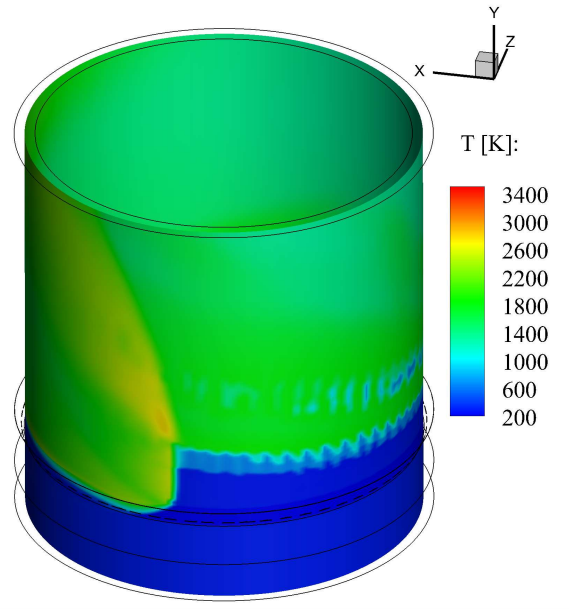

(a)

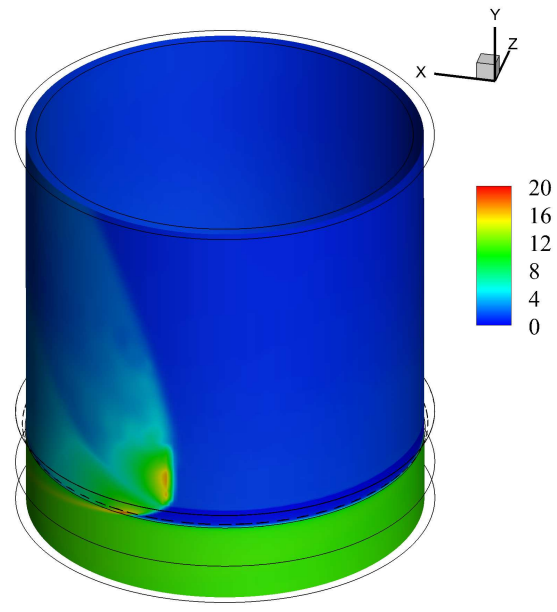

(b)

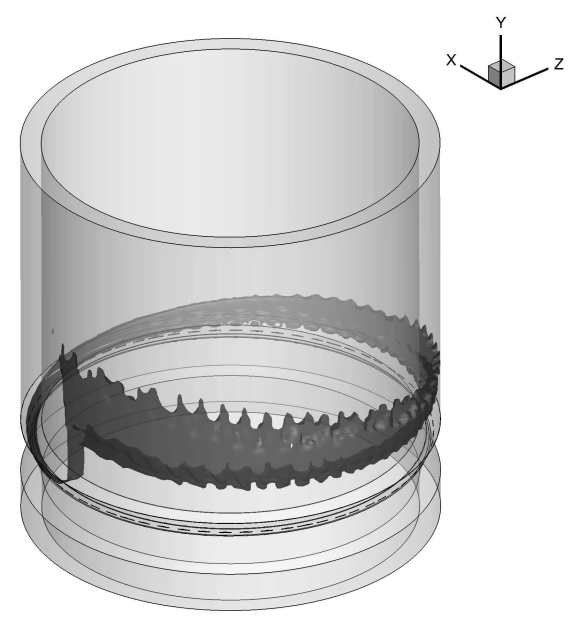

(c)

FiguRE 4.36: Temperature (a), pressure (b) and reaction front (c) contour plots for 3D RDC with injection section

It was also observed that the pressure history at the expansion planes correlates appropriately with those predicted while using the injection model. The lower pressure peak are attributed due to the low mesh resolution used in during this simulations $(\delta x=0.5 \mathrm{~mm})$. Despite the larger computational cells, the predicted detonation wave velocity is equal to $1936 \frac{\mathrm{m}}{\mathrm{s}}$, an $11 \%$ difference with respect to the experimental detonation wave velocity. Additionally, the predicted stagnation pressure and temperature at the outlet were equal to $2.54 \mathrm{~atm}$ and $1892 \mathrm{~K}$, respectively.

It is then concluded that $3 \mathrm{D}$ simulations performed considering the injection section evidence modes of instabilities in the deflagration front that are not captured if the ideal injection model is used. It was obtained that the total pressure decreased by approximately $3.4 \%$. It is then important to consider these observations for the simulation of RDC at elevated pressure. If 


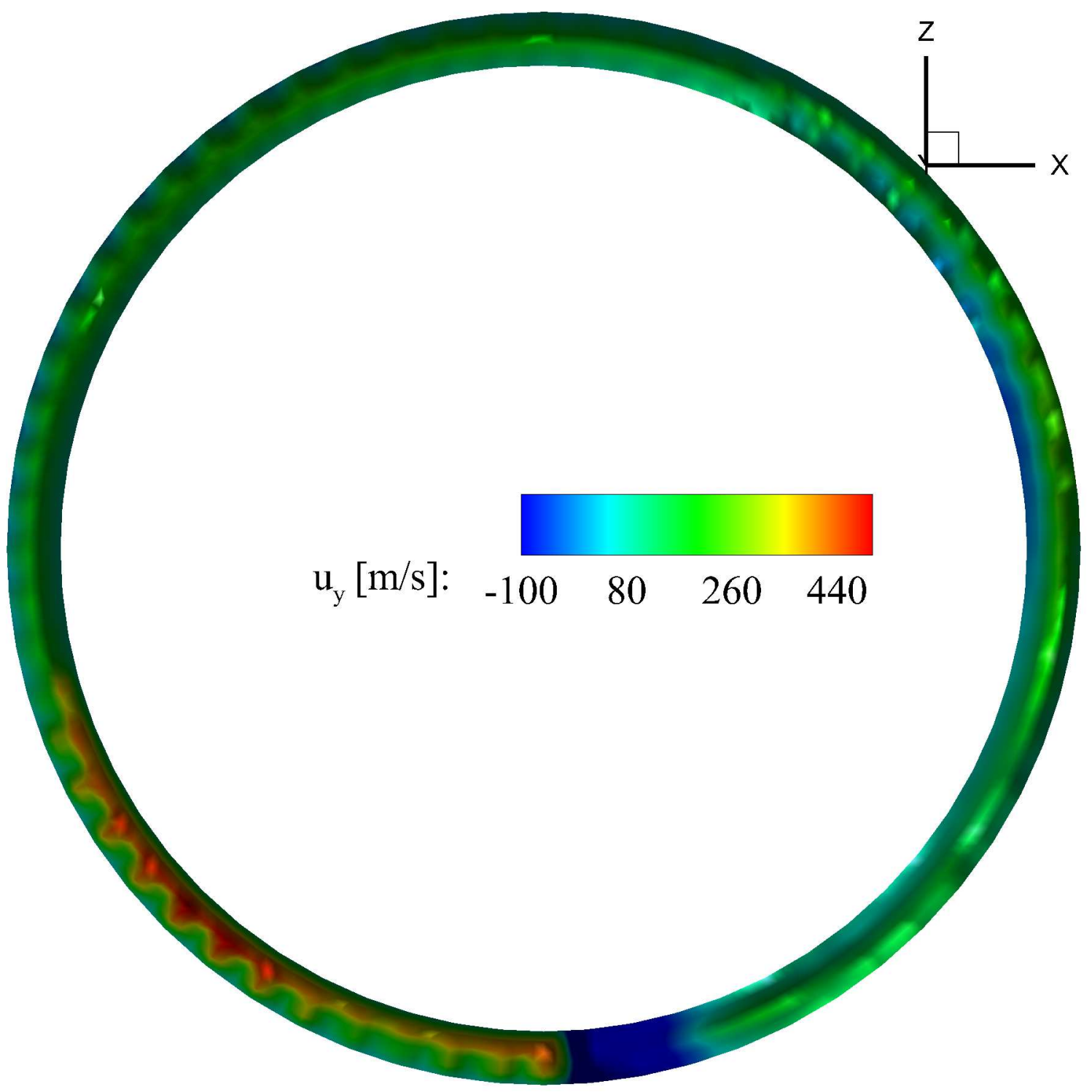

(a)

FIGURE 4.37: Cross sectional axial velocity contour from 3D simulation for RDC chamber with injection geometry

the far field pressure is close to the limiting pressure for continuous detonation, the presence of the irregularities in the combustion front may restrict the occurrence of continuous detonation.

\subsection{Conclusions from Model Validation}

- Two types of CFD solvers and multiple discretizations schemes were evaluated and validated for the prediction of the non reactive shock tube problem. It was concluded that under the different numerical configurations evaluated, the distribution of pressure and the 


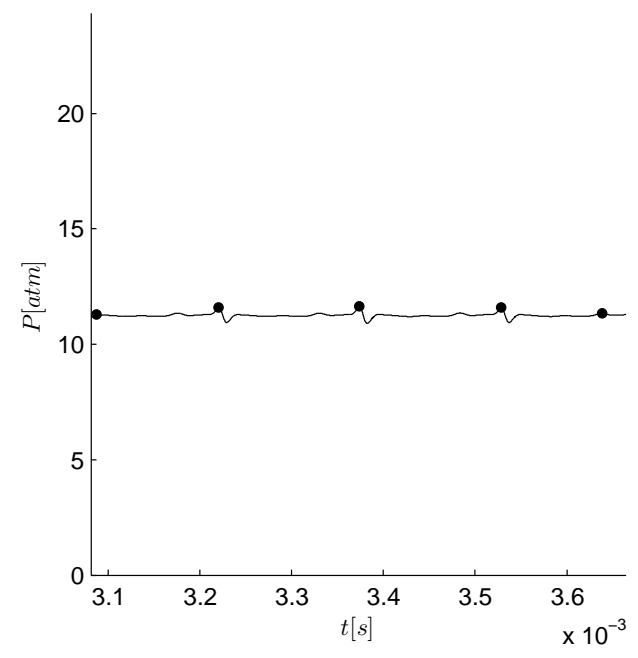

(a)

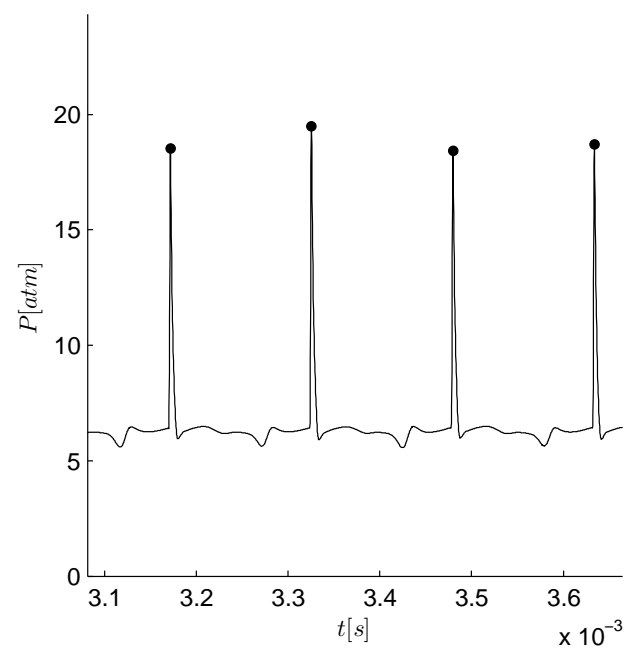

(b)

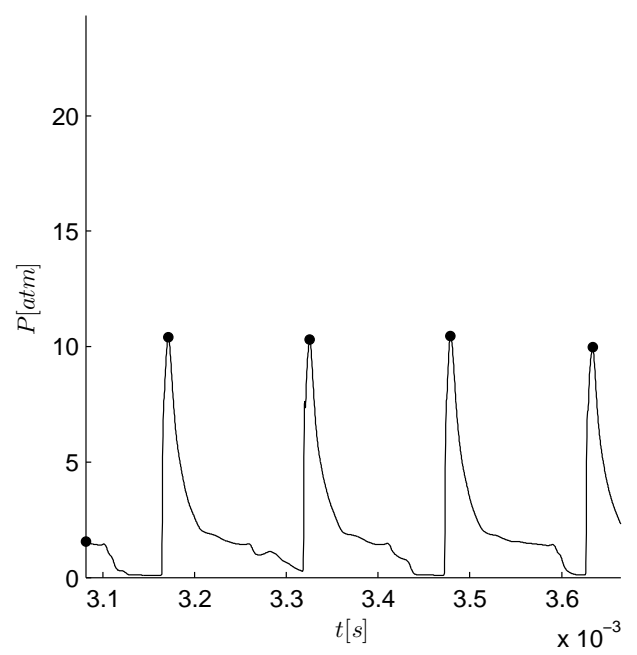

(c)

FIGURE 4.38: Pressure history for 3D RDC including injection section. Data sampled at inlet (a), throat (b) and expansion (c) plane

velocity of the shock wave are adequately predicted. Error as low as $0.3 \%$ were obtained with computational cell sizes of $1 \mathrm{~mm}$.

- The error analysis performed on the numerical prediction for the non reactive shock tube problem led to the conclusion that the solution strategies implemented have a convergence order that between first and second order, while using a first order time discretization scheme. When second order temporal discretization was implemented numerical "wiggles" in the pressure predictions were evident, and the convergence order was not increased.

- The effects of the selected chemical models in the prediction of the CJ state and ZND detonation wave structure were evaluated. Differences in the detonation wave structure and dominant length scales were covered. It was concluded that despite the differences in the 
induction region and heat release zone between the 1 step and multi step chemical model, both mechanisms adequately predict the overall parameters of the detonation wave. The multi step model is recognized as the strategy that better predicts the characteristic ZND structure; however, the 1 step reaction mechanism overcomes such drawback due to its low computational cost and versatility to be adapted for different reactive mixtures. This conclusion is also supported by the fact that the present study does not focus on predicting the detailed structure of the detonation front, but on analyzing the general performance parameters in RDC.

- CFD simulations for the propagation of a planar detonation wave in a $2 \mathrm{D}$ channel were performed in order to evaluate the performance of the selected numerical strategy and chemical models. It was concluded that the detonation wave velocity and the general behavior of the detonation wave structures are appropriately predicted. Relative errors calculated with respect to the CJ conditions show that for $\delta x=0.1 \mathrm{~mm}$ the predicted values do not differ with the theoretical solution for more than $1 \%$. Such results led to the selection of that specific mesh resolution for the 2D simulation of RDC presented in the following sections.

- An experimental case reported in the literature was selected for model validation of the $2 \mathrm{D}$ and 3D simulations. The selected geometry and operating conditions lead to supersonic exhaust of the detonation products.

- Two dimensional simulations of RDC for the validation conditions were preformed. It was concluded that predictions using the 1 step and multi step chemical model led to the adequate prediction of the flow structures in RDC. Additionally, by comparing the experimental and computational pressure history it was concluded that appropriate prediction of the pressure field in RDC was obtained. Moreover, the predicted detonation wave velocity showed a relative error of $7 \%$, when compared to the experimental data.

- The 3D effects on laminar RDC simulations were addressed. The presence of radial variations in temperature and pressure were evidenced for different values of $\left(\Delta_{c} / D_{\text {mean }}\right)$. It was concluded that despite the presence of such variations the overall performance parameters of RDC due not vary significantly.

- The prediction of major species and pollutant concentration was obtain for RDC under the operating conditions of the validation case. For stoichiometric $\mathrm{H}_{2}$-air operations, a time averaged concentration of dried $\mathrm{NO}$ corrected for $15 \% \mathrm{O}_{2}$ at the combustion chamber equal to $164 \mathrm{ppm}$ was predicted. Supplemental simulations were performed for an injection mixture composition with $\Phi=0.5$. Under such conditions, the predicted concentration of dried NO corrected for $15 \% \mathrm{O}_{2}$ was equal to $35 \mathrm{ppm}$, a reduction of $79 \%$. 
- The effects of laminar and turbulent diffusive terms were evaluated for 3D simulations of RDC. The predicted values of the total pressure losses due to the turbulent friction terms was equal to $0.13 \mathrm{~atm}$.

- The effects of heat transfer at the wall in 3D simulations of RDC were analyzed. It was obtained the $0.3 \%$ and $1.3 \%$ of the influx of energy is dissipated through the wall, depending of the wall boundary condition implemented. The predicted heat flux values are within the experimental measurements reported by [Bykovskii and Vedernikov, 2009]. 


\section{Chapter 5}

\section{Rotating Detonation Combustion at Elevated Pressures}

The present chapter addresses the issues of numerical simulations of RDC at elevated outlet pressures. First, the analytical model developed for the prediction of geometrical and operating conditions favorable for continuous detonation and pressure gain combustion in RDC is presented. Second, the 2D CFD simulations for RDC at elevated far field pressures are covered. In this section, further details on the treatment and formulation of the partially reflective boundary conditions are provided, and conclusions on the favorable conditions for PGC in RDC are described.

\subsection{Adaptation of 1D Model as a Predictive Tool}

An analytical model to predict the pressure, temperature and species distributions in annular combustion chamber for RDC operations was developed. The developed model consists of the integration of the 1D RDC model developed by [Nicholls and Cullen, 1964] and the injection model presented by [Zhdan et al., 1990]. The integration was performed in order to predict the axial variation of temperature and species in RDC; and to estimate the limiting pressure for which continuous detonation can be achieved. The model was also used to obtain a first approximation on the possibility of achieving PGC in RDC at elevated outlet pressures. The developed analytical model, despite of been based on the integration between two existent analytical submodels, represents an important contribution towards the preliminary design process of rotating detonation combustion devices.It not only permits to quickly estimate the operational range at which continuous detonation can be achieved, but also produces an initial condition for the simulation of RDC. Due to the proximity of the analytical solution to the numerical CFD solution at the periodic steady state, the computational time dedicated to eliminate the transient behavior 
due to the initial condition is reduced. It was also proven that the developed analytical model and its resulting initial conditions, enabled the numerical initiation of RDC at elevated outlet pressures.

As described in Section 2.4, the 1D RDC model developed by [Nicholls and Cullen, 1964] is based on the assumption that the variation of the thermodynamic state of the detonation products in the axial direction of the combustion chamber can be neglected. The inputs required in the model by [Nicholls and Cullen, 1964] are the thermodynamic state and chemical composition of the gases ahead of the detonation wave. The solution of the 1D model equations leads to the distribution of pressure, temperature and Mach number in the azimuthal direction of the RDC annular chamber.

In the model developed as part of the current research effort, a 2D distribution of temperature and species is obtained by predicting the location of the deflagration front in the combustion chamber. The predicted location of the deflagration front is used to determine the regions where reactants or detonation products exist inside the annular chamber. The manner in which this predictions are achieved as follows: First, the 1D distribution of temperature, pressure and Mach number in the azimuthal direction is calculated using the [Nicholls and Cullen, 1964] model. For such calculations, it is assumed that the pressure ahead of the detonation wave is equal to $P_{\infty}$, with the exception of cases where the far field pressure is lower than the atmospheric pressure. Under such conditions, the pressure ahead of the detonation wave is assumed to be equal to 1.0atm. The predicted distribution of pressure behind the detonation wave is then used as input for the injection model. The supplemental required parameters are the characteristics and operating conditions of the injection section, and the propagation velocity of the deflagration front. The injection model, as described by Equation 3.13 to Equation 3.18, is then used to predict the local thermodynamic state of the reactants at the injection plane. The predicted injection velocity $\left(\left.\vec{v}(\vec{x})\right|_{\text {inlet }}\right)$ is used to calculate the distance reached by the reactants in one cycle of the detonation wave. Both the the injection velocity and the predicted distance are a function of the azimuthal location. The local distance predicted is corrected using the propagation velocity of the deflagration front. As a result, a distribution in the azimuthal direction of the distance reached by the injected reactant in one detonation wave cycle is obtained. This distance is then assumed as the location of the deflagration front. Moreover, the maximum value of the predicted location is taken as the detonation front height $\left(\Delta_{D W}\right)$. Additionally, the high pressure region behind of the detonation front leads to zero injection velocities in certain areas of the inlet (see Section 3.4). Therefore, the geometric parameter $\left(\theta_{R}\right)$ is defined as the portion of the inlet that experiences injection velocities greater than zero. Once the geometry of the detonation front has been established, it is assumed that the pressure and temperature above the detonation front height are equal to the predicted value of the detonation products at the furthest point from the detonation wave. The predicted temperature and species distribution calculated using the previously described model were presented in Figure 3.5. 
The solution calculated using the analytical model was compared with the results obtained from 2D CFD simulation (see Figure 5.1). It is observed that the pressure distribution behind the detonation wave is appropriately predicted. Despite the difference in the predicted location of the deflagration front, $\Delta_{D W}$ correlates appropriately with the $2 \mathrm{D}$ CFD results. The prediction of the detonation front height is of great importance for the present research, since it is one of the parameters that determines the possibility of obtaining continuous detonation.

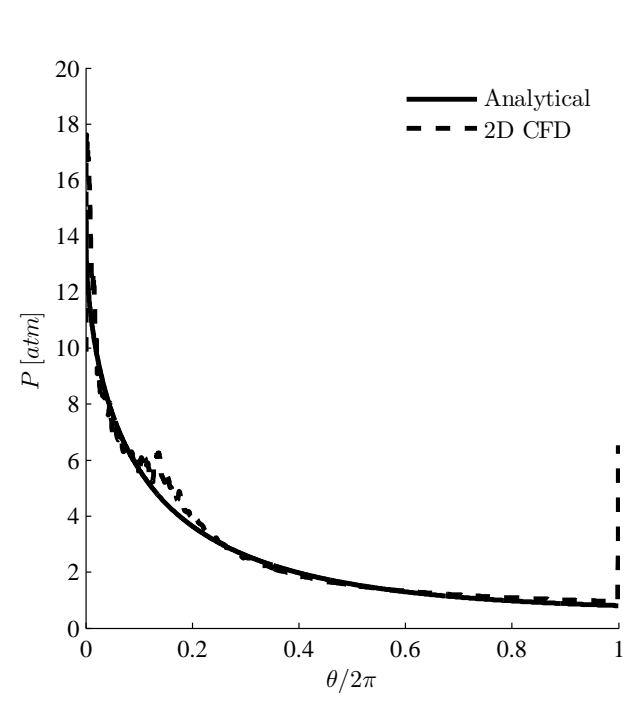

(a)

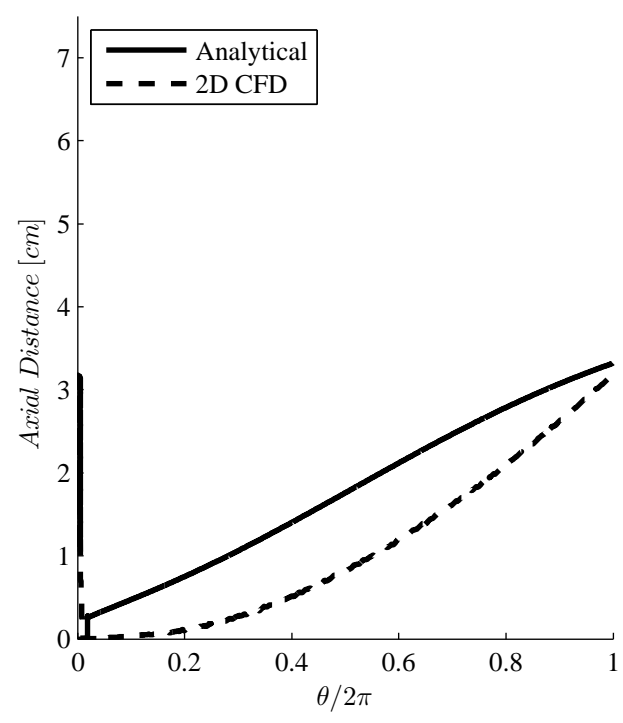

(b)

FIGURE 5.1: Comparison of analytical model prediction with 2D CFD results. Pressure (a), and deflagration front location (b)

After an initial corroboration of the capabilities of the developed analytical tool, it was possible to use such model for exploratory calculations of RDC at off design operating conditions. The calculations were based on the experimental set up by [Liu et al., 2012], and a parametric study varying $A_{t} / A_{e}$ ratio and the value of $P_{\infty}$ was performed. The results show that the predicted distribution of static pressure behind the detonation wave is not influenced by the injection geometry or its operating conditions. However, as seen in Figure 5.2, it is a strong function of the static pressure ahead of the detonation wave. The predictions from the analytical model show that the pressure increases substantially behind the detonation wave once $P_{\infty}$ increases from $1.0 \mathrm{~atm}$ to $7.0 \mathrm{~atm}$.

Due to the changes in the static pressure distribution behind the detonation wave, alteration of the deflagration front shape was observed. The predicted deflagration front geometries for three different injection area ratios $\left(A_{t} / A_{e}\right)$ and four different values of $P_{\infty}$ are presented in Figure 5.3. In general, the detonation wave height $\left(\Delta_{D W}\right)$ decreases as $P_{\infty}$ increases, while the portion of the injection section through which reactants are injected $\left(\theta_{R}\right)$ decreases when $P_{\infty}$ increases. These two effects are in detriment to the possibility of obtaining continuous detonation. However, it was also observed that by increasing the throat to expansion area ratio $\left(A_{t} / A_{e}\right)$ 


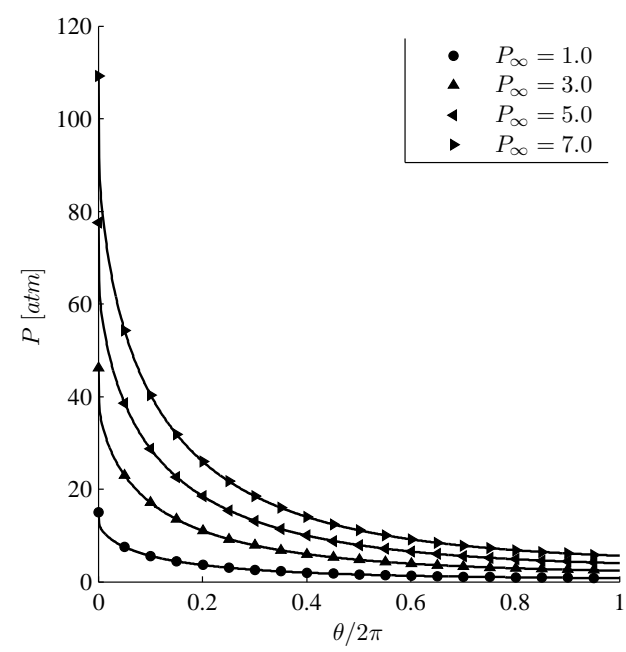

FIGURE 5.2: 2D Analytical model prediction of static pressure for RDC at elevated pressures

the injection velocity is increased, which leads to a larger $\Delta_{D W}$. In fact for $A_{t} / A_{e}=0.04$ and $P_{\infty}=1.0 \mathrm{~atm}, \Delta_{D W}$ exceeds the length of the combustion chamber (see Section 5.3). Operation under such a regime is counter productive, since it would lead to unreacted mixture escaping the combustion chamber.

In order to facilitate the characterization of the deflagration front shape and the detonation wave height, a non dimensional factor $(\Theta)$ was defined. $\Theta$ was defined in order to provide a metric on the possibility of obtaining continuous detonation under certain operating and injection conditions. It was defined as a non dimensional factor that incorporates the area ratio of the inlet at which reactants are injected $\left(\frac{\theta_{R}}{2 \pi}\right)$ and the ratio of the detonation height with respect to the length of the combustion chamber $\left(\frac{\Delta_{D W}}{L_{c}}\right)$. The definition of $\Theta$-given in Equation 5.1- determines that under conditions favorable for continuous detonation, $\Theta$ would take at values closer to 1 . On the other hand for conditions under which continuous detonation can not be achieved, the value of $\Theta$ would decrease. It is believed that for a fixed chamber length, a limiting value of $\Theta$ determines the possibility of obtaining continuous detonation. The predicted values of $\Theta$, for the before mentioned conditions are given in Figure 5.4. The values of $\Theta>1.0$ in Figure 5.4 correspond to the predictions where the detonation front height $\left(\Delta_{D W}\right)$ exceeds the length of the combustion chamber $\left(L_{c}\right)$. From the illustrated variation of $\Theta$ given in Figure 5.4, it can the be concluded that increasing the area ratio $\left(A_{t} / A_{e}\right)$ increases the likelihood of obtaining continuous detonation for a fixed value of $P_{\infty}$. Despite the fact that this trend has been identified, it is necessary to determine the limiting or cutoff value $\Theta_{c}$.

$$
\Theta \equiv\left(\frac{\theta_{R}}{2 \pi}\right)\left(\frac{\Delta_{D W}}{L_{c}}\right)
$$

In addition to the calculation of the geometrical factor $(\Theta)$, the estimated total pressure loss $\left(\% P_{o, l o s s}\right)$ were calculated. The average value of stagnation pressure was calculated based 


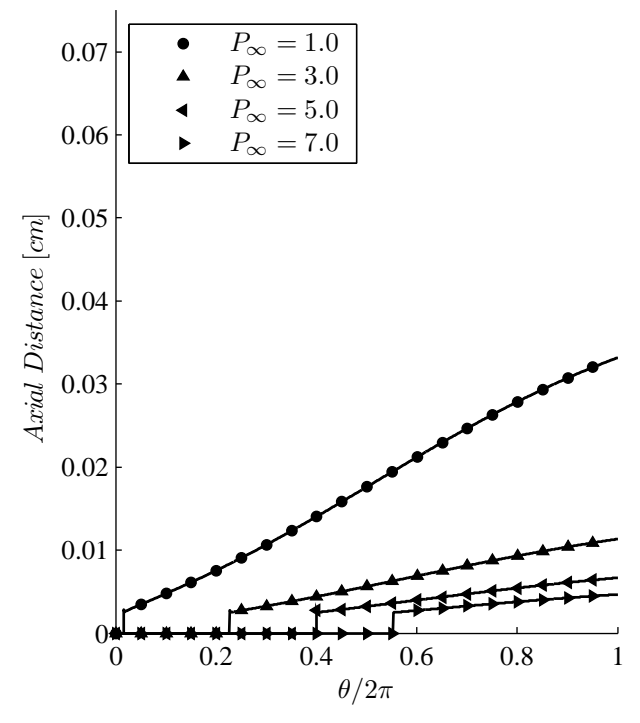

(a)

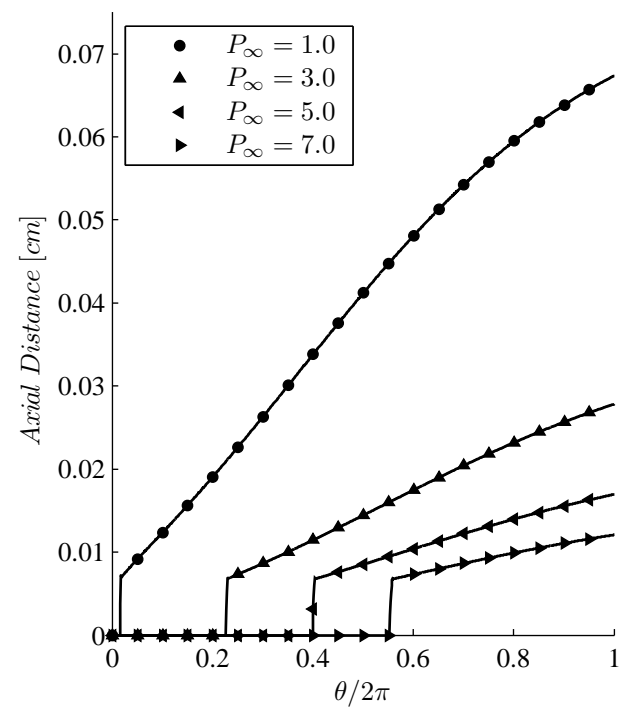

(b)

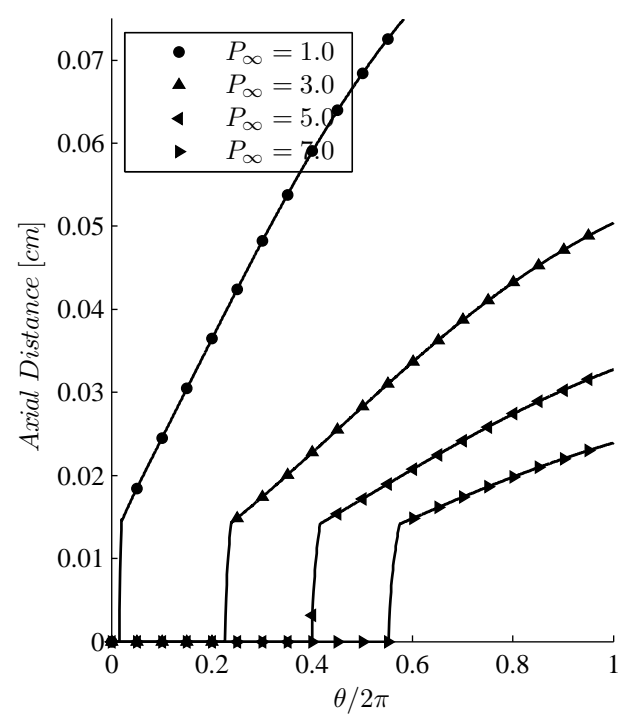

(c)

FIGURE 5.3: Analytical model prediction of deflagration front shape for RDC at elevated pressures and various injection geometries. $A_{t} / A e=0.08(\mathrm{a}), A_{t} / A e=0.2(\mathrm{~b})$ and $A_{t} / A e=0.4(\mathrm{c})$

on the $1 \mathrm{D}$ prediction and the injection velocity of the reactants. The variation of $\% P_{o, l o s s}$ with changes in pressure and injection geometry are illustrated in Figure 5.5. A large pressure gain is predicted for low values of $P_{\infty}$ and $A_{t} / A_{e}=0.4$. This region corresponds to the supersonic injection of the reactants where no losses in total pressure in the injection section are presented. The operation under this regime is not of much interest to the present study, and will be disregarded. The region of relevance for the current study corresponds to that of elevated values of $P_{\infty}$. It is observed that as the far field pressure is increased the possibility of obtaining PGC also increases. Despite the fact that the predicted values are presented for all the geometric configurations, it should be kept in mind that the possibility of continuous detonation decreases as $P_{\infty}$ 


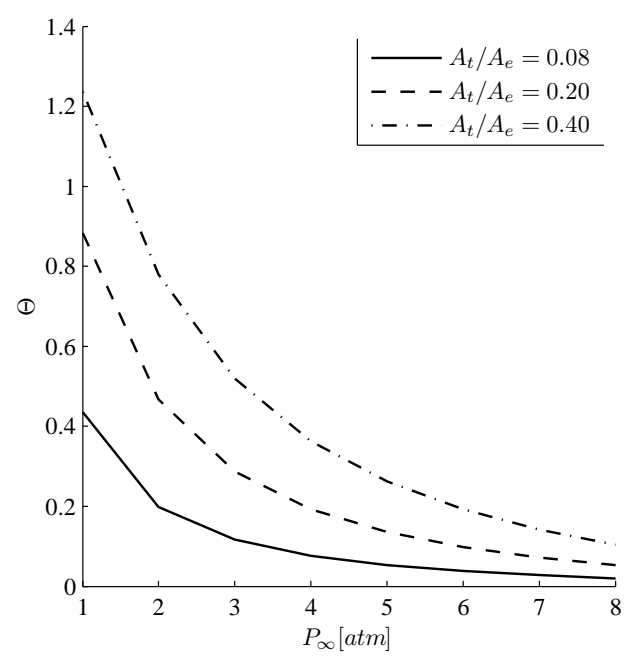

FIGURE 5.4: Analytical model prediction of $\Theta$, influence of $P_{\infty}$ and $A_{t} / A_{e}$

increases, due to the decrease in $\Delta_{D W}$.

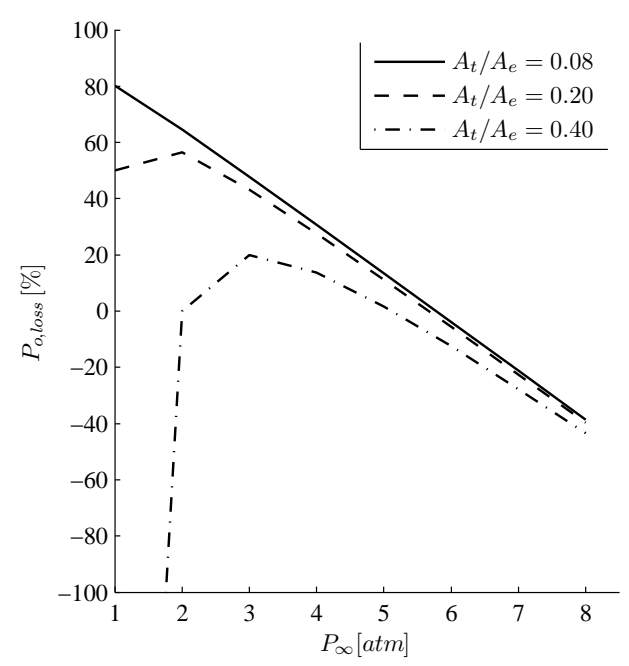

FIGURE 5.5: Analytical model prediction of losses in total pressure in RDC; influence of $P_{\infty}$ and $A_{t} / A_{e}$

Based on the parametric study performed using the developed analytical model, it is possible to initially establish favorable conditions for continuous detonation and PGC in RDC. The results show that for $P_{\infty}$ relevant for GT applications, using low $A_{t} / A_{e}$ ratios it is unlikely to obtain continuous detonation. Instead larger values of $A_{t} / A_{e}$ should be implemented. Additionally it was observed that increasing $P_{\infty}$ favors the occurrence of PGC in RDC. Despite the fact that significant assumptions are made in the formulation of the developed model, its ease of implementation and low computational time makes this model a valuable tool for preliminary calculations. Information from the $2 \mathrm{D}$ simulations is used in order to establish the reliability of the analytical results. 


\subsection{Two Dimensional Simulations of RDC at Elevated Pressures}

In the current section the two dimensional simulations of RDC at elevated $P_{\infty}$ are presented. The limiting conditions for different injection geometries are determined, and the pressure losses are quantified. However, before presenting the results further clarification on the treatment of the outlet pressure boundary condition are provided.

\subsubsection{Application of Non Reflective Boundary Condition for RDC Simulations}

In order to appropriately predict the performance of RDC under elevated far field pressure, the treatment of the outlet boundary must be carefully performed. The current study addresses a strategy to select the appropriate value for the relaxation factor $(\sigma)$ necessary for the adequate implementation of the NRBC.

In order to demonstrate the need for the adequate selection of $\sigma$, results using a value of $\sigma=0.15$ are initially presented. For such simulations, the selected combustion and injection geometry corresponds the experimental set up used for model validation in the current study ([Liu et al., 2012]). The only change performed to the operating conditions was increasing $P_{\infty}$ from the original $0.11 \mathrm{~atm}$ to $2.0 \mathrm{~atm}$. It is expected that $P_{\infty}=2.0 \mathrm{~atm}$ leads to subsonic exhaust of the detonation products. The results obtained using $\sigma=0.15$ showed that the simulation drifts off from the imposed outlet boundary condition. This is made evident by the history of the average pressure at the outlet presented in Figure 5.6. It was observed that despite imposing a far field pressure equal to 2.0atm, the average outlet pressure oscillates around 3.75atm.

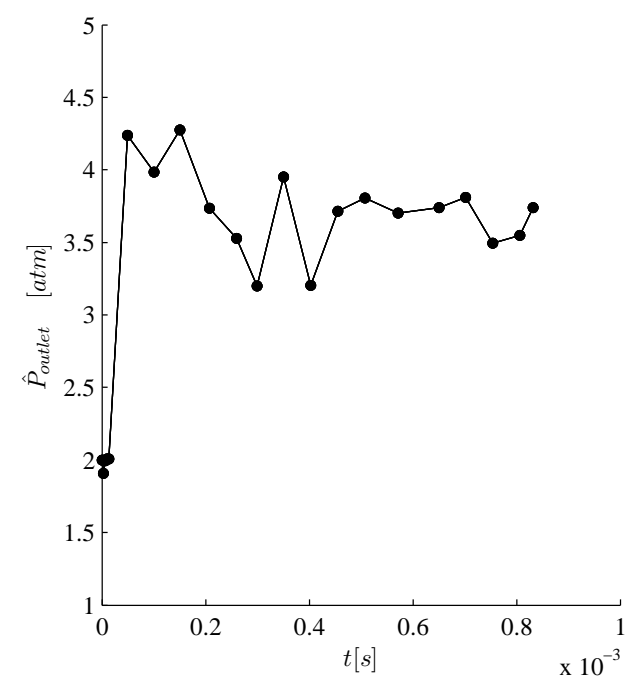

FIGURE 5.6: Outlet pressure prediction with $\sigma=0.15$, ill-imposed problem

Based on the theory by [Selle et al., 2004] for non-reflective boundary condition (NRBC) and the linear relaxation method (LRM), an estimation for the appropriate relaxation factor $(\sigma)$ 
was calculated. In order to establish the adequate value of $\sigma$, the dominant frequencies of the system to be modeled need to be known. For the current study, a power-spectral analysis on the pressure signal from the 2D CFD simulations for Hydrogen-Air RDC at $P_{\infty}=0.11 \mathrm{~atm}$ was performed. It was obtained that the dominant frequencies correspond to $6.3,12.6$ and $18.98 \mathrm{kHz}$. The value of $\sigma$ was then calculated as given in Equation 5.2. The resultant value of $\sigma$ for the current simulations is equal to $-2.1 \pi$. The selected $\sigma$ has a negative sign since the calculation of $K$ (see Equation 3.21) in ANSYS ${ }^{\circledR}$ Academic Research, Release 14.5 is based on $M_{\text {max }}$, which for RDC is always greater than 1 . This is a small but critical issue, if the sign of the relaxation factor $\sigma$ is overlooked the mean flow pressure at the boundary is not controlled.

$$
\sigma=\frac{4 \pi L_{c} f_{\min }}{c\left(1-M_{\max }^{2}\right)}
$$

Based on the selected value of $\sigma$, the theoretical reflection coefficient $\left(R_{f}=\frac{\mathcal{L}_{1}}{\mathcal{L}_{5}}\right)$ can be calculated for the governing frequencies inside the RDC annular chamber. $R_{f}$ is calculated based on the work by [Selle et al., 2004], and as given in Equation 5.3, where $\omega$ are the angular frequencies of the dominant modes inside the combustion chamber. The illustration of the variation of $R_{f}$ with $\sigma$ for the governing frequencies of RDC is given in Figure 5.7. It is observed that for $\sigma=-2 \pi$, the lower frequency pressure waves lead to larger reflection coefficients. This means that pressure waves with lower frequencies are partially reflected at the outlet boundary, while higher frequency waves are not.

$$
\left\|R_{f}\right\|=\frac{1}{\sqrt{1+\frac{2 \omega^{2}}{K}}}
$$

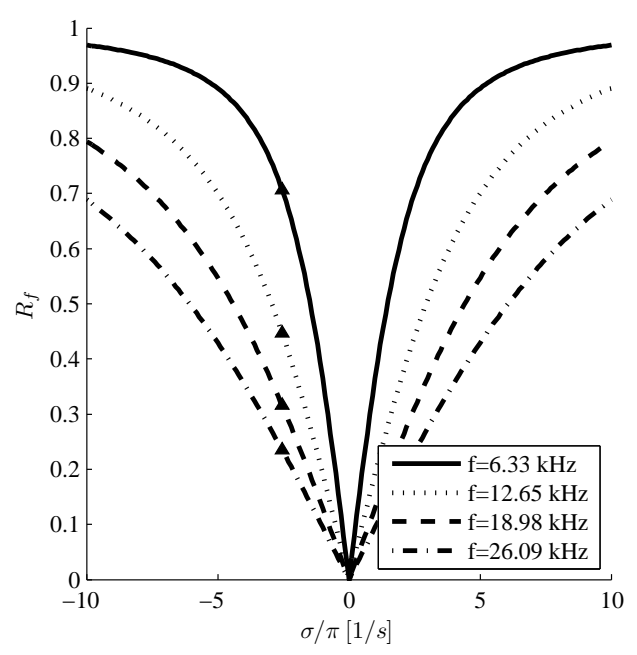

FIGURE 5.7: Theoretical reflection coefficient for the NRBC with $\sigma=-2 \pi$. $\Delta$ represents the selected value of $\sigma / \pi$ 
In order to evaluate the selection of $\sigma$, a 2D CFD simulation of RDC with $P_{\infty}=2.0 \mathrm{~atm}$ was performed. For such study, the averaged static pressure at the outlet boundary $\left(\hat{P}_{\text {outlet }}\right)$ was monitored as a function of time. As illustrated in Figure 5.8, the appropriate selection of $\sigma$ leads to the control of the the pressure in the mean flow. It was also obtained that the average outlet pressure stabilizes after initial fluctuations at a pressure equal to $2.3 \mathrm{~atm}$.

The selection of the ideal value of $\sigma$ was performed based on a cutoff frequency equal to $6.33 \mathrm{kHz}$. Meaning the pressure disturbances with frequencies higher that the cutoff frequencies are less likely to be reflected, while lower frequency disturbances are partially reflected, but they induce control on the mean flow pressure. Simulations performed with $-2.1 \pi<\sigma<0$ (closer to zero), lead to less reflection of pressure disturbances, but present the problem of the outlet pressure drifting from the imposed $P_{\infty}$ value. On the other hand if $\sigma<-2.1 \pi$, the far field pressure is still controlled but the magnitude of the reflection coefficient increases.

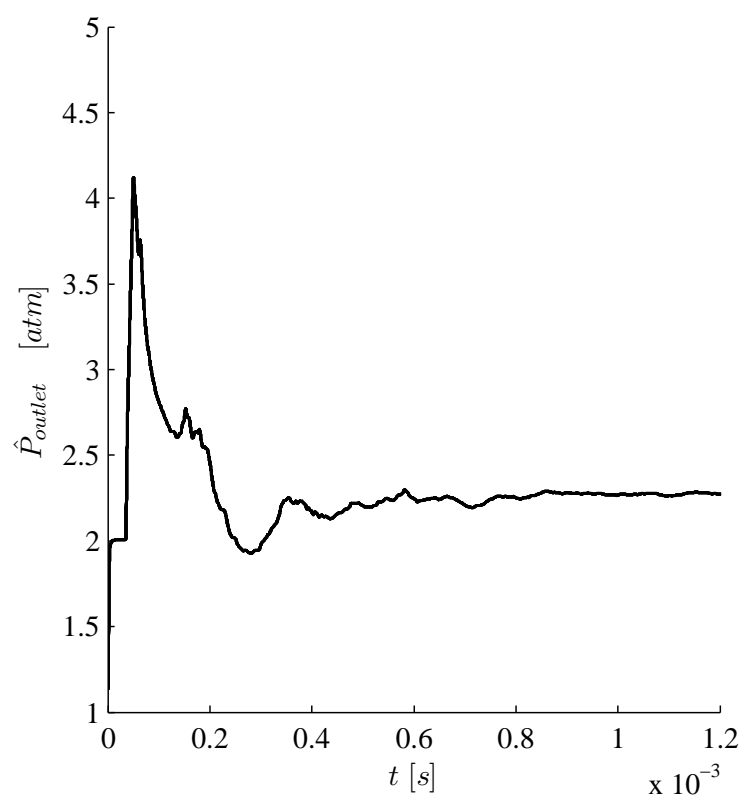

FIGURE 5.8: Outlet pressure prediction with $\sigma=-2 \pi$, well-imposed problem

Once the control of the mean flow pressure was guaranteed, a $C^{++}$subroutine was developed to evaluate the instantaneous value of the reflection coefficient $\left(R_{f}\right)$ at the outlet boundary. The developed function was implemented into the commercial CFD solver ANSYS-Fluent. The calculated local reflection coefficient is illustrated in Figure 5.9. It is observed that the predicted magnitude of the reflection coefficient is lower than 1 , for most of the region at the outlet.

It is possible to conclude that by adequately selecting the relaxation factor $(\sigma)$, the implementation of the NRBC and the LRM for RDC predictions successfully controls the mean flow pressure, and also reduces the reflection of the pressure waves from the outlet boundary. Therefore, the boundary condition model explained previously is implemented here on for the 


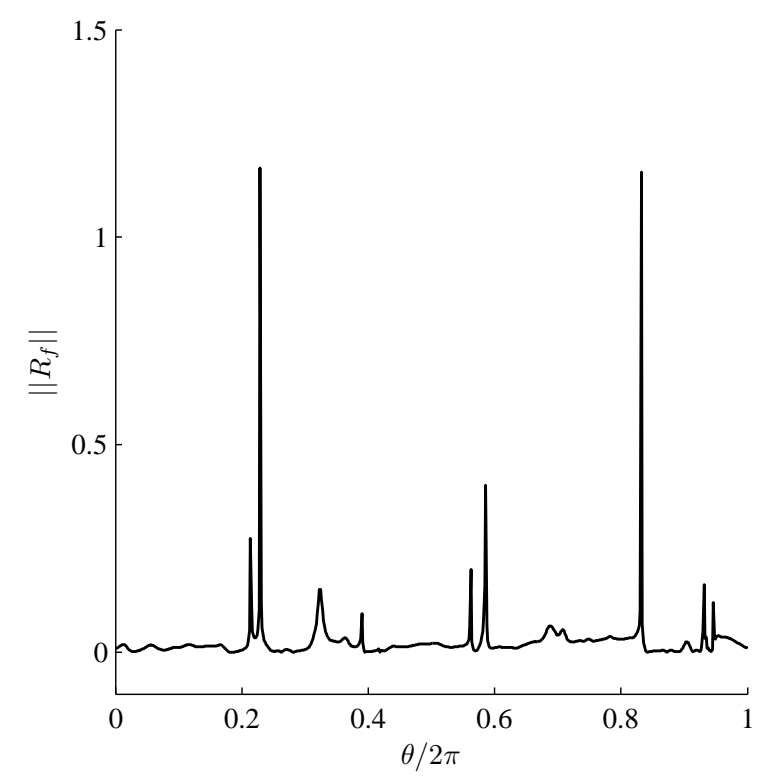

FIGURE 5.9: Instantaneous distribution of reflection coefficient for 2D RDC simulation with NRBC

prediction of RDC at elevated outlet conditions.

Two dimensional CFD simulations of RDC at elevated $P_{\infty}$ were performed. Three different injection geometries were considered, and values $P_{\infty}$ of up to 8.0atm were evaluated. The combustion chamber geometry selected for this section is based on the geometry described in Figure 4.15 . The original injection geometry was modified by increasing the air slot gap $\left(\Delta_{\text {air }}\right)$ in order to obtain different $A_{t} / A_{e}$ ratios. With an original value of 0.08 , the injection geometry was modified to obtain $A_{t} / A_{e}=0.2$ and 0.4 . The simulations were performed without modifying neither the combustion chamber geometric parameters $D_{i n}, D_{\text {out }}, L_{c}$ nor the injection parameters $P_{o, m}$ and $P_{o, m}$. On the other hand, $P_{\infty}$ was gradually increased. The increments of $P_{\infty}$ generally started from conditions of supersonic discharge of the detonation products, and was increased up to the limiting pressure when continuous detonation was no longer obtained. The temperature, velocity and density fields were characterized for each of the predicted conditions, and their corresponding performance parameters were calculated.

The first set of simulations presented in this section correspond to the original geometric configuration used as validation case in the current research. For this injection geometry $\left(A_{t} / A_{e}=0.08\right)$, four values of $P_{\infty}$ were studied $(0.11 \mathrm{~atm}, 1.0 \mathrm{~atm}, 2.0 \mathrm{~atm}$ and 3.0atm). Continuous detonation was achieved for $P_{\infty}=0.11,1.0$ and 2.0atm. Despite different initiation strategies implemented for the simulations with $P_{\infty}=2.5 \mathrm{~atm}$, the unidirectional detonation wave did not endure more than one complete cycle. The temperature contours illustrated in Figure 5.10, show that no appreciable differences are encountered when $P_{\infty}$ increases from $0.11 \mathrm{~atm}$ to 1.0atm. The characteristic flow structures in RDC are easily recognized for such results. The 
explanation given in Section 4.3.1 applies for $P_{\infty}=1.0 \mathrm{~atm}$. However, when $P_{\infty}$ increases to 2.0atm, an appreciable decrease in $\Delta_{D W}$ is observed. The evidence of vortical structures in the contact surface become apparent. The large range of temperatures in the temperature contours does not allow to appropriately appreciate the different flow structures and discontinuities. Hence, a better visualization is presented by the contours of the density gradients as shown in Figure 5.11.

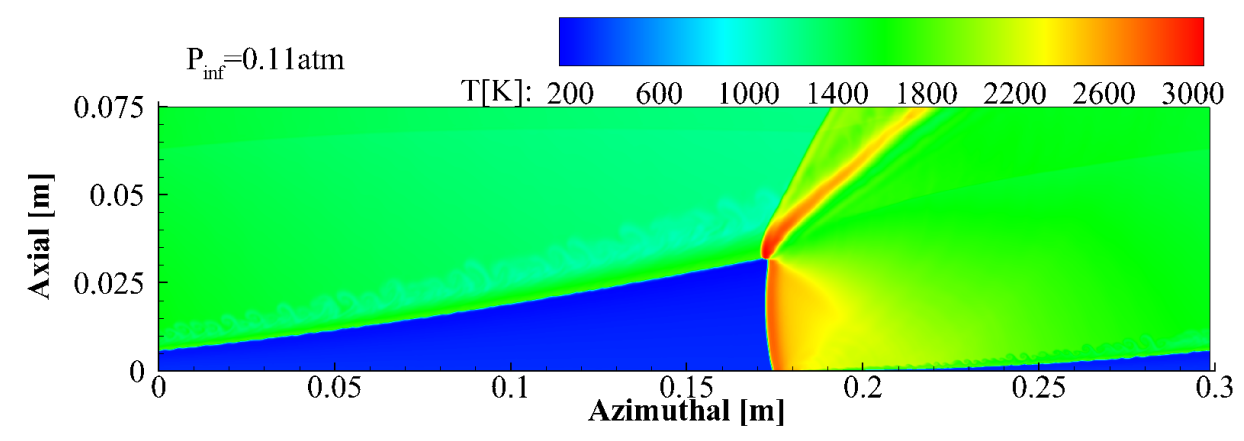

(a)

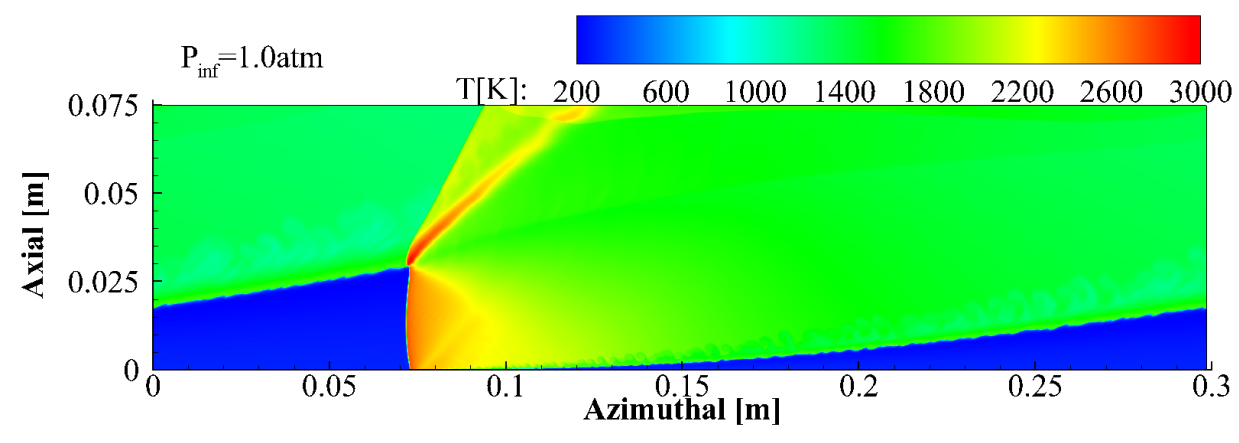

(b)

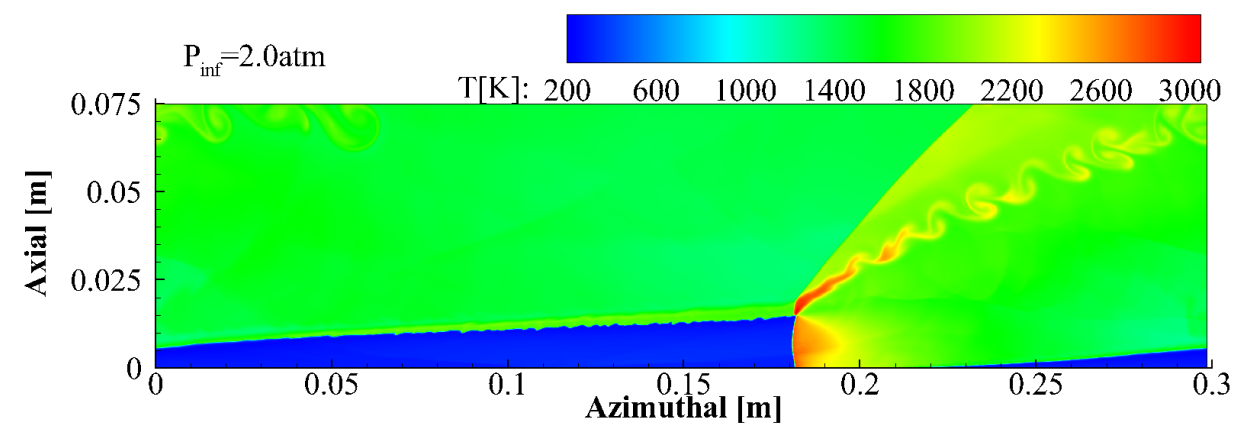

(c)

FIGURE 5.10: Temperature contours for 2D simulations of RDC with $A t / A e=0.08$ and $P_{\infty}=$ $0.11 \mathrm{~atm}(\mathrm{a}), 1.0 \mathrm{~atm}(\mathrm{~b})$ and $2.0 \mathrm{~atm}(\mathrm{c})$

From the density gradient contours, it is observed that the flow in RDC for $P_{\infty}=$ $0.11 \mathrm{~atm}$ and $1.0 \mathrm{~atm}$ does not present major differences. However, further examination reveals the presence of an azimuthal pressure wave near the outlet of the combustion chamber. This 
pressure wave is evidently stronger in the case with $P_{\infty}=1.0 \mathrm{~atm}$ than in $P_{\infty}=0.11 \mathrm{~atm}$, and it is not present for $P_{\infty}=2.0 \mathrm{~atm}$. When corroborated with the Mach number contours presented in Figure 5.12, it is possible to conclude that this oblique shock is responsible for slowing down the detonation products from a supersonic to a subsonic regime. The Mach number contours illustrated in Figure 5.12 and following Mach number contours, a dotted white line is added to depict the the solid line. The solid line separates the parts of the domain where the flow is subsonic from the supersonic region. The formentioned oblique shock wave "isolates" the detonation products from any upstream influence of the outlet boundary. Additionally, the eddy like structures forming along with the deflagration front are present for the simulations with $P_{\infty}$ equal to 0.11 and 1.0atms. The strength of the vortices decrease as they convect downstream in the axial direction of the combustion chamber, and have very little influence on the deflagration and on the detonation front.

In contrast, a more dramatic change in the flow structure in RDC is observed once $P_{\infty}$ is increased from 1.0atm to $2.0 \mathrm{~atm}$. Based on the density gradient contours Figure 5.11(c), it is evident that more complex flow structures are developed. First, the presence of a well defined azimuthal shock wave is no longer observed. When correlated with the Mach number contour plot in Figure 5.12(c), it is concluded that the reason is because the flow is subsonic in most of the region of the combustion chamber. In contrast with the simulations with lower $P_{\infty}$, the injected reactants do not reach supersonic conditions as they expand in the combustion chamber. The most evident change is observed in the behavior of the contact surface originated at the upper corner of the detonation front. It was discovered that partially reacted and hot combustion products are encountered by the shock front generated by the detonation wave. Due to the differences in density and temperatures of the gases before they meet the shock front, gradients in velocity and density result on their post shock state. These gradients lead to the onset of instabilities, similar to those formed near the deflagration front for lower pressure cases. Additionally, the density gradient present in the fresh mixture region corresponds to the pressure waves generated at the detonation front. More specifically, this applies to the waves that are generated at its upper corner. These pressure disturbances travel upstream in the subsonic flow and in the azimuthal direction within the expanding product. Eventually, such pressure waves interact with the deflagration front and the inlet region.

The distribution of pressure, temperature and Mach number at different axial locations of the combustion chamber was sampled as a function of time in order to average certain performance parameters. The instantaneous stagnation pressure and Mach number distribution at the outlet of the combustion chamber for the injection geometry with $A_{t} / A_{e}=0.08$ at different $P_{\infty}$ are presented in Figure 5.13. The distribution of Mach number confirms the observation made previously, that the flow regime at the outlet changes with the far field pressure. It is observed that supersonic, mixed subsonic and supersonic, and fully subsonic outlet conditions are encountered for $P_{\infty}$ equal to $0.11,1.0$ and 2.0 atm, respectively. In regards to the total pressure, it 


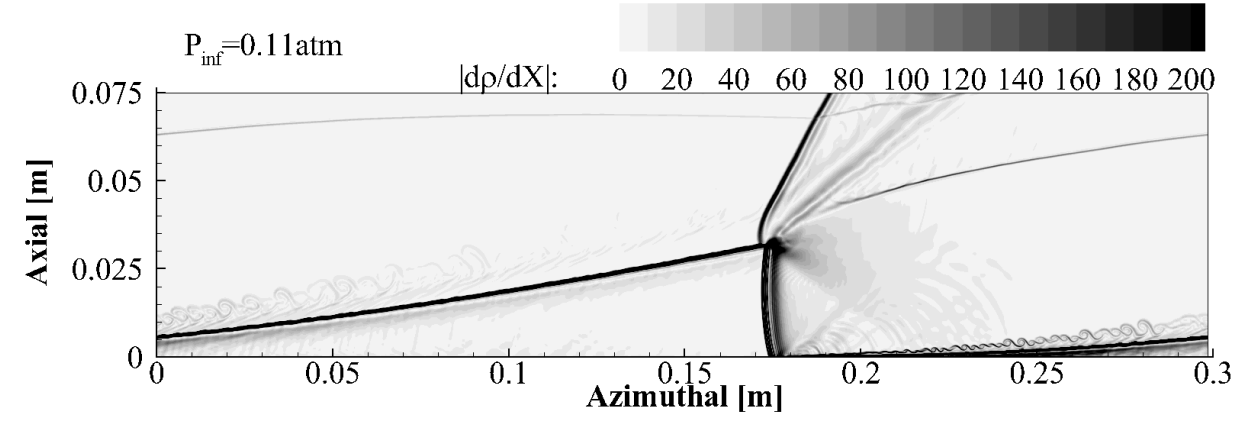

(a)

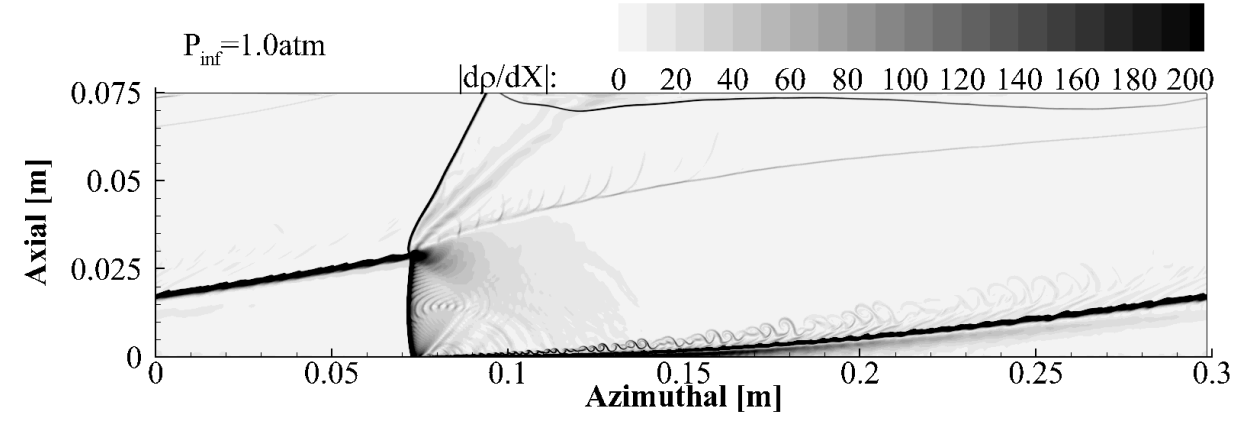

(b)

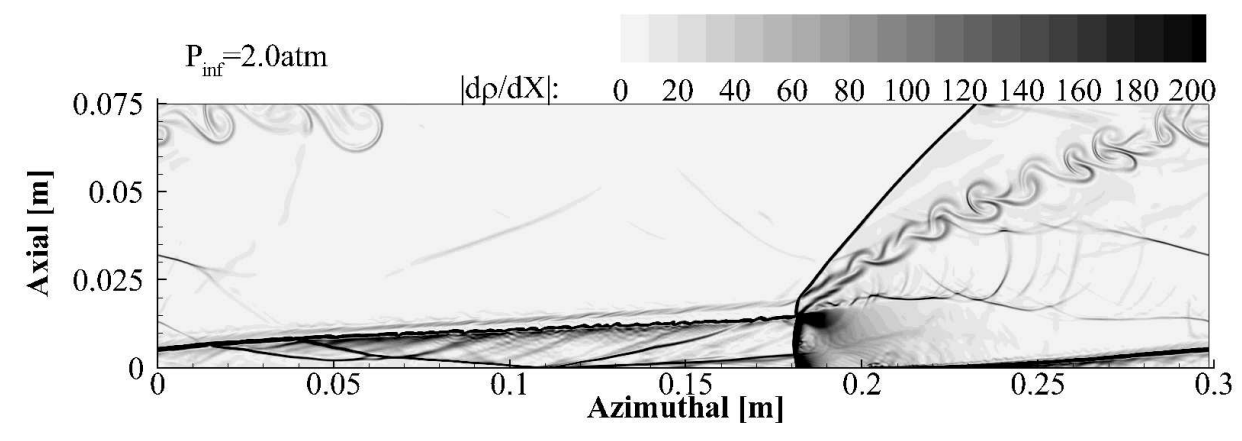

(c)

FIGURE 5.11: Density gradient contours for 2D simulations of RDC with $A t / A e=0.08$ and $P_{\infty}=0.11 \mathrm{~atm}(\mathrm{a}), 1.0 \mathrm{~atm}(\mathrm{~b})$ and 2.0atm(c)

is observed that the total pressure distribution decreases when $P_{\infty}$ increases from 0.11 to $1.0 \mathrm{~atm}$. This is due to the presence of a strong azimuthal shock wave depicted in Figure 5.11. However, once $P_{\infty}$ increases from 1.0 to $2.0 \mathrm{~atm}$ the total pressure distribution increases throughout the outlet boundary. Such behavior can be explained due to the absence of the shock wave in the mostly subsonic flow.

The location of the reaction front was also obtained from the 2D CFD simulations. The reaction surface was defined as the region in which $50 \%$ of the reactants have been consumed. The change in the shape of the deflagration front due to the increase in $P_{\infty}$ is presented in 


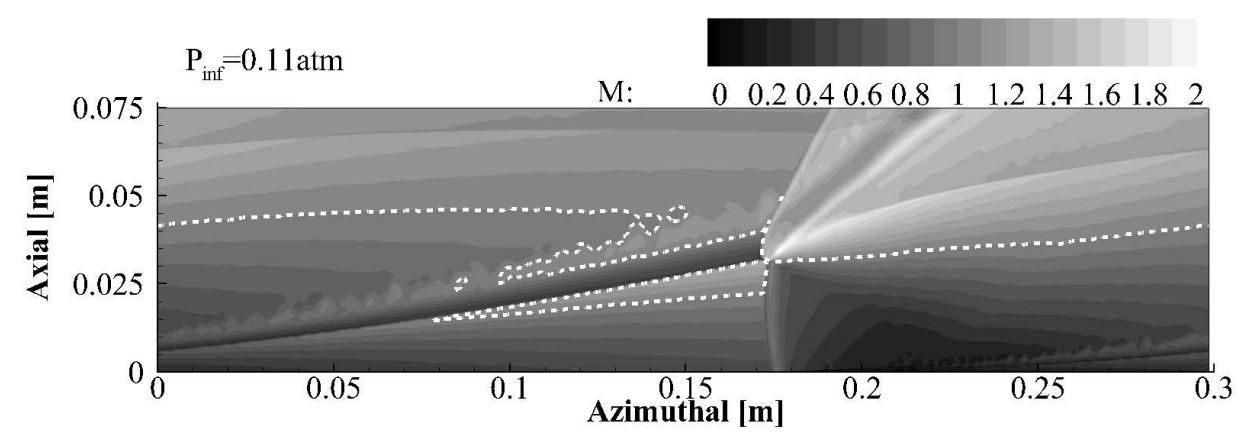

(a)

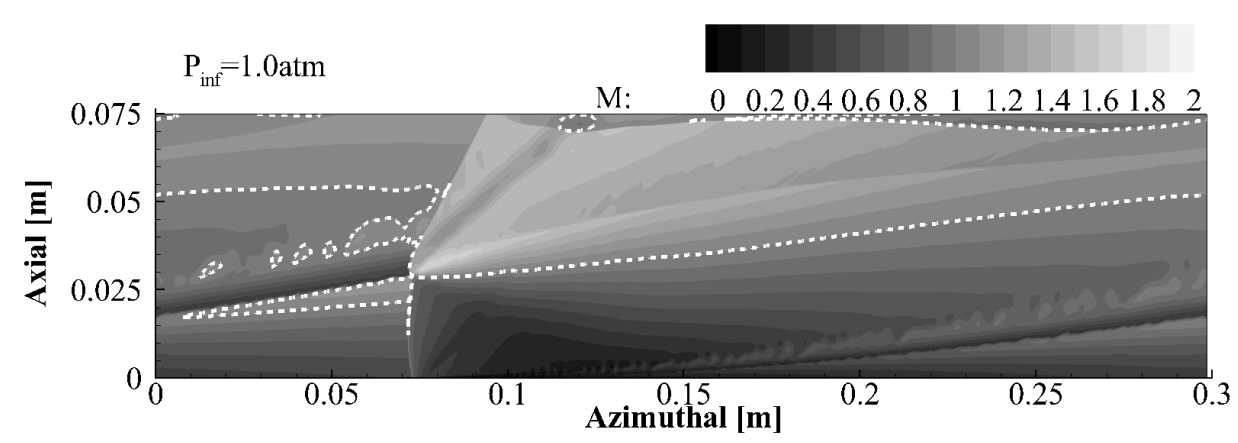

(b)

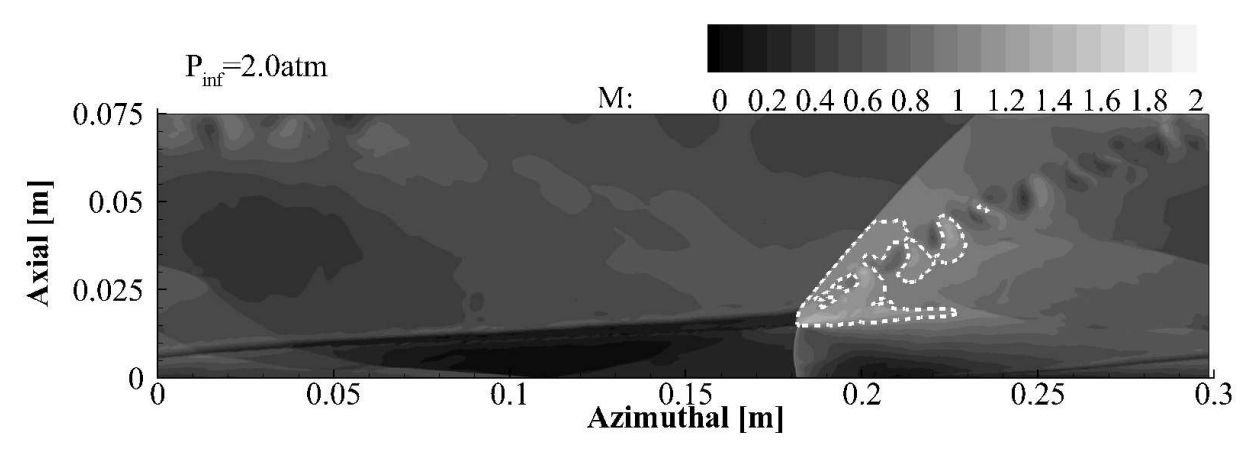

(c)

FIGURE 5.12: Mach number contours for 2D simulations of RDC with $A t / A e=0.08$ and $P_{\infty}=$ $0.11 \mathrm{~atm}(\mathrm{a}), 1.0 \mathrm{~atm}(\mathrm{~b})$ and 2.0atm(c). (White dotted line represents the contour line for $\mathrm{M}=1$ )

Figure 5.14. The deflagration front shape and the detonation front height does not present substantial change when $P_{\infty}$ increases from 0.11 to 1 .0atm. On the other hand, $\Delta_{D W}$ decreases from $29.7 \mathrm{~mm}$ to $14.9 \mathrm{~mm}$ when $P_{\infty}$ increases from 1.0 to $2.0 \mathrm{~atm}$. Such behavior can be explained by the difference in the flow regimes for $P_{\infty}=1.0$ and 2.0atm. As illustrated in Figure 5.12(b), a large portion of the the flow in the 2D domain is supersonic; whereas for $P_{\infty}=2.0 \mathrm{~atm}$, the flow is mostly subsonic. As summarized in Table 5.1, $\Delta_{D W}$ decreases substantially once the change in the flow regime occurs. The value of $P_{\infty}$ at which the majority of the flow inside the combustion chamber changes from supersonic to subsonic is a function of the injection geometry, meaning that for the current study it varies with the parameter $A_{t} / A_{e}$. 


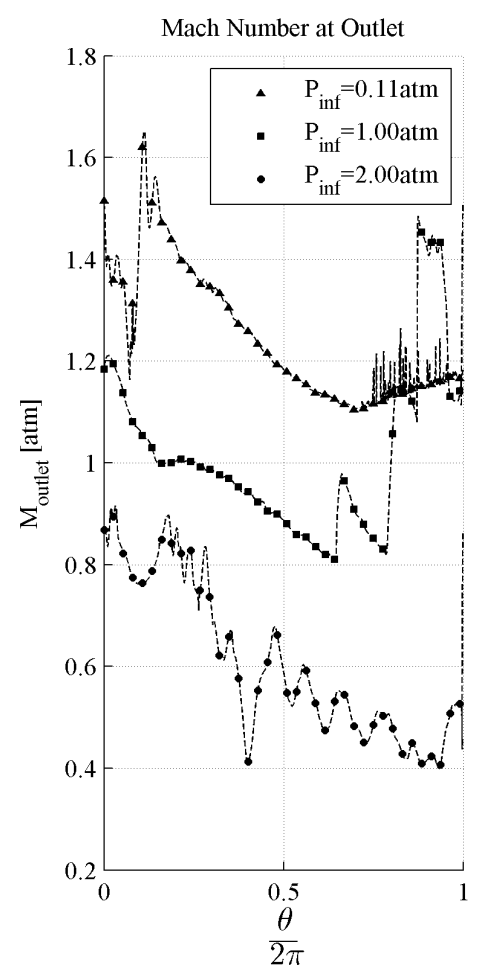

(a)

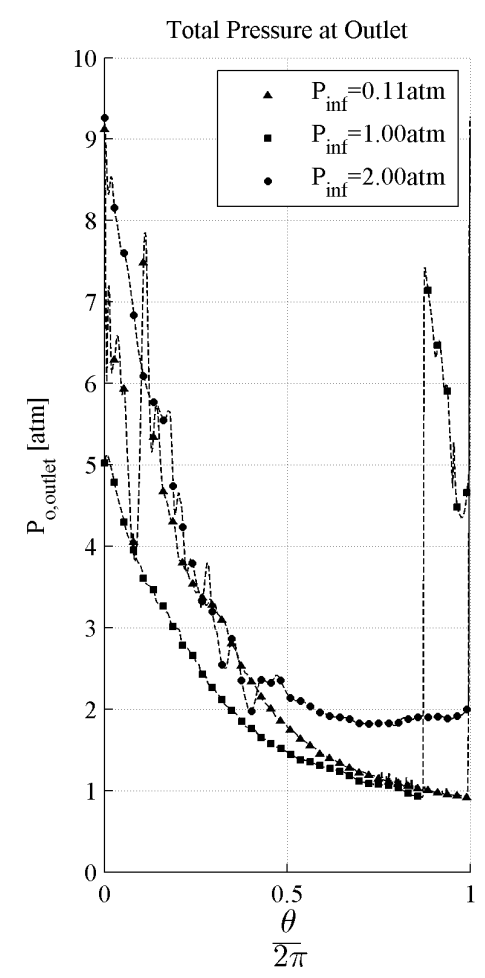

(b)

FIGURE 5.13: Mach number (a) and stagnation pressure (b) distribution at the combustion chamber outlet for $A t / A e=0.08$ and $P_{\infty}=0.11,1.0$ and $2.0 \mathrm{~atm}$

It is observed that $\Delta_{D W}$ decreases when $P_{\infty}$ increases, but no perceptible change in $\theta_{r}$ was observed.

The second set of 2D simulations for RDC at elevated $P_{\infty}$ were performed for an injection geometry with $A_{t} / A_{e}=0.2$. Five values of f $P_{\infty}$ were evaluated under this injection configuration: $0.11,1.0,2.04 .0$ and 6.0atm. Continuous detonation was obtained for $P_{\infty}$ equal and lower than $6.0 \mathrm{~atm}$. Despite the efforts to establish continuous detonation for $P_{\infty}=6.5$ and 7.0atm, the detonation wave did not exist for more than one cycle. In the attempts where the continuous detonation was not achieved, the combustion regime transitioned from an initialized detonation state into a deflagration regime. It was identified that the there was not sufficient time for the reactants to enter into the combustion chamber before the detonation wave completed one cycle.

The results obtained for $P_{\infty}=0.11$ and 1.0atm, led to continuous detonation and presented supersonic exhaust of the detonation products. The temperature, Mach number and density gradient distribution in the combustion chamber follows that of the explained for Figure 5.10(a). For such values of $P_{\infty}$ there is no strong azimuthal shock waves or significant oscillations of the detonation front. The contour plots for $P_{\infty}=0.11$ and $1.0 \mathrm{~atm}$ are not presented 
Detonation and combustion front

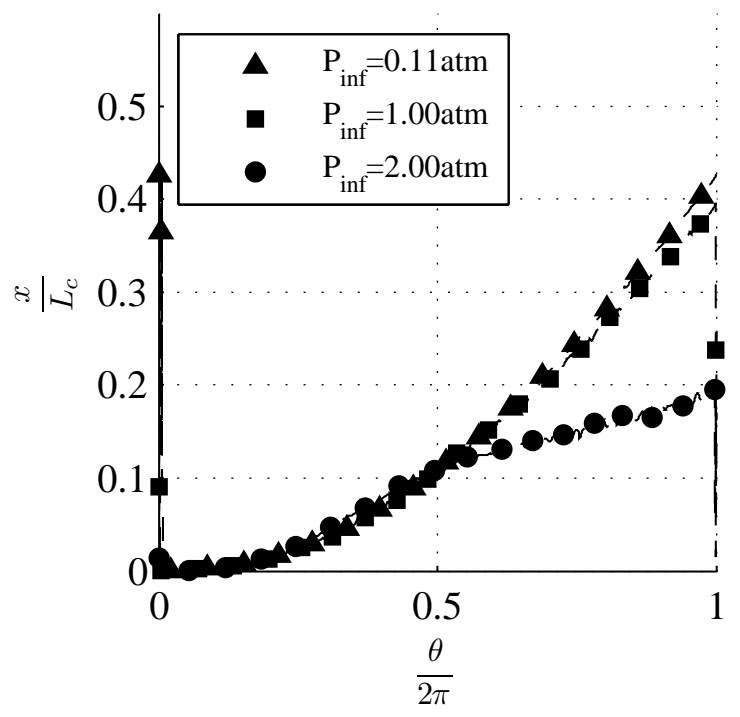

FIGURE 5.14: Prediction of deflagration front shape from 2D RDC simulation with $A_{t} / A_{e}=0.08$ at elevated pressures

in this document, such regime has been previously covered. Instead, the interest is focused for operating conditions leading to mixed or fully subsonic exhaust velocities.

A strong pressure wave located near the combustion chamber outlet is observed for $A_{t} / A_{e}=0.4$ and $P_{\infty}=2.0 \mathrm{~atm}$, see Figure 5.17( $\mathrm{a}$ and $\mathrm{b}$ ). The shock wave covers the complete circumferential span of the combustion chamber, and it is responsible for slowing down the detonation products from supersonic to subsonic velocities. The influence of this strong wave is also evident in the density gradient contours (Figure 5.15(a)) and the temperature contours (Figure 5.15(b)). In the former it is illustrated as a high density gradient region, while in the latter it increases the temperature of the detonation products near the outlet boundary. Such effect is more evident on the high temperature gases flowing between the contact surface and the oblique shock wave. In the case of $P_{\infty}=2.0 \mathrm{~atm}$ the presence of a strong shock wave reducing the velocity of the detonation products from approximation $\mathrm{M}=2.0$ to subsonic condition is a detriment to the performance of RDC, since it induces losses in total pressure.

Once $P_{\infty}$ increases to $6.0 \mathrm{~atm}$, a more chaotic flow behavior in the combustion chamber is observed. First, the temperature contour in Figure 5.15(c) shows how the deflagration front height decreases, the corrugation of the deflagration front increases and the temperature fluctuations in the contact surface are stronger. The deflagration front is corrugated and eddies are formed behind the flame front. Instead of decreasing in strength, the vortices appear to increase as they are convected downstream in the combustion chamber. Once these structures meet the oblique shock wave pressure oscillations behind the detonation front are created. 


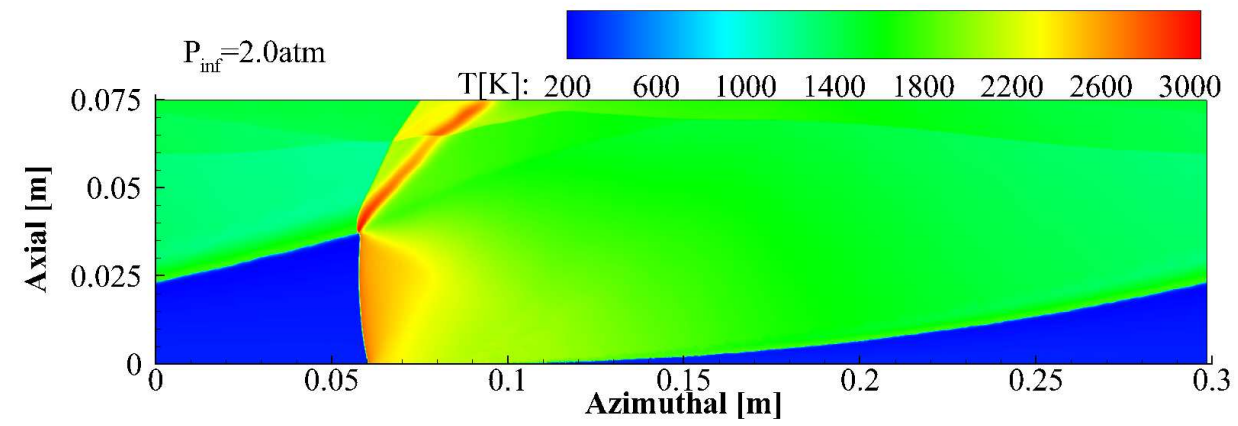

(a)

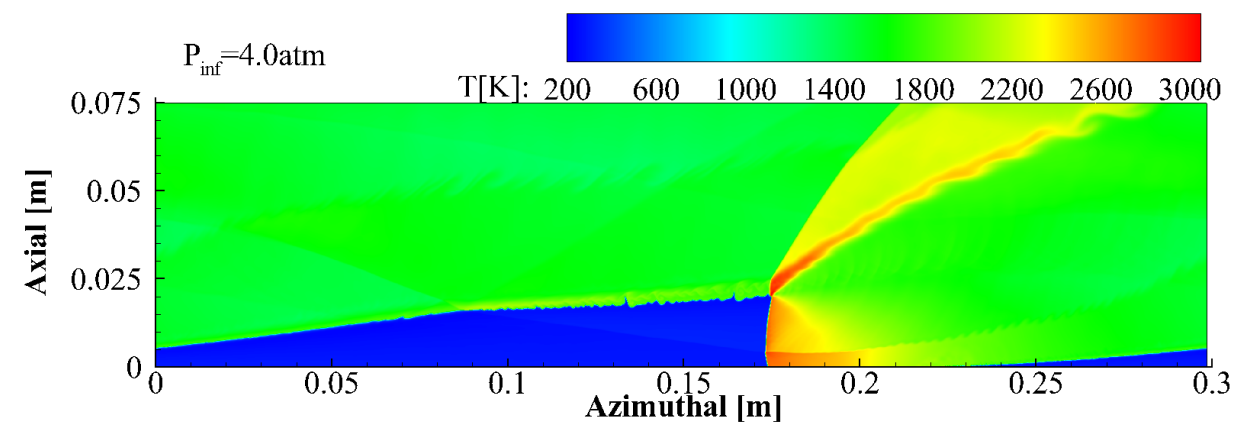

(b)

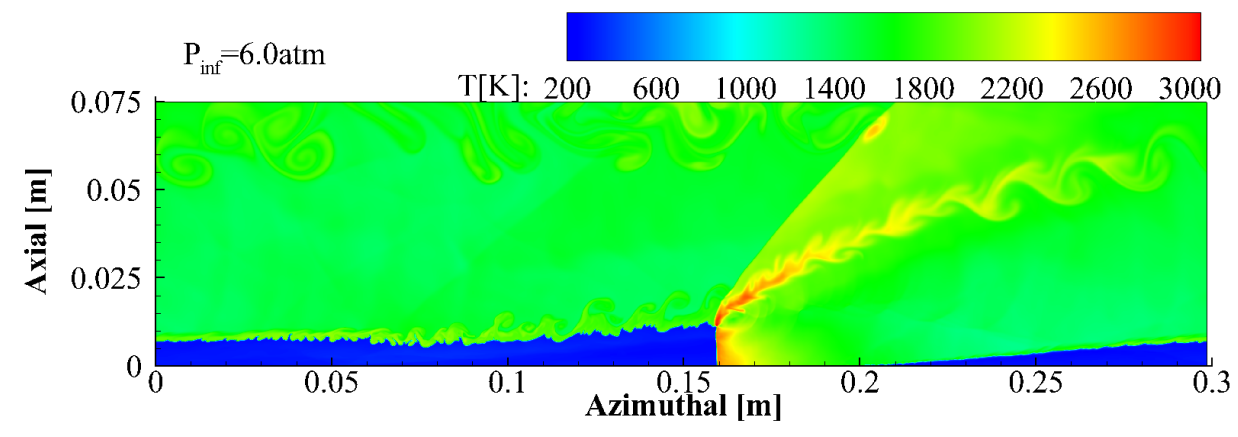

(c)

FIGURE 5.15: Temperature contours for 2D simulations of RDC with $A t / A e=0.2$ and $P_{\infty}=$ 2.0 atm (a), 4.0atm (b), and 6.0atm(c)

The detonation product regime in the combustion chamber changes dramatically when the $P_{\infty}$ is increased from 2.0 to $4.0 \mathrm{~atm}$. The regions of supersonic flow at $2.0 \mathrm{~atm}$ are no longer present for the simulation with 4.0atm (see Figure 5.17(a)(b)). The subsonic nature of the flow at $P_{\infty}=4.0 \mathrm{~atm}$ leads to upstream influence of pressure fluctuation into the inlet, the deflagration front and the detonation wave. From the temperature and density gradient contour plots (Figure 5.15(b) and Figure 5.16(b)) it is observed that the deflagration front presents a small level of corrugation. Despite the small scale of such fluctuation, they have an important effect on the flow behind the detonation front. It is observed that if the detonation front encounters an irregular deflagration front pressure fluctuations are generated at its upperr corner. Second, 


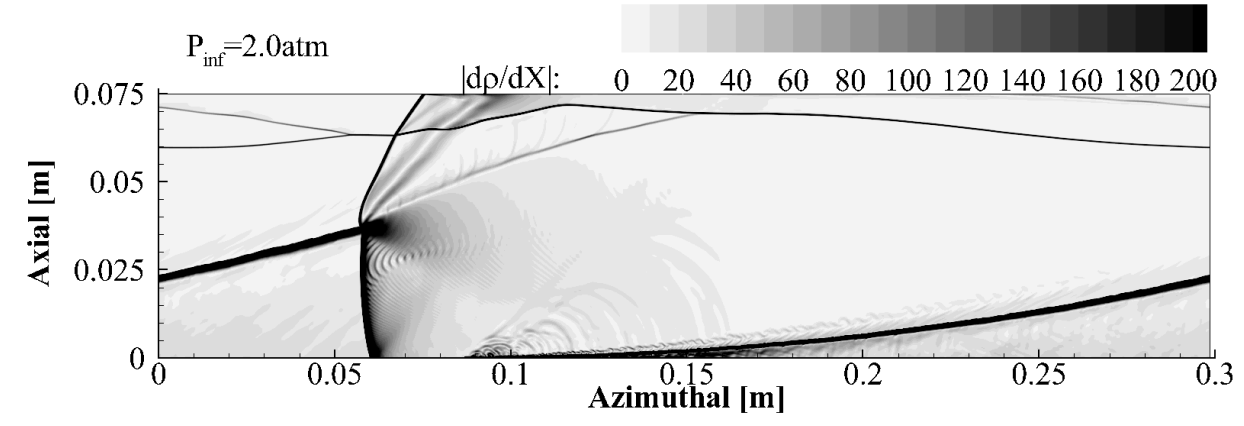

(a)

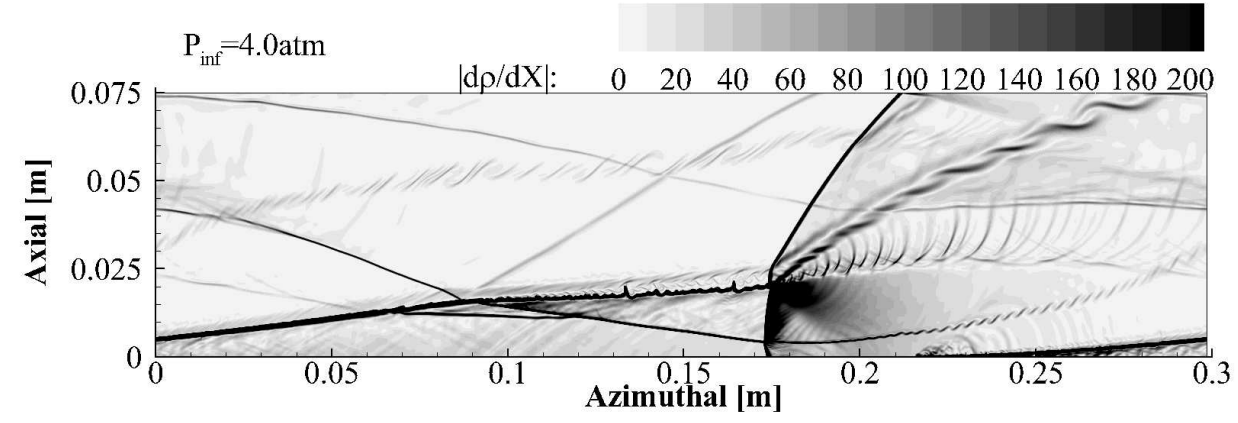

(b)

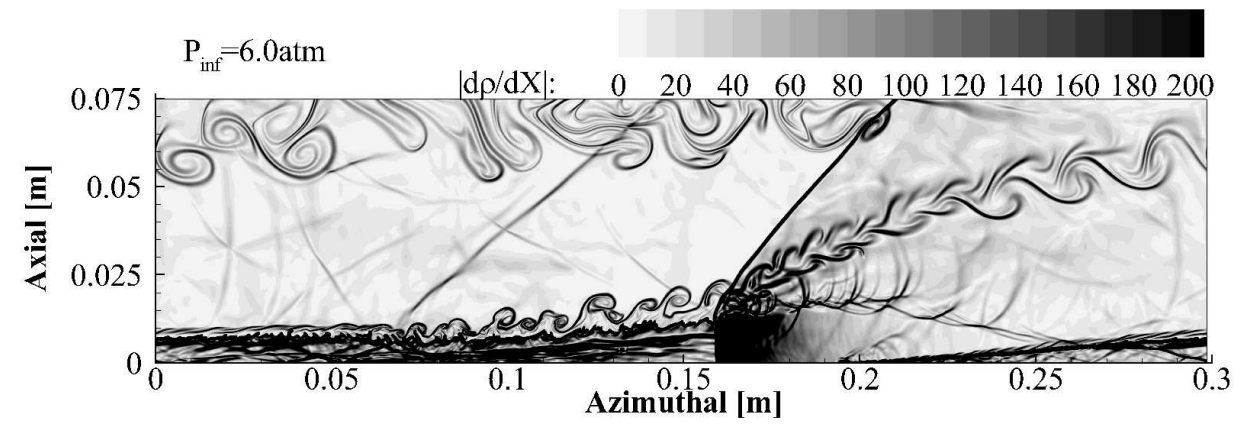

(c)

FIGURE 5.16: Density gradient contour plots for 2D simulations of RDC with $A t / A e=0.2$ and $P_{\infty}=2.0 \mathrm{~atm}(\mathrm{a}), 4.0 \mathrm{~atm}(\mathrm{~b})$, and 6.0 atm(c)

the density gradient plots illustrated in Figure 5.16(c) evidence the presence of strong vortical structures generated from the detonation and the deflagration front. The interrelation and self sustaining nature of these structures, as described previously, is evident. The presence of strong fluctuations at elevated outlet pressure narrows the far field pressure range at which continuous detonation can be achieved.

The influence of the far field pressure is evident in the deflagration front shape and the detonation wave height. Figure 5.18 illustrates these variations for $A_{t} / A_{e}=0.2$ for different values of $P_{\infty}$. Supporting the previous descriptions, Figure 5.18 shows how the deflagration 


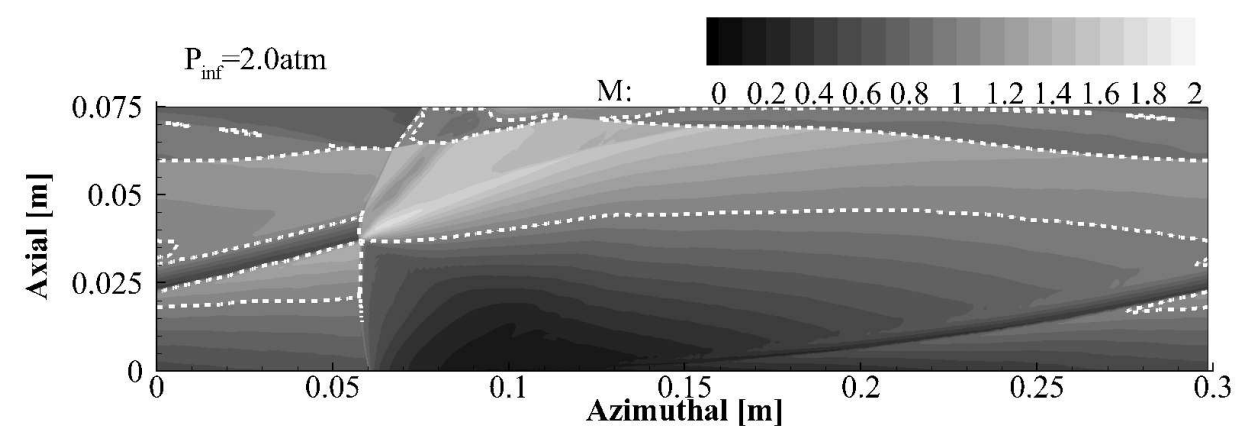

(a)

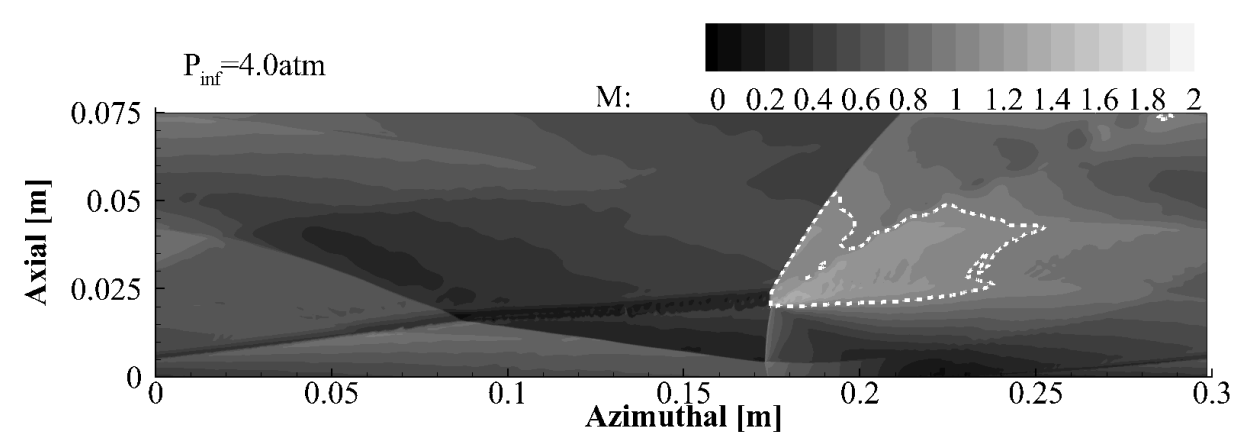

(b)

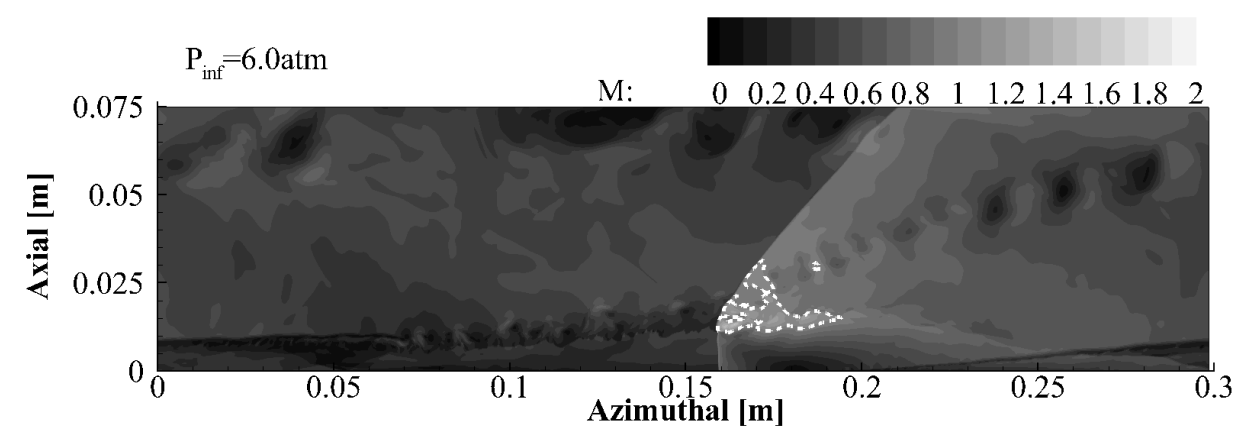

(c)

FIGURE 5.17: Mach number contour plots for 2D simulations of RDC with $A t / A e=0.2$ and $P_{\infty}=2.0 \mathrm{~atm}$ (a), 4.0 atm (b), and 6.0 atm(c) (White dotted line represents the contour line for $M=1$ ). (White dotted line represents the contour line for $M=1$ )

front remains almost unaltered for far field pressures between 0.11atm and 2.0atm. However $\Delta_{D W}$ decreases almost by $50 \%$ when $P_{\infty}$ increases from 2.0 to $4.0 \mathrm{~atm}$. 


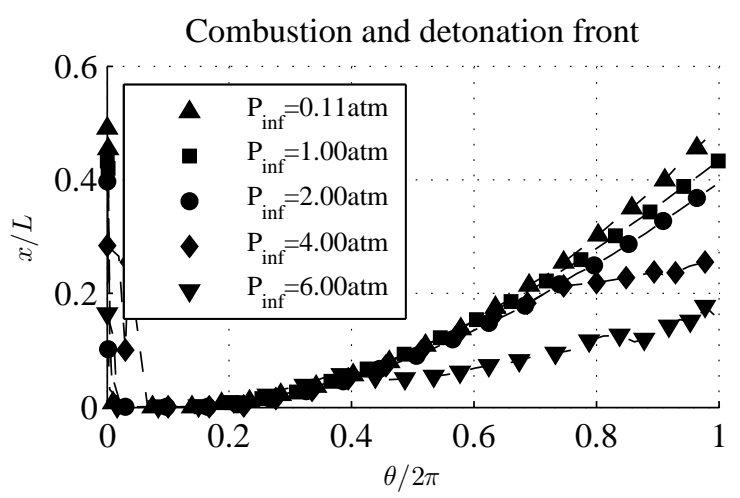

FIGURE 5.18: Prediction of deflagration front shape from 2D RDC simulation with $A_{t} / A_{e}=0.2$ at elevated pressures

The third group of 2D simulations was performed for an injection geometry with an $A_{t} /$ Ae ratio equal to 0.4. The simulations showed that for $P_{\infty}$ equal to 4.0, 6.0, 8.0 and $8.5 \mathrm{~atm}$, a continuous detonation regime can be achieved. However once $P_{\infty}$ was increased to 9.0atm, unstable operation was predicted. For instance, if $P_{\infty}=9.0 \mathrm{~atm}$ a regime oscillating between deflagration and detonation events was observed. Under such unstable case, the detonation wave front was not large enough to endure more than two continuous cycles. The injected reactants would not reach an axial distance sufficient to support the continuous propagation of the detonation front. However, once the detonation front vanished, the reactants would continue to be consumed by the flame front, but in the presence of a strong pressure wave in the combustion chamber. The presence of the pressure wave would then re-ignite the gases at a new front and lead to the reappearance of a detonation wave. Such behavior was observed for no more than two instances, and then the detonation wave would vanished completely leaving only the deflagration front.

For the conditions leading to continuous detonation with the injection geometry $A_{t} / A e=$ 0.4, the simulations showed a similar pattern to those described for $A_{t} / A e=0.08$ and 0.2 . The contour of temperature, density gradient and Mach number are presented in the series of illustrations Figure 5.19, Figure 5.20 and Figure 5.21, which show the results for $P_{\infty}=$ 6.0, 8.0 and $8.5 \mathrm{~atm}$. It is, once again, observed that the oscillations in the deflagration and the detonation front are generated as $P_{\infty}$ is increases. Similarly it is corroborated that the fluctuations are more intense when the Mach number in most of the combustion chamber is smaller than 1.

For $P_{\infty}=8.5 \mathrm{~atm}$, the pressure waves generated at the upper corner of the detonation front have a strong influence in the injection conditions and lead to the corrugation of the deflagration front. The presence of vortical structures is also captured in the vicinity of the deflagration front and the contact surface. 


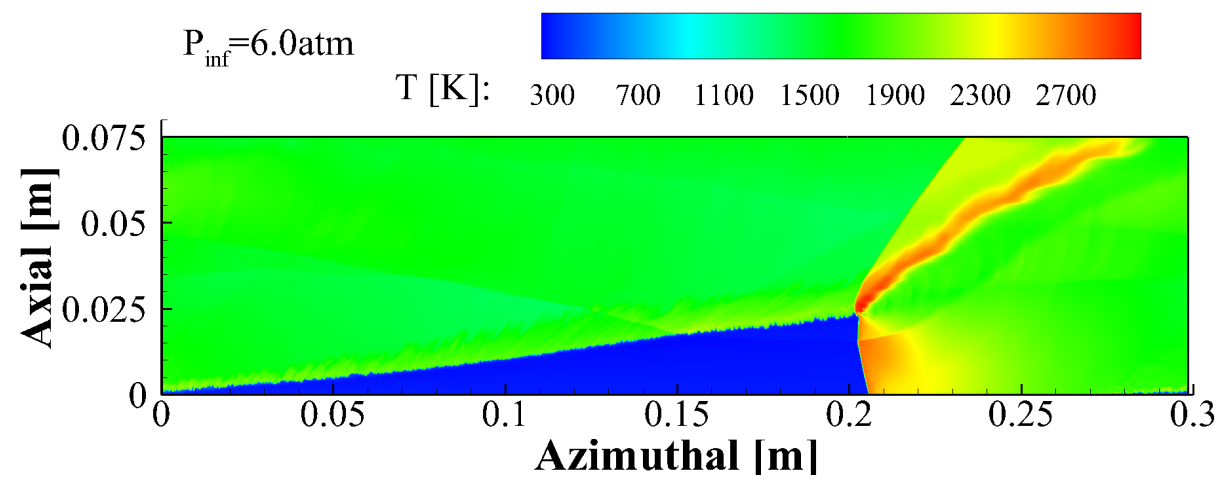

(a)

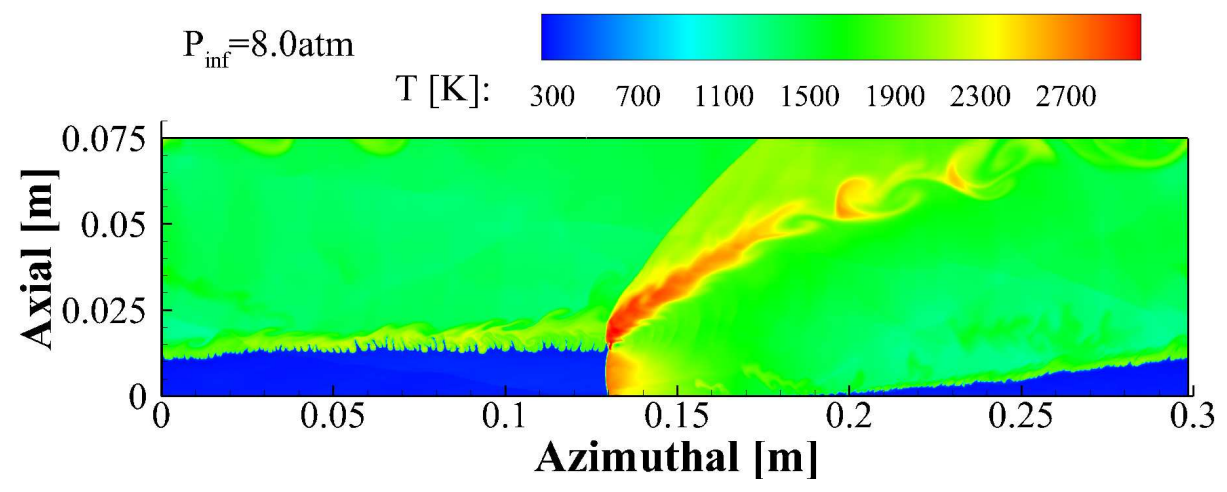

(b)

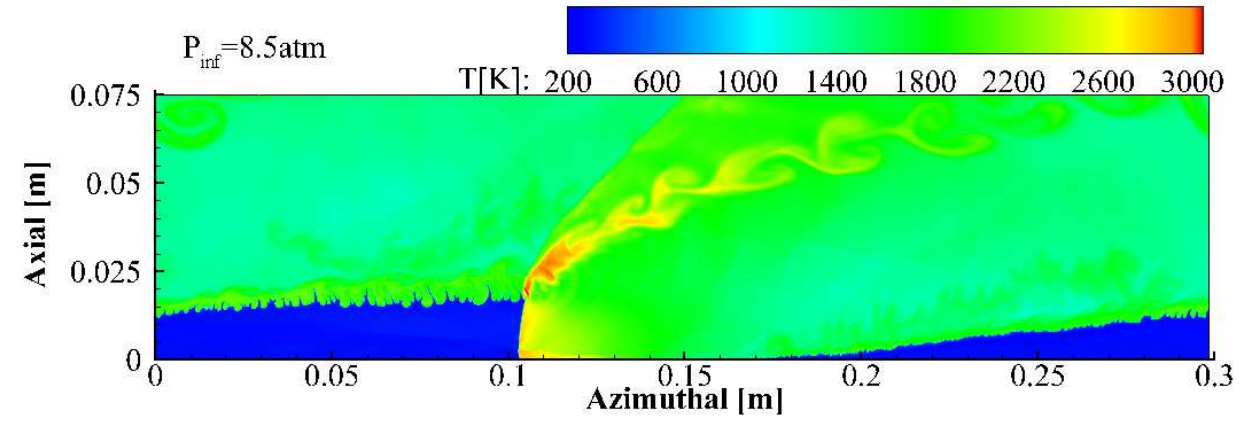

(c)

Figure 5.19: Temperature contour plots for 2D simulations of RDC with $A t / A e=0.4$ and $P_{\infty}=6.0 \mathrm{~atm}(\mathrm{a}), 8.0 \mathrm{~atm}(\mathrm{~b})$, and 8.5atm(c) 


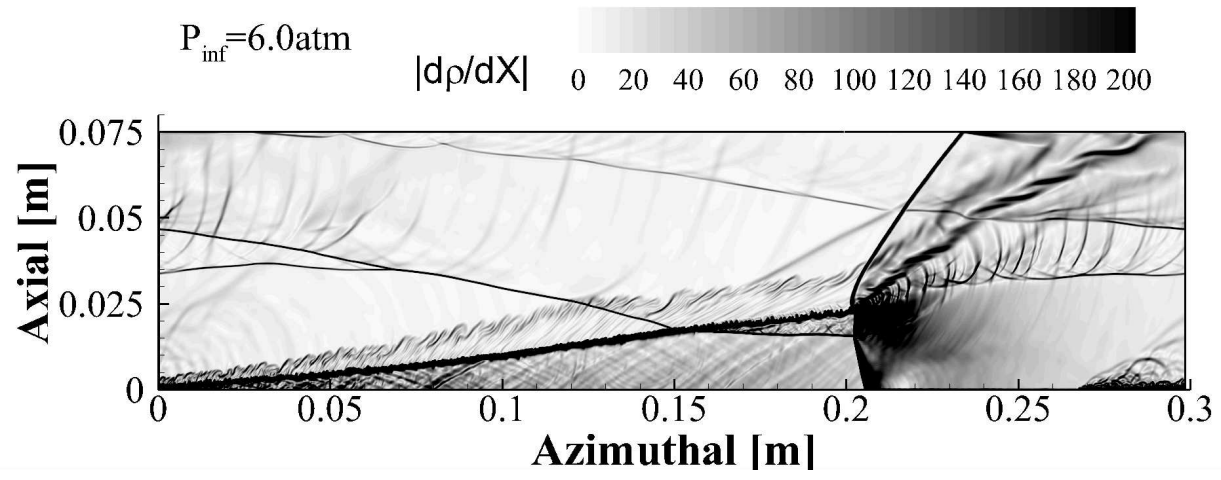

(a)

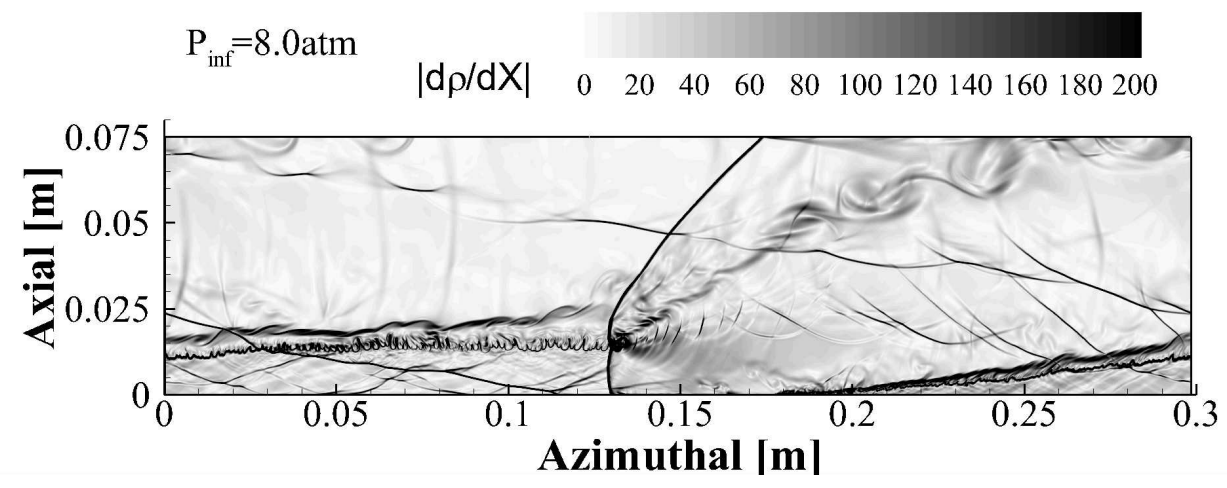

(b)

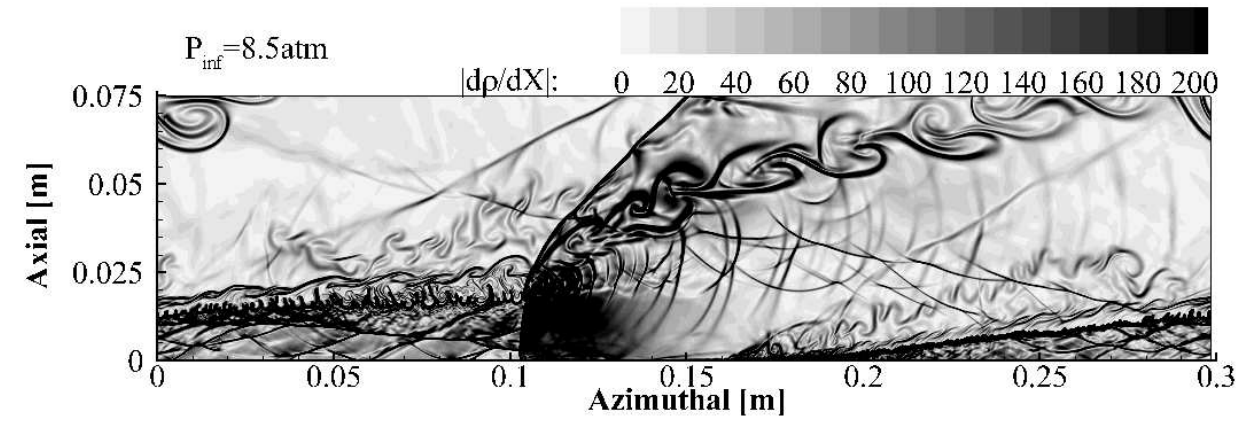

(c)

Figure 5.20: Density gradient contour plots for 2D simulations of RDC with $A t / A e=0.2$ and $P_{\infty}=6.0 \mathrm{~atm}(\mathrm{a}), 8.0 \mathrm{~atm}(\mathrm{~b})$, and $8.5 \mathrm{~atm}(\mathrm{c})$ 


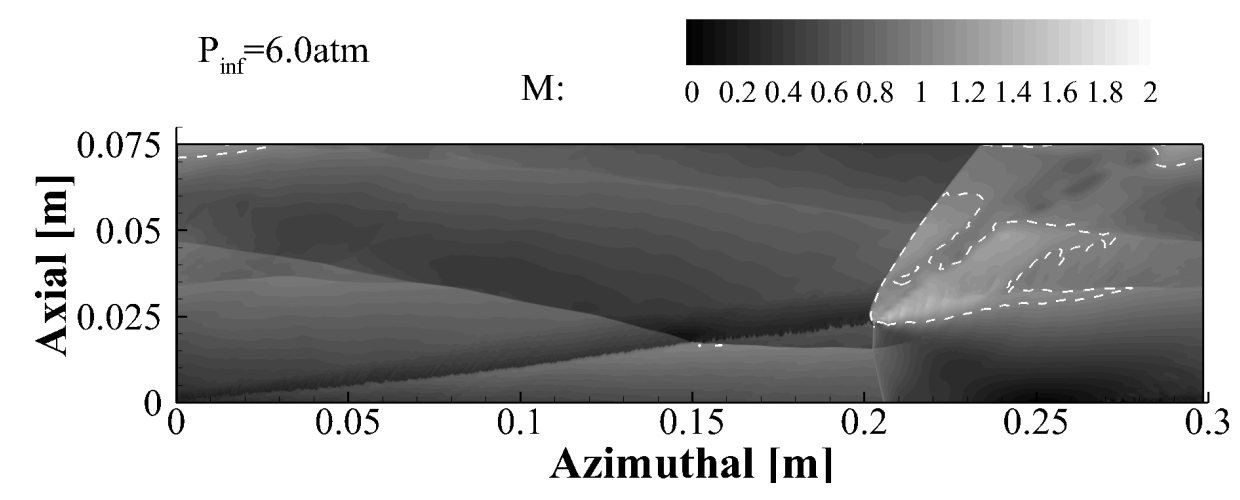

(a)

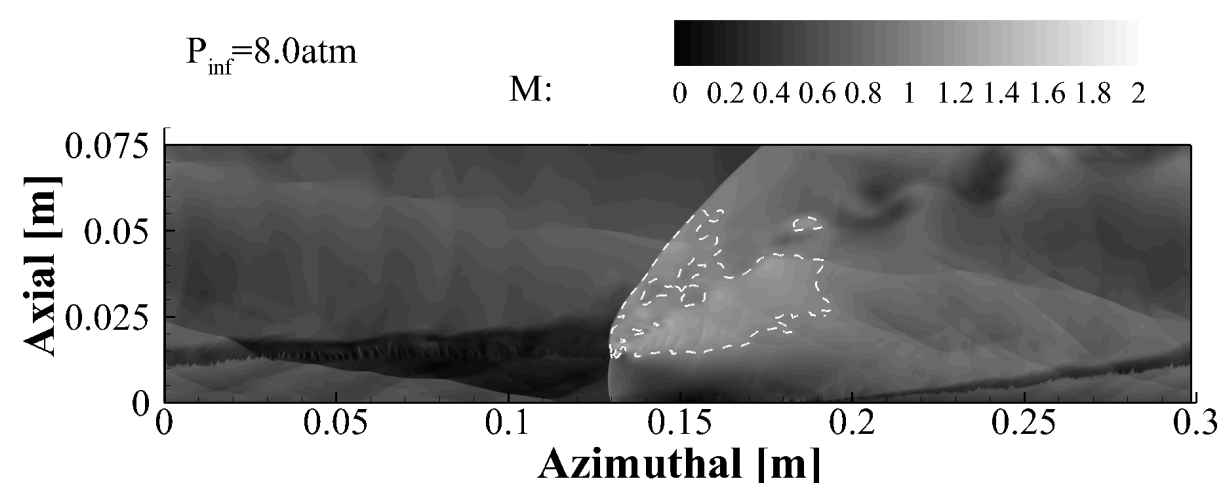

(b)

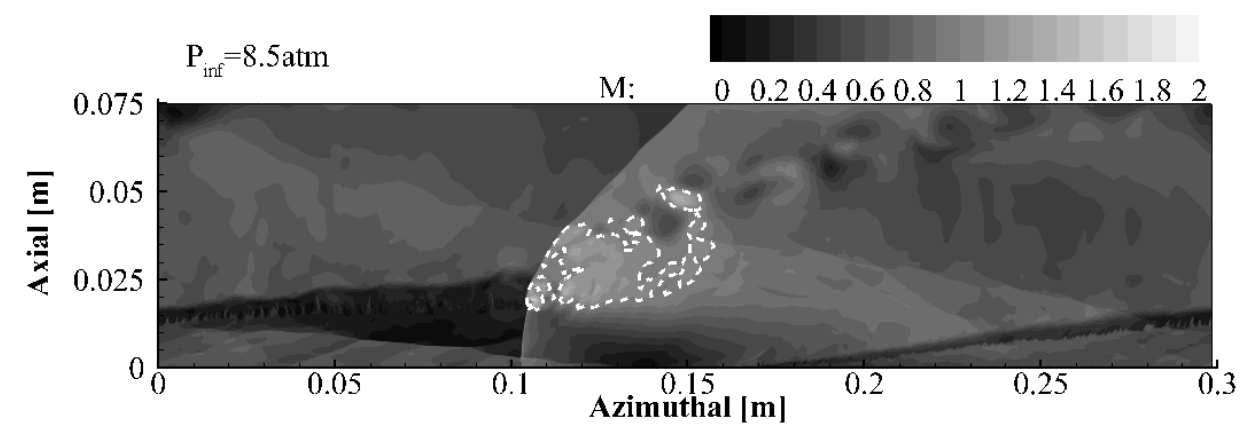

(c)

FIGURE 5.21: Mach number contour plots for 2D simulations of RDC with At/Ae $=0.4$ and $P_{\infty}=6.0 \mathrm{~atm}(\mathrm{a}), 8.0 \mathrm{~atm}(\mathrm{~b})$, and 8.5atm(c)(White dotted line represents the contour line for $\mathrm{M}=1$ ) 
Based on the previous observations, it is possible to define three characteristic regimes in which RDC can occur:

The first regime is defined as that in which the exhaust of the detonation product occurs at supersonic conditions in the entire span of the outlet surface. Under such operation conditions, the gases accelerate to supersonic velocities at an axial distance approximately equal to the detonation wave height, as illustrated in Figure 5.12(a). Despite the presence of eddies formed downstream of the deflagration and detonation front, the flow is relatively stable in its periodic nature.

The second regime corresponds to that where the detonation products accelerate to supersonic velocities at an axial distance also close to the detonation wave height, but are then decelerated by the presence of an azimuthal shock wave to subsonic velocities. This behavior is observed in the cases of $A_{t} / A_{e}=0.08$ and $P_{\infty}=1.0$ and $A_{t} / A_{e}=0.2$ and $P_{\infty}=2.0$. The presence of the shock wave in the circumference of the combustion chamber is caused by the increased far field pressure, and it leads to additional losses in the total pressure of the detonation products.

The third regime corresponds to that where the Mach number of the gases in the combustion chamber is predominantly lower than one. The supersonic region is then confined to a small triangular area formed between the oblique shock wave, the contact surface and the upper corner of the detonation front. This regime is characterized by the presence of strong vortical structures that appear near the deflagration zone and are amplified when they interact with the detonation wave and oblique shock wave.

After characterizing the behavior of RDC at elevated far field pressures, it is possible to compare the results for the multiple geometrical configurations and far field pressure. The comparison is achieved based on different integral performance parameters such as $U_{D W}, \Theta, \dot{m}$ ” and $P_{o, l o s s}$.

As illustrated in Figure 5.22, it is observed that the detonation wave velocity increases as $P_{\infty}$ increases. This results appropiattely correlate to the $\mathrm{CJ}$ calculations lillustrated in Section 2.1. More over, it was obtained that there is no influence in the detonation wave velocity due to the different injection configurations. It is known that for a fixed geometrical configuration of the combustion chamber, the increase in detonation wave velocity is to the detriment of continuous detonation. However, the increment in $U_{D W}$ is of only $5 \%$ and it does not play a governing role in the limitation for continuous detonation.

If the mass flux at the outlet of the combustor $\dot{m}_{\text {outlet }}$ is studied, it is obtained that the mass flux increases when the area ratio $A_{t} / A_{e}$ increases, see Figure 5.23. This behavior is expected, as it was previously explained and justified by Equation 3.12. Additionally, it is 


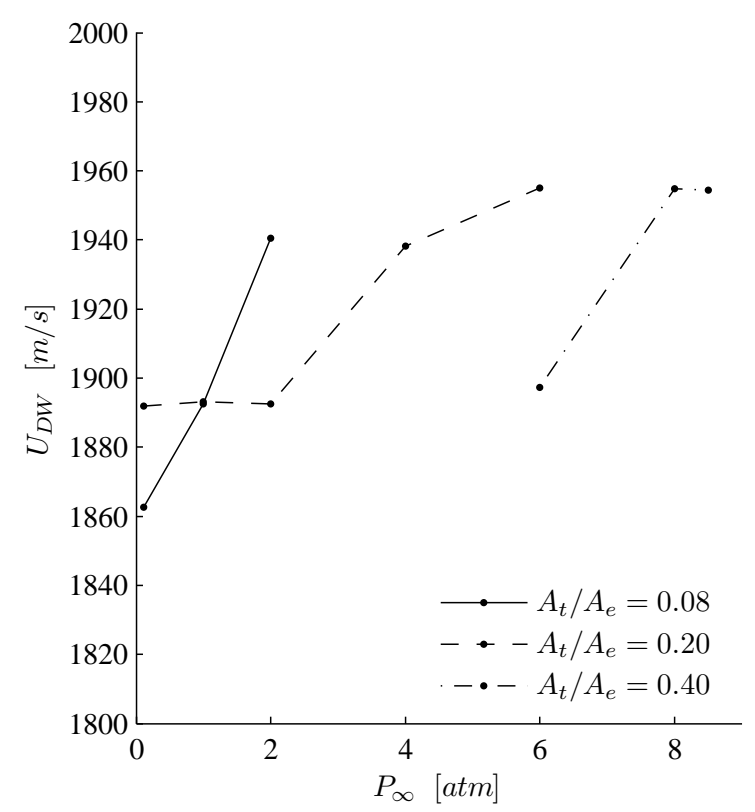

FiguRE 5.22: Prediction of detonation wave velocity from 2D RDC simulation with $A_{t} / A_{e}=$ $0.08,0.02$ and 0.4 at elevated pressures

observed that once $P_{\infty}$ increases $\dot{m}_{\text {outlet }}$ is initially increased but it then decreases, except for $A_{t} / A_{e}=0.4$ and $P_{\infty}=8.5$.

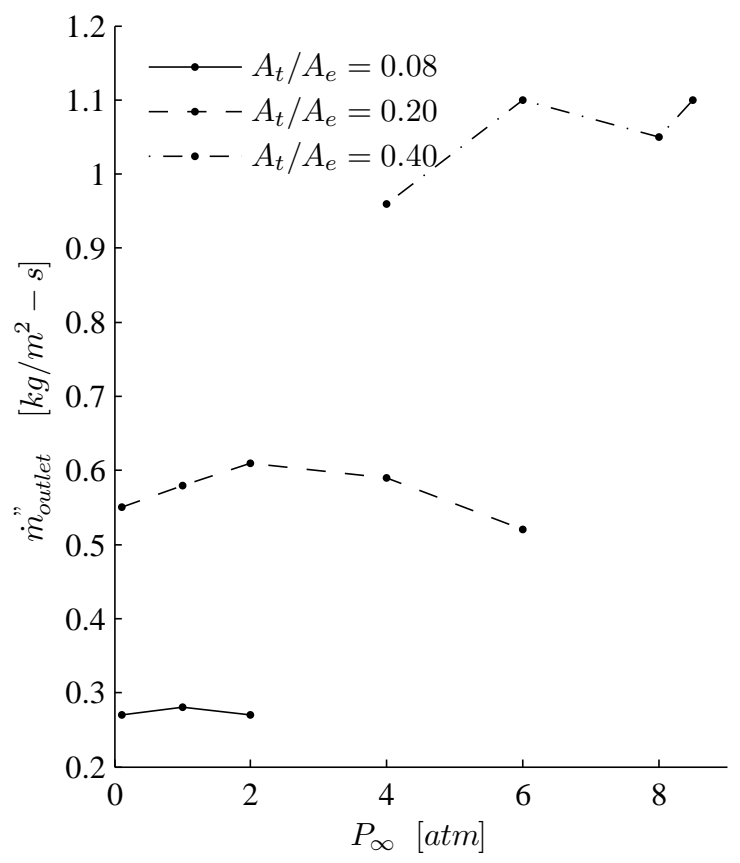

FIGURE 5.23: Prediction of mass flux at the outlet of the combustion chamber from 2D RDC simulation with $A_{t} / A_{e}=0.08,0.02$ and 0.4 at elevated pressures

The parameters predicted using the analytical model in Section 5.1 were also calculated based on the results of the 2D CFD simulations. The variation of $\Theta$ and $P_{o, l o s s}$ with the injection 
geometry and $P_{\infty}$ are initially presented by themselves and a comparison with the analytical prediction is presented afterwards.

The predicted variation of the losses in total pressure in RDC are presented in Figure 5.24. For a constant area ration $\left(A_{t} / A e\right)$, it is possible to relate the behavior of the pressure losses with the three operational regimes described previously. First, the losses in total pressure at low values of $P_{\infty}$ are initially low. At this regime the gases expand behind the detonation wave and only a portion of them are influenced by the oblique shock wave. The majority of the losses in total pressure under this conditions are caused by the injection section, where the nonisentropic expansion of the gases occur. This is evidenced by the fact that once the area ratio is increased the losses in total pressure for supersonic operation dramatically decrease. Moreover, as $P_{\infty}$ increases, the losses in total pressure initially rise. This is explained by the appearance of the shock wave normal to the flow that spans in the circumference of the combustion chamber. As the detonation products pass through such shock wave their entropy increases and total pressure decreases. The maxima of the total pressure loss is correlated to the conditions at which the strength of the shock wave is bigger. If $P_{\infty}$ further increases, the location of the shock wave moves upstream in the axial direction of the combustion chamber. Since the velocity of the gases closer to the inlet surface is lower, then the strength of the shock wave decreases and the losses in total pressure do as well. The regime at which the losses in total pressure reached their minimum value correspond to that of mostly subsonic detonation products.

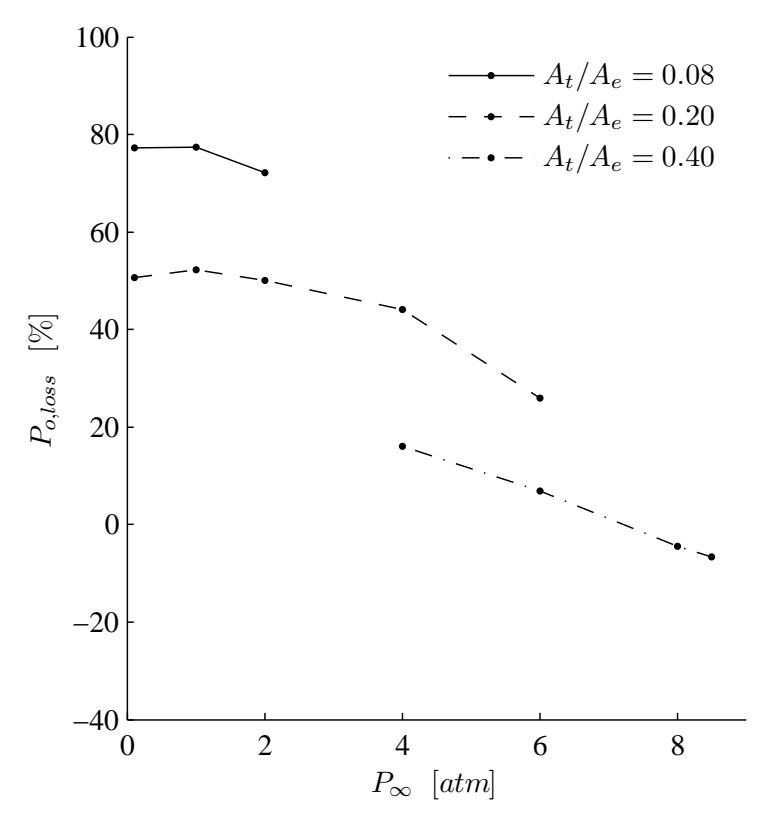

FIGURE 5.24: Prediction of total pressure losses at the outlet of the combustion chamber from 2D RDC simulation with $A_{t} / A_{e}=0.08,0.02$ and 0.4 at elevated pressures

Pressure gain combustion (PGC) was evidenced for two operating conditions in the present study, illustrated as negative values of the percentage in the losses of pressure in Figure 5.24. The CFD simulations for $A_{t} / A_{e}=0.4$ and $P=8.0$ and 8.5 atm led to a pressure gain 
of $4.4 \%$ and $6.7 \%$, respectively when calculated with respect to the manifold pressure $P_{o, m}$.

The non dimensional geometric factor $\Theta$ defined in Section 5.1, was also calculated for the 2D CFD simulations. As illustrated in Figure 5.25, $\Theta$ decreases as $P_{\infty}$ increases, and increases as $A_{t} / A_{e}$ increases. Additionally, it was found that the cutoff value for the possibility of continuous detonation is in average 0.14 .

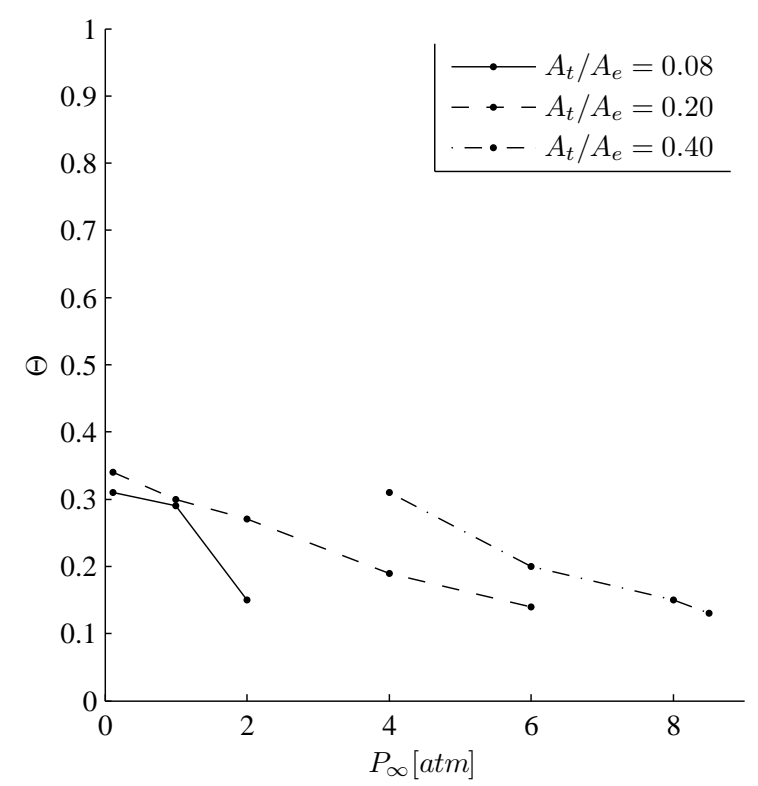

FIGURE 5.25: Prediction of geometric factor $\Theta$ from 2D RDC simulation with $A_{t} / A_{e}=$ $0.08,0.02$ and 0.4 at elevated pressures

The prediction of the losses in total pressure and the geometrical parameter $\Theta$ from the analytical model and the 2D CFD simulations are compared in Figure 5.26(a) and (b). The losses in total pressure are appropriately predicted for the lower values of $P \infty$ of each of the geometrical configurations. However as $P_{\infty}$ increases, the theoretical model underestimates the losses in total pressure leading to higher levels of PGC. Despite these differences, the analytical model shows the trends predicted using 2D CFD simulations. These results point to the fact that in order to obtain PGC in RDC elevated far field pressures and large $A_{t} / A_{e}$ ratios are required. In regards to the value of the non dimensional factor $\Theta$ it is observed that good agreement between the analytical and the CFD predictions were obtained with $P_{\infty}=2.0$ atm, $A_{t} / \mathrm{Ae}=0.08$ and 0.2 . Despite the fact there is an agreement in the trend of $\Theta$ between the analytical and the $2 \mathrm{D}$ CFD simulation, it is observed that the in general the values from the analytical model are substantially lower than those obtained from the CFD simulations. A further analysis showed that the differences were caused by the value of $\theta_{r}$, and that as shown in Figure 5.26(c), the predicted values of the detonation front height $\Delta_{D W}$ from the analytical and CFD calculations show good agreement, especially for elevated far field pressures.

The comparison between the analytical and the CFD predicts allows one to conclude that despite the large number of assumptions and simplifications performed in the analytical 


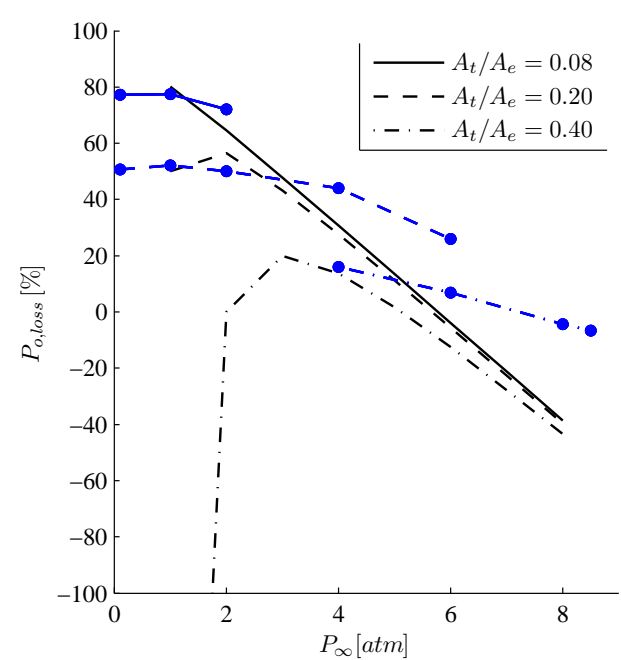

(a)

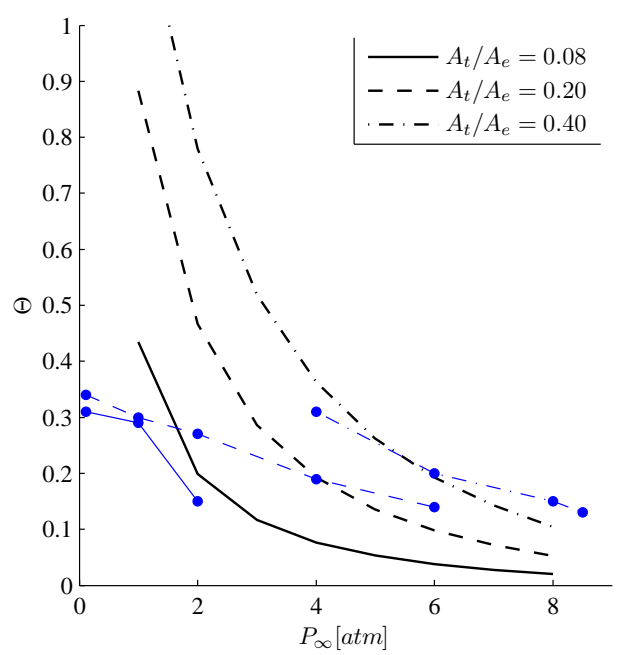

(b)

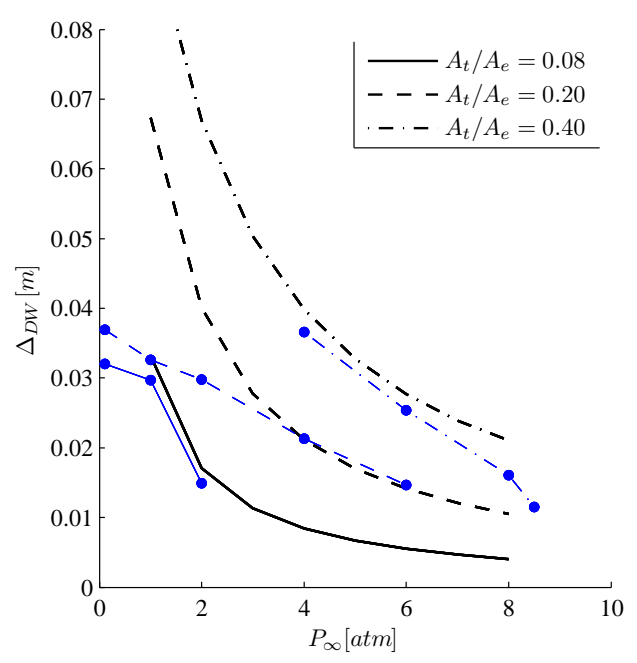

(c)

FIGURE 5.26: Comparison of predicted RDC performance parameters from analytical model and 2D CFD calculations. $P_{o, l o s s}(\mathrm{a}), \Theta(\mathrm{b})$ and $\Delta_{D W}(\mathrm{c})$

model, it has the capability of predicting the general trends of the performance parameters in RDC at elevated pressure operations.

A table summarizing the results obtained for the 2D CFD simulations of RDC in elevated far field pressure conditions is presented in Table 5.1

It is concluded that the developed analytical tool is capable of predicting the conditions viable for obtaining continuous detonation and PGC in RDC. The model developed in the present study, was proven capable of determining the governing trend of total pressure loss and detonation wave height for RDC at elevated far field pressures. In addition to the predictive nature of the model, it was proven critical to providing initial conditions leading to continuous detonation in RDC simulations at high values of $P_{\infty}$. 
TABLE 5.1: 2D RDC Results for elevated outlet pressures

\begin{tabular}{ccccccccc}
\hline \hline$A_{t} / A_{e}$ & $P_{\infty}$ & $\hat{P}_{\text {o,inlet }}$ & $P_{\text {o,loss }}$ & $\Delta_{D W}$ & $U_{D W}$ & $\theta_{r}$ & $\dot{m}_{\text {outlet }}$ & $\Theta$ \\
\hline- & atm & atm & - & $\mathrm{mm}$ & $\mathrm{m} / \mathrm{s}$ & $\mathrm{cm}$ & $\frac{\mathrm{kg}}{\mathrm{m}^{2}-s}$ & - \\
\hline 0.08 & 0.11 & 2.56 & $77.3 \%$ & 32 & 1863 & 21.97 & 0.27 & 0.31 \\
0.08 & 1 & 2.55 & $77.4 \%$ & 29.7 & 1893 & 22.15 & 0.28 & 0.29 \\
0.08 & 2 & 3.15 & $72.1 \%$ & 14.9 & 1941 & 22.46 & 0.27 & 0.15 \\
0.2 & 0.11 & 5.58 & $50.6 \%$ & 36.9 & 1892 & 20.82 & 0.55 & 0.34 \\
0.2 & 1 & 5.41 & $52.2 \%$ & 32.6 & 1893 & 20.58 & 0.58 & 0.30 \\
0.2 & 2 & 5.63 & $50.1 \%$ & 29.8 & 1893 & 20.38 & 0.61 & 0.27 \\
0.2 & 4 & 6.32 & $44.1 \%$ & 21.3 & 1938 & 20.23 & 0.59 & 0.19 \\
0.2 & 6 & 8.36 & $26.0 \%$ & 14.7 & 1955 & 21.51 & 0.52 & 0.14 \\
0.4 & 4 & 9.49 & $16.0 \%$ & 36.6 & 1977 & 19.15 & 0.96 & 0.31 \\
0.4 & 6 & 10.52 & $6.9 \%$ & 25.4 & 1897 & 17.25 & 1.1 & 0.20 \\
0.4 & 8 & 11.8 & $-4.4 \%$ & 16.1 & 1955 & 21.47 & 1.05 & 0.15 \\
0.4 & 8.5 & 12.06 & $-6.7 \%$ & 11.5 & 1954 & 24.84 & 1.1 & 0.13 \\
\hline
\end{tabular}

It can then be concluded based on the 2D CFD simulations that it is feasible to achieve pressure gain combustion in RDC if the operating and geometrical conditions are appropriately selected. For the geometric configuration considered in the present study, the maximum level of PGC obtained in the present was $6.7 \%$, corresponding to $P_{\infty}=8.5 \mathrm{~atm}$ and $A_{t} / A_{e}=0.4$.

\subsection{Conclusions from Rotating Detonation Combustion at Elevated Pressures}

- An analytical model for the prediction of temperature, pressure and species distribution in RDC was developed. The developed model was based on analytical formulation for the $1 \mathrm{D}$ distribution of the thermodynamic sate of the products in RDC and the ideal injection model. The developed formulation was implemented to prescribe the initial condition in 2D and 3D RDC simulations favorable to continuous detonation. Moreover, it was used as a predictive tool to estimate the conditions at which continuous detonation and PGC can be achieve in RDC.

- A strategy to appropriately select the parameters necessary for the implementation of NRBC in RDC was developed. The possibility of the solution to drift from the imposed boundary condition was demonstrated. It was also concluded that for the selected value of $\sigma=-2.1 \pi$, the NRBC controls the pressure in the mean flow and reduces the reflection of the pressure disturbances from the outlet boundary.

- The assessment of the performance of NRBC for RDC simulations presented as part of the current research is, to the best of the author's knowledge, the first to be reported. 
- The effects of injection geometry and far field pressure on RDC performance was evaluated using 2D CFD simulations. Three throat to expansion ratios $\left(A_{t} / A_{e}\right)$ equal to 0.08 , 0.2 and 0.4 were evaluated. Continuous detonation combustion was predicted for far field pressures of $0.1,1.0,2.0,4.0,6.0,8.0$ and $8.5 \mathrm{~atm}$.

- Three distinct flow regimes were identified as a function of the flow structures present inside the combustion chamber. The role of increased far field pressure and $A_{t} / A_{e}$ on the definition of such regimes was addressed.

- The onset of flow instabilities in RDC was evidenced for flow conditions where the majority of the detonation products operate at subsonic velocities.

- The governing factors contributing to the increase of losses in total pressure were identified. It was concluded that the losses in total pressure increase significantly when $A_{t} / A_{e}$ decreases. Additionally, it was identified that the presence of a strong shock wave covering the circumference of the combustion chamber is a major contributor to the losses in total pressure.

- The non-dimensional geometric parametr $\Theta$ was defined in order to characterized the predicted detoantion height and the deflagration front shape. Such parameter was used to establish the viability of obtaining continuous detonation under different operating conditions and injection geometries.

- The geometric characteristics of the deflagration and detonation front in RDC for elevated far field pressure conditions were calculated. It was concluded that for the studied set up, continuous detonation cannot be sustained if $\Theta$ is smaller than 0.13 .

- The losses in total pressure were calculated from the 2D CFD simulations. It was concluded that PGC in RDC can be achieved for high values of $P_{\infty}$ and large values of $A_{t} / A_{e}$. For the configuration studied in the current study, it was observed that a gain in total pressure of $4.4 \%$ and $6.7 \%$ is obtained with $A_{t} / A_{e}=0.4$

- It is concluded that the developed computational fluid dynamic modeling strategy maintained generality, low computational cost, and the capability to accurately predict RDC under operation conditions relevant to gas turbine.

- Adequate configurations and operating conditions for PGC in RDC were identified; this was achieved through a parametric study were the developed modeling strategy was implemented. 


\section{Chapter 6}

\section{General Conclusions}

Detailed conclusions for the different sections of the present study are presented at the end of each chapter. However, a summary of the most relevant conclusions are presented as follows:

Numerical simulations of the non reactive shock tube problem were performed using the DBS and PBS in the commercial CFD solver ANSYS-Fluent. They adequately captured the flow discontinuities present in compressible flows. It was concluded that under the considered conditions, using a computational cell size $\delta_{x}<4 m m$ leads to relative error of the shock wave velocity lower than $1 \%$. Based on the results of such simulations, the numerical models selected for the present study were: DBS-AUSM-SQC-2UP, and PBS-PISO-SQC-2UP. It was also concluded that despite implementing second order spatial discretization scheme (2UP), first order convergence was obtained.

Based on the CFD simulations for the propagation of a planar detonation wave in a 2D channel, it was concluded that the detonation wave velocity and the general behavior of the detonation wave structures are appropriately predicted. Relative errors calculated with respect to the CJ conditions show that for $\delta x=0.1 \mathrm{~mm}$ the predicted values do not differ with the theoretical solution for more than $1 \%$. It was also concluded that numerical instabilities arise in the 2D simulation of a detonation wave if the CFL number surpassed 2.0. It was identified that the behavior of these numerical instabilities leads to non physical cellular structures behind the detonation front.

Based on the two dimensional simulations of RDC for the selected validation case, it was showen that adequate prediction of the flow structures in RDC are obtained. The relative error of the predicted detonation wave velocity with respect to the experimental value while using the 1 step and multi step reaction mechanism were equal to $7.0 \%$ and $5.0 \%$, respectively. It was also concluded that the detonation wave velocity consistently decreases when $3 \mathrm{D}$ effects 
are considered. In fact, the relative error for the single step and multi step reaction mechanism were equal to $6 \%$ and $1 \%$ respectively.

The emission characteristics for RDC were determined. It was concluded that for stoichiometric $\mathrm{H}_{2}$-air mixture, a time averaged concentration of dried $\mathrm{NO}$ corrected for $15 \% \mathrm{O}_{2}$ equal to $164 \mathrm{ppm}$ was predicted. However, under fuel lean conditions $(\Phi=0.5)$, the predicted concentration of dried $\mathrm{NO}$ corrected for $15 \% \mathrm{O}_{2}$ was equal to $35 \mathrm{ppm}$, a reduction of $79 \%$.

The effects of the curvature of the combustion chamber were studied through 3D inviscid simulations of RDC under the validation conditions. It was concluded that strong pressure gradients in the radial direction are formed behind the detonation front. Additionally, it was obtained that the pressure gradient in the radial direction is approximately equal to $400 \mathrm{~atm} / \mathrm{m}$. In addition, the effects of heat transfer at the wall in 3D simulations of RDC were analyzed. It was obtained the $0.3 \%$ and $1.3 \%$ of the influx of energy is dissipated through the wall, depending of the wall boundary condition implemented.

As part of the current study, a 2D analytical model for the prediction of temperature, pressure and species distribution in RDC was developed. The developed formulation was implemented to prescribe the initial condition in 2D and 3D RDC simulations favorable to continuous detonation, allowing one to reduce the computational time and expanding the range of $P_{\infty}$ at which continuous detonation could be initialized and sustained. It was also concluded that the developed analytical model is a useful predictive tool to estimate the conditions at which continuous detonation and PGC can be achieve in RDC. It was observed that it appropriately predicts the influence on the losses in total pressure and the detonation front height due to changes in operating conditions and injection geometry.

In the present study a strategy to determine the appropriate parameters for the implementation of the NRBC and the LRM on the simulation of RDC at elevated outlet pressures was developed, and the influence of the relaxation parameter $\sigma$ was determined. It was concluded that using the frequency of the detonation wave as the cutoff frequency for the calculation of $\sigma$, the reflection of the pressure waves is significantly reduced and the mean pressure at the outlet is adequately controlled.

The influence of increasing the far field pressure on the flow characteristics of RDC was studied. It was concluded that the flow inside the combustion chamber can be classified into three distinct regimes as the far field pressure is increased: supersonic regime, mixed regime and mostly subsonic regime. The performance characteristics and flow structures of each regimes were described. It was observed that the supersonic regime can only be obtained at low outlet pressure. Under such conditions very little total pressure losses occur during the combustion regime, however big losses occur during the injection process. On the other hand, for mixed regime operation, shock waves in the circumferential direction of the combustion chamber are 
formed and large losses in the total pressure are experienced. The mostly subsonic regime presents the lowest losses in total pressure, even leading to PGC; however, it was observed that under such conditions the flow becomes highly unstable.

It was also concluded that in order to obtain pressure gain combustion in RDC, the losses in total pressure from the injection process need to be minimized. Based on the simple converging-diverging injection geometry, it was obtained that increasing the throat to expansion ratio $\left(A_{t} / A_{e}\right)$ significantly reduces the losses in total pressure. In addition it was concluded that the losses in total pressure are reduced as $P_{\infty}$ increases. Increasing $P_{\infty}$ reduces the strength, and even eliminates the presence of the circumferential shock wave, the main contributing structure to the losses in total pressure.

\subsection{Recommendation for Future Work}

It is consider by the author that the computational strategy developed in the current study can serve as foundation for future research on RDC at operating conditions relevant for stationary power generation. The fact that the present study was performed using a widely adopted commercial CFD solver (ANSYS-Fluent) is not to be undermined. By establishing the general guidelines necessary for the prediction of the main governing parameters of RDC at elevated outlet pressures, it is intended to contribute to the scientific and engineering community a mean to perform research on RDC in more complex geometries and under different operating conditions.

In addition to the adaptation and optimization of the combustion chamber and injection geometry, it is consider by the author that the integration of the compression stage and turbine stage with the combustion chamber for RDC is to be address. As part of the research project in which the current research was performed, initial simulations on the integration of the stator stage with the combustion chamber were achieved. It was observed that significant changes on the flow structures inside the combustion chamber occur. The problem of the integration of an RDC combustion chamber with the downstream and upstream components of the stationary power GT creates is a very complex challenge. It is believed that CFD will play a very important role in understanding the most efficient way to overcome such technical difficulties. 


\section{Appendix A}

\section{Chemical Model and Reaction Mechanisms}

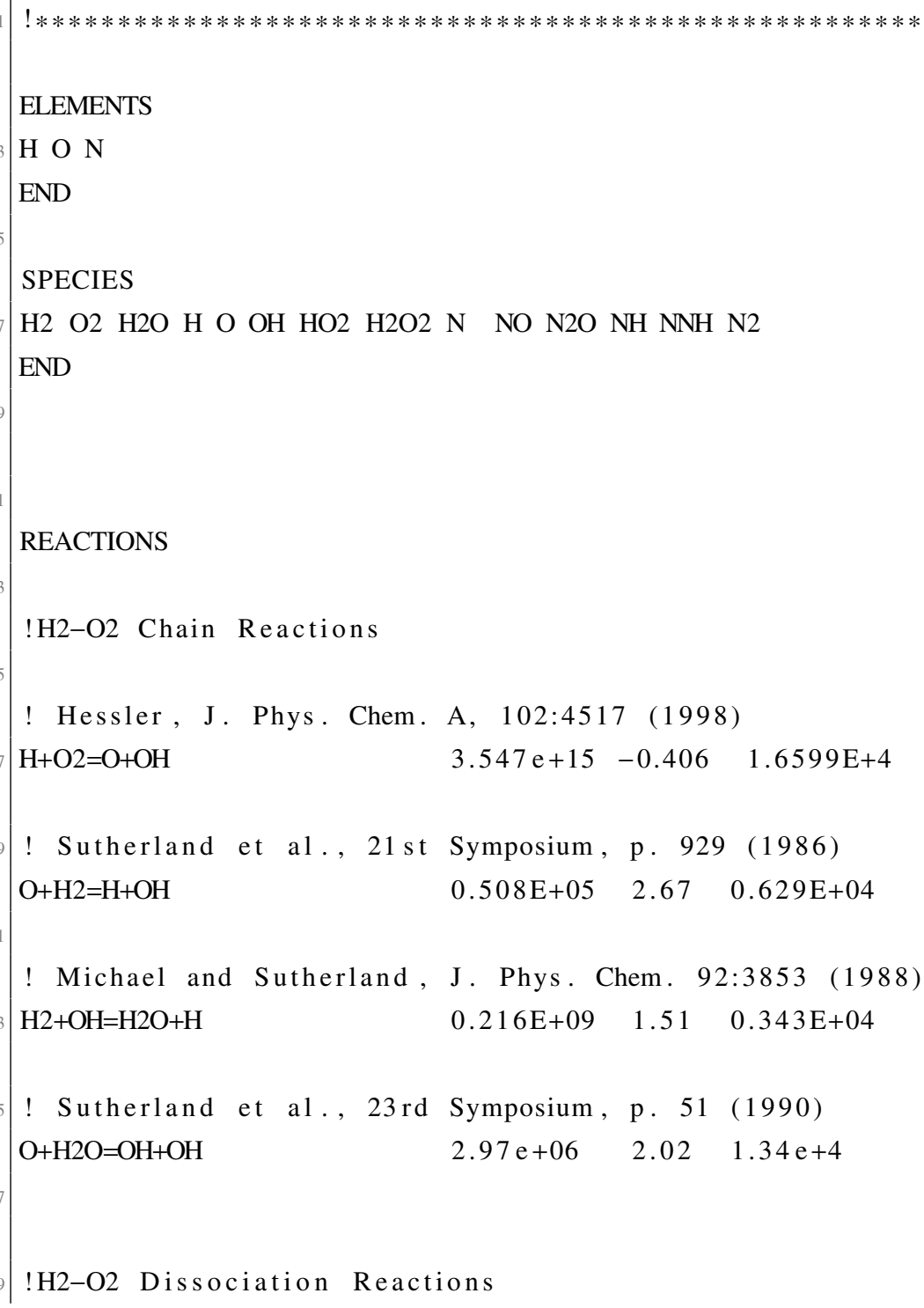




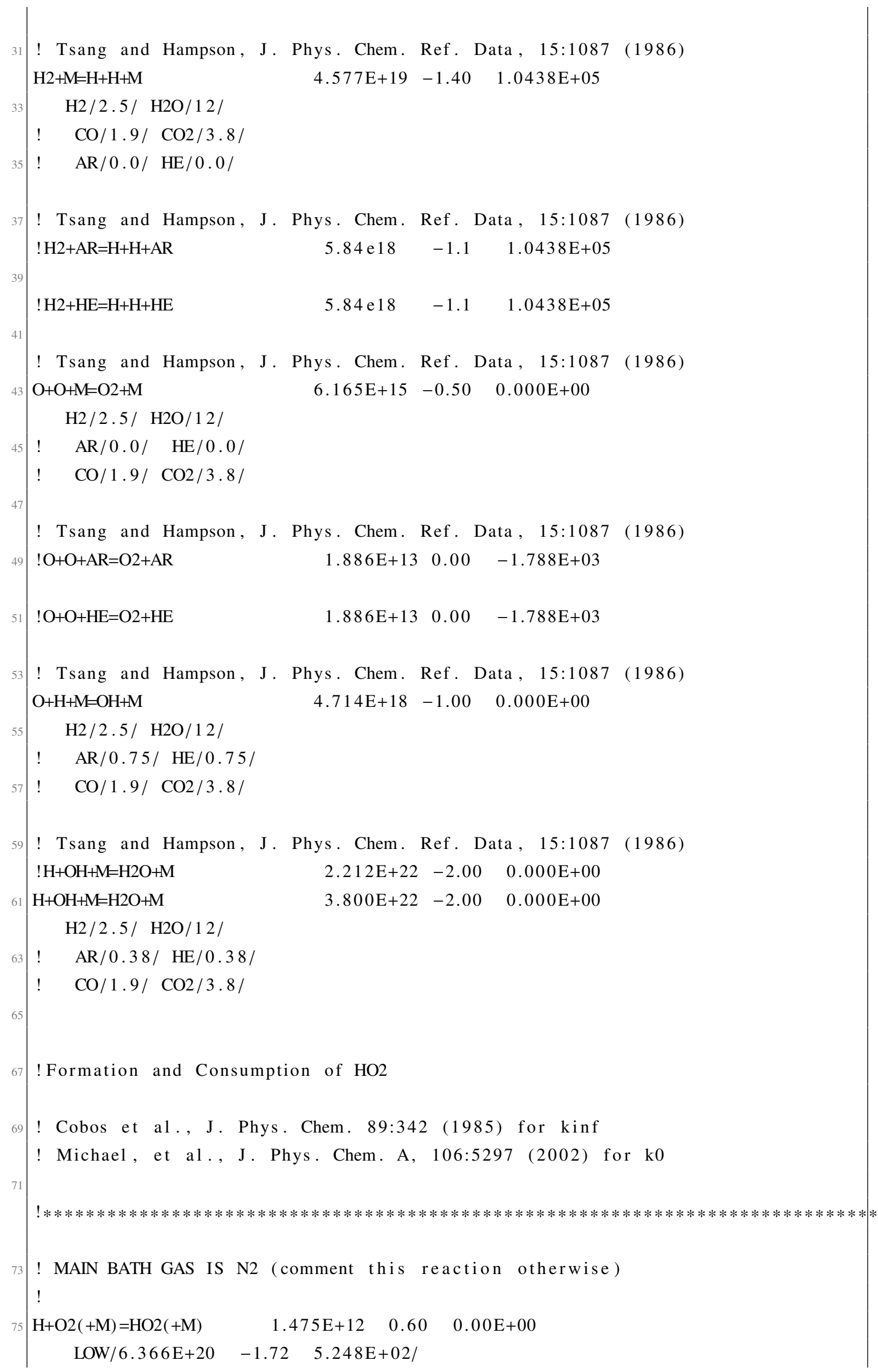




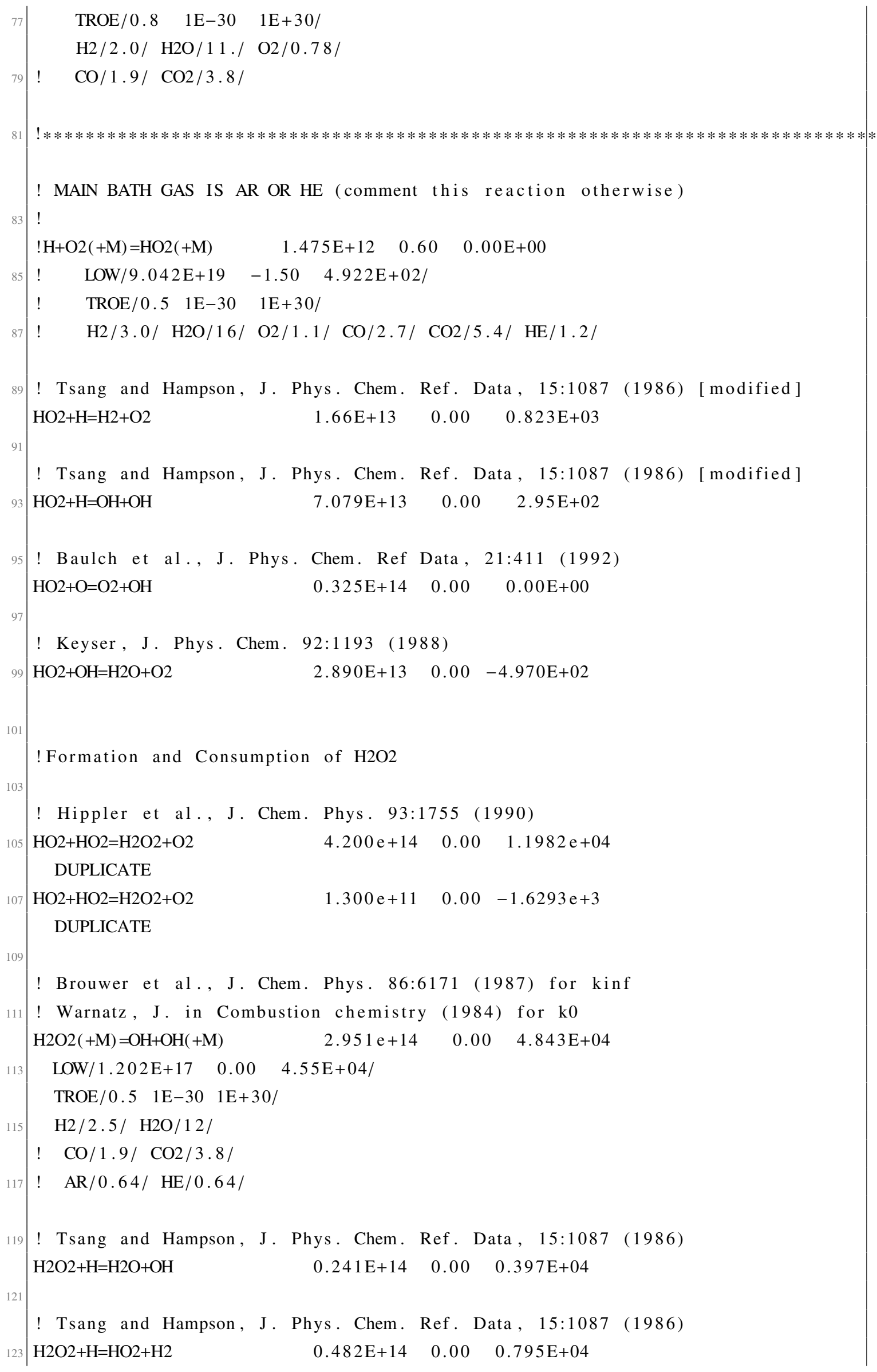




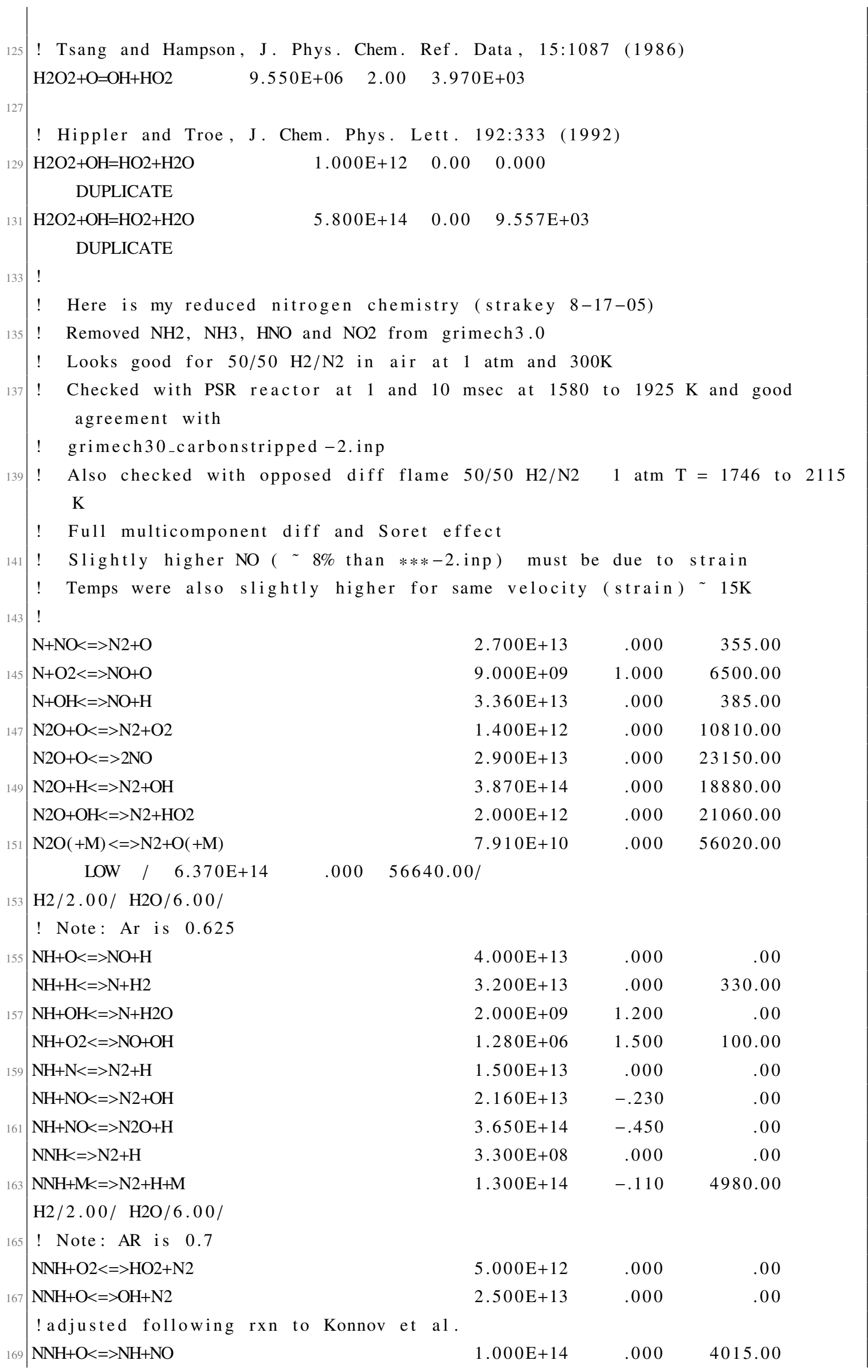




\begin{tabular}{llll|}
171 & $5.000 \mathrm{E}+13$ & .000 & .00 \\
$\mathrm{NNH}+\mathrm{H}<=>\mathrm{H} 2+\mathrm{N} 2$ & $2.000 \mathrm{E}+13$ & .000 & .00 \\
$\mathrm{NNH}+\mathrm{OH}<=>\mathrm{H} 2 \mathrm{O}+\mathrm{N} 2$ & & & \\
\hline & & & \\
$\mathrm{END}$ & & & \\
\hline
\end{tabular}

Appendices/chem.inp 


\section{Appendix B}

\section{Inlet Boundary Condition - UDF}

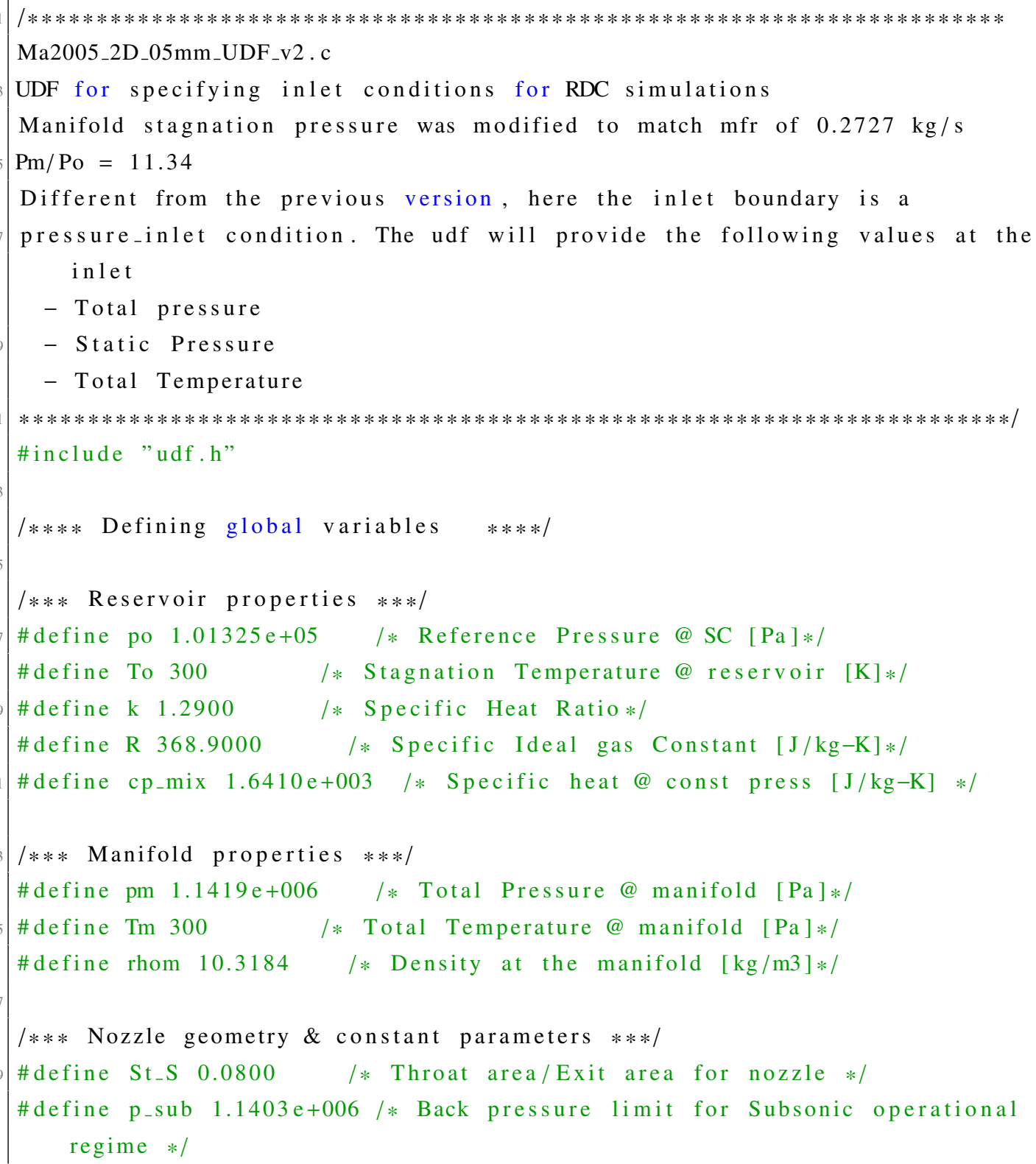




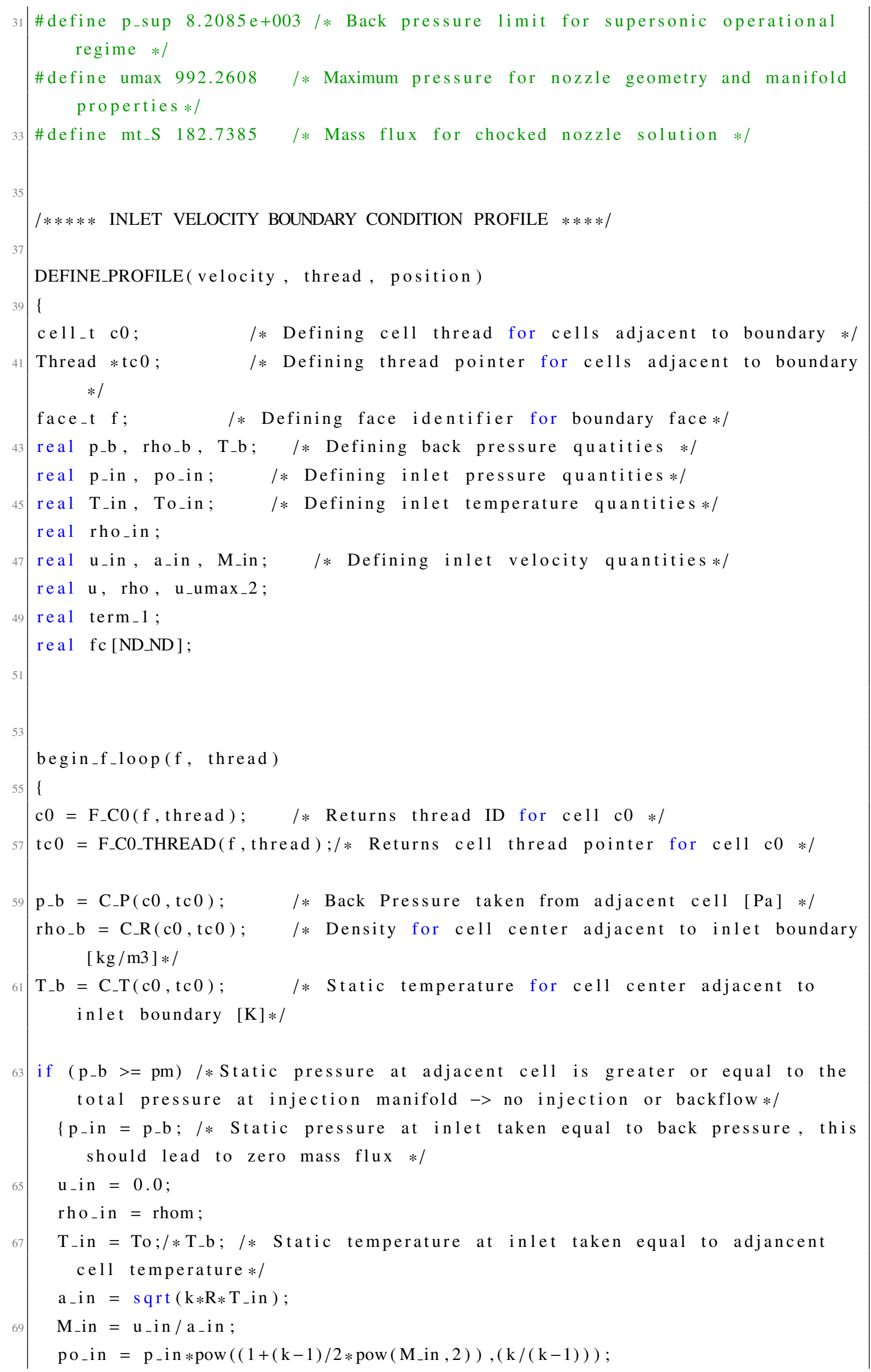


To_in $=\mathrm{T}_{\text {in }} *\left(1+(\mathrm{k}-1) / 2 *\right.$ pow $\left.\left(\mathrm{M}_{\_} \mathrm{in}, 2\right)\right)$;

F_PROFILE (f, thread, position $)=u_{-}$in ;

\}

else

\{

if $\left(p_{-} b>=p_{-} s u b\right)$

$\left\{p_{-}\right.$in $=p_{-} b$;

$\mathrm{u}_{-} \mathrm{in}=\operatorname{umax} * \mathrm{sqrt}\left(\operatorname{pow}\left(1-\left(\mathrm{p}_{-} \mathrm{in} / \mathrm{pm}\right),((\mathrm{k}-1) / \mathrm{k})\right)\right)$;

rho_in $=\operatorname{rhom} * \operatorname{pow}\left(\left(1-\operatorname{pow}\left(\left(\mathrm{u}_{-}\right.\right.\right.\right.$in $/$umax $\left.\left.\left.), 2\right)\right),(1 /(\mathrm{k}-1))\right)$;

$\mathrm{T}_{-}$in $=\mathrm{p}_{-} \mathrm{in} / \mathrm{rho}_{-} \mathrm{in} / \mathrm{R}$;

$\mathrm{a}_{-} \mathrm{in}_{\mathrm{in}}=\operatorname{sqrt}\left(\mathrm{k} * \mathrm{R} * \mathrm{~T}_{-}\right.$in $)$;

$M_{-}$in $=u_{-}$in $/ a_{-}$in ;

po_in $=p_{-}$in $*$ pow $\left(\left(1+(\mathrm{k}-1) / 2 * \operatorname{pow}\left(M_{-}\right.\right.\right.$in, 2$\left.\left.)\right),(\mathrm{k} /(\mathrm{k}-1))\right)$;

To_in $=\mathrm{T}_{-}$in $*\left(1+(\mathrm{k}-1) / 2 *\right.$ pow $\left(M_{-}\right.$in, 2$\left.)\right)$;

F_PROFILE ( f , thread, position $)=u_{-}$in ;

\}

else

\{

if $\left(p_{-} b>=p_{-} s u p\right)$

$\left\{\mathrm{p}_{-} \mathrm{in}=\mathrm{p}_{-} \mathrm{b}\right.$;

term_ $1=(2 * \mathrm{k} /(\mathrm{k}-1)) *\left(\mathrm{p}_{-} \mathrm{in} / \mathrm{mt}_{-} \mathrm{S}\right)$;

$\mathrm{u}_{-}$in $=-0.5 * \operatorname{term} \mathrm{t}_{-} 1+0.5 * \operatorname{sqrt}\left(\operatorname{pow}\left(\operatorname{term}_{-} 1,2\right)+4 * \operatorname{pow}\left(\mathrm{umax}_{1}, 2\right)\right)$;

rho_in $=\mathrm{mt}_{-} \mathrm{S} / \mathrm{u}_{-} \mathrm{in}$;

$\mathrm{T}_{-}$in $=\mathrm{p}_{-}$in $/ \mathrm{rho}_{-} \mathrm{in} / \mathrm{R}$;

$\mathrm{a}_{-}$in $=\operatorname{sqrt}\left(\mathrm{k} * \mathrm{R} * \mathrm{~T}_{-}\right.$in $)$;

M_in $=u_{-}$in $/ a_{-}$in ;

po_in $=p_{-}$in $*$ pow $\left(\left(1+(\mathrm{k}-1) / 2 * \operatorname{pow}\left(M_{-}\right.\right.\right.$in, 2$\left.\left.)\right),(\mathrm{k} /(\mathrm{k}-1))\right)$;

To_in $=\mathrm{T}_{-}$in $*\left(1+(\mathrm{k}-1) / 2 *\right.$ pow $\left(\mathrm{M}_{-}\right.$in, 2$\left.)\right)$;

F_PROFILE ( f, thread, position ) = u_in ;

\}

els e

$\left\{\mathrm{p}_{-}\right.$in $=\mathrm{p}_{-}$sup

term_ $1=(2 * \mathrm{k} /(\mathrm{k}-1)) *\left(\mathrm{p}_{-}\right.$in $\left./ \mathrm{mt}_{-} \mathrm{S}\right)$;

$\mathrm{u}_{-}$in $=-0.5 * \operatorname{term} \mathrm{t}_{-} 1+0.5 * \operatorname{sqrt}\left(\operatorname{pow}\left(\operatorname{term}_{-} 1,2\right)+4 * \operatorname{pow}\left(\operatorname{umax}_{2}, 2\right)\right)$;

rho_in $=$ mt_s/u_in;

$\mathrm{T}_{-}$in $=\mathrm{p}_{-}$in $/ \mathrm{rho}_{-} \mathrm{in} / \mathrm{R}$;

$\mathrm{a}_{-}$in $=\operatorname{sqrt}\left(\mathrm{k} * \mathrm{R} * \mathrm{~T}_{-}\right.$in $)$;

$M_{-}$in $=u_{-}$in $/ a_{-}$in ;

po_in $=p_{-}$in $* \operatorname{pow}\left(\left(1+(\mathrm{k}-1) / 2 * \operatorname{pow}\left(M_{-}\right.\right.\right.$in, 2$\left.\left.)\right),(\mathrm{k} /(\mathrm{k}-1))\right)$;

To_in $=\mathrm{T}_{-}$in $*\left(1+(\mathrm{k}-1) / 2 *\right.$ pow $\left(\mathrm{M}_{-}\right.$in, 2$\left.)\right)$;

F_PROFILE ( f, thread, position $)=u_{-}$in ;

\}

\}

\}

$15\}$

end_f_loop (f, thread) 
Appendices/Ma2005_2D_05mm_UDF_vel_inlet.c 


\section{Appendix C}

\section{Numerical Simulations of}

\section{Non-Reactive Shock Tube Problem}

TABLE C.1: Numerical Simulations for Non Reactive Shocktube using DBS and Mesh-1

\begin{tabular}{|c|c|c|c|c|c|c|}
\hline \hline Case Name & Solver & Flux Type & Gradient & Discr. Scheme & $U_{S W}\left[\frac{m}{s}\right]$ & $\% E U_{S W}$ \\
\hline M1-DBS-ROE-GC-1U & DBS & ROE & GC & 1 1UP & 605.71 & 6.4 \\
M1-DBS-ROE-GC-2U & DBS & ROE & GC & $2 U P$ & 582.86 & 2.4 \\
M1-DBS-ROE-GC-3M & DBS & ROE & GC & $3 \mathrm{M}$ & 582.86 & 2.4 \\
\hline M1-DBS-ROE-SQC-1U & DBS & ROE & SQC & $1 U P$ & 605.71 & 6.4 \\
M1-DBS-ROE-SQC-2U & DBS & ROE & SQC & 2UP & 582.86 & 2.4 \\
M1-DBS-ROE-SQC-3M & DBS & ROE & SQC & 3M & 582.86 & 2.4 \\
\hline M1-DBS-AUSM-GC-1U & DBS & AUSM & GC & 1UP & 582.86 & 2.4 \\
M1-DBS-AUSM-GC-2U & DBS & AUSM & GC & 2UP & 582.86 & 2.4 \\
M1-DBS-AUSM-GC-3M & DBS & AUSM & GC & 3M & 582.86 & 2.4 \\
\hline M1-DBS-AUSM-SQC-1U & DBS & AUSM & SQC & 1UP & 605.71 & 6.4 \\
M1-DBS-AUSM-SQC-2U & DBS & AUSM & SQC & 2UP & 582.86 & 2.4 \\
M1-DBS-AUSM-SQC-3M & DBS & AUSM & SQC & 3M & 582.86 & 2.4 \\
\hline
\end{tabular}

TABLE C.2: Influence of mesh cell size on numerical Simulations for Non Reactive Shocktube.

Calculated using DBS-AUSM-SQC-2U

\begin{tabular}{|c|c|c|c|c|c|c|}
\hline \hline Case Name & Solver & Flux Type & Gradient & Discr. Scheme & $U_{S W}\left[\frac{m}{s}\right]$ & $\% E U_{S W}$ \\
\hline M1-DBS-AUSM-SQC-2U & DBS & AUSM & SQC & 2UP & 582.86 & 2.4 \\
M2-DBS-AUSM-SQC-2U & DBS & AUSM & SQC & 2UP & 571.43 & 0.4 \\
M3-DBS-AUSM-SQC-2U & DBS & AUSM & SQC & 2UP & 569.14 & $<0.1$ \\
M4-DBS-AUSM-SQC-2U & DBS & AUSM & SQC & 2UP & 570.29 & 0.2 \\
\hline
\end{tabular}


TABLE C.3: Numerical Simulations for Non Reactive Shocktube using PBS and Mesh-1

\begin{tabular}{|c|c|c|c|c|c|c|}
\hline \hline Case Name & Solver & Flux Type & Gradient & Discr. Scheme & $U_{S W}\left[\frac{m}{s}\right]$ & $\% E U_{S W}$ \\
\hline M1-PBS-PISO-GC-1U & PBS & PISO & GC & 1 UP & 582.86 & 2.4 \\
M1-PBS-PISO-GC-2U & PBS & PISO & GC & 2UP & 582.86 & 2.4 \\
M1-PBS-PISO-GC-Q & PBS & PISO & GC & QUICK & 582.86 & 2.4 \\
M1-PBS-PISO-GC-3M & PBS & PISO & GC & $3 \mathrm{M}$ & 582.86 & 2.4 \\
\hline M1-PBS-PISO-SQC-1U & PBS & PISO & SQC & 1 UP & 582.86 & 2.4 \\
M1-PBS-PISO-SQC-2U & PBS & PISO & SQC & 2UP & 582.86 & 2.4 \\
M1-PBS-PISO-SQC-Q & PBS & PISO & SQC & QUICK & 582.86 & 2.4 \\
M1-PBS-PISO-SQC-3M & PBS & PISO & SQC & $3 \mathrm{M}$ & 582.86 & 2.4 \\
\hline M1-PBS-CP-GC-1U & PBS & COUPLED & SQC & 1 UP & 582.86 & 2.4 \\
M1-PBS-CP-GC-2U & PBS & COUPLED & SQC & 2 UP & 582.86 & 2.4 \\
M1-PBS-CP-GC-Q & PBS & COUPLED & SQC & QUICK & 582.86 & 2.4 \\
M1-PBS-CP-GC-3M & PBS & COUPLED & SQC & $3 \mathrm{M}$ & 582.86 & 2.4 \\
\hline M1-PBS-CP-SQC-1U & PBS & COUPLED & SQC & 1UP & 582.86 & 2.4 \\
M1-PBS-CP-SQC-2U & PBS & COUPLED & SQC & 2UP & 582.86 & 2.4 \\
M1-PBS-CP-SQC-Q & PBS & COUPLED & SQC & QUICK & 582.86 & 2.4 \\
M1-PBS-CP-SQC-3M & PBS & COUPLED & SQC & $3 \mathrm{M}$ & 582.86 & 2.4 \\
\hline
\end{tabular}

TABLE C.4: Influence of mesh cell size on numerical Simulations for Non Reactive Shocktube.

Calculated using PBS-CP-SQC-2U

\begin{tabular}{|c|c|c|c|c|c|c|}
\hline \hline Case Name & Solver & Flux Type & Gradient & Discr. Scheme & $U_{S W}\left[\frac{m}{s}\right]$ & $\% E U_{S W}$ \\
\hline M1-PBS-CP-SQC-2U & PBS & COUPLED & SQC & 2UP & 582.86 & 2.4 \\
M2-PBS-CP-SQC-2U & PBS & COUPLED & SQC & 2UP & 571.43 & 0.4 \\
M3-PBS-CP-SQC-2U & PBS & COUPLED & SQC & 2UP & 569.14 & $<0.1$ \\
M4-PBS-CP-SQC-2U & PBS & COUPLED & SQC & 2UP & 570.29 & 0.2 \\
\hline
\end{tabular}




\section{Bibliography}

Akbari, P., Nalim, R., and Mueller, N. (2006). A review of wave rotor technology and its applications. Journal of engineering for gas turbines and power, 128(4):717-735.

Bogey, C. and Bailly, C. (2002). Three-dimensional non-reflective boundary conditions for acoustic simulations: far field formulation and validation test cases. Acta Acustica united with Acustica, 88(4):463-471.

Bykovskii, F. and Vedernikov, E. (2003). Continuous detonation of a subsonic flow of a propellant. Combustion, Explosion and Shock Waves, 39(3):323-334.

Bykovskii, F. and Vedernikov, E. (2008). Continuous spin detonation of hydrogen-oxygen mixtures. 3. methods of measuring flow parameters and flow structure in combustors of different geometries. Combustion, Explosion, and Shock Waves, 44(4):451-460.

Bykovskii, F. and Vedernikov, E. (2009). Heat fluxes to combustor walls during continuous spin detonation of fuel-air mixtures. Combustion, Explosion, and Shock Waves, 45(1):70-77.

Bykovskii, F., Zhdan, S., and Vedernikov, E. (2009). Realization and modeling of continuous spin detonation of a hydrogen-oxygen mixture in flow-type combustors. 2. combustors with expansion of the annular channel. Combustion, Explosion, and Shock Waves, 45(6):716-728.

Bykovskii, F. A. and Mitrofanov, V. (1980). Detonation combustion of a gas mixture in a cylindrical chamber. Combustion, Explosion, and Shock Waves, 16(5):570-578.

Celik, I. (2007). Introductory Numerical Methods for Engineering Applications. Ararat Books \& Pub.

Chase, M. W. and Force, J. A. N. A. (1998). Nist-janaf thermochemical tables.

Design, R. (2013). Chemkin 10131.

Dou, H.-S., Tsai, H. M., Khoo, B. C., and Qiu, J. (2008). Simulations of detonation wave propagation in rectangular ducts using a three-dimensional weno scheme. Combustion and Flame, 154(4):644-659.

EIA (2013). Short-term energy outlook [electronic resource]. 
Escobar, S., Pakalapati, S. R., Celik, I., Ferguson, D., and Strakey, P. (2013). Numerical investigation of rotating detonation combustion in annular chambers. In ASME Turbo Expo 2013: Turbine Technical Conference and Exposition, pages V01AT04A071-V01AT04A071. American Society of Mechanical Engineers.

Ferguson, D. (2014). personal communication.

Ferziger, J. H. and Perić, M. (2002). Computational methods for fluid dynamics, volume 3. Springer Berlin.

Fluent, A. (2009). 12.0 theory guide. Ansys Inc, 5.

Frolov, S., Dubrovskii, A., and Ivanov, V. (2013). Three-dimensional numerical simulation of the operation of a rotating-detonation chamber with separate supply of fuel and oxidizer. Russian Journal of Physical Chemistry B, 7(1):35-43.

Fujiwara, T. and Tsuge, S.-i. (1972). Quasi-onedimensional analysis of gaseous free detonations. Journal of the Physical Society of Japan, 33(1):237-241.

Gemmen, R., Richards, G., and Janus, M. (1995). Pressure-gain combustion for gas turbines. Technical report, USDOE Morgantown Energy Technology Center, WV (United States).

Goodwin, D. (2005). Cantera: Object-oriented software for reacting flows.

Gordon, S. and McBride, B. J. (1972). Computer program for calculation of complex chemical equilibrium compositions.

Hans, H. (1934). Compound gas turbine and method of producing power therewith. US Patent $1,982,664$.

Heiser, W. H. and Pratt, D. T. (2002). Thermodynamic cycle analysis of pulse detonation engines. Journal of Propulsion and Power, 18(1):68-76.

Hirschfelder, J. O., Curtiss, C. F., Bird, R. B., et al. (1954). Molecular theory of gases and liquids, volume 26. Wiley New York.

Hishida, M., Fujiwara, T., and Wolanski, P. (2009). Fundamentals of rotating detonations. Shock Waves, 19(1):1-10.

Ingram, D., Jiang, B., and Causon, D. (1998). On the role of turbulence in detonation induced by mach stem reflection. Shock waves, 8(6):327-336.

Kindracki, J., Kobiera, A., Wolański, P., Gut, Z., Folusiak, M., and Swiderski, K. (2011a). Experimental and numerical study of the rotating detonation engine in hydrogen-air mixtures. In Progress in Propulsion Physics, volume 2, pages 555-582. EDP Sciences. 
Kindracki, J., Wolański, P., and Gut, Z. (2011b). Experimental research on the rotating detonation in gaseous fuels-oxygen mixtures. Shock Waves, 21(2):75-84.

Kuo, K. K. (1986). Principles of combustion. Wiley New York et al.

Launder, B. E. and Spalding, D. (1974). The numerical computation of turbulent flows. Computer methods in applied mechanics and engineering, 3(2):269-289.

Law, C. K. (2006). Combustion physics. Cambridge University Press.

Lee, J. H. (2008). The detonation phenomenon, volume 2. Cambridge University Press Cambridge.

Li, J., Zhao, Z., Kazakov, A., and Dryer, F. L. (2004). An updated comprehensive kinetic model of hydrogen combustion. International Journal of Chemical Kinetics, 36(10):566-575.

Lim, W. H. E. (2010). Gasdynamic inlet isolation in rotating detonation engine. PhD thesis, Monterey, California. Naval Postgraduate School.

Liu, S.-J., Lin, Z.-Y., Liu, W.-D., Lin, W., and Sun, M.-B. (2012). Experimental and threedimensional numerical investigations on h2/air continuous rotating detonation wave. Proceedings of the Institution of Mechanical Engineers, Part G: Journal of Aerospace Engineering, page 0954410011433542.

Ma, F., Choi, J.-Y., and Yang, V. (2005). Thrust chamber dynamics and propulsive performance of single-tube pulse detonation engines. Journal of propulsion and power, 21(3):512-526.

Ng, H., Radulescu, M., Higgins, A., Nikiforakis, N., and Lee, J. (2005). Numerical investigation of the instability for one-dimensional chapman-jouguet detonations with chain-branching kinetics. Combustion Theory and Modelling, 9(3):385-401.

Nicholls, J. A. and Cullen, R. E. (1964). The feasibility of a rotating detonation wave rocket motor. Technical report, DTIC Document.

Nordeen, C. A., Schwer, D., Schauer, F., Hoke, J., Cetegen, B., and Barber, T. (2011). Thermodynamic modeling of a rotating detonation engine. In 49th AIAA Aerospace Sciences Meeting, Orlando, FL.

Poinsot, T. and Veynante, D. (2011). Theoretical and Numerical Combustion, volume 3. CERFACS.

Radulescu, M. I., Sharpe, G. J., Law, C. K., and Lee, J. H. (2007). The hydrodynamic structure of unstable cellular detonations. Journal of Fluid Mechanics, 580:31-81.

Richards, G., Yip, J., Gemmen, R., Janus, M., Norton, T., and Rogers, W. (1993). Pressure-gain combustion. Technical report, USDOE Morgantown Energy Technology Center, WV (United States). 
Rudy, D. H. and Strikwerda, J. C. (1980). A nonreflecting outflow boundary condition for subsonic navier-stokes calculations. Journal of Computational Physics, 36(1):55-70.

Russo, R. M., King, P., Schauer, F., and Thomas, L. (2011). Characterization of pressure rise across a continuous detonation engine. In Joint Propulsion Conference.

Saravanamuttoo, H. I. H., Rogers, G. F. C., and Cohen, H. (2001). Gas turbine theory. Pearson Education.

Schwer, D. and Kailasanath, K. (2011). Numerical investigation of the physics of rotatingdetonation-engines. Proceedings of the Combustion Institute, 33(2):2195-2202.

Schwer, D. and Kailasanath, K. (2013). Fluid dynamics of rotating detonation engines with hydrogen and hydrocarbon fuels. Proceedings of the Combustion Institute, 34(2):1991-1998.

Schwer, D. A. and Kailasanath, K. (2012). Feedback into mixture plenums in rotating detonation engines. In 50th AIAA Aerospace Sciences Meeting including the New Horizons Forum and Aerospace Exposition, pages 9-12.

Selle, L., Nicoud, F., and Poinsot, T. (2004). Actual impedance of nonreflecting boundary conditions: Implications for computation of resonators. AIAA journal, 42(5):958-964.

Shao, Y.-T., Liu, M., and Wang, J.-P. (2010). Numerical investigation of rotating detonation engine propulsive performance. Combustion Science and Technology, 182(11-12):1586-1597.

Sharpe, G. J. (2001). Transverse waves in numerical simulations of cellular detonations. Journal of Fluid Mechanics, 447:31-51.

Shepherd, J. (2009). Detonation in gases. Proceedings of the Combustion Institute, 32(1):83-98.

Smith, G. P., Golden, D. M., Frenklach, M., Moriarty, N. W., Eiteneer, B., Goldenberg, M., Bowman, C. T., Hanson, R. K., Song, S., Gardiner Jr, W. C., Vitali, V. L., and Zhiwei, Q. (1999). Gri-mech 3.0.

Sod, G. A. (1978). A survey of several finite difference methods for systems of nonlinear hyperbolic conservation laws. Journal of computational physics, 27(1):1-31.

Sutton, G. P. and Biblarz, O. (2010). Rocket propulsion elements. John Wiley \& Sons.

Swiderski, K. (2013). Numerical Modellingof the Rotating Detonation Combustion Chamber. $\mathrm{PhD}$ thesis, Warsaw University of Technology.

Torregrosa, A., Fajardo, P., Gil, A., and Navarro, R. (2012). Development of non-reflecting boundary condition for application in $3 \mathrm{~d}$ computational fluid dynamics codes. Engineering Applications of Computational Fluid Mechanics, 6(3):447-460. 
Towery, C. A., Smith, K. M., Shrestha, P., Hamlington, P. E., and Van Schoor, M. (2014). Examination of turbulent flow effects in rotating detonation engines. AIAA.

Tsuboi, N., Eto, K., and Hayashi, A. (2007). Detailed structure of spinning detonation in a circular tube. Combustion and flame, 149(1):144-161.

Voitsekhovsky, B. (1960). Stazionarnaia detonatsia (maintained detonation). In Doklady Akad. Nauk SSSR, volume 129, page 1254.

Vutthivithayarak, R., Braun, E., and Lu, F. (2012). On thermodynamic cycles for detonation engines. In 28th International Symposium on Shock Waves, pages 287-292. Springer.

Weiss, J. M. (1999). Calculation of reacting flowfields stiff chemical kinetics. AIAA.

Yi, T.-H., Turangan, C., Lou, J., Wolanski, P., and Kindracki, J. (2009). A three-dimensional numerical study of rotational detonation in an annular chamber. AIAA Paper, 634:2009.

Zhdan, S., Bykovskii, F., and Vedernikov, E. (2007). Mathematical modeling of a rotating detonation wave in a hydrogen-oxygen mixture. Combustion, explosion, and shock waves, 43(4):449-459.

Zhdan, S., Mardashev, A., and Mitrofanov, V. (1990). Calculation of the flow of spin detonation in an annular chamber. Combustion, Explosion, and Shock Waves, 26(2):210-214. 